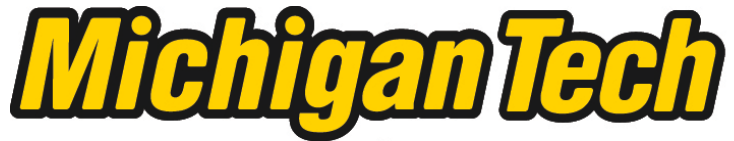 \\ Michigan Technological University Create the Future Digital Commons @ Michigan Tech
}

Dissertations, Master's Theses and Master's Reports - Open

Dissertations, Master's Theses and Master's

Reports

2005

Reconstruction of anisoplanatic adaptive optics images

Mathieu Aubailly

Michigan Technological University

Follow this and additional works at: https://digitalcommons.mtu.edu/etds

Part of the Electrical and Computer Engineering Commons

Copyright 2005 Mathieu Aubailly

\section{Recommended Citation}

Aubailly, Mathieu, "Reconstruction of anisoplanatic adaptive optics images", Dissertation, Michigan Technological University, 2005.

https://doi.org/10.37099/mtu.dc.etds/68

Follow this and additional works at: https://digitalcommons.mtu.edu/etds

Part of the Electrical and Computer Engineering Commons 
Michigan Technological University

Houghton, MI

\title{
Reconstruction of Anisoplanatic Adaptive Optics Images
}

\author{
A dissertation submitted in partial fulfillment of \\ the requirements for the degree of \\ Doctor of PhiLOSOPhY \\ (Electrical Engineering)
}

by

Mathieu Aubailly

2005 
(C) Copyright by

Mathieu Aubailly

2005 
The dissertation of Mathieu Aubailly is approved.

Michael C. Roggemann

Timothy J. Schulz

Jeffrey B. Burl

Alex Kostinski

Michael C. Roggemann, Committee Chair

Michigan Technological University, Houghton, MI

2005

ii 
To my grandparents

Pierre and Yvette, 


\section{TABle of Contents}

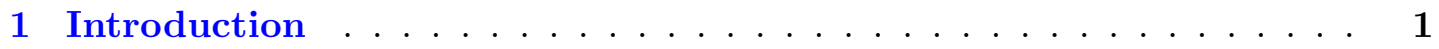

1.1 Astronomical observations . . . . . . . . . . . . . . . . . 1

1.2 Adaptive optics . . . . . . . . . . . . . . . . . . . . 2

1.3 Anisoplanatism . . . . . . . . . . . . . . . . . . . . . 3

1.4 Problem . . . . . . . . . . . . . . . . . . . 4

1.5 Approach . . . . . . . . . . . . . . . . . 4

1.6 Summary of key results $\ldots \ldots \ldots \ldots \ldots$

1.7 Organization of this dissertation $\ldots \ldots \ldots \ldots$

2 Background ...................... 7

2.1 Introduction . . . . . . . . . . . . . . . . . 7

2.2 Fourier Optics. . . . . . . . . . . . . . . . . . 7

2.2 .1 Scalar diffraction theory . . . . . . . . . . . . . . 9

2.2 .2 Helmoltz equation . . . . . . . . . . . . . . . . . . 10

2.2.3 The Rayleigh-Sommerfeld formulation of diffraction . . . . . . . 11

2.2 .4 The Fresnel diffraction . . . . . . . . . . . . . . . . . . . 13

2.2.5 The Fraunhofer diffraction . . . . . . . . . . . . . . 15

2.2 .6 Lenses . . . . . . . . . . . . . . . . . . . . . . . . 16

2.3 Angular spectrum propagator . . . . . . . . . . . . . . . 16

2.3 .1 Definition . . . . . . . . . . . . . . . . 16

2.3.2 Angular spectrum propagation . . . . . . . . . . . . . . 18 
2.4 Statistical Optics . . . . . . . . . . . . . . . . . . . 19

2.4 .1 Introduction . . . . . . . . . . . . . . . . 19

2.4.2 Statistical tools . . . . . . . . . . . . . . . . . 19

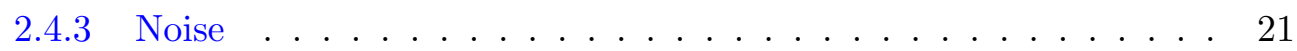

2.4 .4 Photon noise . . . . . . . . . . . . . . . . . 21

2.4.5 Readout noise . . . . . . . . . . . . . . . . . . 22

2.4 .6 Signal-to-noise ratio $\ldots \ldots \ldots \ldots . \ldots \ldots$

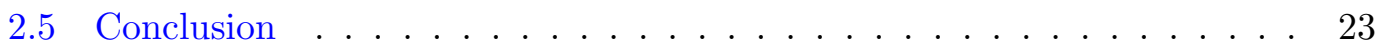

3 Imaging Through Turbulence . . . . . . . . . . . . . . . . . . 24

3.1 Introduction . . . . . . . . . . . . . . . . . . . . . 24

3.2 Historical background . . . . . . . . . . . . . . . . . . . 24

3.3 The index of refraction . . . . . . . . . . . . . . 25

3.4 The turbulence profile . . . . . . . . . . . . . . . . . 27

3.5 The Fried parameter . . . . . . . . . . . . . . . . . . . . . . 29

3.6 Wave propagation in the atmosphere . . . . . . . . . . . . . . 29

3.6.1 Atmospheric model . . . . . . . . . . . . . . . . . . . . . 30

3.6.2 Propagation model . . . . . . . . . . . . . . . . . . . 32

3.7 Simulating turbulence effects on imaging systems . . . . . . . . . . . 33

3.8 Conclusion . . . . . . . . . . . . . . . . . . . . . 34

4 Adaptive Optics . . . . . . . . . . . . . . 36

4.1 Introduction . . . . . . . . . . . . . . . 36

4.2 Approach . . . . . . . . . . . . . . . . . . 36 
4.3 Adaptive optics components . . . . . . . . . . . . . . . . . . 37

4.3 .1 Wavefront sensor . . . . . . . . . . . . . . . . . 37

4.3 .2 Wavefront compensation . . . . . . . . . . . . . . 41

4.3 .3 Control ........................... 45

4.4 Performance measures . . . . . . . . . . . . . . . . . . 48

4.5 Factors limiting performance . . . . . . . . . . . . . . . . 49

4.5 .1 WFS light level . . . . . . . . . . . . . . . . . . 49

4.5 .2 Anisoplanatism . . . . . . . . . . . . . . . . 50

4.5.3 Technical limitations . . . . . . . . . . . . . . . . . 52

4.6 Conclusion . . . . . . . . . . . . . . . . . . 52

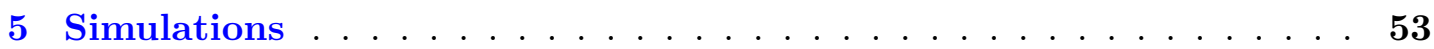

5.1 Introduction . . . . . . . . . . . . . . . . . . 53

5.2 Simulations . . . . . . . . . . . . . . . . . . . 54

5.2 .1 Monte Carlo simulations . . . . . . . . . . . . . . . . . . 54

5.2 .2 Array size . . . . . . . . . . . . . . . . . . 54

5.2 .3 Phase screen generation . . . . . . . . . . . . . . . 55

5.3 Simulation parameters . . . . . . . . . . . . . . . . . . . 59

5.3 .1 Propagation parameters . . . . . . . . . . . . . . . . 59

5.3 .2 Telescope ........................... 59

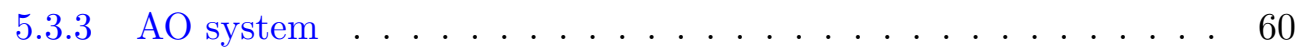

5.4 Sampling requirements . . . . . . . . . . . . . . . . . 60

5.4 .1 Resolution limit . . . . . . . . . . . . . . . . . 61 
5.4 .2 Angular spectrum propagator . . . . . . . . . . . . 62

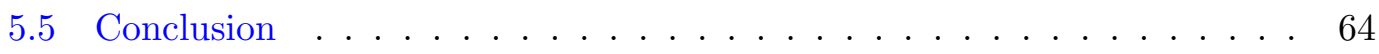

6 Prediction of the off-axis point spread function . . . . . . . 65

6.1 Introduction . . . . . . . . . . . . . . . . . . 65

6.2 Approach . . . . . . . . . . . . . . . . 6 66

6.3 Simulation of the off-axis PSF . . . . . . . . . . . . . . 68

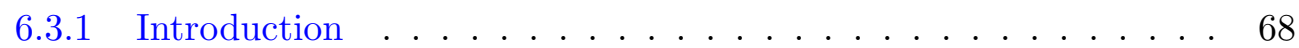

6.3.2 Influence of the field angle on the PSF . . . . . . . . . . 68

6.3.3 Influence of the Fried parameter $r_{0}$ on the PSF $\ldots \ldots \ldots 9$

6.4 Geometrical properties of the PSF . . . . . . . . . . . . . 69

6.4 .1 Introduction . . . . . . . . . . . . . . . . 69

6.4.2 Rotation property . . . . . . . . . . . . . . . 72

6.4.3 Symmetry property . . . . . . . . . . . . . . . 73

6.5 Parameterized model of the PSF . . . . . . . . . . . . . . 74

6.5.1 Model ......................... 74

6.5.2 Model for the PSF cross-section . . . . . . . . . . . . . . 76

6.5.3 Mathematical functions . . . . . . . . . . . . . . . 76

6.5.4 Comparison of the models for the cross-sections . . . . . . . . . 78

6.6 Fitting the model of the PSF to the simulated PSF . . . . . . . . . . 82

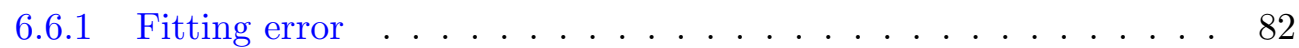

6.6.2 Influence of the field angle on the parameters of the PSF model . 83

6.6.3 Influence of $r_{0}$ on the parameters of the PSF model $\ldots \ldots 84$ 
6.6.4 Influence of the wavefront sensor noise on the parameters of the PSF model . . . . . . . . . . . . . . . . . 85

6.7 PSF prediction by interpolation . . . . . . . . . . . 87

6.7.1 Interpolation in term of the field angle $\ldots \ldots \ldots$. . . . 87

6.8 Results. . . . . . . . . . . . . . . . . . 87

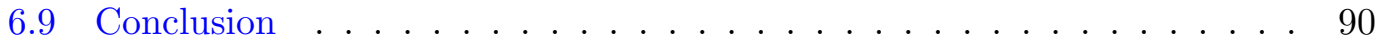

7 Reconstruction of anisoplanatic adaptive optics images . . . . . . 91

7.1 Introduction . . . . . . . . . . . . . . . . . . . . 91

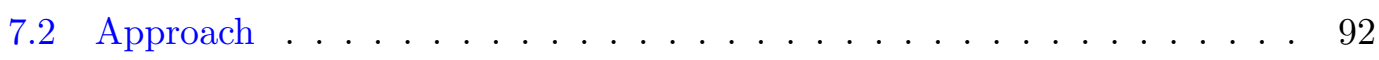

7.3 Imaging model $\ldots \ldots \ldots$. . . . . . . . . . . . . . . . . . 92

7.3.1 Anisoplanatic conditions . . . . . . . . . . . . . . . . 92

$7.3 .2 \quad$ Isoplanatic conditions . . . . . . . . . . . . . . . . 94

7.3 .3 Noise . . . . . . . . . . . . . . . . . . . . 94

7.3.4 Imaging model for a $\theta_{0}$-sized block . . . . . . . . . . . . 95

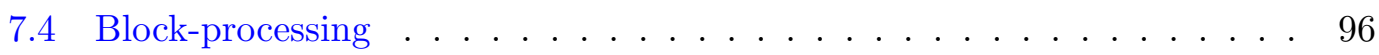

7.5 Image reconstruction . . . . . . . . . . . . . . . . 97

7.5 .1 Introduction . . . . . . . . . . . . . . . . 97

7.5 .2 Inverse problem . . . . . . . . . . . . . . . . 97

7.5.3 Method 1: Tikhonov regularization . . . . . . . . . . . 97

7.5.4 Method 2: Expectation maximization (EM) algorithm . . . . . . 98

7.6 Results. . . . . . . . . . . . . . . . . . . 99

7.6 .1 Introduction $\ldots \ldots \ldots \ldots \ldots$ 
7.6 .2 Error metric . . . . . . . . . . . . . . . . 101

7.6.3 Results for the Tikhonov regularization . . . . . . . . . . . 101

7.6.4 Results for the EM algorithm . . . . . . . . . . . . 102

7.6 .5 Influence of the SNR . . . . . . . . . . . . . . 105

7.6.6 Comparison space-invariant/space-varying PSF deconvolution . . 105

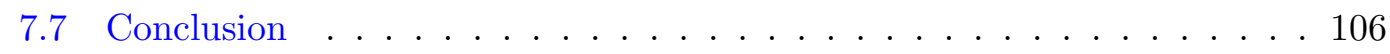

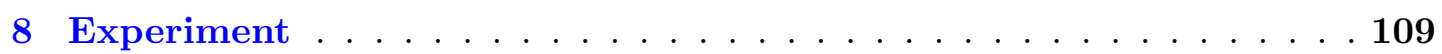

8.1 Introduction . . . . . . . . . . . . . . . . . . . . . . 109

8.2 Data . . . . . . . . . . . . . . . . . . . . . 109

8.3 Data pre-processing . . . . . . . . . . . . . . . . . . 110

8.4 Observation of anisoplanatic effects on images . . . . . . . . . . . . . . 114

8.4 .1 Width of the PSF . . . . . . . . . . . . . . . 114

8.4 .2 Encircled energy . . . . . . . . . . . . . . . . . . . 114

8.4 .3 Peak value of the PSF . . . . . . . . . . . . . 116

8.4 .4 Problem . . . . . . . . . . . . . . . . . . 117

8.5 Deconvolution results . . . . . . . . . . . . . . . . 118

8.6 Conclusion . . . . . . . . . . . . . . . . . . . . . . . 119

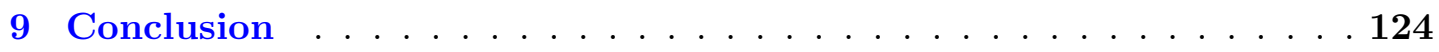

9.1 Summary of results . . . . . . . . . . . . . . . . . . . 124

9.2 Future work . . . . . . . . . . . . . . . . . . 126

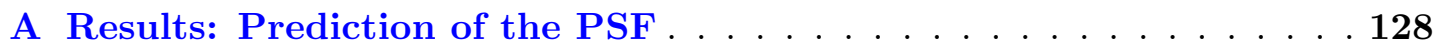


B Results: Reconstruction of anisoplanatic adaptive optics images . . 149

References . . . . . . . . . . . . . . . . . . . . . 180 


\section{List OF FiguRES}

2.1 Diffraction effect on the image of a point source . . . . . . . . 8

2.2 Diffraction geometry . . . . . . . . . . . . . . . . . . 12

2.3 Wave vector . . . . . . . . . . . . . . . . 17

3.1 Turbulence profiles . . . . . . . . . . . . . . . . 28

3.2 Image of a point source through turbulence . . . . . . . . . . . 35

4.1 Organization of an adaptive optics system . . . . . . . . . . . . 38

4.2 Hartmann wavefront sensor . . . . . . . . . . . . . . . . . 39

4.3 Tip-tilt mirror . . . . . . . . . . . . . . . . . 43

4.4 Deformable mirror . . . . . . . . . . . . . . . . . 45

4.5 Anisoplanatism . . . . . . . . . . . . . . . . . . 5 50

5.1 Example of phase screen with the Kolmogorov spatial power spectrum . 59

5.2 Intensity image in the telescope pupil plane . . . . . . . . . . . 62

5.3 Block-diagram of the simulation. . . . . . . . . . . 63

6.1 Block-diagram of the PSF prediction method . . . . . . . . . . 67

6.2 LE AO-corrected PSF's computed for nine values of the field angle. . . . 70

6.3 LE AO-corrected PSF's computed for different $r_{0} \ldots \ldots \ldots 71$

6.4 Rotation property of the PSF . . . . . . . . . . . . . 72

6.5 Symmetry property of the PSF . . . . . . . . . . . 73

6.6 Cross-sections of the PSF . . . . . . . . . . . . . . . 74 


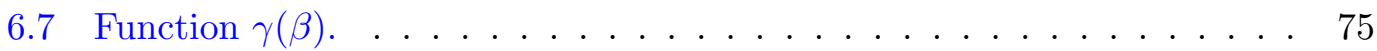

6.8 Relative MS error $\epsilon_{M O D E L}$ vs. order $N \ldots \ldots \ldots \ldots . \ldots \ldots$

6.9 Fitting of the cross-section with different mathematical functions. . . . . 81

6.10 Coefficients $a_{i}$ 's and $b_{i}$ 's vs. $r_{0}$ for $\theta=0 \mu \mathrm{rad} \ldots \ldots . \ldots 86$

6.11 Coefficients $a_{i}$ 's and $b_{i}$ 's vs. $\bar{K}$ for $\theta=0 \mu \mathrm{rad} . \ldots \ldots . \ldots 88$

6.12 Fitting error $\epsilon_{P R E D I C T}$ vs. field angle. . . . . . . . . . . . . 89

7.1 Block diagram of the block-processing method. . . . . . . . . . . . . 93

7.2 Block deconvolution of overlapping blocks. . . . . . . . . . . . . . . 96

7.3 Simulated images for different $r_{0}$ 's. . . . . . . . . . . . 100

7.4 Reconstructed images using the Tikhonov regularization with the predicted PSF and the on-axis PSF for SNR $=10000$ and $r_{0}=15 \mathrm{~cm} . \ldots 103$

7.5 Reconstructed images using the EM algorithm with the predicted PSF and the on-axis PSF for SNR $=10000$ and $r_{0}=15 \mathrm{~cm} . \ldots \ldots 104$

8.1 Examples of raw data. . . . . . . . . . . . . . . . . . . 111

8.2 Pre-processed images of binary stars. . . . . . . . . . . . . . . . 113

8.3 Cross-sections of different binary stars. . . . . . . . . . . . . . 115

8.4 Measured and reconstructed image for binary star STF 738 AB. . . . . 120

8.5 Measured and reconstructed image for binary star STF 611. . . . . . . 121

8.6 Cross-sections of the measured and reconstructed stars: STF 738 AB

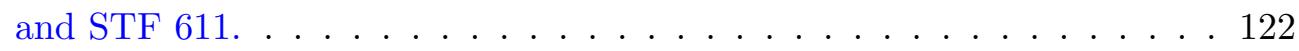

1.1 Fitting error $\epsilon_{\text {FITTING }}$ vs. field angle. . . . . . . . . . . . . . 129

1.2 Parameterized and simulated PSF for different $\theta$, and for $r_{0}=25 \mathrm{~cm} . \quad .130$ 
1.3 Parameterized and simulated PSF for different $\theta$, and for $r_{0}=20 \mathrm{~cm}$. . 131

1.4 Parameterized and simulated PSF for different $\theta$, and for $r_{0}=15 \mathrm{~cm} . \quad \cdot 132$

1.5 Parameterized and simulated PSF for different $\theta$, and for $r_{0}=10 \mathrm{~cm} . \quad .133$

1.6 Coefficients $a_{i}$ 's and $b_{i}$ 's vs. field angle for $r_{0}=25 \mathrm{~cm} \ldots \ldots 134$

1.7 Coefficients $a_{i}$ 's and $b_{i}$ 's vs. field angle for $r_{0}=20 \mathrm{~cm} \ldots \ldots \ldots$

1.8 Coefficients $a_{i}$ 's and $b_{i}$ 's vs. field angle for $r_{0}=15 \mathrm{~cm} \ldots \ldots 136$

1.9 Coefficients $a_{i}$ 's and $b_{i}$ 's vs. field angle for $r_{0}=10 \mathrm{~cm} \ldots \ldots 137$

1.10 Elongation factor $\chi(\theta)$ vs. $\theta . \ldots \ldots \ldots \ldots 138$

1.11 Coefficients $a_{i}$ 's and $b_{i}$ 's vs. $r_{0}$ for $\theta=16 \mu \mathrm{rad} . \ldots \ldots 139$

1.12 Coefficients $a_{i}$ 's and $b_{i}$ 's vs. $r_{0}$ for $\theta=32 \mu \mathrm{rad} \ldots \ldots \ldots . \ldots 140$

1.13 Coefficients $a_{i}$ 's and $b_{i}$ 's vs. $\bar{K}$ for $\theta=16 \mu \mathrm{rad} . \ldots \ldots 141$

1.14 Coefficients $a_{i}$ 's and $b_{i}$ 's vs. $\bar{K}$ for $\theta=32 \mu \mathrm{rad} . \ldots \ldots . . \ldots . . . . .142$

1.15 Predicted and simulated PSF for different $\theta$, and for $r_{0}=25 \mathrm{~cm} . \ldots 143$

1.16 Predicted and simulated PSF for different $\theta$, and for $r_{0}=20 \mathrm{~cm} . \ldots 144$

1.17 Predicted and simulated PSF for different $\theta$, and for $r_{0}=15 \mathrm{~cm} . \ldots 145$

1.18 Predicted and simulated PSF for different $\theta$, and for $r_{0}=10 \mathrm{~cm} . \ldots 146$

1.19 Cross-section of the simulated and predicted PSF, for $r_{0}=25 \mathrm{~cm} . \quad \ldots 147$

1.20 Cross-section of the simulated and predicted PSF, for $r_{0}=25 \mathrm{~cm} . \quad \ldots 148$

2.1 Reconstruction error $\epsilon_{R E C O N S}$ vs. coefficient $\alpha$ for $r_{0}=25 \mathrm{~cm}$ and $20 \mathrm{~cm} .150$

2.2 Reconstruction error $\epsilon_{R E C O N S}$ vs. coefficient $\alpha$ for $r_{0}=15 \mathrm{~cm}$ and $10 \mathrm{~cm} .151$

2.3 Reconstruction error $\epsilon_{R E C O N S}$ vs. iterations for $r_{0}=25 \mathrm{~cm}$ and $20 \mathrm{~cm} .152$

2.4 Reconstruction error $\epsilon_{R E C O N S}$ vs. iterations for $r_{0}=15 \mathrm{~cm}$ and $10 \mathrm{~cm} .153$ 
2.5 Reconstruction error $\epsilon_{R E C O N S}$ vs. SNR for the Tikhonov regularization. 154

2.6 Reconstruction error $\epsilon_{R E C O N S}$ vs. SNR for the EM algorithm. . . . . 155

2.7 Reconstructed images using the Tikhonov regularization with the predicted PSF and the on-axis PSF for SNR $=10000$ and $r_{0}=25 \mathrm{~cm} . \ldots 156$

2.8 Reconstructed images using the Tikhonov regularization with the predicted PSF and the on-axis PSF for SNR $=1$ and $r_{0}=25 \mathrm{~cm} . \ldots 157$

2.9 Reconstructed images using the Tikhonov regularization with the predicted PSF and the on-axis PSF for SNR $=0.5$ and $r_{0}=25 \mathrm{~cm} . \ldots 158$

2.10 Reconstructed images using the Tikhonov regularization with the predicted PSF and the on-axis PSF for SNR $=10000$ and $r_{0}=20 \mathrm{~cm} . . .159$

2.11 Reconstructed images using the Tikhonov regularization with the predicted PSF and the on-axis PSF for SNR $=1$ and $r_{0}=20 \mathrm{~cm} . \ldots 160$

2.12 Reconstructed images using the Tikhonov regularization with the predicted PSF and the on-axis PSF for SNR $=0.5$ and $r_{0}=20 \mathrm{~cm} . \quad \ldots 161$

2.13 Reconstructed images using the Tikhonov regularization with the predicted PSF and the on-axis PSF for SNR $=10000$ and $r_{0}=15 \mathrm{~cm} . . .162$

2.14 Reconstructed images using the Tikhonov regularization with the predicted PSF and the on-axis PSF for SNR $=1$ and $r_{0}=15 \mathrm{~cm} . \ldots 163$

2.15 Reconstructed images using the Tikhonov regularization with the predicted PSF and the on-axis PSF for SNR $=0.5$ and $r_{0}=15 \mathrm{~cm} . \quad \ldots 164$

2.16 Reconstructed images using the Tikhonov regularization with the predicted PSF and the on-axis PSF for SNR $=10000$ and $r_{0}=10 \mathrm{~cm} . . .165$

2.17 Reconstructed images using the Tikhonov regularization with the predicted PSF and the on-axis PSF for SNR $=1$ and $r_{0}=10 \mathrm{~cm} . \ldots 166$ 
2.18 Reconstructed images using the Tikhonov regularization with the predicted PSF and the on-axis PSF for SNR $=0.5$ and $r_{0}=10 \mathrm{~cm} . \quad \ldots 167$

2.19 Reconstructed images using the EM algorithm with the predicted PSF and the on-axis PSF for SNR $=10000$ and $r_{0}=25 \mathrm{~cm} . \ldots \ldots 8$

2.20 Reconstructed images using the EM algorithm with the predicted PSF and the on-axis PSF for SNR $=1$ and $r_{0}=25 \mathrm{~cm} \ldots \ldots 169$

2.21 Reconstructed images using the EM algorithm with the predicted PSF and the on-axis PSF for SNR $=0.5$ and $r_{0}=25 \mathrm{~cm} \ldots \ldots 170$

2.22 Reconstructed images using the EM algorithm with the predicted PSF and the on-axis PSF for SNR $=10000$ and $r_{0}=20 \mathrm{~cm} . \ldots \ldots 171$

2.23 Reconstructed images using the EM algorithm with the predicted PSF and the on-axis PSF for SNR $=1$ and $r_{0}=20 \mathrm{~cm} . \ldots \ldots 172$

2.24 Reconstructed images using the EM algorithm with the predicted PSF and the on-axis PSF for SNR $=0.5$ and $r_{0}=20 \mathrm{~cm} \ldots \ldots 173$

2.25 Reconstructed images using the EM algorithm with the predicted PSF and the on-axis PSF for SNR $=10000$ and $r_{0}=15 \mathrm{~cm} . \ldots \ldots 174$

2.26 Reconstructed images using the EM algorithm with the predicted PSF and the on-axis PSF for SNR $=1$ and $r_{0}=15 \mathrm{~cm} \ldots \ldots 175$

2.27 Reconstructed images using the EM algorithm with the predicted PSF and the on-axis PSF for SNR $=0.5$ and $r_{0}=15 \mathrm{~cm} \ldots \ldots 176$

2.28 Reconstructed images using the EM algorithm with the predicted PSF and the on-axis PSF for SNR $=10000$ and $r_{0}=10 \mathrm{~cm} . \ldots 177$

2.29 Reconstructed images using the EM algorithm with the predicted PSF and the on-axis PSF for SNR $=1$ and $r_{0}=10 \mathrm{~cm} \ldots \ldots 178$ 
2.30 Reconstructed images using the EM algorithm with the predicted PSF and the on-axis PSF for SNR $=0.5$ and $r_{0}=10 \mathrm{~cm} \ldots \ldots 179$ 


\section{LIST OF TABLES}

3.1 Weights $W_{i}$ 's and altitudes $z_{i}$ 's of each layer for different turbulent pro-

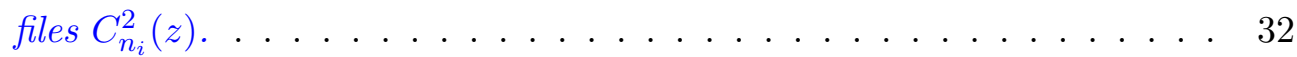

3.2 Weights $W_{i}$ 's and altitudes $z_{i}$ 's of each layer for different turbulent profiles $C_{n_{i}}^{2}(z)$ in the case of uniform thickness layers. . . . . . . . . . . 32

4.1 Zernike polynomials. . . . . . . . . . . . . . . . 42

5.1 Propagation parameters. . . . . . . . . . . . . . . . . 60

5.2 Telescope parameters. . . . . . . . . . . . . . . . . . 61

$5.3 \quad$ AO system parameters. . . . . . . . . . . . . . . . . . . 61

6.1 First seven Chebyshev polynomials of the first kind. . . . . . . . . . 78

6.2 Relative $M S$ error (\%) for fitting the on-axis PSF $(\theta=0$ rrad). . . . . 80

6.3 Relative $M S$ error (\%) for fitting an off-axis PSF, at $\theta=32$ rrad. . . . 80

7.1 Improvement factor $\xi$ for $S N R=10^{4} \ldots \ldots \ldots \ldots$. . . . . . 108

7.2 Improvement factor $\xi$ for $S N R=1 \ldots \ldots \ldots$. . . . . . . 108

7.3 Improvement factor $\xi$ for $S N R=0.5 \ldots \ldots \ldots \ldots$

8.1 Binary stars measured at the AEOS. . . . . . . . . . . . . . . . 112

8.2 Computed and measured intensities for four binary stars. . . . . . . . 116

8.3 Computed and measured normalized peak values of binary stars. . . . . 117

8.4 Reconstruction errors. . . . . . . . . . . . . . . . . . . . . . . . 119 


\section{ACKNOWLEDGMENTS}

I want to thank:

Dr. Mike Roggemann for being my advisor during this research project, as well as my comittee members Dr. Timothy Schulz, Dr. Jeffrey Burl, and Dr. Alex Kostinski. My family, in particular my grandparents Pierre and Yvette, my parents Jean-Pierre and Monique, and of course my sister Anne-Lise for their support, understanding and care.

My friends in the US as well as in Europe for their presence. No need to mention names, they know.

The local music scene of Houghton, which makes me enjoy this area with a different lifetyle: the Upper Peninsula of Michigan. 


\section{CHAPTER 1}

\section{Introduction}

\subsection{Astronomical observations}

Dating back to antiquity, astronomy is possibly the oldest of the natural sciences. Originally, astronomy consisted of observing and predicting the position of visible celestial objects. The Greek philosophers invented several models to explain the motions of stars, planets, the moon and the sun. The renaissance of astronomy occurred in 1543 when Nicolaus Copernicus proposed a heliocentric model for celestial objects. In the $17^{\text {th }}$ century, Galileo was among the first to use a telescope to observe the sky. It is in 1610 that he discovered the four moons of Jupiter.

Over time, astronomical telescopes evolved considerably. At first telescopes were small (of the order of few centimeters) and gradually grew toward very large telescopes: up to 10 meters diameter. Different projects of very large telescopes are on their way like the Giant Magellan Telescope with an aperture of 21.4 meters. The underlying motivation for building larger telescopes is the quest for image quality. The size of the aperture of the telescope affects the observation results in two different manners. First, a large aperture allows collection of more light, and therefore detection of fainter and more distant objects. Second, the limiting angular resolution of the telescope increases with its aperture size, providing sharper images. If telescopes are used in a perfect vacuum, the resolution is directly proportional to the inverse of the telescope 
diameter. The angular resolution is then only limited by the diffraction of light, a phenomenon also known as the diffraction limit. However, in the case of ground-based telescopes, the light coming from a star or another celestial object goes through the Earth's atmosphere. The atmosphere causes random spatial and temporal wavefront perturbations of the light, and blurs images produced by ground-based telescopes. This explains, in part, why we launch telescopes into space, like the Hubble Space Telescope. As an example, for very large ground-based telescopes (more than 8 meters diameter) the resolution is reduced by a factor of 50 to 100 [1]. Many techniques had been developed over time to improve the resolution of ground-based telescopes [2]. The next section presents a relatively new technique, known as adaptive optics [3].

\subsection{Adaptive optics}

Adaptive optics (AO) is a technology used in large ground-based telescopes to reduce the effect of atmospheric turbulence $[2,4,5,6,7,8]$. Adaptive optics works by measuring the distortion of light and compensating for it in real-time. The wavefront distortion is corrected using deformable mirrors. The turbulence-induced distortion varies on the timescale of milliseconds. Therefore a fast computer is used to reshape the surface of the deformable mirror accordingly. The incoming light falls on the deformable mirror and bounces back with its atmospheric-induced distortion removed, or at least partially removed. As a consequence, the image formed appears sharper.

The atmospheric distortion is measured by a wavefront sensor. The most used one is the Shack-Hartmann wavefront sensor. Because objects of interest are often too faint to be used as the reference for the Shack-Hartmann wavefront sensor, a nearby bright star is necessary. This brighter star is called the guide star. The need for a reference guide star means that adaptive optics system cannot be used in every direction of 
observation, but only where there is a guide star with sufficient luminosity nearby the object. Usually, a guide star must be brighter than magnitude of 11 or 12 . In the 1980's a solution was proposed to overcome this limitation: the laser guide star (LGS) [9]. The idea is to shoot a laser beam into the atmosphere in the direction of interest. The light scattering from atmospheric constituents forms a spot of light. This spot of light can be used as a reference for the adaptive optics system. Since the object and the reference guide star are nearby (in term of angular separation), the light of the object passes through approximately the same atmospheric turbulence than the light of the reference star. Therefore, its image is also corrected, but less accurately.

\subsection{Anisoplanatism}

Anisoplanatism results from the fact that the light coming from different directions in the scene does not go through the same atmospheric turbulence [10]. Therefore, the turbulence-induced aberration is different for different directions. The isoplanatic angle is defined as the maximum angular separation between the object we look at and the guide star for which the turbulence-induced aberration is approximately constant.

Anisoplanatism affects the performance of adaptive optics systems [11, 12]. The larger the angular separation between the object and the reference beacon, the more blurred the image is. Adaptive optics systems are considered to achieve diffractionlimited correction of the wavefront in the isoplanatic angle only. The isoplanatic angle, which depends on the turbulence profile is denoted $\theta_{0}$ and is typically of the order of $10 \mu \mathrm{rad}$ and is centered on the reference guide star. 


\subsection{Problem}

Due to anisoplanatism, the correctable field of view (FOV) of adaptive systems is limited in size to approximately the isoplanatic angle $\theta_{0}$, which fundamentally limits the AO performance since many astronomical objects, as well as satellites, exceed the dimension of the isoplanatic patch. Additionally, AO systems can only compensate in one direction. In this dissertation we seek a post-processing approach to overcome these problems.

\subsection{Approach}

In this dissertation, I propose an image post-processing technique to overcome some of the effects of anisoplanatism in large FOV adaptive optics images. The reconstruction method is organized in two steps:

1. The first step of the technique consists of predicting the space-varying point spread function (PSF) as a function of the field angle. A wave optics simulation is used to compute the PSF at certain field angles, denoted $\theta_{\text {sim }}$. Those simulated PSF's are fit with a parameterized model for the PSF. Finally, by interpolating the coefficients of the model, we predict the PSF for all angles between the $\theta_{\text {sim }}$ 's. The knowledge of the PSF is essential in image restoration.

2. The second step consists of reconstructing large FOV images using the PSF predicted in Step 1. A block-processing method is used: the image is split into approximately $\theta_{0}$-sized patches over which the PSF is approximately constant. Each block is then deconvolved with widely used methods for image recovery: matrix inversion with Tikhonov regularization, and the expectation maximization (EM) algorithm. The deconvolved blocks are then reassembled to form the 
restored image. An error metric is then defined in order to quantify the improvement from the original image to the reconstructed image.

\subsection{Summary of key results}

In this dissertation, a model for the long exposure (LE) AO-corrected PSF is introduced, and a method for the prediction of the PSF as a function of the field angle is presented. The method is based on the interpolation of the parameters of the model of the PSF. The mean square (MS) error between the predicted PSF and the simulated PSF varies between $0.9 \%$ and $2.7 \%$ depending on the field angle and the seeing conditions.

In order to validate the PSF prediction method, we reconstruct anisoplanatic adaptive optics images using the predicted off-axis PSF. The MS error between the reconstructed image and the object varies from $4 \%$ to $45 \%$ depending on the seeing conditions and the signal-to-noise ratio (SNR). The reconstructed images are compared to the reconstructed images using the on-axis PSF. The reconstruction method using the predicted PSF shows an improvement of the MS error of $7.2 \%$ to $84.8 \%$.

\subsection{Organization of this dissertation}

This dissertation is organized as follows. Chapter 2 is a background chapter and introduces the reader to the theoretical basis of wave optics. Chapter 3 presents the problem of imaging through turbulence. In chapter 4, adaptive optics systems are studied in detail. Chapter 5 describes the wave propagation simulations performed for the prediction of the PSF. In chapter 6, the method for prediction of the PSF as a function of the field angle is introduced, and results are given. In chapter 7, the knowledge of the 
PSF as a function of the field angle is used to reconstruct anisoplanatic AO-corrected images. The performance of the image restoration method is estimated. Chapter 8 summarizes the experiment, and gives the results obtained. Finally, chapter 9 is a conclusion. 


\section{CHAPTER 2}

\section{Background}

\subsection{Introduction}

In this chapter, we present the theoretical basis of wave optics. In section 2.2 , after a qualitative introduction to the field of Fourier optics, we present the phenomenon of diffraction. Different diffraction formulas are established. The first formula is called the Rayleigh-Sommerfeld formulation and is the most general form. Then, some approximations are used to give the Fresnel and the Fraunhofer diffraction formulas. In section 2.4 an overview of statistical optics is given to introduce the statistical tools used in atmospheric optics. Finally, photon noise and readout noise are discussed in detail, and the concept of the signal-to-noise ratio is presented.

\subsection{Fourier Optics}

Diffraction can be defined [13] as any deviation of light rays from rectilinear path that cannot be interpreted as reflection or refraction. Fourier optics is used to explain the physical effects arising from the wave nature of light. In chapter 3 , we discuss limitations of imaging systems when propagating light through a turbulent media like atmosphere. However, other non-turbulent-induced limitations also occur. For example, diffraction is a limiting factor for imaging systems even when the light propagates in free space. 
Diffraction can easily be demonstrated with simple experiments. For example, if we examine the intensity image of a point source through a circular aperture of diameter $D$ when propagating into free space over a distance $Z$. While geometrical optics predicts the image of a point source to be a point, the diffraction-limited image (see Figure 2.1) is a blurry disk. This pattern is explained by diffraction theory and is referred to as the Airy disk. We can notice the side lobes in the intensity pattern, which is a characteristic feature of the Airy disk. Since the Airy disk is broader than a focused point, diffraction limits the resolution of imaging systems. To improve the resolution of imaging systems, the size of the Airy disk should be reduced. This can be done either by decreasing the wavelength $\lambda$, either by increasing the size of the aperture $D$. This last point explains why larger and larger telescopes are always being designed. The minimum resolvable angle of an imaging system is on the order of $\lambda / D$. After describing diffraction qualitatively, we give mathematical expressions of diffraction in the next sections.

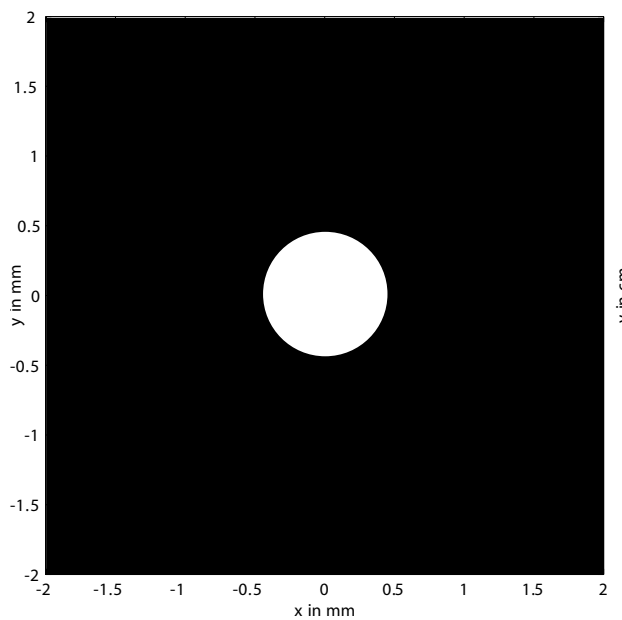

(a)

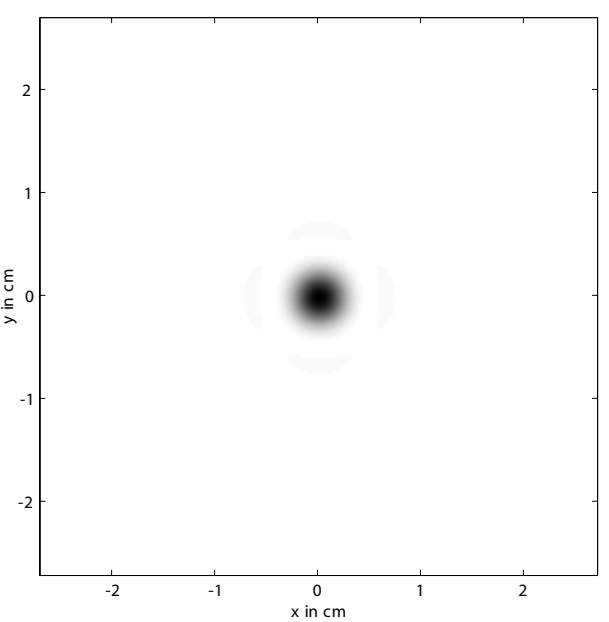

(b)

Figure 2.1: (a) Circular aperture of diameter $D=0.85 \mathrm{~mm}$. (b) Intensity image of a point source through the circular aperture of size $D$ when propagating in free space. The propagation distance is $Z=10 \mathrm{~m}$, and the wavelength is $\lambda=850 \mathrm{~nm}$. 


\subsubsection{Scalar diffraction theory}

\subsubsection{Vector theory to scalar theory}

The foundation of the diffraction theory lies in Maxwell's equations for a source-free region [14]:

$$
\begin{aligned}
\nabla \times \vec{E} & =-\mu \frac{\partial \vec{H}}{\partial t}, \\
\nabla \times \vec{H} & =-\epsilon \frac{\partial \vec{E}}{\partial t}, \\
\nabla \cdot \epsilon \vec{E} & =0, \\
\nabla \cdot \mu \vec{H} & =0,
\end{aligned}
$$

where $\vec{E}$ is the electric field and its orthogonal components are $\left(E_{x}, E_{y}, E_{z}\right)$. The orthogonal components of the magnetic field $\vec{H}$ are $\left(H_{x}, H_{y}, H_{z}\right)$. The wave propagates in a medium of permeability $\mu$ and permittivity $\epsilon . \nabla$ denotes the Laplacian operator. Applying the Laplacian operator to both sides of Maxwell's equation 2.2 yields

$$
\nabla \times(\nabla \times \vec{E})=\nabla(\nabla \cdot \vec{E})-\nabla^{2} \vec{E}
$$

Furthermore, we assume that the medium is linear, isotropic, homogeneous, nondispersive, and non-magnetic $\left(\mu=\mu_{0}\right)$. Substituting Maxwell's equations 2.2 and 2.4 into Eq. 2.5, we obtain the wave equation for $\vec{E}$ :

$$
\nabla^{2} \vec{E}-\epsilon \mu_{0} \frac{\partial^{2} \vec{E}}{\partial t^{2}}=0
$$

We now define the index of refraction $n$ by

$$
n=\sqrt{\frac{\epsilon}{\epsilon_{0}}}
$$

and the velocity of propagation in vacuum $c$ by

$$
c=\frac{1}{\sqrt{\mu_{0} \epsilon_{0}}},
$$


which leads us to a more common expression of the wave equation:

$$
\nabla^{2} \vec{E}-\frac{n^{2}}{c^{2}} \frac{\partial^{2} \vec{E}}{\partial t^{2}}=0
$$

Similar calculations prove that the magnetic field also respects Eq. 2.9:

$$
\nabla^{2} \vec{H}-\frac{n^{2}}{c^{2}} \frac{\partial^{2} \vec{H}}{\partial t^{2}}=0
$$

Therefore, for both fields $\vec{E}$ and $\vec{H}$, each of their orthogonal component obeys

$$
\nabla^{2} u_{k}-\frac{n^{2}}{c^{2}} \frac{\partial^{2} u_{k}}{\partial t^{2}}=0
$$

where $u_{k}$ corresponds to any of the components of $\vec{E}$ or $\vec{H}$. It should be noted that Eq. 2.11 is a scalar equation. However, this is true only because we made assumptions earlier in this section. We can note that we also assume all propagation distances to be much larger than $\lambda$, and all apertures to have dimensions much larger than $\lambda$.

\subsubsection{Helmoltz equation}

In the previous section, we introduced the time and space dependent variable $u_{k}$, which can be expressed, in the case of a monochromatic wave, by:

$$
u_{k}(x, y, z, t)=A(x, y, z) \cos (2 \pi \nu t+\phi(x, y, z))
$$

where $\nu$ is the optical frequency, $A(x, y, z)$ is the amplitude of the wave, and $\phi(x, y, z)$ is the phase at the spatial position $(x, y, z)$. Denoting the real part of a complex number by $\Re\{\cdot\}$, Eq. 2.12 can be written:

$$
u_{k}(x, y, z, t)=\Re\left\{U_{k}(x, y, z) \exp (-2 \pi \nu t)\right\},
$$

where

$$
U_{k}(x, y, z)=A(x, y, z) \exp \{-j \phi(x, y, z)\}
$$


By multiplying both sides of the wave equation (Eq. 2.11) by $\exp (+2 \pi \nu t)$, the time dependence disappears and we obtain the following equation for $U_{k}(x, y, z)$ :

$$
\left(\nabla^{2}+k^{2}\right) U_{k}=0
$$

where $k$ is the wave number defined by

$$
k=2 \pi n \frac{\nu}{c}=\frac{2 \pi}{\lambda}
$$

Eq. 2.15 is called the Helmoltz equation.

\subsubsection{The Rayleigh-Sommerfeld formulation of diffraction}

The Rayleigh-Sommerfeld formulation of diffraction is the most general expression of diffraction. The diffraction formula is derived from the Helmoltz equation (Eq. 2.15), and physically and mathematically reasonable boundary conditions imply that we are working in the conditions above:

1. The aperture and the objects we consider are large compared to the optical wavelength $\lambda$.

2. The distance $z$ between the aperture plan and the observation plane is much larger than $\lambda$.

Figure 2.2 shows the geometry. The field in the observation plane $\Sigma_{1}$ is to be calculated from the knowledge of the field incident onto the aperture plane $\Sigma_{0}$. The RayleighSommerfeld formulation of diffraction is [14]:

$$
u_{1}\left(P_{1}\right)=\frac{1}{j \lambda} \int_{\Sigma_{0}} u_{o}\left(P_{0}\right) \frac{\exp \left(j k r_{01}\right)}{r_{01}} \cos (\theta) d P_{0}
$$

where $P_{0}$ is point in the aperture plane, $P_{1}$ is a point in the observation plane, $r_{01}$ is defined by $r_{01}=\left|\vec{r}_{01}\right|, \lambda$ is the wavelength, $k$ is the wave number defined in Eq. 2.16, and 


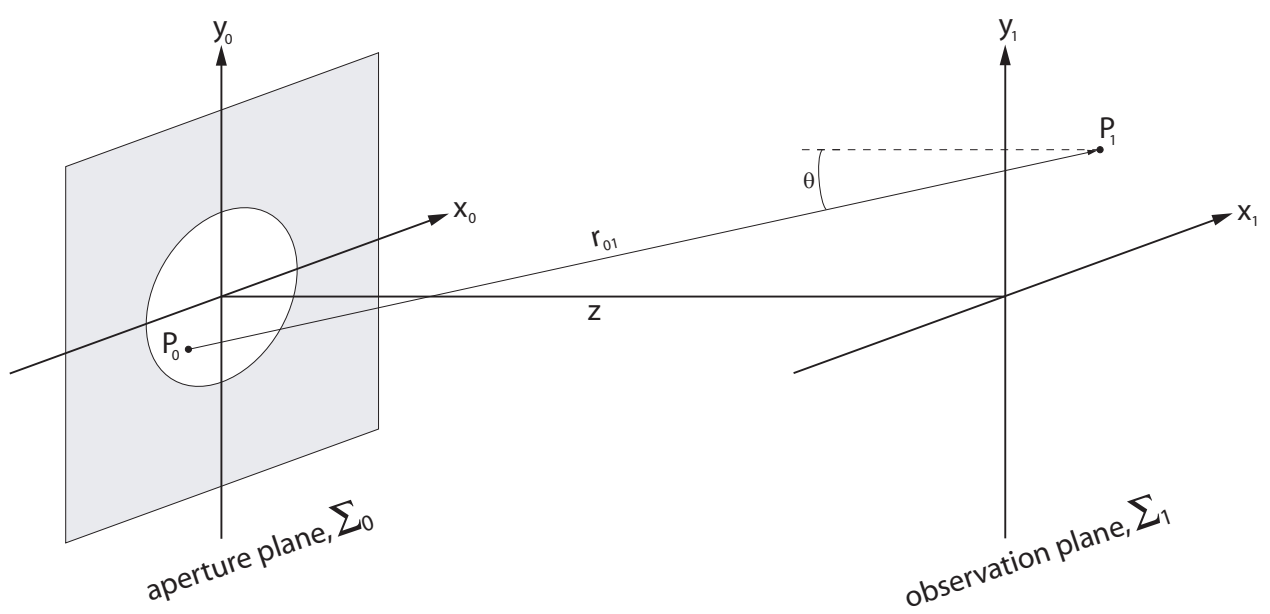

Figure 2.2: Diffraction geometry.

the term $\cos (\theta)$ is called the obliquity factor. The integral is over the entire aperture plane $\Sigma_{0}$ defined by

$$
\Sigma_{0}= \begin{cases}1 & \text { inside the aperture } \\ 0 & \text { outside the aperture }\end{cases}
$$

\section{Linear system approach}

We can look at the propagation of light in free space from a linear system point of view. The field in the aperture plan can be regarded as the input of the system and the field in the observation plan as the output. In this interpretation the field $u_{1}\left(P_{1}\right)$ is given by

$$
u_{1}\left(P_{1}\right)=\int_{\Sigma_{0}} u_{o}\left(P_{0}\right) h\left(P_{1}, P_{0}\right) d P_{0}
$$

where $h\left(P_{1}, P_{0}\right)$ is the impulse response of the system expressed by

$$
h\left(P_{1}, P_{0}\right)=\frac{1}{j \lambda} \frac{\exp \left(j k r_{01}\right)}{r_{01}} \cos (\theta) .
$$

The impulse response $h\left(P_{1}, P_{0}\right)$ is shift invariant:

$$
\begin{aligned}
h\left(P_{1}, P_{0}\right) & =h\left(P_{1}-P_{0}\right), \\
& =h\left(\overrightarrow{x_{1}}-\overrightarrow{x_{0}}\right),
\end{aligned}
$$


where $\overrightarrow{x_{0}}$ and $\overrightarrow{x_{1}}$ are the position vectors of $P_{0}$ and $P_{1}$. Therefore, the field in the image plane $u_{1}\left(\overrightarrow{x_{1}}\right)$ can be written as the convolution of the input field $u_{0}\left(\overrightarrow{x_{0}}\right)$ and the impulse response $h\left(\overrightarrow{x_{1}}-\overrightarrow{x_{0}}\right)$ :

$$
\begin{aligned}
u_{1}\left(\overrightarrow{x_{1}}\right) & =\int_{\Sigma_{0}} u_{o}\left(\overrightarrow{x_{0}}\right) h\left(\overrightarrow{x_{1}}-\overrightarrow{x_{0}}\right) d \overrightarrow{x_{0}}, \\
& =u_{o} * h,
\end{aligned}
$$

where $*$ denotes the convolution operation. Fourier transforming both sides of Eq. 2.24 yields

$$
U_{1}(\vec{f})=U_{0}(\vec{f}) H(\vec{f})
$$

where $U_{1}(\vec{f}), U_{0}(\vec{f})$, and $H(\vec{f})$ are the two dimensional Fourier transforms of $u_{1}\left(\overrightarrow{x_{1}}\right)$, $u_{0}\left(\overrightarrow{x_{0}}\right)$, and $h\left(\overrightarrow{x_{1}}-\overrightarrow{x_{0}}\right)$, respectively. $H(\vec{f})$ is the transfer function of the system and is defined by [14]

$$
H(\vec{f})= \begin{cases}\exp \left\{j 2 \pi \sqrt{\frac{1}{\lambda^{2}}-|\vec{f}|^{2}}\right\} & \text { for }|\vec{f}|<\frac{1}{\lambda} \\ 0 & \text { elsewhere. }\end{cases}
$$

In the next two section, we study two special cases of the Rayleigh-Sommerfeld diffraction formula: the Fresnel diffraction formula (section 2.2.4) and the Fraunhofer diffraction formula (section 2.2.5).

\subsubsection{The Fresnel diffraction}

The Fresnel diffraction formula is an approximation of the Rayleigh-Sommerfeld diffraction formula and gives accurate results for points near the optical axis. 


\subsubsection{Fresnel approximation}

In the Rayleigh-Sommerfeld formulation of diffraction (Eq. 2.17), the term $r_{01}$ appears and can be written exactly as

$$
r_{01}=\sqrt{\left(x_{1}-x_{0}\right)^{2}+\left(y_{1}-y_{0}\right)^{2}+z^{2}}
$$

where $\overrightarrow{x_{0}}=\left(x_{0}, y_{0}\right)$ and $\overrightarrow{x_{1}}=\left(x_{1}, y_{1}\right)$. In addition to the assumptions previously made (section 2.2.3), we assume that the propagation distance $z$ is much larger than the transverse coordinates $\sqrt{\left(x_{1}-x_{0}\right)^{2}+\left(y_{1}-y_{0}\right)^{2}}$. The Fresnel approximation is therefore:

1. The distance $r_{01}$ between $P_{0}$ and $P_{1}$ is approximately equal to $z$, the distance between the aperture plane and the observation plane. Thus, the obliquity factor $\cos (\theta)$ equals one. This is called the paraxial condition.

2. Using a binomial expansion (first order only) of $r_{01}$ given by Eq. 2.27, $r_{01}$ is approximated by

$$
r_{01} \approx z+\frac{\left(x_{1}-x_{0}\right)^{2}+\left(y_{1}-y_{0}\right)^{2}}{2 z} .
$$

\subsubsection{Fresnel diffraction formula}

Substituting Eq. 2.28 in Eq. 2.20, the free space propagation impulse response becomes

$$
h_{\text {Fresnel }}\left(\overrightarrow{x_{1}}\right)=\frac{1}{j \lambda} \frac{\exp (j k z)}{z} \exp \left\{j \frac{k}{2 z}\left\{\left(x_{1}-x_{0}\right)^{2}+\left(y_{1}-y_{0}\right)^{2}\right\}\right\}
$$

The Fresnel diffraction formula is then [14]:

$$
\begin{aligned}
u_{1}\left(\overrightarrow{x_{1}}\right)= & \frac{1}{j \lambda} \exp \left\{j \frac{k}{2 z}\left|\overrightarrow{x_{1}}\right|^{2}\right\} \\
& \times \int_{\Sigma_{0}} u_{o}\left(\overrightarrow{x_{0}}\right) \exp \left\{j \frac{k}{2 z}\left|\overrightarrow{x_{0}}\right|^{2}\right\} \exp \left\{j \frac{2 \pi}{\lambda z} \overrightarrow{x_{0}} \cdot \overrightarrow{x_{1}}\right\} d \overrightarrow{x_{0}} .
\end{aligned}
$$


In Eq. 2.30, the integral can be recognized as a Fourier transform. The formula is then simplified to

$$
u_{1}\left(\overrightarrow{x_{1}}\right)=\frac{1}{j \lambda} \exp \left\{j \frac{k}{2 z}\left|\overrightarrow{x_{1}}\right|^{2}\right\} \mathcal{F}\left\{u_{o}\left(\overrightarrow{x_{0}}\right) \exp \left\{j \frac{k}{2 z}\left|\overrightarrow{x_{0}}\right|^{2}\right\}\right\}_{\vec{f}=\overrightarrow{x_{1}} / \lambda z},
$$

where $\mathcal{F}\{\cdot\}$ denotes the Fourier transform operation, and $\vec{f}=\overrightarrow{x_{1}} / \lambda z$ means that the Fourier transform is evaluated for this frequency value. From Eq. 2.29, we obtain the transfer function of the system for the Fresnel approximation [15]:

$$
H_{\text {Fresnel }}(\vec{f})=\left\{\begin{array}{ll}
\exp \{j k z\} \exp \left\{-j \pi \lambda z|\vec{f}|^{2}\right\} & \text { for }|\vec{f}|<\frac{1}{\lambda} \\
0 & \text { elsewhere }
\end{array} .\right.
$$

\subsubsection{The Fraunhofer diffraction}

\subsubsection{Fraunhofer approximation}

The Fraunhofer approximation consists of considering the distance of propagation $z$ much larger than the quadratic term $\frac{k}{2}\left|\overrightarrow{x_{0}}\right|^{2}$ in Eq. 2.31:

$$
z \gg \frac{k}{2}\left|\overrightarrow{x_{0}}\right|^{2} \text {. }
$$

This approximation is more restrictive than the Fresnel approximation and is called the far field approximation.

\subsubsection{Fraunhofer diffraction formula}

Assuming the Fraunhofer approximation is true, the term $\exp \left\{j \frac{k}{2 z}\left|\overrightarrow{x_{0}}\right|^{2}\right\}$ in Eq. 2.31 can be neglected and the diffraction formula becomes

$$
u_{1}\left(\overrightarrow{x_{1}}\right)=\frac{1}{j \lambda} \exp \left\{j \frac{k}{2 z}\left|\overrightarrow{x_{1}}\right|^{2}\right\} \mathcal{F}\left\{u_{o}\left(\overrightarrow{x_{1}}\right)\right\}_{\vec{f}=\overrightarrow{x_{1}} / \lambda z} .
$$

We can notice the simplicity of the Eq. 2.34. The field in the observation plane is obtained by multiplying the Fourier transform of the incident field by a quadratic term depending on $z$. 


\subsubsection{Lenses}

The transmittance function of a lens is given by [15]

$$
t_{\text {lens }}(\vec{x})=\exp \left\{-j \frac{k}{2 f}|\vec{x}|^{2}\right\}
$$

where $k$ is the wave number and $f$ the focal length of the lens. The relation between the transmitted field $u_{\text {trans }}(\vec{x})$ and the incident field $u_{\text {incid }}(\vec{x})$ is then:

$$
u_{\text {trans }}(\vec{x})=u_{\text {incid }}(\vec{x}) t_{\text {lens }}(\vec{x})
$$

\subsection{Angular spectrum propagator}

\subsubsection{Definition}

The key idea of the angular spectrum propagator is to decompose the field to propagate in a sum of plane waves using Fourier transforms (FT) [14]. Every wave plane propagates in a different direction, and has a unique amplitude and phase. The resulting field in the target plane is the superposition of the propagated plane waves, each of which is affected by a different phase shift.

Lets consider the incident wave $u(x, y, 0)$ in the aperture plane $z=0$. The complex field propagates along the $z$-axis. We want to calculate the field $u(x, y, z)$ for $z>0$. First, the two dimensional Fourier transform of $u(x, y, 0)$ is given by

$$
A\left(f_{x}, f_{y}, 0\right)=\iint_{-\infty}^{\infty} u(x, y, 0) \exp \left\{-j 2 \pi\left(f_{x} x+f_{y} y\right)\right\} d x d y
$$

where $f_{x}$ and $f_{y}$ are the spatial frequencies. If we write $u(x, y, 0)$ as the inverse FT of $A\left(f_{x}, f_{y}, 0\right)$, we can look at $u(x, y, 0)$ as a weighted sum of complex exponential function:

$$
u(x, y, 0)=\iint_{-\infty}^{\infty} A\left(f_{x}, f_{y}, 0\right) \exp \left\{j 2 \pi\left(f_{x} x+f_{y} y\right)\right\} d f_{x} d f_{y} .
$$


The kernel of this inverse Fourier transform, $\exp \left\{j 2 \pi\left(f_{x} x+f_{y} y\right)\right\}$, can be interpreted as the complex representation of a plane wave. This one can be denoted $P(x, y, z)$ with

$$
\begin{aligned}
P(x, y, z) & =\exp \{j \vec{k} \cdot \vec{r}\} \\
& =\exp \left\{j \frac{2 \pi}{\lambda}(\alpha x+\beta y+\gamma z)\right\},
\end{aligned}
$$

where $\vec{r}=x \vec{a}+y \vec{b}+z \vec{c}$ is the position vector, and $\vec{k}$ is the wave vector defined by $\vec{k}=2 \pi / \lambda(\alpha \vec{a}+\beta \vec{b}+\gamma \vec{c})$. The wave vector gives the direction of propagation. The vectors $\vec{a}, \vec{b}$, and $\vec{c}$ are unit vectors. Since $\alpha^{2}+\beta^{2}+\gamma^{2}=1$, we can regard $P(x, y, z)$ as a plane wave traveling in the direction (See Figure 2.3):

$$
\begin{aligned}
& \theta_{\alpha}=\cos ^{-1}(\alpha)=\cos ^{-1}\left(\lambda f_{x}\right), \\
& \theta_{\beta}=\cos ^{-1}(\beta)=\cos ^{-1}\left(\lambda f_{y}\right), \\
& \theta_{\gamma}=\cos ^{-1}(\gamma)=\cos ^{-1}\left(\sqrt{1-\left(\lambda f_{x}\right)^{2}+\left(\lambda f_{y}\right)^{2}}\right) .
\end{aligned}
$$

Using this notation, the angular spectrum of $u(x, y, 0)$ is defined by

$$
A\left(\frac{\alpha}{\lambda}, \frac{\beta}{\lambda}, 0\right)=\iint_{-\infty}^{\infty} u(x, y, 0) \exp \left\{-j 2 \pi\left(\frac{\alpha}{\lambda} x+\frac{\beta}{\lambda} y\right)\right\} d x d y
$$

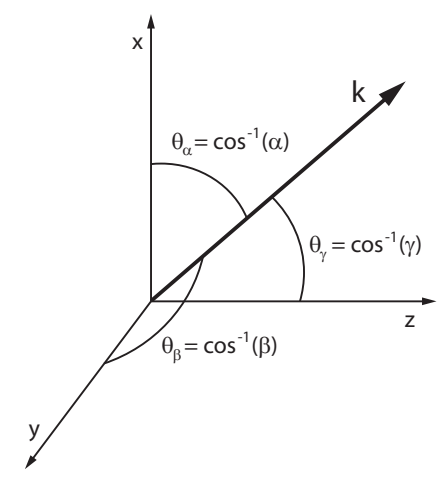

Figure 2.3: Wave vector $\vec{k}$. 


\subsubsection{Angular spectrum propagation}

We now want to know the relation between $A\left(f_{x}, f_{y}, 0\right)$ and the angular spectrum of $u(x, y, z)$ for $z>0$. The angular spectrum of $u(x, y, z), A\left(f_{x}, f_{y}, z\right)$, is defined by

$$
A\left(\frac{\alpha}{\lambda}, \frac{\beta}{\lambda}, z\right)=\iint_{-\infty}^{\infty} u(x, y, z) \exp \left\{-j 2 \pi\left(\frac{\alpha}{\lambda} x+\frac{\beta}{\lambda} y\right)\right\} d x d y
$$

We can easily reverse Eq. 2.43 to obtain $u(x, y, z)$ :

$$
u(x, y, z)=\iint_{-\infty}^{\infty} A\left(\frac{\alpha}{\lambda}, \frac{\beta}{\lambda}, z\right) \exp \left\{j 2 \pi\left(\frac{\alpha}{\lambda} x+\frac{\beta}{\lambda} y\right)\right\} d \frac{\alpha}{\lambda} d \frac{\beta}{\lambda}
$$

The wave that we consider is monochromatic and thus must satisfy the Heltmoltz equation established in Eq. 2.15:

$$
\nabla^{2} u(x, y, z)+k^{2} u(x, y, z)=0
$$

Substituting Eq. 2.44 in Eq. 2.45, we obtain the following differential equation:

$$
\frac{d^{2}}{d z^{2}} A\left(\frac{\alpha}{\lambda}, \frac{\beta}{\lambda}, z\right)+\left(\frac{2 \pi}{\lambda}\right)^{2}\left(1-\alpha^{2}-\beta^{2}\right) A\left(\frac{\alpha}{\lambda}, \frac{\beta}{\lambda}, z\right)=0 .
$$

A solution of Eq. 2.46 is given by [14]

$$
A\left(\frac{\alpha}{\lambda}, \frac{\beta}{\lambda}, z\right)=A\left(\frac{\alpha}{\lambda}, \frac{\beta}{\lambda}, 0\right) \exp \left\{j \frac{2 \pi}{\lambda} \sqrt{1-\alpha^{2}-\beta^{2}} z\right\}
$$

where $\alpha^{2}+\beta^{2}<1$. From Eq. 2.47 we can see that the propagation of a wave corresponds to a phase change of the angular spectrum. The delay introduced for the plane wave component traveling in the direction $\left(\theta_{\alpha}, \theta_{\beta}, \theta_{\gamma}\right)$ is $\Delta \varphi=\frac{2 \pi}{\lambda} \sqrt{1-\alpha^{2}-\beta^{2}} z$. By inverting Eq. 2.47, we finally obtain the expression of $u(x, y, z)$ :

$$
\begin{aligned}
u(x, y, z)= & \iint_{-\infty}^{\infty}\left(\frac{\alpha}{\lambda}, \frac{\beta}{\lambda}, 0\right) \exp \left\{j \frac{2 \pi}{\lambda} \sqrt{1-\alpha^{2}-\beta^{2}} z\right\} \\
& \times \exp \left\{j 2 \pi\left(\frac{\alpha}{\lambda} x+\frac{\beta}{\lambda} y\right)\right\} d \frac{\alpha}{\lambda} d \frac{\beta}{\lambda},
\end{aligned}
$$

for $\alpha^{2}+\beta^{2}<1$. 


\subsection{Statistical Optics}

\subsubsection{Introduction}

The atmosphere is a random medium, therefore a statistical description of the key atmospheric parameters is necessary. When imaging through turbulence, a complete model of optical imaging system should take into account the random noise associated with the light detection process. There are two sources of measurement noise [15]. The first source of noise is due to the random times and locations of photons falling on the light detector. This noise originates from the quantum nature of light and is referred to as photon noise, Poisson noise, or shot noise. The second type of noise originates from the readout electronics of charge-coupled device-based detectors, and is referred to as readout noise.

In this section we give a review of useful statistical tools, and we present into details the photon noise, as well as the readout noise.

\subsubsection{Statistical tools}

Lets consider the random process $X(\vec{x}, t)$, where $t$ is the time and $\vec{x}$ the vector position in space. The statistical average of the random process is defined by

$$
E\{X(\vec{x}, t)\}=\int_{-\infty}^{\infty} X(\vec{x}, t) f_{X}(x) d x
$$

where $f_{X}$ is the probability density function of $X(\vec{x}, t)$. The space-time covariance of $X$ is given by

$$
\operatorname{Cov}_{X}\left(\overrightarrow{x_{1}}, \overrightarrow{x_{2}}, t_{1}, t_{2}\right)=E\left\{\left[X\left(\overrightarrow{x_{1}}, t_{1}\right)-E\left\{X\left(\overrightarrow{x_{1}}, t_{2}\right)\right\}\right]\left[X\left(\overrightarrow{x_{2}}, t_{2}\right)-E\left\{X\left(\overrightarrow{x_{2}}, t_{2}\right)\right\}\right]^{*}\right\}
$$


The correlation function is defined by

$$
\Gamma_{X}\left(\overrightarrow{x_{1}}, t_{1}, \overrightarrow{x_{2}}, t_{2}\right)=E\left\{X\left(\overrightarrow{x_{1}}, t_{1}\right) X^{*}\left(\overrightarrow{x_{2}}, t_{2}\right)\right\}
$$

When a random process is stationary in the wide-sense, its statistics respect certain conditions:

- The expected value $E\{X(\vec{x}, t)\}$ is time-independent: $E\{X(\vec{x}, t)\}=\mu(\vec{x})$,

- The second order moments depend only on the time difference $\tau=t_{1}-t_{2}$ : $\Gamma_{X, t}\left(\overrightarrow{x_{1}}, \overrightarrow{x_{2}}, \tau\right)=E\left\{X\left(\overrightarrow{x_{1}}, t\right) X^{*}\left(\overrightarrow{x_{2}}, t-\tau\right)\right\}$.

When a random process is homogeneous:

- The expected value $E\{X(\vec{x}, t)\}$ is translation invariant: $E\{X(\vec{x}, t)\}=\mu(t)$,

- The second order moments depend only on the translation $\vec{\rho}=\overrightarrow{x_{1}}-\overrightarrow{x_{2}}$ :

$$
\Gamma_{X, s}\left(\vec{\rho}, t_{1}, t_{2}\right)=E\left\{X\left(\vec{x}, t_{1}\right) X^{*}\left(\vec{x}-\vec{\rho}, t_{1}, t_{2}\right)\right\} .
$$

If a random process is ergodic, the expected value equals the time average:

$$
\langle X(\vec{x}, t)\rangle=\frac{1}{T} \int_{T} X(\vec{x}, t) d t,
$$

where $T$ is a time interval. The temporal power spectral density (PSD) given by

$$
\Phi_{t}(\nu)=\int_{-\infty}^{\infty} \Gamma_{X, t}\left(\overrightarrow{x_{1}}, \overrightarrow{x_{2}}, \tau\right) \exp \{-j 2 \pi \nu \tau\} d \tau
$$

and the spatial power spectral density is given by

$$
\Phi_{s}(\vec{\kappa})=\left(\frac{1}{2 \pi}\right)^{3} \iint \Gamma_{X, s}\left(\vec{\rho}, t_{1}, t_{2}\right) \exp \{-j \vec{\kappa} \cdot \vec{\rho}\} d \vec{\rho},
$$

where $\nu$ is the temporal frequency and $\vec{\kappa}$ is the spatial frequency. The temporal and spatial correlation functions are then given by

$$
\Gamma_{X, t}\left(\overrightarrow{x_{1}}, \overrightarrow{x_{2}}, \tau\right)=\int_{-\infty}^{\infty} \Phi_{t}(\nu) \exp \{j 2 \pi \nu \tau\} d \tau
$$


and

$$
\Gamma_{X, s}\left(\vec{\rho}, t_{1}, t_{2}\right)=\iint \Phi_{s}(\vec{\kappa}) \exp \{j \vec{\kappa} \cdot \vec{\rho}\} d \vec{\kappa}
$$

respectively. If a random process is homogeneous and also isotropic, then:

$$
\Gamma_{X, s}\left(\vec{\rho}, t_{1}, t_{2}\right)=\Gamma_{X, s}\left(\rho, t_{1}, t_{2}\right)
$$

where $\rho=|\vec{\rho}|$. In the isotropic case, the spatial power spectrum (Eq. 2.54) becomes

$$
\Phi_{s}(\kappa)=\left(\frac{1}{2 \pi^{2} \kappa}\right) \int_{0}^{\infty} \rho \Gamma_{X, s}\left(\rho, t_{1}, t_{2}\right) \sin \{\kappa \rho\} d \rho,
$$

where $\kappa=|\vec{\kappa}|$. The correlation function becomes

$$
\Gamma_{X, s}\left(\rho, t_{1}, t_{2}\right)=\frac{4 \pi}{\rho} \int_{0}^{\infty} \kappa \Phi_{s}(\kappa) \sin \{\kappa \rho\} d \kappa
$$

\subsubsection{Noise}

The model for a detected image $d(\vec{x})$ is given by

$$
d(\vec{x})=\sum_{i=1}^{M} \delta\left(\vec{x}-\overrightarrow{x_{i}}\right)+\sum_{p=1}^{P} n_{p} \delta\left(\vec{x}-\overrightarrow{x_{p}}\right),
$$

where $\overrightarrow{x_{i}}$ is the location of the $i^{\text {th }}$ photonevent on the light detector and $M$ the total number of photonevents forming the image. The image is composed of $P$ pixels and $n_{p}$ denotes the random variable corresponding to the readout noise at the $p^{\text {th }}$ pixel. In section 2.4.4 and section 2.4.5, the photon noise and the readout noise are respectively studied.

\subsubsection{Photon noise}

The first source of noise that we study is the photon noise. The physical origin of the photon noise is attributed to the quantum nature of light. The photon noise is due to the random arrival times and locations of photoevents on the light detector. It is 
also referred to as shot noise, and Poisson noise. Let us consider the random variable $K$, corresponding to the number of photonevents falling on the light detector. In the literature, the random variable $K$ is often assumed to obey the Poisson distribution [14]:

$$
f_{K}(k, \lambda)=\frac{e^{-\lambda} \lambda^{k}}{k !}
$$

where $\lambda$ is a parameter called the rate function. Photon noise is therefore signaldependent.

\subsubsection{Readout noise}

The second source of noise we consider is the readout noise. Readout noise originates from the readout electronics of light detectors and detector material $[16,17]$. The readout noise differs from the photon noise in the sense that it is signal-independent. It is an additive noise. A commonly used model for the distribution of the readout noise is a zero-mean Gaussian distribution.

\subsubsection{Signal-to-noise ratio}

When imaging through turbulence, we need a statistical tool to measure the effect of noise on detected images. A commonly used metric is the signal-to-noise ratio (SNR), which evaluates the effect of noise on the image. Several definition of the SNR of an image exist. However, the SNR is often defined as

$$
S N R=\frac{\sigma_{s}}{\sigma_{n}},
$$

where $\sigma_{s}$ and $\sigma_{n}$ denote respectively the standard deviation of the detected image $d(\vec{x})$ and the noise. 


\subsection{Conclusion}

In the first part of this chapter, the basis of wave optics had been given into details. The field of Fourier optics had been presented, and the phenomenon of diffraction introduced. Diffraction formulas are given for the general case, as well as for some approximations: the Fresnel approximation for points near the optical axis, and the Fraunhofer approximation for far field propagation. The second part of the chapter deals with statistical optics. Statistical tools, necessary to provide key parameters of the atmosphere and describe the propagation of light through a random medium, are given into details. Models were given for the two sources of noise interfering the light detection process: the photon noise and the readout noise. The concept of signal-tonoise ratio was finally presented. 


\section{CHAPTER 3}

\section{Imaging Through Turbulence}

\subsection{Introduction}

Atmospheric turbulence has an effect on every imaging system which must form images through the atmosphere [15]. The properties of the light going through a long atmospheric path are affected in both time and space. A consequence of this is that the atmosphere limits the average resolution of ground-based telescopes. In groundbased imaging, the challenge consists of compensating the effect of the atmosphere on images. We begin this chapter by giving a historical background on imaging through turbulence. Then, we explain how the index of refraction plays an important role in wave propagation through the atmosphere. A model for the atmosphere is introduced, as well as a wave propagation model. Finally the effects of turbulence on imaging systems are described.

\subsection{Historical background}

The effects of turbulence on imaging systems were recognized by Isaac Newton. Newton noticed that ground-based telescopes were unable to reach diffraction limited performance. He noticed that the point spread function of a telescope looking through turbulence was broader than the one expected if looking through a vacuum. This 
phenomenon was identified as a consequence of atmospheric turbulence. Easily observable, the twinkling of stars was well known too. Newton also understood that placing observatories atop of high mountain would decrease the effects of atmospheric turbulence [15]:

"The only Remedy is a most serene and quiet Air, such as may perhaps be found on the top of the highest Moutains above the grosser Clouds."

However, it is interesting to note that Isaac Newton wrote in 1730 in Opticks that he saw no solution to the problem of atmospheric turbulence limiting the performance of telescopes [18]. In Newton's days, the effect of turbulence could be partially understood, but not corrected. Babcock was a precursor in the field and presented in 1953 one of the first use of adaptive optics [19]. He proposed to compensate for atmospheric distortions that affected telescope images by using a deformable optical element. He also proposed the use of a wavefront sensor. The concept of adaptive optics is studied in chapter 4 .

\subsection{The index of refraction}

Inhomogeneities of air density in the atmosphere cause the index of refraction to fluctuate. The mechanism behind it can be explained with fluid dynamics concepts and the Kolmogorov theory of turbulence [15, 20, 21, 22]. The unpredictable nature of air motion makes the index of refraction random.

We can model the index of refraction $n(\vec{r}, t)$ as the sum of its mean, $n_{0}$, and its fluctuation, $n_{1}(\vec{r}, t)$ :

$$
n(\vec{r}, t)=n_{0}+n_{1}(\vec{r}, t),
$$

where $\vec{r}$ is the three dimensional vector position, and $t$ the time. For air, the mean 
index of refraction is $n_{0}=1$. The fluctuation term $n_{1}(\vec{r}, t)$ can be expressed [23]:

$$
n_{1}=n-1=\frac{77.6 P}{T} \times 10^{-6},
$$

where $T$ is the air temperature (in Kelvins), and $P$ the air pressure (in millibars). We consider locally homogeneous and isotropic pockets of air, also called turbulent eddies [15]. Let $\Phi_{n}(\vec{k})$ denote the spatial power spectral density (PSD) of $n_{1}(\vec{r})$. The variable $\vec{\kappa}$ is called the spatial wavenumber vector and its components are $\left(\kappa_{x}, \kappa_{y}, \kappa_{z}\right)$. $\Phi_{n}(\vec{k})$ is a measurement of the density of turbulent eddies of size $l_{x}, l_{y}$, and $l_{z}$, with $l_{x}=2 \pi / \kappa_{x}, l_{y}=2 \pi / \kappa_{y}$, and $l_{z}=2 \pi / \kappa_{z}$. We now want to have an expression for $\Phi_{n}(\vec{\kappa})$. It is assumed homogeneity and isotropy properties to apply. $\Phi_{n}(\vec{\kappa})$ is then a function of the scalar wavenumber:

$$
\kappa=\sqrt{\kappa^{2}+\kappa^{2}+\kappa^{2}} .
$$

The Kolmogorov theory [23] gives an expression of $\Phi_{n}(\kappa)$ for $\kappa \in\left[2 \pi / L_{0}, 2 \pi / l_{0}\right]$. $L_{o}$ and $l_{0}$ are parameters of the atmosphere referred to as the outer scale and the inner scale, respectively. They represent the characteristic dimension of the largest and the smallest turbulent eddies for which the spatial PSD is predicted by the Kolmogorov theory, referred to as the inertial subrange [15]. Within the inertial subrange the Kolmogorov spectrum is given by

$$
\Phi_{n}^{K}(\vec{\kappa})=0.033 C_{n}^{2}(z) \kappa^{-11 / 3}
$$

where $C_{n}^{2}(z)$ is called the structure constant of the index of refraction fluctuations and has units of $\mathrm{m}^{-2 / 3}$. The parameter $z$ is the distance to the aperture. Since $\Phi_{n}^{K}(\vec{\kappa})$ is not defined for $\kappa=0$, an alternate spectrum expression, called the von Karman spectrum, is sometimes used. The von Karman spectrum is given by

$$
\Phi_{n}^{V}(\vec{\kappa})=\frac{0.033 C_{n}^{2}(z)}{\left(\kappa^{2}+\kappa_{0}^{2}\right)^{11 / 6}} \exp \left\{-\frac{\kappa^{2}}{\kappa_{m}^{2}}\right\},
$$

where $\kappa_{0}=2 \pi / L_{0}$, and $\kappa_{m}=5.92 / l_{0}$. With this expression of the spectrum, $\Phi_{n}^{V}(\vec{\kappa})$ has a finite value for $\vec{\kappa}=0$. 


\subsection{The turbulence profile}

The term $C_{n}^{2}(z)$ characterizes the turbulence strength. The distance to the aperture $z$ depends on the angle of observation with the zenith, denoted $\theta_{z}$. The distance $z$ is related to the altitude $h$ by [15]

$$
z=\frac{h}{\cos \left(\theta_{z}\right)}
$$

Thus we can express $C_{n}^{2}$ as a function of $h$. Numerous models have been developed for $C_{n}^{2}$. In this section, we provide a few of the commonly used ones. The Hufnagel-Valley turbulence profile is given by $[9,24]$

$$
\begin{aligned}
C_{n}^{2}(h)= & 5.94 \times 10^{-53}(v / 27)^{2} h^{10} \exp \{-h / 1000\} \\
& +2.7 \times 10^{-16} \exp \{-h / 1500\}+A \exp \{-h / 100\}
\end{aligned}
$$

where $A$ characterizes the turbulence strength near ground level. Typically, we take $A=1.7 \times 10^{-} 14 \mathrm{~m}^{-2 / 3}$. The parameter $v$ represents the high altitude wind speed, and a commonly used value for $v$ is $v=21 \mathrm{~m} / \mathrm{s}$. Another daytime $C_{n}^{2}(h)$ profile is the Submarine Laser Communication (SLC) profile [9]:

$$
C_{n}^{2}(h)=\left\{\begin{array}{lr}
0 & 0 \mathrm{~m} \quad<h<19 \mathrm{~m} \\
4.008 \times 10^{-13} h^{-1.054} & 19 \mathrm{~m} \quad<h<230 \mathrm{~m} \\
1.300 \times 10^{-15} & 230 \mathrm{~m}<h<850 \mathrm{~m} \\
6.352 \times 10^{-7} h^{-2.966} & 850 \mathrm{~m} \quad<h<7000 \mathrm{~m} \\
6.209 \times 10^{-16} h^{-0.6229} & 7000 \mathrm{~m} \quad<h<20,000 \mathrm{~m}
\end{array}\right.
$$

The most two commonly used nighttime profiles are the modified Hufnagel-Valley profile, given by [9]

$$
\begin{aligned}
C_{n}^{2}(h)= & 8.16 \times 10^{-54} h^{10} \exp \{-h / 1000\} \\
& +3.02 \times 10^{-17} \exp \{-h / 1500\}+1.90 \times 10^{-15} \exp \{-h / 100\},
\end{aligned}
$$


and the Greenwood profile given by [25]

$$
C_{n}^{2}(h)=\left[2.2 \times 10^{-} 13(h+10)^{-1.3}+4.3 \times 10^{-17}\right] \times \exp \{-h / 4000\} .
$$

In this dissertation, we use a turbulence profile corresponding to the island of Maui, HI. The profile is called Maui3 and is defined by

$$
C_{n}^{2}(h)=\left\{\begin{array}{lr}
10^{-9.401-1.5913 h / 1000-0.0606(h / 1000)^{2}} & 0 \mathrm{~m}<h<4,200 \mathrm{~m} \\
10^{-17.1273-0.0332 h / 1000-0.0015(h / 1000)^{2}}+0.9061 & \\
\left.\times \exp \left\{-0.5 \times\left(\frac{15.0866-h / 1000}{5.2977}\right)^{2}\right)\right\} & 4,200 \mathrm{~m} \quad<h<30,000 \mathrm{~m}
\end{array}\right.
$$

Figure 3.1 is plot of the different turbulence profiles $C_{n}^{2}(h)$ as a function of the altitude $h$.

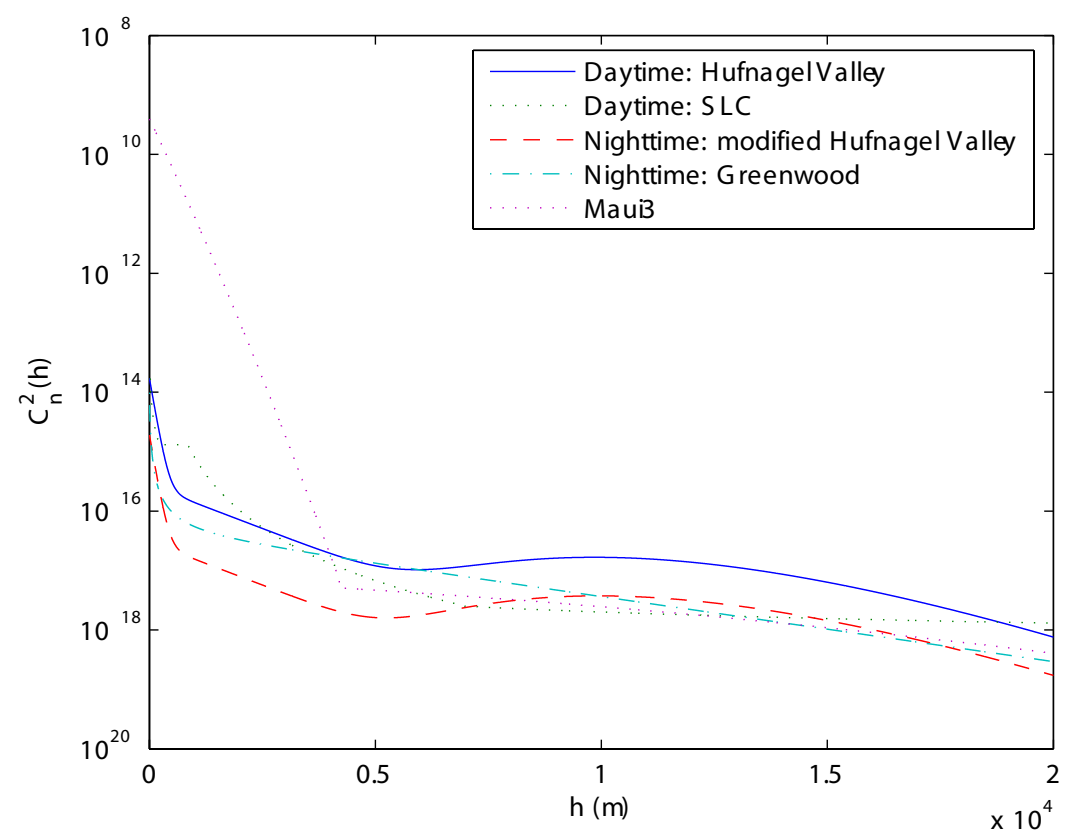

Figure 3.1: Turbulence profiles. 


\subsection{The Fried parameter}

The Fried parameter $r_{0}$ for plane wave propagation through a turbulent region is given by $[26]$

$$
r_{0}=0.185\left[\frac{4 \pi^{2}}{k^{2} \int_{0}^{L} C_{n}^{2}(z)}\right]^{3 / 5},
$$

where $L$ is the propagation distance through the turbulence region and $C_{n}^{2}$ is the continuous profile of the structure constant. The Fried parameter can be interpreted as the aperture size of the imaging system beyond which an increase of the diameter does not result in an increase of the resolution.

\subsection{Wave propagation in the atmosphere}

Maxwell's equations govern wave propagation phenomenon [27]. Electric fields have been showed to have an important role in the propagation of light. Assuming that the propagation medium is linear, isotropic, homogeneous, and non-dispersive, Maxwell's equations yield the wave equation for the electric field $\vec{E}$ given by

$$
\nabla^{2} \vec{E}-\frac{n^{2}}{c^{2}} \frac{\partial^{2} \vec{E}}{\partial t^{2}}=0
$$

where $\nabla^{2}$ is the Laplacian operator, $c$ is the velocity of light in free space (or vacuum), and $n$ is the index of refraction defined by

$$
n=\sqrt{\frac{\epsilon}{\epsilon_{0}}}
$$

where $\epsilon_{0}$ is the vacuum permittivity. We saw in section 3.3 that the index of refraction $n$ fluctuates randomly in time and space. Therefore, Eq. 3.13 can not be solved exactly. However, some solutions based on the Huygens-Fresnel principle had been proposed [28]. Consider the propagation of the field $u_{0}\left(\overrightarrow{r_{0}}\right)$ through a random media. The field in the observation plane is denoted $u_{1}\left(\overrightarrow{r_{1}}\right)$. The vectors $\overrightarrow{r_{0}}$ and $\overrightarrow{r_{1}}$ correspond 
to coordinates respectively in the aperture and the observation plane. The field $u_{1}\left(\overrightarrow{r_{1}}\right)$ is given by

$$
u_{1}\left(\overrightarrow{r_{1}}\right)=\int u_{o}\left(\overrightarrow{r_{0}}\right) h\left(\overrightarrow{r_{1}}, \overrightarrow{r_{0}}\right) d \overrightarrow{r_{0}}
$$

where $h\left(\overrightarrow{r_{1}}, \overrightarrow{r_{0}}\right)$ is the impulse response in the case of a random media, and is given by $[14]$

$$
h\left(\overrightarrow{r_{1}}, \overrightarrow{r_{0}}\right)=\frac{1}{j \lambda} \frac{\exp \left\{j k\left|\overrightarrow{r_{1}}-\overrightarrow{r_{0}}\right|\right\}}{\left|\overrightarrow{r_{1}}-\overrightarrow{r_{0}}\right|} \exp \left\{\varphi\left(\overrightarrow{r_{1}}, \overrightarrow{r_{0}}\right)\right\}
$$

where $\lambda$ is the wavelength, and $\varphi\left(\overrightarrow{r_{1}}, \overrightarrow{r_{0}}\right)$ is a fluctuation term due to the random media. $\varphi\left(\overrightarrow{r_{1}}, \overrightarrow{r_{0}}\right)$ is written

$$
\varphi\left(\overrightarrow{r_{1}}, \overrightarrow{r_{0}}\right)=\exp \left\{\chi\left(\overrightarrow{r_{1}}, \overrightarrow{r_{0}}\right)+j \phi\left(\overrightarrow{r_{1}}, \overrightarrow{r_{0}}\right)\right\}
$$

The term $\chi\left(\overrightarrow{r_{1}}, \overrightarrow{r_{0}}\right)$ is the logarithm of the amplitude fluctuations, and $\phi\left(\overrightarrow{r_{1}}, \overrightarrow{r_{0}}\right)$ is the phase fluctuation. $\phi\left(\overrightarrow{r_{1}}, \overrightarrow{r_{0}}\right)$ accounts for the global phase change between the aperture plane and the target plane. For propagation through a thin layer of atmosphere, the phase change is expressed by

$$
\phi(\vec{r}, h)=k n_{1}(\vec{r}, h) \delta h,
$$

where $k=2 \pi / \lambda$ is the wave number, $n_{1}$ the fluctuation of the index of refraction, and $\delta h$ the thickness of the layer of atmosphere. Propagation through the atmosphere causes phase aberration, however, over long distances, it also causes amplitude aberrations [29, $30]$.

\subsubsection{Atmospheric model}

Since analytical solutions to some atmospheric optics problems are either hard, or in some cases impossible to find, numerical simulations are widely accepted means of modeling performance. Numerical methods require a model for the atmospheric turbulence. A layered model for atmospheric turbulence is described in Ref. [15]. This method is commonly used because it greatly simplifies the calculations, and is easily adapted to 
computer simulations. This model is presented in this section. The main idea is that the atmosphere is seen as pile of horizontal layers. By splitting the atmosphere into $N_{\text {layers }}$ layers, the turbulence profile $C_{n}^{2}(z)$ can be considered to be reasonably constant within each layer. We denote $z_{i}$ the altitude of the $i$ th layer and $\Delta z_{i}$ its thickness. The corresponding structure constant is designated by $C_{n_{i}}^{2}$. The propagation through each layer is computed separately and then recombined to obtain the global effect of turbulence. We can associate a weight $W_{i}$ to each layer such that $\sum_{i}^{N_{\text {layers }}} W_{i}=1$. For each layer, the weight $W_{i}$, the altitude $z_{i}$ and $C_{n_{i}}^{2}$ are computed to respect the following moment equation:

$$
\sum_{i=1}^{N_{\text {layers }}} z_{i}^{m} C_{n_{i}}^{2} \Delta z_{i}=\int_{0}^{L} z^{m} C_{n}^{2}(z) d z,
$$

where $0 \leq m \leq 7$ and $L$ is the propagation distance through the turbulence. The weights $W_{i}$ 's are given by

$$
W_{i}=\frac{C_{n_{i}}^{2} \Delta z_{i}}{\int_{0}^{\infty} C_{n}^{2}(z) d z}
$$

In Ref. [31], Troxel et. al. describe a four layer model for turbulence. However, more accurate wave propagation modelling requires more screens. Troxel et. al. also compute the weights $W_{i}$ 's and altitudes $z_{i}$ 's for different turbulent profiles $C_{n_{i}}^{2}(z)$ (See Table 3.1). We can notice in Table 3.1 that different turbulence profiles can lead to very different weights. Also, the weight $W_{1}$ is always that largest, which means that the lowest layers of the atmosphere are always the most turbulent. Turbulence layers could be chosen to have uniform thickness $\Delta z_{i}=L / N_{\text {layers }}$ for $i=1$ to $N_{\text {layers }}$. If the first layer is assumed to be in pupil plane of the imaging system, the altitude $z_{i}$ of each layer is given by

$$
\begin{aligned}
z_{i} & =(i-1) \frac{L}{N_{\text {layers }}}, \\
& =(i-1) \Delta z_{i} .
\end{aligned}
$$




\begin{tabular}{|r|cccc|}
\hline$C_{n_{i}}^{2}(z)$ & $W_{1}$ & $W_{2}$ & $W_{3}$ & $W_{4}$ \\
& $(200 \mathrm{~m})$ & $(2,000 \mathrm{~m})$ & $(10,000 \mathrm{~m})$ & $(18,000 \mathrm{~m})$ \\
\hline Hufnagel-Valley 21 & 0.8902 & 0.0443 & 0.0591 & 0.0064 \\
Nighttime SLD & 0.4965 & 0.4623 & 0.0299 & 0.0113 \\
Daytime SLD & 0.7397 & 0.2513 & 0.0048 & 0.0042 \\
Greenwood & 0.8615 & 0.0980 & 0.0394 & 0.0011 \\
\hline
\end{tabular}

Table 3.1: Weights $W_{i}$ 's and altitudes $z_{i}$ 's of each layer for different turbulent profiles $C_{n_{i}}^{2}(z)$.

Thus, the integrated structure constant $C_{n_{i}}^{2}(z)$ for each layer is computed by

$$
C_{n_{i}}^{2}=\int_{z_{i}}^{z_{i}+\Delta z_{i}} C_{n}^{2}(z) d z
$$

Finally, the weights are given by Eq. 3.20. Table 3.2 shows the weights of each layer for a model with four layers of uniform thickness. The propagation distance through the turbulence is $L=20,000 \mathrm{~m}$. In this dissertation, we use layers of uniform thickness.

\begin{tabular}{|r|cccc|}
\hline$C_{n_{i}}^{2}(z)$ & $W_{1}$ & $W_{2}$ & $W_{3}$ & $W_{4}$ \\
& $(0 \mathrm{~m})$ & $(5,000 \mathrm{~m})$ & $(10,000 \mathrm{~m})$ & $(15,000 \mathrm{~m})$ \\
\hline Hufnagel-Valley 21 & 0.9364 & 0.0299 & 0.0274 & 0.0063 \\
Nighttime SLD & 0.8843 & 0.0512 & 0.0524 & 0.0121 \\
Daytime SLD & 0.9858 & 0.0069 & 0.0040 & 0.0033 \\
Greenwood & 0.8894 & 0.0815 & 0.0227 & 0.0064 \\
\hline
\end{tabular}

Table 3.2: Weights $W_{i}$ 's and altitudes $z_{i}$ 's of each layer for different turbulent profiles $C_{n_{i}}^{2}(z)$ in the case of uniform thickness layers.

\subsubsection{Propagation model}

For each layer of the atmosphere, the propagation method follows those steps [32]: 
1. The incoming wave $u\left(\overrightarrow{x_{0}}\right)$ falls on a phase screen. This phase screen accounts for the phase aberration occurring during the propagation through the next layer. The method for computing the phase screens is explained in section 5.2.3. The layers are assumed to be thin enough to affect only the phase and not the amplitude of the field. The field resulting field $u^{\prime}\left(\overrightarrow{x_{0}}\right)$ after the phase screen is given by

$$
u^{\prime}\left(\overrightarrow{x_{0}}\right)=u\left(\overrightarrow{x_{0}}\right) \exp \left\{j \Phi\left(\overrightarrow{x_{0}}\right)\right\}
$$

where $\overrightarrow{x_{0}}$ is the vector position in the phase screen plane, and $\Phi\left(\overrightarrow{x_{0}}\right)$ the random phase aberration.

2. The field $u^{\prime}(\vec{x})$ is now propagated through free space or vacuum using the angular spectrum method studied in section 2.3. The angular spectrum propagation is performed using fast Fourier transforms (FFT). The propagated field $u^{\prime \prime}\left(\overrightarrow{x_{1}}\right)$ is then given by

$$
u^{\prime \prime}\left(\overrightarrow{x_{1}}\right)=\mathcal{F}^{-1}\left\{\mathcal{F}\left\{u^{\prime}\left(\overrightarrow{x_{0}}\right)\right\} H\left(\overrightarrow{f_{x}}\right)\right\}
$$

where $H\left(\overrightarrow{f_{x}}\right)$ is the free space transfer function, and $\overrightarrow{x_{1}}$ the vector position in the target plane.

3. If the propagation through the considered volume of turbulence is not finished, go to step 1 for propagation through the next layer.

\subsection{Simulating turbulence effects on imaging sys- tems}

In this section we deal with the effects of turbulence on imaging systems. Every groundbased imaging system is affected by the atmosphere. For example, the smallest resolvable angle for a ground-based astronomical telescope is significantly larger (up to 
$50-100$ times for large telescope) than the angle predicted by the theory of diffraction. To illustrate the effect of the atmosphere, we propagate the light coming from a point source through the atmosphere using a simulation and observe the image formed. The propagation distance is $15 \mathrm{~km}$, and the Fried parameter $r_{0}$ is $15 \mathrm{~cm}$. The pupil of the imaging system has a diameter of $D=3.67 \mathrm{~m}$, and the wavelength used is $\lambda=850 \mathrm{~nm}$. According to the Sparrow resolution criterion (See chapter 2), the minimum resolvable angle is $\lambda / D \approx 0.23 \mu \mathrm{rad}$. Figure 3.2 shows the short exposure and long exposure image of the point source. We can notice in Figure 3.2 (a) that instead of being a focused point of width approximately $\lambda / D$, the turbulence causes the long exposure image of the point source to be spread out and blurry spot. The width of the spot is around $0.7 \mu \mathrm{rad}$, which is the much larger than the diffraction limit case.

Short exposure images (of the order of few milliseconds) present different characteristics than the long exposure images. First, short exposure images have a broader extent than the diffraction limited image. Second, short exposure images are speckled in appearance [33]. They have a high spatial frequency component not present in long exposure images. We can observe this property in Figure 3.2 (b).

\subsection{Conclusion}

In this chapter, we saw how the fluctuations of the index of refraction affect wave propagation in the atmosphere. Two models for the spatial PSD of the index of refraction had been introduced: the Kolmogorov and the von Karman spectrum. Then, several turbulence profiles, characterizing the turbulence strength as a function of the propagation distance, had been presented. A layered model for the atmosphere was given as well as the formulas for computing the weights and altitudes of each layer. The method for propagation from one layer to the next was then explained. Finally, we illustrated 


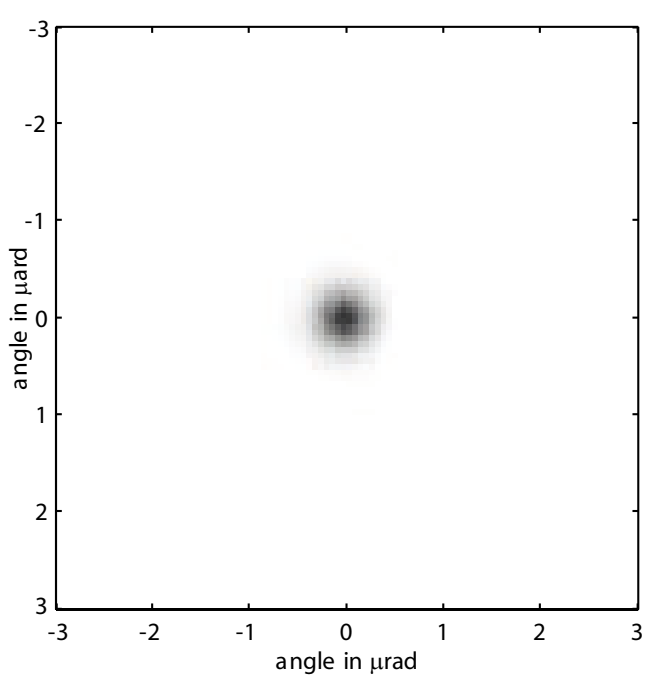

(a)

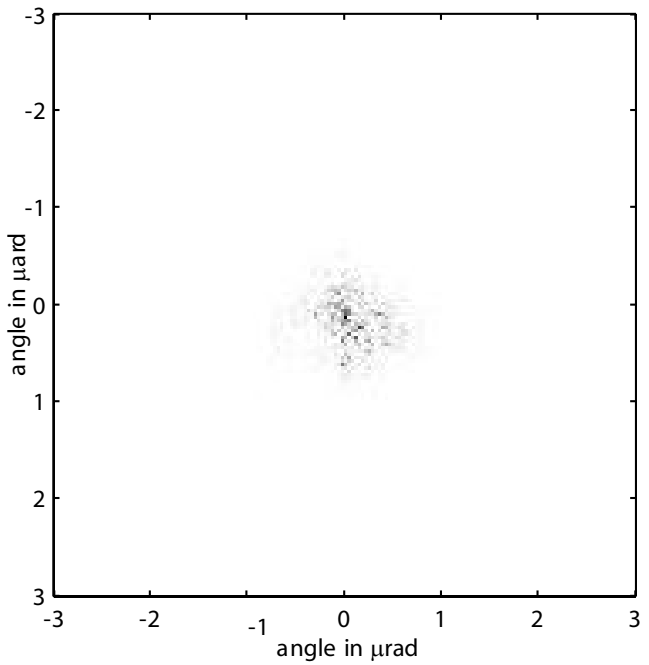

(b)

Figure 3.2: Image of a point source through turbulence. (a) Long exposure image. (b) Short exposure image. Negative image is displayed for clarity.

the effect of turbulence by observing the image of a point source through turbulence. 


\section{CHAPTER 4}

\section{Adaptive Optics}

\subsection{Introduction}

The goal of adaptive optics ( $\mathrm{AO})$ is to compensate turbulence-induced phase aberrations when propagating a light wave through the atmosphere. The idea consists of two steps: first, sense the phase aberrations, second, use this information to compensate for the phase deformation in real-time. In astronomical applications, an image is formed with the AO-corrected light waves. The image appears sharper thanks to adaptive optics. In communication or defense applications, laser beams are usually used. Adaptive optics allows to obtain more focused laser beams and therefore more power falling on the target. In this chapter, we will see the approach used in adaptive optics systems, as well as the actual components used in those systems. The performance and the limitations of adaptive optics will then be studied.

\subsection{Approach}

The principle of $\mathrm{AO}$ is the following: First, we get optical information from the incoming light. For example, we estimate the phase of the incoming field with a wavefront sensor. Second, we use an optical component, called deformable mirror (DM), to apply a correction to the beam. Sometimes, several DM's can be used [34, 35], but we 
only consider the case of a single DM. The DM is controlled in real-time by a computer processing the data from the wavefront sensor. To do so, closed-loop control is involved. The system adapts in real-time the DM to the optical distortion. The resulting light wave is then corrected. That is why the system is called adaptive optics.

\subsection{Adaptive optics components}

In this section, we present the structure of an adaptive optical imaging system. This kind of system is used in large ground-based telescopes. Figure 4.1 presents the organization of a typical adaptive optics system. In the next subsections, these components are described in detail.

\subsubsection{Wavefront sensor}

\section{Model}

The wavefront sensor (WFS) gives an estimate of the phase of the incoming optical field. This information is then used to control the wavefront compensation device, typically a deformable mirror (DM). Deformable mirrors will be studied in section 4.3.2. The difficulty of estimating the phase $\phi(\vec{x}, t)$ lies in the fact that it cannot be measured directly. Only the spatial gradient $\nabla \phi(\vec{x}, t)$ can be estimated. A phase reconstruction

algorithm is used to obtain an estimate of $\phi(\vec{x}, t)$, called $\hat{\phi}(\vec{x}, t)$. The phase reconstruction issue will be presented in section 4.3.3.

There are different types of wavefront sensors. The most commonly used is the Hartmann WFS. Figure 4.2 presents the optical configuration of the Hartmann wavefront sensor.

The Hartmann WFS works as follows. The incident light falls on the lenslet array 


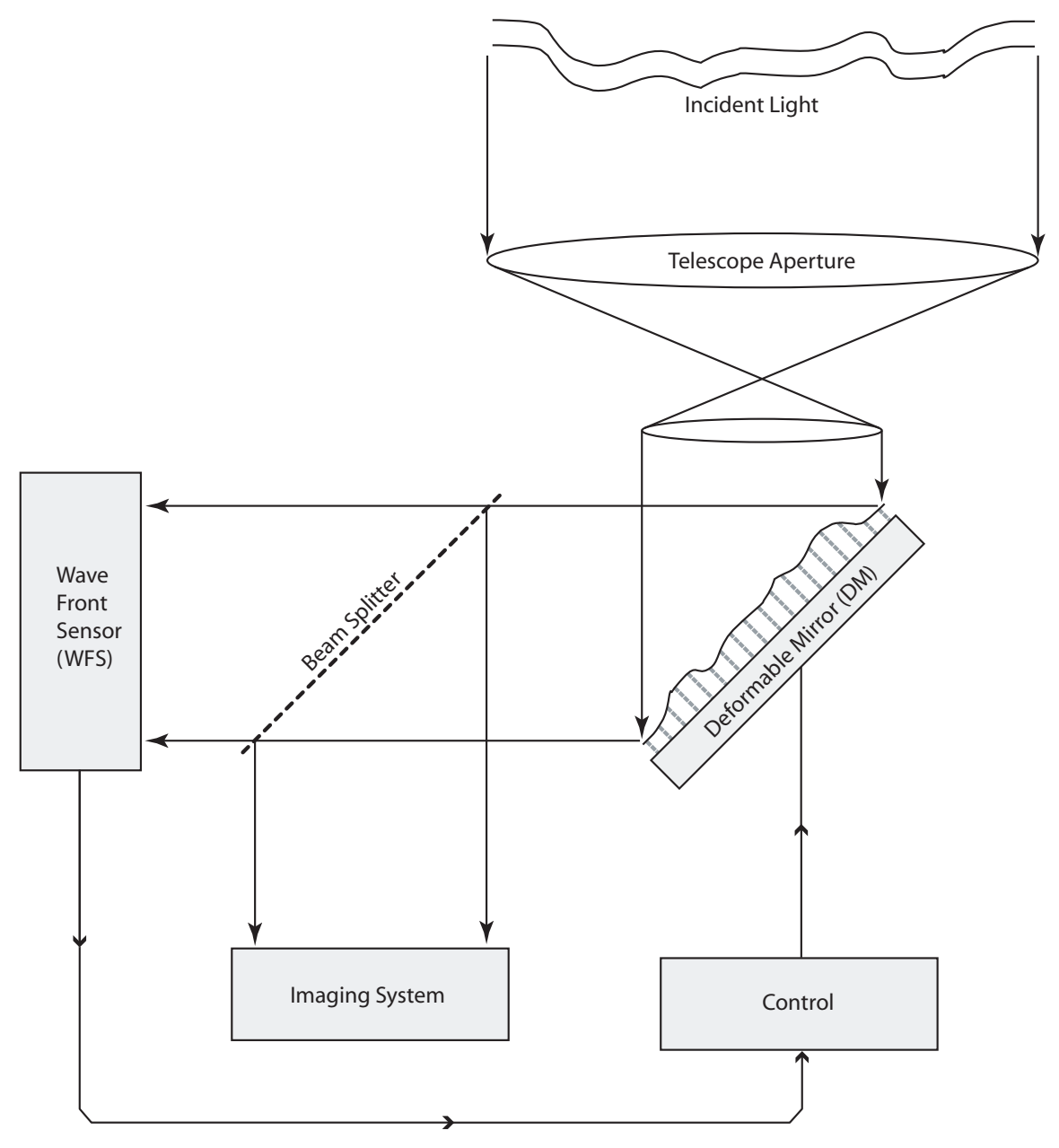

Figure 4.1: Organization of an adaptive optics system.

and each lenslet makes the light focus in the lenslet focal plane. An array of detectors is placed in the focal plane of the lenslet array to measure the position of the focusing spots. Each lenslet is also called subaperture. For each subaperture $i$, the wavefront slope $\vec{s}_{i}$ is proportional to the position of the spot $\vec{x}_{s p o t, i}$ in the focal plane. The relationship is given by

$$
\overrightarrow{s_{i}}=k \frac{\left|\vec{x}_{s p o t, i}\right|}{f},
$$

where $k$ is the wave number defined by $k=2 \pi / \lambda$, and $f$ the focal length of the lenslet. The location of the spot is estimated by calculating the centroid of the spot falling 

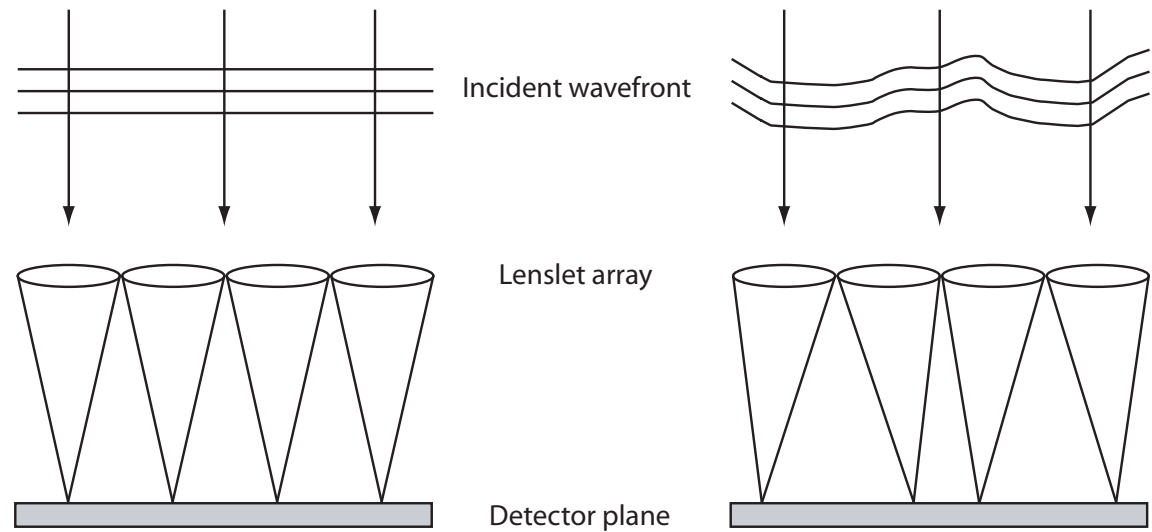

Lenslet array
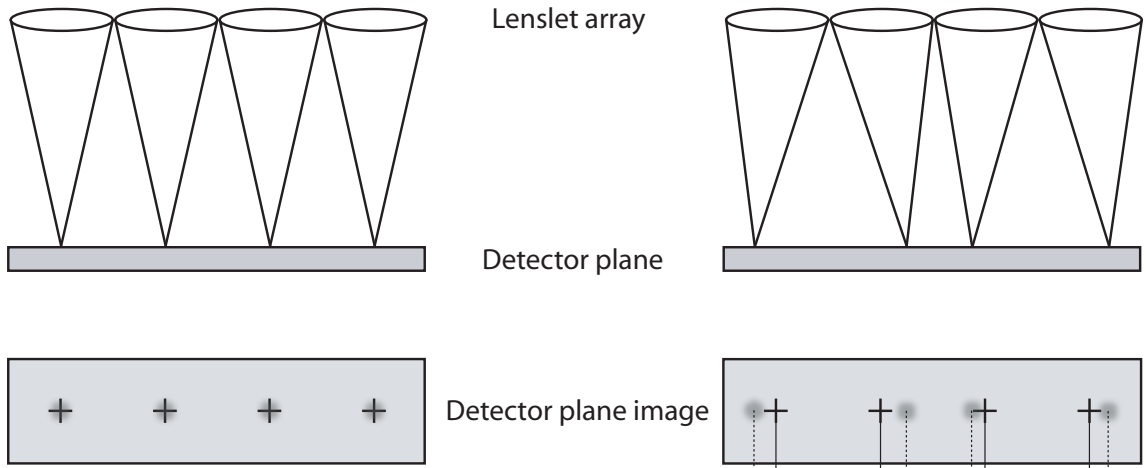

Detector plane image

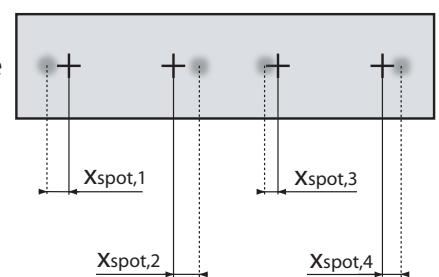

Figure 4.2: Hartmann wavefront sensor.

on the detector array. The relationship between the wavefront slope and the phase gradient over the $i^{\text {th }}$ subaperture is [15]

$$
\overrightarrow{s_{i}}=\frac{\int W_{\text {pupil }, i}(\vec{x}) \nabla \phi(\vec{x}, t) d \vec{x}}{\int W_{\text {pupil }, i}(\vec{x}) d \vec{x}}+\vec{s}_{n o i s e},
$$

where $W_{\text {pupil }, i}(\vec{x})$ is the weighting function of the $i^{t h}$ subaperture and $\nabla$ the notation for the gradient operator. The measurement noise is denoted $\vec{s}_{\text {noise }}$. Integrating by parts the numerator, we obtain

$$
\overrightarrow{s_{i}}=-\frac{\int \nabla W_{\text {pupil }, i}(\vec{x}) \phi(\vec{x}, t) d \vec{x}}{\int W_{\text {pupil }, i}(\vec{x}) d \vec{x}}+\vec{s}_{\text {noise }} .
$$

\section{Measurement errors}

We now focus our attention on quantifying the measurement errors when estimating the slope. The noise term $\vec{s}_{\text {noise }}$ in Eq. 4.2 has two components:

$$
\vec{s}_{\text {noise }}=\vec{s}_{n s}+\vec{s}_{n r},
$$


where $\vec{s}_{n s}$ is the shot noise, and $\vec{s}_{n r}$ is the read noise. The variances of $\vec{s}_{n s}$ and $\vec{s}_{n r}$ are designated $\sigma_{n s}^{2}$ and $\sigma_{n r}^{2}$. Thus, the variance of $\vec{s}_{n o i s e}$ is defined by:

$$
\sigma_{\text {noise }}^{2}=\sigma_{n s}^{2}+\sigma_{n r}^{2}
$$

The expressions of both the shot noise $[36,37]$ and the read noise can be found in the literature. In the case of the Hartmann WFS, the contribution to $\sigma_{\text {noise }}$ due to shot noise is written [15] (in radians $/ \mathrm{m}$ )

$$
\begin{aligned}
\sigma_{n s} & =k\left(\frac{\sigma_{\text {spot }}}{f}\right) \\
& =\frac{\sqrt{2} \pi}{d \sqrt{\bar{K}} \int_{-1}^{1} I_{b}\left(f_{x}, 0\right) H_{t r}\left(f_{x}, 0\right) d f_{x}},
\end{aligned}
$$

where $\sigma_{\text {spot }}$ is the standard deviation of the measurement of the spot location, $k$ is the wave number,

$f$ is the focal length of the lenslet, $d$ is the subaperture diameter,

$\bar{K}$ is the total average detected photon count per subaperture,

$I_{b}\left(f_{x}, f_{y}\right)$ is the Fourier transform of the light intensity distribution into the subaperture focal plane. $I_{b}\left(f_{x}, f_{y}\right)$ is normalized such that

$I_{b}(0,0)=1$.

$H_{t r}\left(f_{x}, f_{y}\right)$ is the tilt removed optical transfer function (OTF) of the subaperture,

finally $f_{x}$ and $f_{y}$ are the spatial frequency variables, which are normalized by the diffraction limit of the subaperture, $d / \lambda f$. This way, $f_{x} \in[-1 ; 1]$ and $f_{y} \in[-1 ; 1]$.

In Eq. 4.7, we can see that the variance $\sigma_{n s}$ is inversely proportional to the factor $\bar{K}$. This shows how the finite light level, represented by the variable $\bar{K}$, affects the accuracy of the slope measurements. Also, the influence of $\int_{-1}^{1} I_{b}\left(f_{x}, 0\right) H_{t r}\left(f_{x}, 0\right) d f_{x}$ on $\sigma_{n s}$ can 
be interpreted as follows: when the tilt-removed OTF if the subaperture, $H_{t r}\left(f_{x}, 0\right)$, gets closer to the diffraction limited OTF, the integral $\int_{-1}^{1} I_{b}\left(f_{x}, 0\right) H_{t r}\left(f_{x}, 0\right) d f_{x}$ tends to its maximum value of unity, and therefore, $\sigma_{n s}$ decreases. A lower bound for the measurement error due to shot or photon noise can then be defined (in radians $/ \mathrm{m}$ ):

$$
\sigma_{n s}=\frac{\sqrt{2} \pi}{d \sqrt{\bar{K}}}
$$

The second source of noise on the measurement of $\vec{s}$ is the light detector reading error. This additive noise is called the read noise. In the case of a quad cell, the contribution $\sigma_{n r}$ of the read noise to $\sigma_{n o i s e}$ can be expressed (in radians $/ \mathrm{m}$ ) by [9]

$$
\sigma_{n r}=\frac{\sqrt{2} \pi}{d \sqrt{\frac{\bar{K}^{2}}{4 \sigma_{e}^{2}}}}
$$

where $\sigma_{e}^{2}$ is the variance of the read noise for each pixel in the detector array.

\subsubsection{Wavefront compensation}

Wavefront aberrations $\phi(\vec{x}, t)$ can be represented as a linear combination of the influence functions of the DM:

$$
\phi(\vec{x}, t)=\sum_{k=1}^{N_{a c t}} a_{k}(t) r_{k}(\vec{x}),
$$

where $r_{k}(\vec{x})$ is the influence function of the $k^{t h}$ actuator, $t$ denotes the time, and $\vec{x}$ the position on the DM. The coefficient $a_{k}(t)$ is the weight of the $k^{t h}$ influence function in the decomposition of $\phi(\vec{x}, t)$. $N_{\text {act }}$ is the number of actuators of the DM. The weights $a_{k}(t)$ are given by

$$
a_{k}(t)=\int W_{\text {pupil }}(\vec{x}) \phi(\vec{x}) r_{k}(\vec{x}) d \vec{x},
$$

where $W_{\text {pupil }}(\vec{x})$ is a weight function corresponding to the pupil extent. $W_{\text {pupil }}(\vec{x})$ is defined by

$$
W_{\text {pupil }}(\vec{x})= \begin{cases}1 & \text { inside the pupil } \\ 0 & \text { outside the pupil. }\end{cases}
$$


A set of functions used to represent fixed wavefront aberrations is the Zernike polynomials. Using Zernike polynomials, the distorted phase, $\phi(\vec{x})$, can be expressed in polar coordinates as

$$
\phi(R \rho, \theta)=\sum_{n=1}^{N} a_{n} Z_{n}(\rho, \theta),
$$

where $R$ is the radius of the telescope aperture, $\rho$ and $\theta$ are the polar coordinates, $Z_{n}(\rho, \theta)$ is the $n$th Zernike polynomial, and $a_{n}$ 's are given by

$$
a_{n}=\int W_{\text {pupil }}(\rho) \phi(R \rho, \theta) Z_{n}(\rho, \theta) d \vec{\rho}
$$

Table 4.1 shows the first Zernike polynomials from $n=1$ to 10 . The first term, for $n=1$, is called piston, which is not been compensated in adaptive optics systems because it has not effect on the performances of imaging systems. The terms 2 and 3 are referred to as tilt. These are compensated by the use a tilt mirror, described in section 4.3.2.1. Higher order aberrations are compensated by the deformable mirror, which is discussed in section 4.3.2.2. DM's with localized actuators have localized influ-

\begin{tabular}{|r|l|}
\hline $\mathrm{n}$ & Zernike polynomial $Z_{n}(\rho, \theta)$ \\
\hline 1 & 1 \\
2 & $2 \rho \cos \theta$ \\
3 & $2 \rho \sin \theta$ \\
4 & $3.464 \rho^{2}-1.732$ \\
5 & $2.449 \rho^{2} \sin 2 \theta$ \\
6 & $2.449 \rho^{2} \cos 2 \theta$ \\
7 & $\left(8.485 \rho^{3}-5.657 \rho\right) \sin \theta$ \\
8 & $\left(8.485 \rho^{3}-5.657 \rho\right) \cos \theta$ \\
9 & $2.828 \rho^{3} \sin 3 \theta$ \\
10 & $2.828 \rho^{3} \cos 3 \theta$ \\
\hline
\end{tabular}

Table 4.1: Zernike polynomials. 
ence functions. Therefore, localized modes are an efficient representation, as opposed to Zernike polynomials, which are global. For that reason, despite its convenience to represent optical phase distortions, we prefer not to use Zernike polynomials in the rest of this thesis. Additionally, representing fine spatial features generally requires a huge number of Zernike polynomials.

\subsubsection{Tilt mirror}

Most of the power of the wavefront phase distortion is in the lower dynamic range $(n$ $=2$ and $n=3$ ) [15]. The tilt aberration does not cause an image defect. Rather, it causes the image location to shift. If multiple realizations of tilt are integrated in an image exposure time, a blur results. For this reason, we use a tilt mirror (also called tip-tilt mirror) to compensate for the tilt aberrations of the wavefront $[38,39]$. The incoming light bounces first on the tilt mirror, and then on the deformable mirror. A tilt mirror is simply a flat mirror with two degrees of freedom on axis $x$ and $y$. Figure 4.3 presents the aspect of a tip-tilt mirror. The effect of a wavefront tilt is to

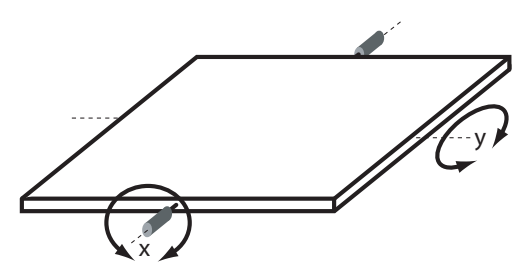

Figure 4.3: Tip-tilt mirror.

keep the image centered in the focal plane of the imaging system. The tilt is estimated by computing the centroid of the image. The centroid of the intensity distribution $I(x, y)$ is calculated by:

$$
\begin{aligned}
\bar{x} & =\iint I(x, y) x d x d y \\
\bar{y} & =\iint I(x, y) y d x d y .
\end{aligned}
$$


The tilt compensation is controlled by a closed-loop system. The command signals sent to the two orthogonal axis $x$ and $y$ of the tip-tilt mirror are computed from the centroid position and updated in real-time. Tyler [40] defined the minimum temporal frequency $f_{T}$ required for tilt compensation in the case of a circular aperture. $f_{T}$ is also called the Greenwood frequency and is defined by

$$
f_{T}=0.368 D^{-1 / 6} \lambda^{-1}\left[\int C_{n}^{2}(z) v^{2}(z) d z\right]^{2}
$$

where $D$ is the diameter of the pupil, $\lambda$ the wavelength, $C_{n}^{2}(z)$ the turbulence profile, and $v(z)$ the turbulence velocity profile. Tyler also found the expression of the variance of the residual angular tilt after compensation. This variance is called $\sigma_{\theta}^{2}$ and is written

$$
\sigma_{\theta}^{2}=\left(\frac{f_{T}}{f_{3 d B}}\right)^{2}\left(\frac{\lambda}{D}\right)^{2}
$$

where $f_{3 d B}$ is the temporal frequency such that the system response is $3 \mathrm{~dB}$ down from its maximum value.

\subsubsection{Deformable mirror}

The deformable mirror (DM) corrects for the higher spatial orders of the wavefront aberrations. The aberrated incident wavefront falls on the mirror and is reflected such that the wavefront phase is, at least partially, cancelled. There are two types of deformable mirror: segmented and continuous. More details about both types of DM can be found in $[15,41,42,43,44]$. A continuous DM has a single, thin, continuous mirror surface that can be shaped to the desired pattern. The shape of the mirror is adjusted by a array of discrete electronically controled actuators that push and pull the face sheet (See Figure 4.4). In Eq. 4.10 we modeled the surface of the DM. The influence functions $r_{k}(\vec{x})$ had been modeled using different functions: polynomials, trigonometric and Gaussian functions [45, 46, 47, 48, 49]. In the following section, we explain how the control signal $c_{k}(t)$ is calculated. It should be noted that due to the 
nature of the surface, the actuators are mechanically coupled by the face sheet, and the influence functions associated with different actuators are usually not independent.

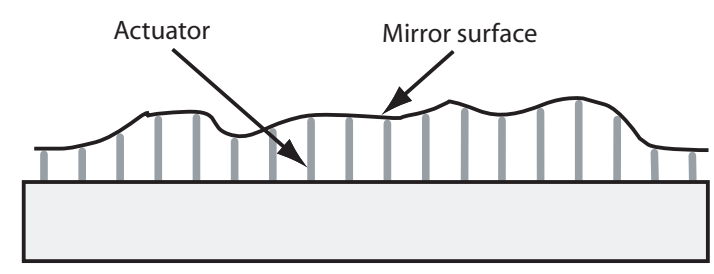

Figure 4.4: Cross-section of a deformable mirror.

\subsubsection{Control}

We already established the relationship between the actuator commands and the DM surface in Eq. 4.10. We now want to compute the DM command $a_{k}(t)$ for each actuator indexed by $k$. The WFS slope measurements $\vec{s}_{\text {meas }, i}(t)$ for each subaperture $i$ are used to compute the DM commands. This is called mapping the slope measurements to the DM commands. The goal is to build a mapping that minimizes the squared residual phase averaged over the aperture, $\epsilon_{\text {res }}$, defined by

$$
\epsilon_{\text {res }}^{2}=\int W_{\text {pupil }}(\vec{x})(\hat{\phi}(\vec{x}, t)-\phi(\vec{x}, t))^{2} d \vec{x}
$$

Let's call $\mathbf{c}$ and $\mathbf{s}$ the vector columns built respectively from the $c_{k}(t)$ 's and the $\vec{s}_{\text {meas }, i}(t)$ 's. For a linear mapping between the WFS measurements and the DM commands, Roggemann and Welsh [15] gave the following expression:

$$
\mathbf{c}=\mathbf{M s}
$$

where $\mathbf{M}$ is a $N_{\text {act }} \times 2 N_{\text {subap }}$ matrix called the control matrix, where $N_{\text {act }}$ and $N_{\text {subap }}$ are respectively the number of actuators and subapertures. The matrix $\mathbf{M}$ is derived by minimizing the slope measurement error. Assuming that the measurement error follows 
a Gaussian distribution, the matrix $\mathbf{M}$ can be calculated using different estimators. The most commonly used estimators are: the maximum a posteriori (MAP) estimator, the minimum variance estimator, the maximum likelihood estimator, and the least squares (LS) estimator. In this section, we give details about the MAP estimator as well as the LS estimator, which is the type of estimator we use later on.

\section{Maximum a posteriori (MAP) estimator}

The following model is used for the slope measurements:

$$
\mathbf{s}_{\text {meas }}=\mathbf{s}_{d m}+\mathbf{s}_{\text {noise }},
$$

where $\mathbf{s}_{d m}$ is the column vector containing the slopes of the DM surface, and $\mathbf{s}_{\text {noise }}$ the column vector containing the noise measurements (due to both shot noise and read noise). From Eq. 4.3, we can write:

$$
s_{d m, j}=-\frac{\int\left(\nabla W_{\text {pupil }, j}(\vec{x}) \cdot \overrightarrow{u_{j}}\right) \hat{\phi}(\vec{x}, t) d \vec{x}}{\int W_{\text {pupil }, j}(\vec{x}) d \vec{x}},
$$

where $s_{d m, j}$ is the $j$ th element of the vector $\mathbf{s}_{d m}$ and $\overrightarrow{u_{j}}$ is a unit direction vector. We now look only at an instant of time, so we dismiss the time variable $t$. Substituting Eq. 4.13 in Eq. 4.22 we have:

$$
s_{d m, j}=-\sum_{k}^{N_{\text {act }}} c_{k} \frac{\int\left(\nabla W_{\text {pupil }, j}(\vec{x}) \cdot \overrightarrow{u_{j}}\right) r_{k}(\vec{x}) d \vec{x}}{\int W_{\text {pupil }, j}(\vec{x}) d \vec{x}} .
$$

Let now define the element $(m, n)$ of the Jacobian matrix $H$ as

$$
H_{i j}=-\frac{\int\left(\nabla W_{\text {pupil }, j}(\vec{x}) \cdot \overrightarrow{u_{j}}\right) r_{k}(\vec{x}) d \vec{x}}{\int W_{\text {pupil }, j}(\vec{x}) d \vec{x}} .
$$

This allows us to write

$$
\mathbf{s}_{d m}=H \mathbf{c},
$$

where $\mathbf{c}$ is the DM commands column vector. Substituting Eq. 4.21 in Eq. 4.25 gives:

$$
\mathbf{s}_{\text {meas }}=H \mathbf{c}+\mathbf{s}_{\text {noise }} .
$$


Assuming that the noise $\mathbf{s}_{\text {noise }}$ has a Gaussian distribution and a zero-mean, we can establish (see Eq. 4.20) that $\mathbf{c}$ also has a zero-mean Gaussian distribution [26, 50]. Let define the covariance matrices of $\mathbf{c}$ and $\mathbf{s}_{\text {noise }}$

$$
\begin{aligned}
C_{c} & =E\left\{\mathbf{c c}^{T}\right\}, \\
C_{\text {noise }} & =E\left\{\mathbf{s}_{\text {noise }} \mathbf{s}_{\text {noise }}^{T}\right\} .
\end{aligned}
$$

If the influence functions $r_{i}(\vec{x})$ are orthonormal, and if the input phase $\phi(\vec{x})$ is zeromean, the coefficients of the control signal covariance matrix $C_{c}$ are given by

$$
\begin{aligned}
\bar{c}_{i} & =E\left\{\int W(\vec{x}) \phi(\vec{x}) r_{i}(\vec{x}) d \vec{x}\right\}, \\
& =\int W(\vec{x}) E\{\phi(\vec{x})\} r_{i}(\vec{x}) d \vec{x}, \\
& =0
\end{aligned}
$$

and

$$
\begin{aligned}
c_{i} \bar{c}_{j} & =E\left\{\int W(\vec{x}) \phi(\vec{x}) r_{i}(\vec{x}) d \vec{x} \int W\left(\overrightarrow{x^{\prime}}\right) \phi\left(\overrightarrow{x^{\prime}}\right) r_{j}\left(\overrightarrow{x^{\prime}}\right) d \overrightarrow{x^{\prime}}\right\} \\
& =\iint W(\vec{x}) W\left(\overrightarrow{x^{\prime}}\right) r_{i}(\vec{x}) r_{j}\left(\overrightarrow{x^{\prime}}\right) E\left\{\phi(\vec{x}) \phi\left(\overrightarrow{x^{\prime}}\right)\right\} d \vec{x} d \overrightarrow{x^{\prime}}
\end{aligned}
$$

The atmosphere statistics enter in Eq. 4.30 by means of the correlation function $\Gamma_{\phi}\left(\vec{x}, \overrightarrow{x^{\prime}}\right)=E\left\{\phi(\vec{x}) \phi\left(\overrightarrow{x^{\prime}}\right)\right\}$. The MAP estimate minimizes the term $\left\|\mathbf{s}_{d m}-\mathbf{s}_{\text {meas }}\right\|^{2}$. Reference [51] gives the result of this minimization:

$$
\mathbf{c}_{M A P}=\left(\mathbf{H}^{T} C_{\text {noise }}^{-1} \mathbf{H}+C_{c}^{-1}\right)^{-1} \mathbf{H}^{T} C_{\text {noise }}^{-1} \mathbf{s}_{\text {meas }} .
$$

Comparing Eq. 4.20 and Eq. 4.31, we recognize the expression of the control matrix $\mathbf{M}$ in the case of the MAP estimation:

$$
\mathbf{M}_{M A P}=\left(\mathbf{H}^{T} C_{\text {noise }}^{-1} \mathbf{H}+C_{c}^{-1}\right)^{-1} \mathbf{H}^{T} C_{\text {noise }}^{-1}
$$




\section{Least squares (LS) estimator}

As the measurement noise variance decreases to zero, Eq. 4.32 reduces to the LS control matrix. In the LS estimate, the quantity $\left\|\mathbf{s}_{\text {meas }}-\mathbf{H c}\right\|^{2}$ is being minimized. The solution to this is given by [52]:

$$
\mathbf{c}_{L S}=\left(\mathbf{H}^{T} \mathbf{H}\right)^{-1} \mathbf{H}^{T} \mathbf{s}_{\text {meas }} .
$$

In which we recognize the expression of the control matrix $\mathbf{M}$ in the case of the LS estimate:

$$
\mathbf{M}_{L S}=\left(\mathbf{H}^{T} \mathbf{H}\right)^{-1} \mathbf{H}^{T}
$$

The error between the DM phase and the desired phase is never zero. This error depends on several factors: the noise in the WFS measurements (see Eq. 4.7 and Eq. 4.9), the finite number of DM actuators and the spacing between them, the size and the sampling of the WFS subapertures and the anisoplanatism effect. We can notice that the LS estimator technique does not need any atmosphere statistics, which are required by the MAP estimator. Thus, for this reason, as well as for its simplicity, the LS estimator is the most widely used in practice at this time.

\subsection{Performance measures}

The degree of success of AO systems in compensating the wave front phase aberration depends on:

1. The components of the system (See section 4.3),

2. The atmospheric conditions,

3. The light levels (See section 4.5). 
There are many ways to measure AO systems performances [5, 47, 48, 53, 54]. The Strehl ratio (SR) is simple and commonly used metric. It is defined by

$$
S R=\frac{h(\overrightarrow{0})}{h_{d l}(\overrightarrow{0})},
$$

where $h(\vec{x})$ is the point spread function (PSF) and $h_{d l}(\vec{x})$ the diffraction limited PSF. It also can be expressed as the ratio of the integrals of the optical function transfer (OTF), $H(\vec{f})$, and $H_{d l}(\vec{f})$ :

$$
S R=\frac{\int H(\vec{f}) d \vec{f}}{\int H_{d l}(\vec{f}) d \vec{f}}
$$

If the aperture averaged residual wavefront phase $\epsilon_{\text {res }}^{2}$, as defined in Eq. 4.19, is less than $(2 \pi / 10)^{2} \operatorname{rad}^{2}$, an approximation of the Strehl ratio, known as the Marechal approximation [55], is given by

$$
S R \approx \exp \left\{-\epsilon_{\text {res }}^{2}\right\} .
$$

\subsection{Factors limiting performance}

\subsubsection{WFS light level}

The finite amount of light falling onto the WFS is a limiting factor for AO systems. The influence of this parameter on the WFS measurements accuracy had been quantified in 4.3.1. In astronomy, most of the time the science object is not bright enough to be used a reference beacon for the $\mathrm{AO}$ system. To remedy this problem, a bright star should be found nearby the object. However, the bright star should not have a large angular separation with the object, which leads me to the next section on anisoplanatism. 


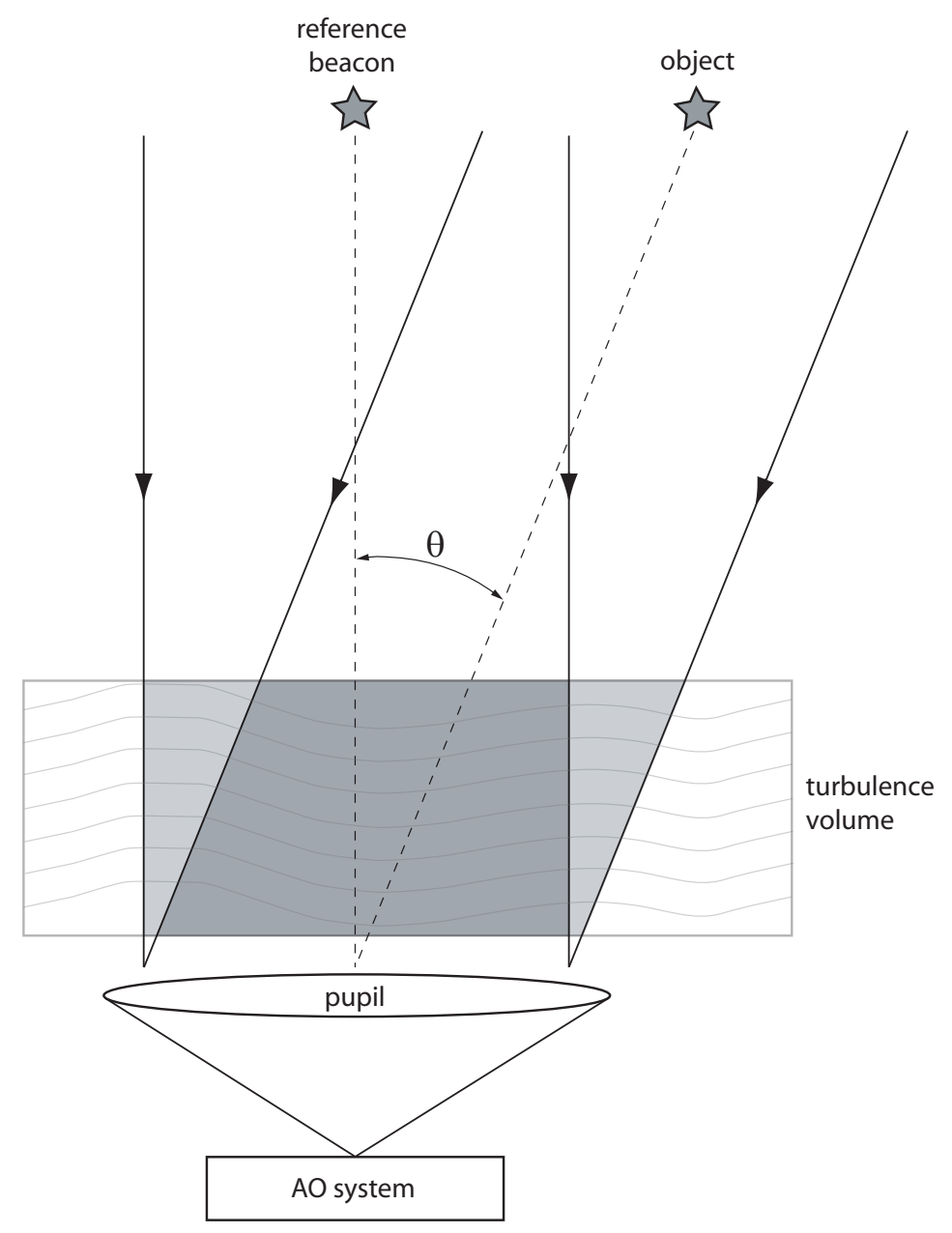

Figure 4.5: Anisoplanatism.

\subsubsection{Anisoplanatism}

Anisoplanatism arises from the fact that the light coming from different directions does not go through the same volume of turbulence, and thus does not experience the same phase aberration. Figure 4.5 illustrates this idea. To quantify this phenomenon, the isoplanatic angle had been defined. The isoplanatic angle is the maximum angular separation between the object we look at and the reference beacon for which the phase aberration remains approximately constant. Fried [10] defined the isoplanatic angle as the angle between the beacon and object paths for which the average far field transmit 
power will be reduced from its maximum value by a factor of $1 / e$. It is written by

$$
\theta_{0}=58.1 \times 10^{-3} \lambda^{6 / 5}\left[\int_{0}^{L} C_{n}^{2}(z) z^{5 / 3} d z\right]^{-3 / 5}
$$

where $C_{n}^{2}(z)$ is the turbulence profile, $\lambda$ the wavelength, and $L$ the path length through turbulence. We can notice that $\theta_{0}$ depends only on $C_{n}^{2}(z)$ and $\lambda$, and not at all on the AO system itself. The isoplanatic angle $\theta_{0}$ can also be defined as a function of the Fried parameter $r_{0}$ :

$$
\theta_{0}=0.314 \frac{r_{0}}{\bar{h}}
$$

where $r_{0}$ was defined in Eq. 3.12 and $\bar{h}$ is defined by

$$
\bar{h}=\left[\frac{\int_{0}^{L} C_{n}^{2}(z) z^{5 / 3} d z}{\int_{0}^{L} C_{n}^{2}(z) d z}\right]^{3 / 5} .
$$

The parameter $k$ is the wave number defined by $k=2 \pi / \lambda$. In Eq. 4.38, we can see the dependency on the wavelength $\lambda$. In the visible band, a typical value for $\theta_{0}$ would be 5-10 $\mu$ radians, but it would be much larger in the infrared (IR) wavelengths. In Eq. 4.39, we note that $\theta_{0}$ is proportional to $r_{0}$. The parameter $r_{0}$ is measurement of the strength of the turbulence. The stronger the turbulence is, the smaller $r_{0}$ becomes, and so $\theta_{0}$.

Thus, the performance of $\mathrm{AO}$ systems depends on the direction of the object of interest. When the angular separation between the object and the reference beacon increases, the performance of the AO system degrades [12]. In the 90's, the problem of finding a bright enough natural star to use as a reference beacon was solved by the creation of an artificial guide star $[9,11]$. By forming a laser beam in the upper atmosphere, a spot is created and can be used as reference beacon for the AO system. This way, the problem of the low light level in the WFS is eliminated. However, artificial beacons do not provide any tilt information. By pointing the laser beam within the isoplanatic angle of the object to observe, we avoid the disadvantage due to anisoplanatism. However, 
the problem remains when considering wide field of view images. This point will be treated in the next chapter.

\subsubsection{Technical limitations}

The other limitations of the AO systems are due to technical constrains:

- First, there can be only a finite number of actuators deforming the DM. This results in a finite degrees-of-freedom of the DM, which limits the range of spatial frequencies that the mirror can compensate.

- Second, the finite sample spacing in the WFS limits the ability to sense high spatial frequencies in the wavefront phase. The finite number of DM actuators and the finite sample spacing in the WFS both act as a spatial filter on the phase reconstructed by the DM surface.

- Finally, AO system performance is limited from the fact that wavefront aberration evolves in time. The sensing of the wavefront aberration, as well as the DM deformation takes a time delay. This time delay limits the temporal response of the system, as well as its performances.

\subsection{Conclusion}

In this chapter, we introduced the approach used in AO systems to compensate turbulenceinduced phase aberration. We described each component of the system, and gave its model. Tools used to measure performance of AO systems were presented. Finally, we studied how anisoplanatism, as well as the light level in the WFS, limit the performance of AO systems. 


\section{CHAPTER 5}

\section{Simulations}

\subsection{Introduction}

In this chapter, we explain why wave optics computer simulations are used and we give the details of the simulation used here.

Stochastic processes lead the atmospheric turbulence phenomenon. Therefore, when considering the problem of propagating light through the atmosphere, analytic solutions are either impossible, or very hard to calculate. For this reason, we use Monte Carlo simulations [56]. Numerical simulations are used to first propagate the optical field through the atmosphere, and then to form the image, accounting for the action of the adaptive optical system [54].

The Fourier optics concepts presented in chapter 2 as well as the models of the components of the $\mathrm{AO}$ imaging system introduced in chapter 4 are implemented in the simulation.

This chapter is organized as follows. First, we present Monte Carlo simulations in general. Second, we describe the sampling requirements needed when implementing wave propagation simulations. Third, the parameters of the wave optics simulation are listed. The last section of this chapter is a conclusion. 


\subsection{Simulations}

\subsubsection{Monte Carlo simulations}

Since atmospheric turbulence is a stochastic process, we use Monte Carlo simulations [56]. The wave propagation simulation is run several times, each time with a different random realization of the phase screens. This way, we generate random realizations of the wave front phase falling onto the pupil of the imaging system. The image is then formed with the AO imaging system. The resulting images are averaged over all the iterations. Typically, the simulation is run a few hundred times in order to obtain long exposure images.

Different software packages offer toolboxes for optics wave propagation simulations [57]. I decided to use the computing software Matlab [58] and the toolbox AOTools [59] to perform the simulations. Matlab offers a very flexible environment and the simulation program can easily be modified. The AOTools toolbox provides very convenient tools to:

- Model adaptive optics systems,

- Propagate the optical wave through random media,

- Simulate imaging systems,

- Analyze the propagated optical field.

\subsubsection{Array size}

The discretized values of the optical field are stored in two-dimensional complex matrices. Each matrix corresponds to the field in a plane denoted $\left.(x, y)\right|_{z=Z}$, where $Z$ is 
the propagation distance. The plane $\left.(x, y)\right|_{z=Z}$ is normal to the optical axis of the system. The size for the matrices depends on the sampling, which is driven by either the propagator, or the WFS sampling. A commonly used size for the matrices is $512 \times 512$, which is the size I used. For sizes larger than $1024 \times 1024$, simulations become slow due to the limited computational power of computers. The size of matrices is usually taken as a power of two $(128,256,512$, etc) since algorithms like the fast Fourier transform (FFT) or the Inverse Fast Fourier transform (IFFT) run much faster with powers of two.

\subsubsection{Phase screen generation}

\section{Approach}

In this section, we describe the method used to generate random realizations of the wavefront phase [15]. A realization of the wavefront phase at time $t$ can be writen as

$$
\tilde{\varphi}(\vec{x}, t)=\sum_{i=1}^{N} a_{i}(t) f_{i}(\vec{x}),
$$

where $\tilde{\varphi}(\vec{x}, t)$ is a single realization of the random phase screen, $\vec{x}$ is the vector position in the pupil plane, $a_{i}(t)$ is the $i^{t h}$ weight at time instant $t$, and $f_{i}(\vec{x})$ is the $i^{t h}$ element of a set of orthonormal basis functions. A widely used set of orthonormal basis functions is the Zernike polynomials set. Another one is $f_{i}(\vec{x})=\delta\left(\vec{x}-\overrightarrow{x_{i}}\right)$, which is the one used in the AOTools. In the latter case, the basis function corresponds to the value of the random phase at position $\overrightarrow{x_{i}}$. The generation of random phase screens consists in finding random set of weights $a_{i}(t)$, for $i=1, \ldots, P$, respecting approximately the desired statistical properties of the phase [15]. The spatial and temporal correlation property should be respected:

$$
\Gamma_{\tilde{\varphi}}\left(\vec{x}, t ; \vec{x}^{\prime}, t^{\prime}\right) \approx \Gamma_{\varphi}\left(\vec{x}, t ; \vec{x}^{\prime}, t^{\prime}\right)
$$


where

$$
\begin{aligned}
\Gamma_{\tilde{\varphi}}\left(\vec{x}, t ; \vec{x}^{\prime}, t^{\prime}\right) & =E\left\{\tilde{\varphi}(\vec{x}, t) \tilde{\varphi}\left(\vec{x}^{\prime}, t^{\prime}\right)\right\} \\
\Gamma_{\varphi}\left(\vec{x}, t ; \vec{x}^{\prime}, t^{\prime}\right) & =E\left\{\varphi(\vec{x}, t) \varphi\left(\vec{x}^{\prime}, t^{\prime}\right)\right\}
\end{aligned}
$$

and $\varphi(\vec{x}, t)$ denotes the random phase we are simulating. First, let us consider the covariance matrix for the weights $a_{i}(t)$. We denote this matrix $\Gamma_{a}$. If we consider $M$ different instants of time $t_{k}$ and $N$ vectors position $\vec{x}_{i}, \Gamma_{a}$ has $(N \times M)^{2}$ elements. Each element is given by

$$
E\left\{a_{i}\left(t_{k}\right) a_{i^{\prime}}\left(t_{k^{\prime}}\right)\right\} \text { for }\left\{i, i^{\prime}\right\} \in[1, \ldots, N]^{2} \text {, and }\left\{k, k^{\prime}\right\} \in[1, \ldots, M]^{2}
$$

Since the matrix $\Gamma_{a}$ is real, symmetric and positive definite [60], the Choleski factorization allows us to write $\Gamma_{a}$ as the product of two square matrices [61]:

$$
\Gamma_{a}=R R^{T}
$$

where $T$ denotes the transpose operator. Numerical methods [61, 62] exist to compute this factorization.

We now consider a vector uncorrelated, zero mean, unit variance, and Gaussian random variables. This column vector is denoted $\vec{b}$ and had $M \times N$ elements. Random number generators [58] provide such random variable realizations. The covariance of the vector $\vec{b}$ is given by

$$
E\left\{\vec{b} b^{T}\right\}=I_{M}
$$

where $I_{M}$ is the identity matrix of size $M \times M$. Let us now form the vector $\vec{a}$ from the matrix $R$ and the vector $\vec{b}$ this way:

$$
\vec{a}=R \vec{b}
$$

where the elements of the vector $\vec{a}$ correspond to the weights $a_{i}\left(t_{k}\right)$. Then, we compute the statistics of the vector $\vec{a}[15]$ :

$$
E\left\{\vec{a} a^{T}\right\}=E\left\{R \vec{b}(R \vec{b})^{T}\right\}
$$




$$
=E\left\{R \vec{b} \vec{b}^{T} R^{T}\right\}
$$

Since $R$ is a constant matrix, we can write

$$
E\left\{\vec{a} \vec{a}^{T}\right\}=R E\left\{\vec{b}^{T}\right\} R^{T} .
$$

By substituting Eq. 5.7 into Eq. 5.11, we obtain

$$
\begin{aligned}
E\left\{\vec{a} \vec{a}^{T}\right\} & =R I_{M} R^{T} \\
& =R R^{T} \\
& =\Gamma_{a}
\end{aligned}
$$

Through these steps, we proved that the random vector $\vec{a}$ has the desired covariance matrix $\Gamma_{a}$.

\section{Covariance matrix $\Gamma_{a}$}

Now that we showed that we can obtain draws of the random vector $\vec{a}$ having the desired covariance $\Gamma_{a}$, we explain in this section how to compute the elements of the matrix $\Gamma_{a}$. Assuming that we take as a basis function set, $f_{i}(\vec{x})=\delta\left(\vec{x}-\overrightarrow{x_{i}}\right)$, Eq. 5.5 gives

$$
\begin{aligned}
E\left\{a_{i}\left(t_{k}\right) a_{i^{\prime}}\left(t_{k^{\prime}}\right)\right\} & =E\left\{\varphi\left(\overrightarrow{x_{i}}, t_{k}\right) \varphi\left(\overrightarrow{x_{i^{\prime}}}, t_{k^{\prime}}\right)\right\} \\
& =\Gamma_{\varphi}\left(\overrightarrow{x_{i}}, t_{k} ; \overrightarrow{i_{i^{\prime}}}, t_{k^{\prime}}\right)
\end{aligned}
$$

We can notice that the covariance matrix $\Gamma_{a}$ corresponds to the covariance expression for the phase, $\Gamma_{\varphi}$. The covariance matrix $\Gamma_{\varphi}$ depends on the turbulence model chosen. Ref. [15] gives the expression of $\Gamma_{\varphi}$ for different turbulence models. Let us first define the piston-removed phase $\phi(\vec{x}, t)$. Its expression is given by

$$
\phi(\vec{x}, t)=\varphi(\vec{x}, t)-\int W\left(\overrightarrow{x^{\prime}}\right) \varphi\left(\overrightarrow{x^{\prime}}, t\right) d \overrightarrow{x^{\prime}}
$$


where $W\left(\overrightarrow{x^{\prime}}\right)$ is the weighting function defining the physical extent of the phase screen.

In the case of the von Karman turbulence model, Ref. [15] gives the following expression for the covariance of the piston-removed phase $\phi(\vec{x}, t)$ :

$$
\Gamma_{\phi}\left(\overrightarrow{x_{i}}, t_{k} ; \overrightarrow{x_{i^{\prime}}}, t_{k^{\prime}}\right)=3.089\left(\sum_{n=1}^{N} r_{0_{n}}^{-5 / 3} \int_{0}^{\infty} \kappa J_{0}\left(\kappa\left|\overrightarrow{x_{i}}-\overrightarrow{x_{i^{\prime}}}-\vec{v}\left(z_{n}\right)\left(t_{k}-t_{k^{\prime}}\right)\right|\right) \Phi_{0}^{V}(\kappa) d \kappa\right),
$$

where $\kappa$ denotes the scalar wavenumber, $J_{0}($.$) denotes the zeroth order Bessel function$ of the first kind, $\vec{v}(z)$ is the velocity profile, $\Phi_{0}^{V}(\kappa)$ is the von Karman spectrum, $r_{0_{n}}$ is the Fried parameter for the $n$th layer and is defined by

$$
r_{0_{n}}=0.185\left[\frac{4 \pi^{2}}{k^{2} C_{n_{n}}^{2} \Delta z_{n}}\right]^{3 / 5},
$$

where $k$ is the optical wavenumber, $\Delta z_{n}$ the thickness of the $n$th layer, and $C_{n_{n}}^{2}$ is the structure constant for this layer. We can note that the Bessel function, $J_{0}($.$) , can be$ evaluated using already existing software toolboxes.

In the case of the piston-removed von Kolmogorov turbulence model, the covariance of the piston-removed phase $\phi(\vec{x}, t)$ is [15]

$$
\begin{aligned}
& \Gamma_{\phi}\left(\overrightarrow{x_{i}}, t_{k} ; \overrightarrow{x_{i^{\prime}}}, t_{k^{\prime}}\right)=6.88 \sum_{n=1}^{N} r_{0_{n}}^{-5 / 3}\left(-\frac{1}{2}\left|\overrightarrow{x_{i}}-\overrightarrow{x_{i^{\prime}}}-\vec{v}\left(z_{n}\right)\left(t_{k}-t_{k^{\prime}}\right)\right|^{5 / 3}\right. \\
& +\frac{1}{2} \int W\left(\overrightarrow{x^{\prime}}\right)\left|\overrightarrow{x^{\prime}}-\overrightarrow{x_{i^{\prime}}}-\vec{v}\left(z_{n}\right)\left(t_{k}-t_{k^{\prime}}\right)\right|^{5 / 3} d \overrightarrow{x^{\prime}} \\
& +\frac{1}{2} \int W\left(\overrightarrow{x^{\prime \prime}}\right)\left|\overrightarrow{x_{i}}-\overrightarrow{x^{\prime \prime}}-\vec{v}\left(z_{n}\right)\left(t_{k}-t_{k^{\prime}}\right)\right|^{5 / 3} d \overrightarrow{x^{\prime \prime}} \\
& \left.+\frac{1}{2} \iint W\left(\overrightarrow{x^{\prime}}\right) W\left(\overrightarrow{x^{\prime \prime}}\right)\left|\overrightarrow{x^{\prime}}-\overrightarrow{x^{\prime \prime}}-\vec{v}\left(z_{n}\right)\left(t_{k}-t_{k^{\prime}}\right)\right|^{5 / 3} d \overrightarrow{x^{\prime}} d \overrightarrow{x^{\prime \prime}}\right) .
\end{aligned}
$$

In this section, we showed how to generate random phase screens. Figure 5.1 shows a realization of a $512 \times 512$ pixels phase screen computed with the Kolmogorov spatial power spectrum, for $D / r_{0}=25$. Eight turbulence layers are used as well as a zero velocity profile. 


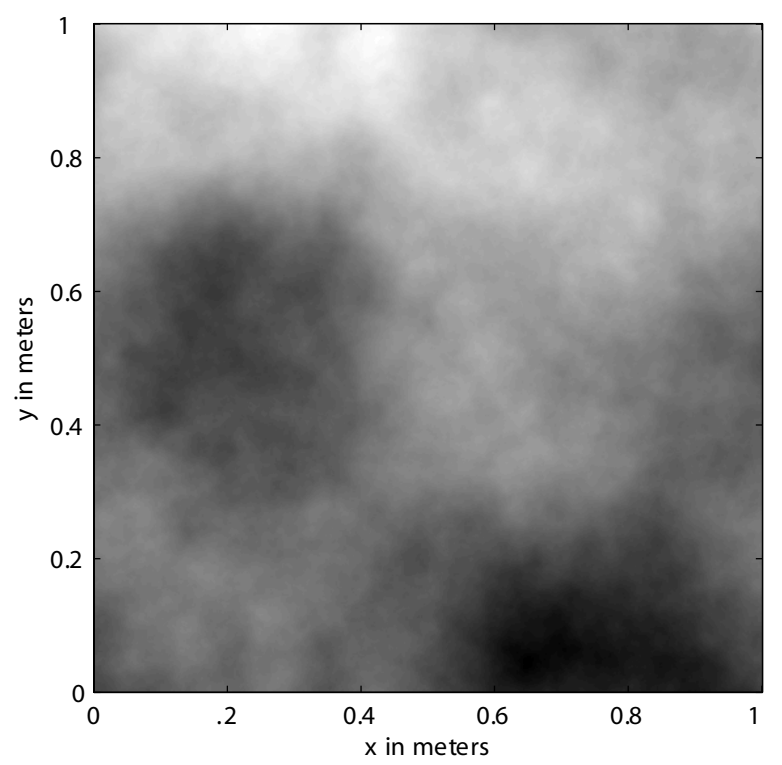

Figure 5.1: Example of a phase screen computed with the Kolmogorov spatial power spectrum. Black pixels correspond to $-\pi$ and white to $\pi$ radians.

\subsection{Simulation parameters}

\subsubsection{Propagation parameters}

The parameters of the simulation concerning the propagation through the atmosphere are presented in Table 5.1.

\subsubsection{Telescope}

The parameters of the simulation concerning the telescope are presented in Table 5.2.

Figure 5.2 shows an example of simulated intensity image in the pupil plane of the telescope. The primary and the secondary apertures can be seen, as well as the "spider" of the telescope. 


\begin{tabular}{|l|l|}
\hline Type of simulation & Vertical imaging simulation \\
Optical wavelength $(\lambda)$ & $850 \mathrm{~nm}$ \\
Altitude of the imaging system $\left(z_{0}\right)$ & $3050 \mathrm{~m}$ \\
Prop. dist. through the atmosph. $(z)$ & $15 \mathrm{~km}$ \\
Turbulence model used & Kolmogorov model \\
$C_{n}^{2}(z)$ turbulence profile & Hufnagel-Valley $(\mathrm{HV})$ \\
Number of random phase screens & 8 spaced equidistant along the opt. path \\
Number of phase screen iterations & $100-200$ iterations \\
Fried parameter $\left(r_{0}\right)$ & $10-25 \mathrm{~cm}$ \\
Space sampling in the object plane & $0.75 \mathrm{~cm}$ \\
Space sampling in the pupil plane & $0.33 \mathrm{~cm}$ \\
\hline
\end{tabular}

Table 5.1: Propagation parameters.

\subsubsection{AO system}

The parameters of the simulation concerning the AO system are presented in Table 5.3.

Figure 5.3 gives a block-diagram of the simulation.

\subsection{Sampling requirements}

In computer simulations, it is essential to respect sampling requirements [63, 64, 65]. The sampling requirements are different whether we consider the case of an optical field going through the pupil of a lens, or propagating in the atmosphere using the angular spectrum propagator. 


\begin{tabular}{|l|l|}
\hline Primary diameter & $3.67 \mathrm{~m}$ \\
Secondary diameter & $0.25 \mathrm{~m}$ \\
Spider type & quadrilateral, orthogonal \\
Width of the spider & $2 \mathrm{~cm}$ \\
Focal length $(f)$ & $726 \mathrm{~m}$ \\
Array size & $512 \times 512$ pixels \\
Field of view $(\mathrm{FOV})$ & $256 \mu \mathrm{rad}(0.5 \mu \mathrm{rad} /$ pixel $)$ \\
\hline
\end{tabular}

Table 5.2: Telescope parameters.

\begin{tabular}{|l|l|}
\hline Wavefront sensor (WFS) type & Shack-Hartmann \\
Array size & $32 \times 32$ lenslet \\
WFS detector size & $512 \times 512$ pixels \\
WFS detector wavelength $\left(\lambda_{W F S}\right)$ & $650 \mathrm{~nm}$ \\
Subaperture size & 400 microns \\
Number pixels across each subaperture & 16 pixels \\
Focal length of lenslet & 35 mm \\
Number of deformable mirror actuators & 784 actuators \\
Average number of photon events $(\bar{K})$ & $10-10^{4}$ photons/subaperture \\
\hline
\end{tabular}

Table 5.3: AO system parameters.

\subsubsection{Resolution limit}

A classical resolution limit criterion in optical systems is expressed in term of angle by

$$
\alpha_{\text {limit }}=\frac{\lambda}{D}
$$

where $D$ is the aperture size of the imaging system, and $\lambda$ the optical wavelength. The spatial resolution criterion is the projection of this angle over the propagation distance $z$ and is written

$$
\Delta x \leq \frac{\lambda z}{D}
$$




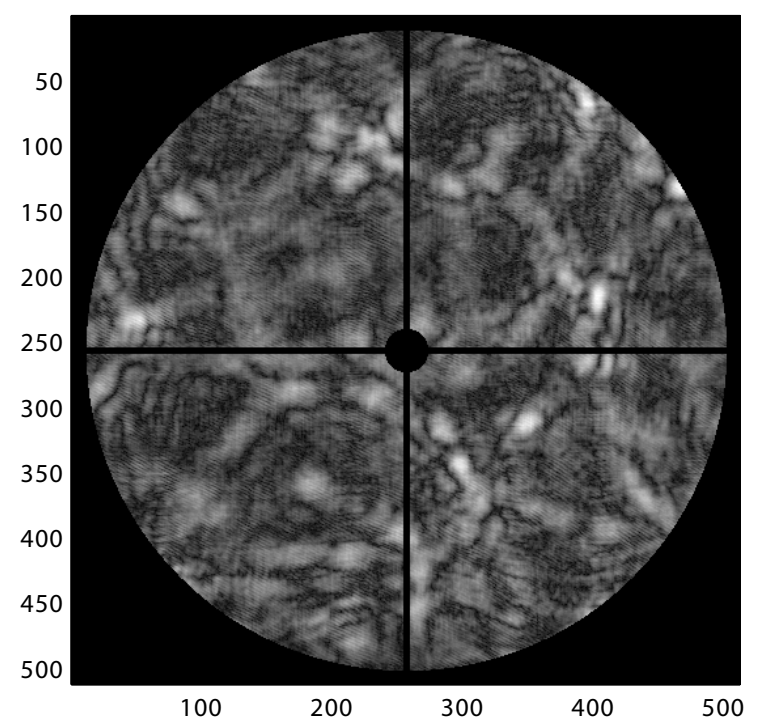

Figure 5.2: Intensity image in the telescope pupil plane.

In our simulation, we have $z=15 \mathrm{~km}, D=3.67 \mathrm{~m}$, and $\lambda=0.85 \times 10^{-9} \mathrm{~m}$. The sampling requirement in the pupil plane is then

$$
\Delta x \leq 0.3474 \mathrm{~cm},
$$

and

$$
\alpha_{\text {limit }}=0.2316 \mu \mathrm{rad} .
$$

\subsubsection{Angular spectrum propagator}

Wave propagation using an angular spectrum propagator (See section 2.3) imposes the following space sampling criterion to be respected:

$$
N \geq \frac{2 \lambda z}{\Delta x^{2}}
$$

where $\Delta x$ denotes respectively the sample spacing. $N$ is the array size, $z$ is the propagation distance through the volume of turbulence, and $\lambda$ is the optical wavelength. The reason for this requirement is to avoid the wraparound effect. The wraparound effect is an artifact of computer simulations due to the limited size $N$ of the grid used. 


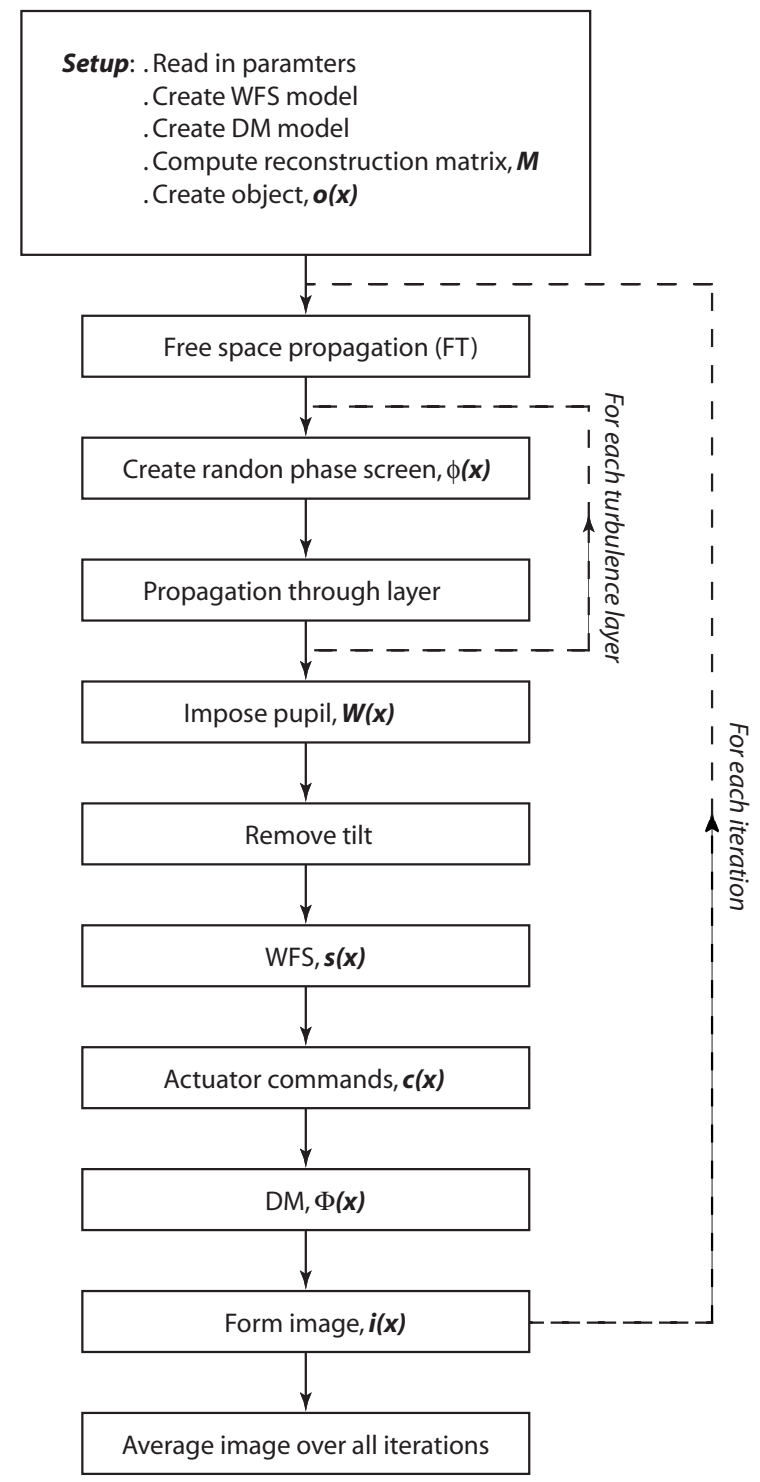

Figure 5.3: Block-diagram of the simulation. 


\subsection{Conclusion}

In this chapter, we described the wave optics simulation that we use. The details of the implementation are provided, in particular, the generation of the random phase screens. The values of the different parameters of the simulation are given, as well as the sampling requirements. 


\section{CHAPTER 6}

\section{Prediction of the off-axis point spread function}

\subsection{Introduction}

Adaptive optics systems allow the effects of turbulence-induced aberrations to be reduced for a narrow field of view corresponding approximately to the isoplanatic angle $\theta_{0}[66]$. For field angles larger than $\theta_{0}$, the PSF degrades as the field angle increases [67]. Knowledge of the space-varying PSF is essential for the reconstruction of anisoplanatic AO images. In this chapter, we present a technique to predict the long exposure (LE) AO-corrected PSF as a function of the field angle.

This chapter is organized as followed. First, we describe the approach used in the technique for prediction of the PSF. Second, by means of a wave optics simulation, we compute the LE AO-corrected PSF for different field angle values and different values of the Fried parameter $r_{0}$. The influence of the field angle $\theta$ and the Fried parameter $r_{0}$ on the PSF is discussed. In section 6.4, we study the geometrical properties of the PSF. In section 6.5, a parameterized model for the LE-PSF is presented. In section 6.6, the model of the PSF is fit to the simulated PSF. The influence of $\theta$ and $r_{0}$ on the parameters of the model is studied. In section 6.7, the PSF prediction method by interpolation is presented. section 6.8 gives the results obtained. Conclusions are drown in the last section of this chapter. 


\subsection{Approach}

The approach used to predict the average PSF as a function of the field angle is as follows. The technique assumes that we know the PSF for at least two different field angle values $\theta_{\text {known. }}$. The set composed of those field angles is denoted $\Theta_{\text {known }}$. The known PSF's are obtained by computing the image of point sources by means of an imaging simulation, as described in chapter 5. The technique allows prediction of the PSF for any field angle value $\theta_{\text {predict }}$ inside the interval $\Theta_{\text {predict }}$ defined by $\Theta_{\text {predict }}=$ $\left[\min \left\{\Theta_{\text {known }}\right\} ; \max \left\{\Theta_{\text {known }}\right\}\right]$. Figure 6.1 presents a block-diagram of the method, which consists of 3 steps:

- Step 1: The known PSF's are fit with a parameterized model of the PSF. The parameters of the model are the coefficients $a_{i}$ 's and $b_{i}$ 's. An initial guess for the coefficients $a_{i}$ and $b_{i}$ is given. Then, an optimization loop finds the coefficients $a_{i}$ and $b_{i}$ minimizing the mean square error between the known PSF and the parameterized model of the PSF. This way, we obtain a set of coefficients $a_{i}$ and $b_{i}$ for each field angle $\theta_{\text {known }} \in \Theta_{\text {known }}$.

- Step 2: By means of an interpolation method, we predict the coefficients $a_{i}$ and $b_{i}$ for the desired $\theta_{\text {predict }} \in \Theta_{\text {predict }}$.

- Step 3: Using the parameterized model, the PSF is predicted from the $a_{i}$ 's and $b_{i}$ 's for each $\theta_{\text {predict }} \in \Theta_{\text {predict }}$. 


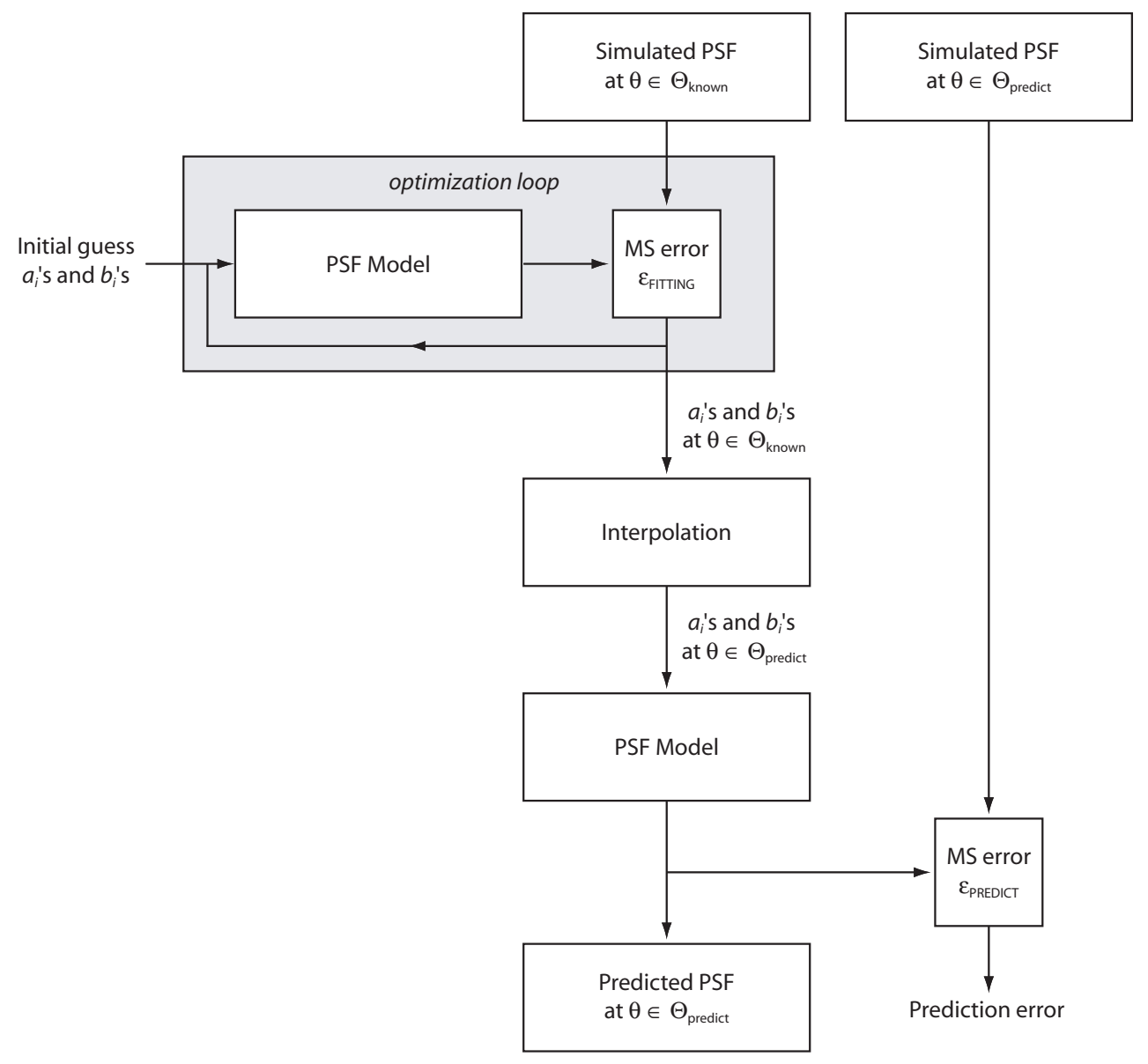

Figure 6.1: Block-diagram of the PSF prediction method. 


\subsection{Simulation of the off-axis PSF}

\subsubsection{Introduction}

The PSF is computed at field angles $\theta_{\text {known }} \in \Theta_{\text {known }}$ by mean of a wave optics simulation as described in chapter 5. We obtain the PSF for different field angle values by computing the image through turbulence for an array of point sources. The PSF is also computed for different values of the Fried parameter $r_{0}$. In this section we observe the influence of those two parameters on the PSF.

\subsubsection{Influence of the field angle on the PSF}

In this chapter, the cartesian coordinates in the image plane are denoted $\left(\theta_{x}, \theta_{y}\right)$ (angles expressed in $\mu$ radians), and the origin of the reference corresponds with the position of the reference beacon of the AO system. We compute the LE AO-corrected image of points sources distributed equidistant along the $\theta_{x}$-axis $\left(\theta_{y}=0\right)$, with $\theta_{x}$ going from 0 to $64 \mu$ radians, every $8 \mu$ radians. A total of nine PSF's is computed: one on-axis $\left(\theta_{x}, \theta_{y}\right)=(0,0)$ and eight off-axis. Figure 6.2 (a) shows a negative image of the PSF's, (b) is a log-scale representation of (a), and (c) is a cross-section of (a) along the $\theta_{x}$-axis.

We can first notice that the shape of the PSF varies with the field angle. When the field angle increases, the amplitude of the PSF decreases and the PSF becomes broader. This is a consequence of anisoplanatism on AO systems.

The field angle affects the PSF in a second manner. When the field angle gets larger, the PSF takes an elongated shape along the $\theta_{x}$-axis. This elongation is another effect of anisoplanatism on AO systems. The reason for the direction of the elongation is given by the fact that AO systems need a reference beacon. Since in our case the reference beacon is located at $\left(\theta_{x}, \theta_{y}\right)=(0,0)$, the PSF is elongated in the direction of 
the origin of the referential $\left(\theta_{x}, \theta_{y}\right)$.

\subsubsection{Influence of the Fried parameter $r_{0}$ on the PSF}

We now want to observe the influence of the Fried parameter on the PSF. The wave optics simulation is run for different $r_{0}: 10,15,20$, and $25 \mathrm{~cm}$. The intensity image obtained is averaged over 100 realizations of the phase screens. Figure 6.3 presents the PSF computed at field angle values $\theta_{x}=0,8,16,24,32,40,48,56$ and $64 \mu$ radians, for each $r_{0}$.

We can notice that the PSF at a fixed field angle varies with the Fried parameter. When $r_{0}$ decreases, the PSF gets broader and its amplitude decreases. This is due to the fact that the performance of the $\mathrm{AO}$ system decreases as the turbulence strength increases (smaller $r_{0}$ ). Furthermore, the amplitude of the PSF does not decrease at the same rate for different field angle values. The amplitude of the PSF decreases faster as the field angle increases. For example, the peak value of the on-axis $\left(\theta_{x}=0 \mu \mathrm{rad}\right)$ PSF goes from 0.55 to 0.46 when $r_{0}$ goes from 25 to $15 \mathrm{~cm}$. This represents a decrease of $16 \%$. If we consider the PSF at field angle $\theta_{x}=40 \mu \mathrm{rad}$, the peak value goes from 0.124 to 0.02 , which represents a decrease of $84 \%$.

\subsection{Geometrical properties of the PSF}

\subsubsection{Introduction}

In order to establish a simple model for the LE-PSF, we assume the PSF to respect some geometrical properties. The PSF is assumed to have rotation and symmetry properties. 

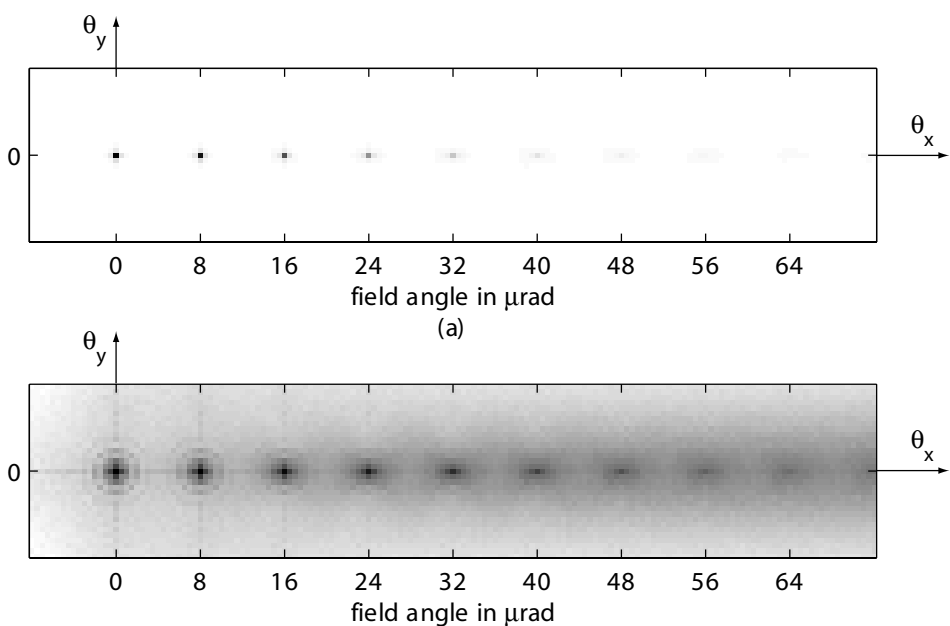

(b)

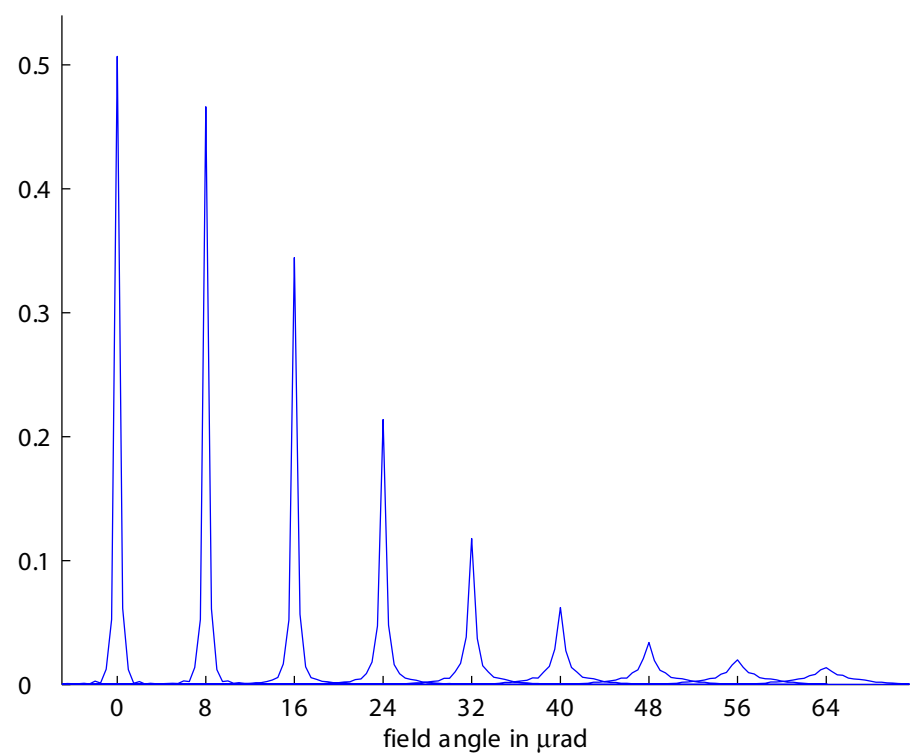

(c)

Figure 6.2: (a) LE AO-corrected PSF's computed for nine values of the field angle. (b) Log-scale representation of (b). (c) Cross-section of (a). The isoplanatic angle, which depends on the turbulence profile, is in this case $\theta_{0}=16.59$ pradians. The Fried parameter, $r_{0}$, is $20 \mathrm{~cm}$ and the number of realizations of the phase screens is 100 . 

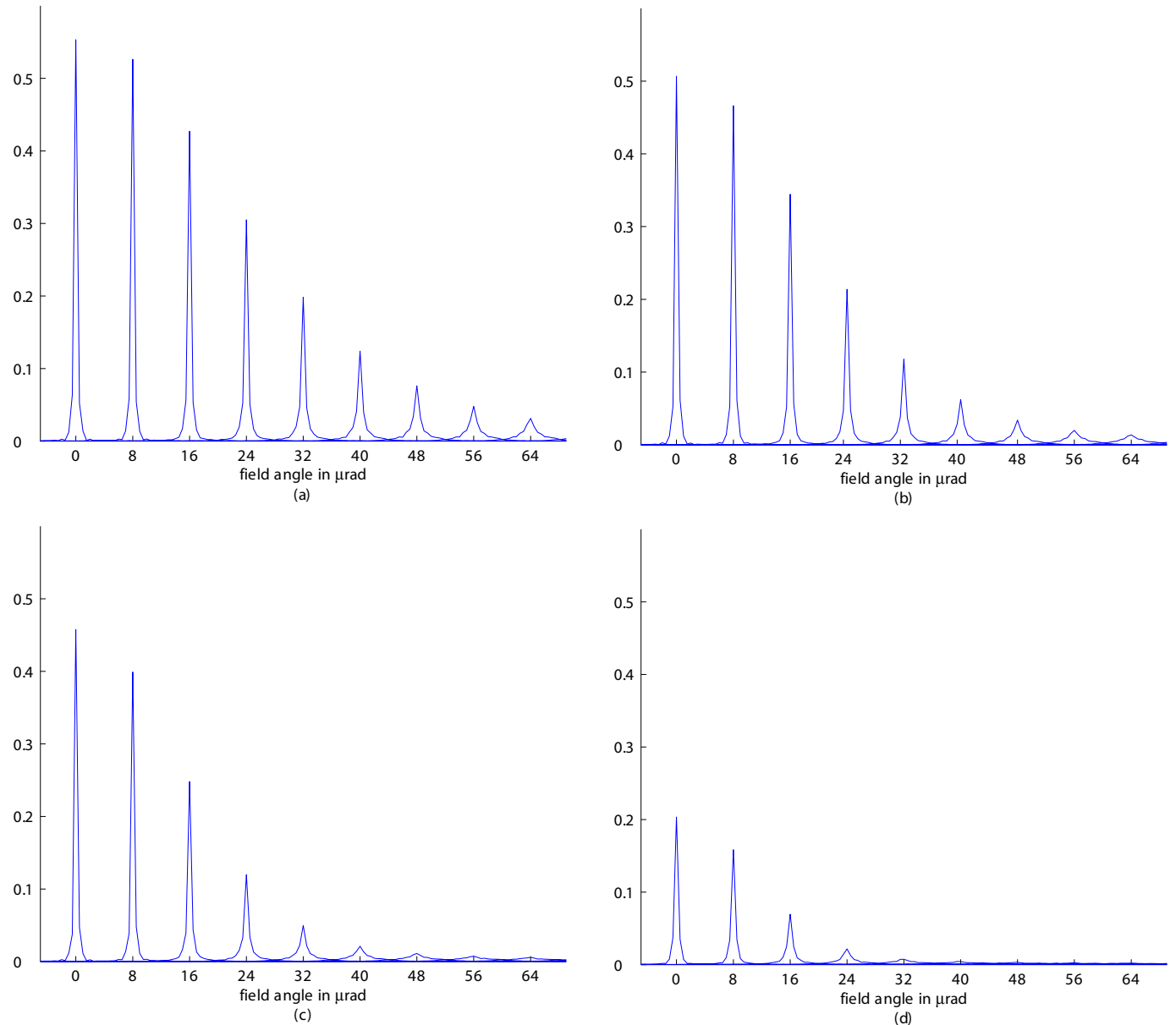

Figure 6.3: Cross-sections of the LE AO-corrected PSF computed every 8 uradians from 0 to 64 uradians for different $r_{0}:$ (a) $r_{0}=25 \mathrm{~cm}\left(\theta_{0}=20.48\right.$ uradians), (b) $r_{0}=20$ $\mathrm{cm}\left(\theta_{0}=16.59\right.$ uradians $),(c) r_{0}=15 \mathrm{~cm}\left(\theta_{0}=12.42 \mu\right.$ radians $)$, and (d) $r_{0}=10 \mathrm{~cm}$ $\left(\theta_{0}=8.3\right.$ uradians $)$. 


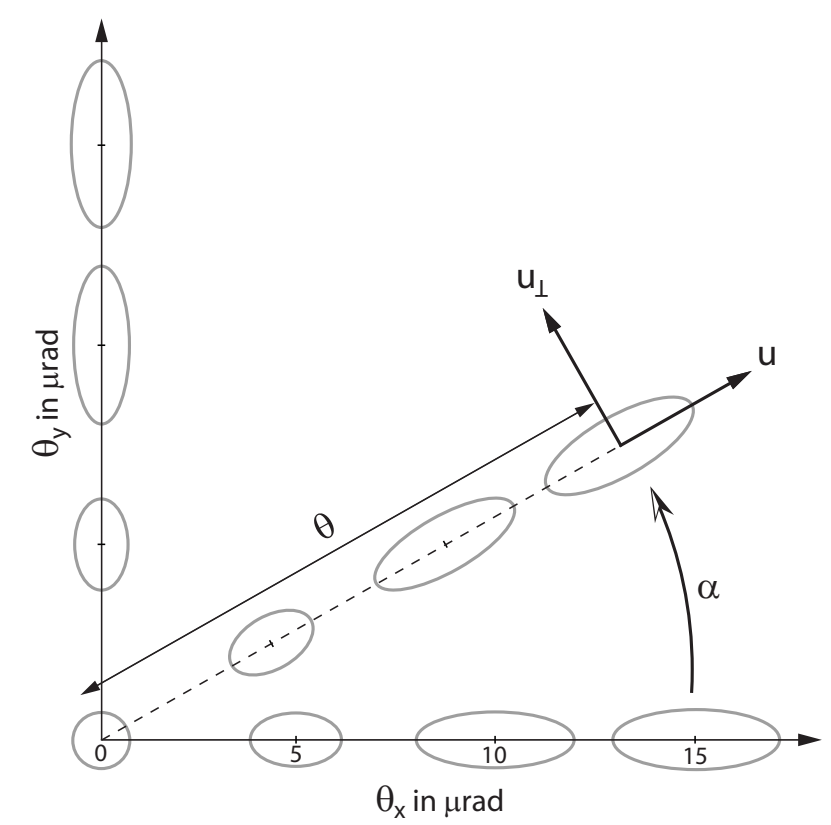

Figure 6.4: Rotation property of the PSF.

\subsubsection{Rotation property}

We assume the propagation media to be isotropic. Thus, the LE non-AO-corrected PSF has rotational symmetry property. The LE AO-corrected PSF is not rotationally symmetric, as a consequence of anisoplanatism. However, the LE PSF respect a property of rotation as showed in Figure 6.4. In Figure 6.4, the position of the center of the PSF is given by the polar coordinates $(\theta, \alpha)$. The radial component $\theta$ is defined by $\theta=\sqrt{\theta_{x}^{2}+\theta_{y}^{2}}$ (in $\mu$ radians) and corresponds to the angular separation between the reference beacon and the center of the PSF (also known as field angle). The variable $\alpha$ is the angular component of the polar coordinates and corresponds to the orientation of the PSF in the image plane. The angle $\alpha$ is expressed in degrees.

We introduce a new reference $\left(u, u_{\perp}\right)$, as showed in Figure 6.4. The reference $\left(u, u_{\perp}\right)$ is defined such that its origin corresponds to the center of the PSF. The PSF can be now expressed in term of the spatial variables $u$ and $u_{\perp}$. Since the PSF is space-varying, its expression depends also on the field angle $\theta$ and the orientation $\alpha$. Thus, in its most 
general form, the PSF is denoted $h_{\theta, \alpha}\left(u, u_{\perp}\right)$.

The first geometrical approximation for the model of the PSF is a rotation property. As a consequence, the PSF does not depend on the orientation of the PSF $\alpha$, but only on the field angle value $\theta$. For example, the PSF at $\theta=10 \mu$ rad and $\alpha=0$ degree is the same than the PSF at $\theta=10 \mu \mathrm{rad}$ and $\alpha=90$ degrees, rotated of 90 degrees. Only the orientation of the PSF in the reference $\left(\theta_{x}, \theta_{y}\right)$ changes. Therefore, the PSF can be denoted $h_{\theta}\left(u, u_{\perp}\right)$.

The rotation property of the PSF around the origin of the reference is due to the fact that the reference beacon of the $\mathrm{AO}$ system is positioned at the origin.

Note: The rotation property approximation presents a very convenient aspect: from the knowledge of the PSF along the $\theta_{x}$-axis, we can deduce the PSF in the entire image plane by rotation $\alpha$ around the origin.

\subsubsection{Symmetry property}

We assume the PSF to be symmetrical with respect to the $u$-axis and the $u_{\perp}$-axis, as showed by Figure 6.5. Lets now consider the cross-sections of the PSF $h_{\theta}\left(u, u_{\perp}\right)$ through the $u$-axis and the $u_{\perp}$-axis. Those cross-sections are denoted $h_{\theta ; u}(u)$ and $h_{\theta ; u_{\perp}}\left(u_{\perp}\right)$. As an example, Figure 6.6 represents the cross-sections of the PSF at $\theta=$ $24 \mu$ radians, and for $r_{0}=15 \mathrm{~cm}$.

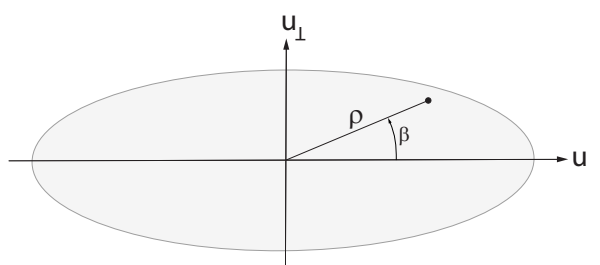

Figure 6.5: Symmetry property of the PSF. 


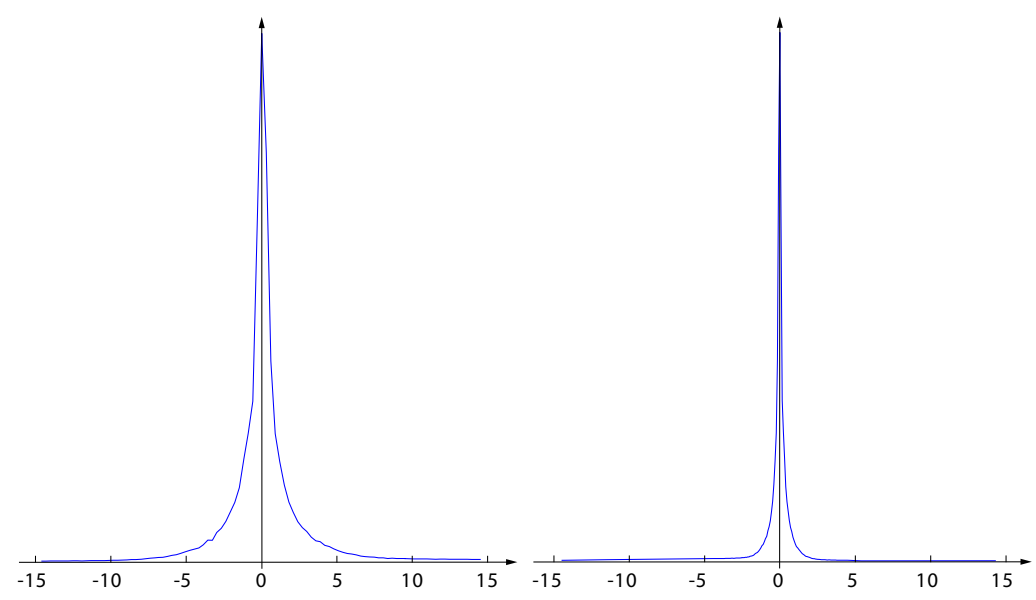

(a)

(b)

Figure 6.6: Cross-sections of the simulated PSF at $\theta=24 \mu$ radians and for $r_{0}=15$ $c m:$ (a) $h_{\theta ; u}(u)$ and (b) $h_{\theta ; u_{\perp}}\left(u_{\perp}\right)$.

\subsection{Parameterized model of the PSF}

\subsubsection{Model}

The two-dimensional PSF $h_{\theta}\left(u, u_{\perp}\right)$ is now parameterized as a function of its two one-dimensional cross-section functions $h_{\theta ; u}(u)$ and $h_{\theta ; u_{\perp}}\left(u_{\perp}\right)$. Expressed in polar coordinates, the parameterized model is

$$
h_{\theta}(\rho, \beta)=(1-\gamma(\beta)) h_{\theta ; u}(\rho)+\gamma(\beta) h_{\theta ; u_{\perp}}(\rho),
$$

where $(\rho, \beta)$ are the polar coordinates as defined in Figure 6.5. The function $\gamma(\beta)$ is given by

$$
\gamma(\beta)=|1-| \frac{\beta}{\frac{\pi}{2}}||,
$$

for $-\pi<\beta<\pi$.

The function $\gamma(\beta)$ is chosen such that for a fixed $\rho$ (radial component), $h_{\theta}(\rho, \beta)$ follows a linear progression between the cross-sections $h_{\theta ; u}(u)$ and $h_{\theta ; u_{\perp}}\left(u_{\perp}\right)$ inside each quadrant of the reference $\left(u, u_{\perp}\right)$. For example, in the first quadrant $(0<\beta<\pi / 2)$, 
the function $\gamma(\beta)$ becomes

$$
\gamma(\beta)=1-\frac{\beta}{\frac{\pi}{2}}
$$

Thus, $\gamma(\beta)$ varies linearly from 1 to 0 , allowing $h_{\theta}(\rho, \beta)$ to vary linearly from $h_{\theta ; u}(u)$ to $h_{\theta ; u_{\perp}}\left(u_{\perp}\right)$, for a fixed $\rho$. Figure 6.7 (a) is a plot of $\gamma(\beta)$ for $-\pi<\beta<\pi$ and (b) is a 3 -D plot of $\gamma(\beta)$ in the referential $\left(\theta_{x}, \theta_{y}\right)$. Now that we established a relationship
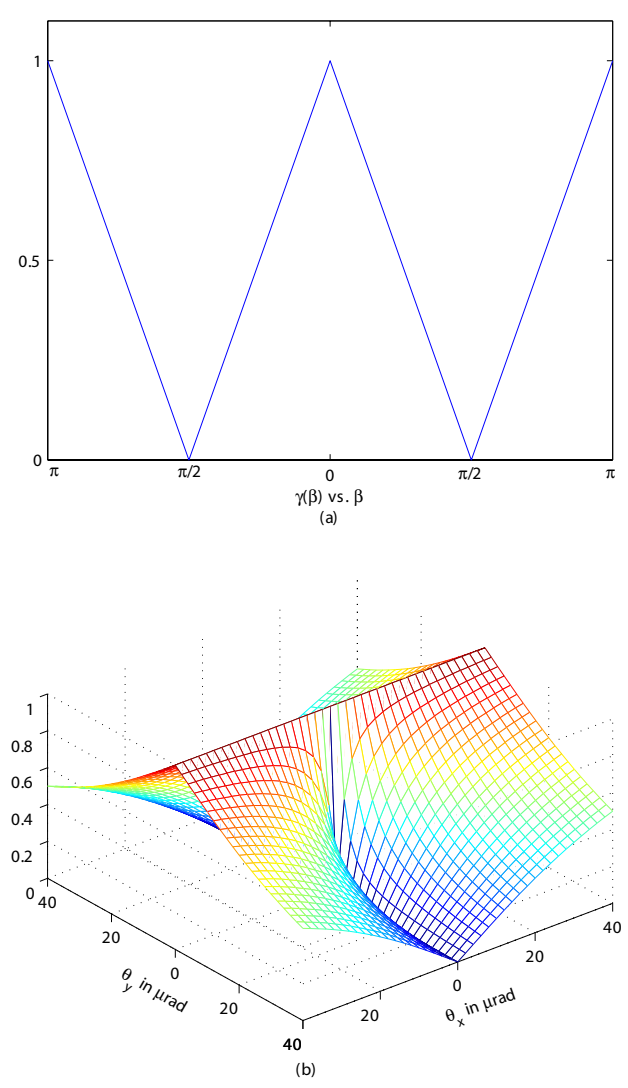

Figure 6.7: (a) Plot of the function $\gamma(\beta)$. (b) 3-D plot of $\gamma(\beta)$ in cartesian coordinates. between the PSF ant its cross-sections, we want to find a model for the cross-section in order to complete our model of the PSF. 


\subsubsection{Model for the PSF cross-section}

We want to fit a mathematical function to the simulated cross-sections $h_{\theta ; u}(u)$ and $h_{\theta ; u_{\perp}}\left(u_{\perp}\right)$. We consider only the case of the cross-section along the $u$-axis, $h_{\theta ; u}(u)$, since $h_{\theta ; u_{\perp}}\left(u_{\perp}\right)$ is fit using the same method. Due to the symmetry properties of the PSF (See section 6.4), the cross-section $h_{\theta ; u}(u)$ is also symmetrical. For this reason, we only consider $h_{\theta ; u}(u)$ for $u \geq 0$.

Several mathematical functions $h_{\theta ; u} \operatorname{MODEL}(u)$ were considered: Gaussian, superGaussian, polynomial, and Chebyshev polynomial functions. We test their ability to fit the cross-section data in term of the mean square (MS) error metric, $\epsilon_{M O D E L}$, defined by

$$
\epsilon_{M O D E L}^{2}(\theta)=100 \times \frac{\sum_{u}\left\|h_{\theta ; u} \operatorname{MODEL}(u)-h_{\theta ; u}(u)\right\|^{2}}{\sum_{u}\left\|h_{\theta ; u}(u)\right\|^{2}} .
$$

It is interesting to note that $\epsilon_{M O D E L}$ is a function of the field angle $\theta$. The choice of the fitting function is empirical. The mathematical function providing the smallest $\epsilon_{M O D E L}$ will be used.

\subsubsection{Mathematical functions}

\section{- Gaussian function}

The first mathematical function considered is the Gaussian function, defined by

$$
h_{G}(u)=A \exp \left\{-\frac{u^{2}}{\sigma^{2}}\right\},
$$

where $A$ characterizes the amplitude of the Gaussian and $\sigma$ its $e^{-1}$ radius. The Gaussian function is convenient because it has only two parameters.

\section{- Super-Gaussian function}

The second mathematical function considered is a modified version of the zeromean Gaussian, called super-Gaussian. The zero-mean super-Gaussian is defined 
by

$$
h_{S G}(u)=\exp \left\{-\sum_{i=0}^{N} a_{i} u^{2 i}\right\},
$$

where $N$ denotes the order of the super-Gaussian.

\section{- Polynomial function}

We also consider the polynomial function defined by

$$
h_{P O L Y}(u)=\sum_{i=0}^{N} a_{i} u^{i},
$$

where $N$ is the order of the polynomial.

\section{- Chebyshev polynomial function}

The Chebyshev polynomial of the first kind, $T_{n}(u)$, is defined by

$$
T_{n}(u)=\cos (n \arccos (u)),
$$

where $n$ is the order of the polynomial. The Chebyshev polynomials are considered because they present the interesting property of being orthogonal on the interval $[-1 ; 1]$. The advantage of orthogonal functions is that a signal has a unique decomposition as a serie of those functions. Chebyshev polynomials can be recursively calculated using the following relationship:

$$
T_{n}(u)=2 T_{n-1}^{2}(u)-T_{n-2}(u),
$$

with

$$
T_{0}(u)=1,
$$

and

$$
T_{1}(u)=u \text {. }
$$

Table 6.1 shows the first seven Chebyshev polynomials. Using the Chebyshev polynomials, we define the following fitting function:

$$
h_{C H E B Y}(u)=\sum_{i=0}^{N} a_{i} T_{i}(u) .
$$


The parameter $N$ is the order of the Chebyshev polynomial function.

\begin{tabular}{|c|l|}
\hline Order & Chebyshev polynomial \\
\hline 0 & 1 \\
1 & $u$ \\
2 & $2 u-1$ \\
3 & $4 u^{3}-3 u$ \\
4 & $8 u^{4}-8 u^{2}+1$ \\
5 & $16 u^{5}-20 u^{3}+5 u$ \\
6 & $32 u^{6}-48 u^{4}+18 u^{2}-1$ \\
\hline
\end{tabular}

Table 6.1: First seven Chebyshev polynomials of the first kind.

\subsubsection{Comparison of the models for the cross-sections}

We consider the fitting of $h_{\theta ; u} M O D E L(u)$ to the data $h_{\theta ; u}(u)$ for different field angle values: $\theta=0 \mu \mathrm{rad}$ (on-axis) and $\theta=32 \mu \mathrm{rad}$ (off-axis). Each set of data is fit with the four mathematical functions previously introduced. For the super-Gaussian function, the polynomial function and the Chebyshev polynomial function, we consider different orders. Figure 6.8 is a plot of the relative MS error as a function of the order of the fitting function; expect the Gaussian function, which has only two parameters.

From Figure 6.8, we can notice different properties of the fitting functions. First, the polynomial function is not appropriate for the fitting of the PSF since the error is always greater than $20 \%$ and the error increases when the order of the polynomial is greater than 5. Second, we can notice that the super-Gaussian fit converges very fast. However, even for high order, the fitting error does not decrease. The Chebyshev polynomial is a more stable solution for high orders than the polynomial function. Figure 6.9 shows the cross-section of the PSF and the fitting functions. 

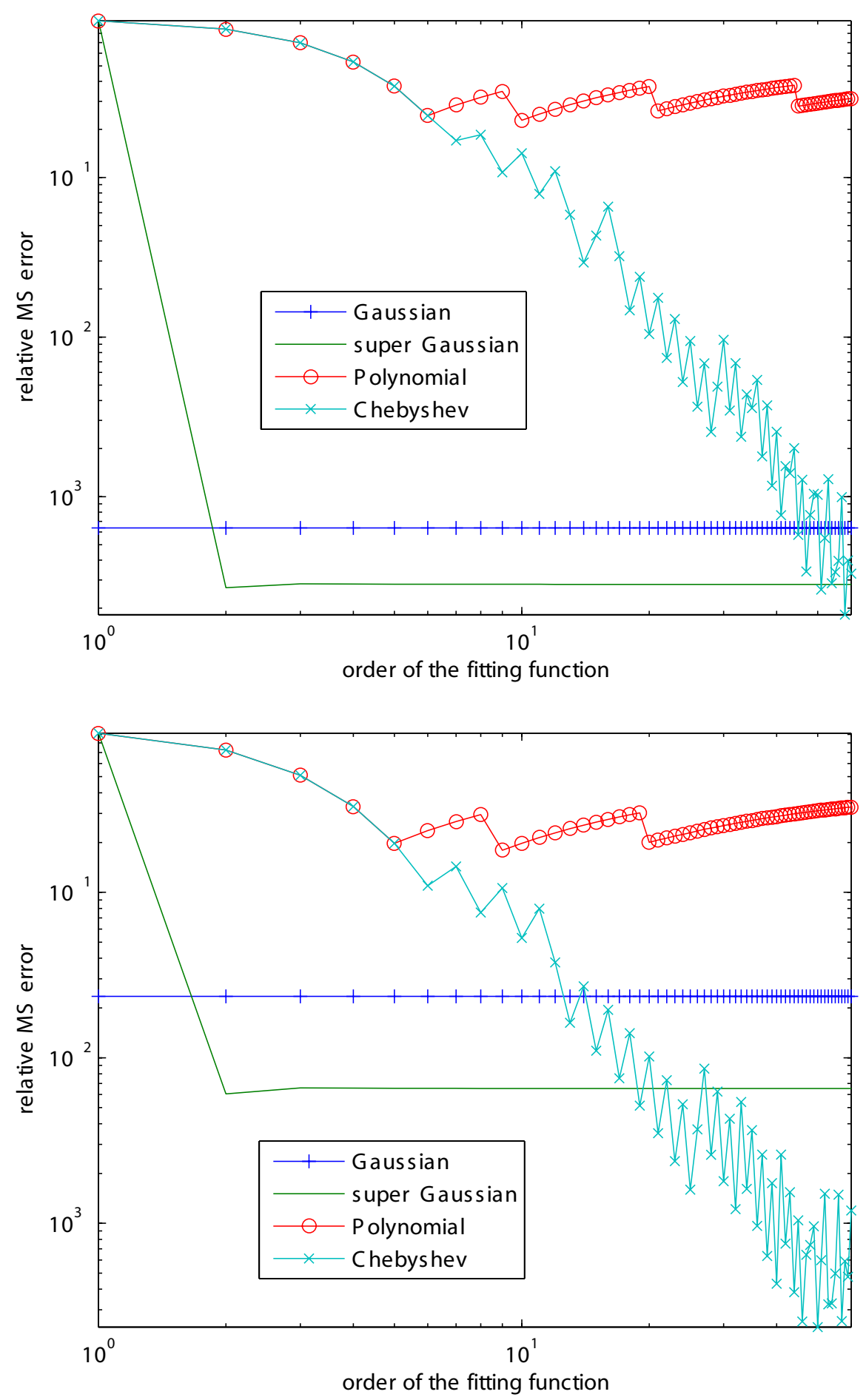

Figure 6.8: Relative $M S$ error $\epsilon_{M O D E L}$ vs. order $N$ of the fitting function. The PSF is computed for a Fried parameter is $r_{0}=20 \mathrm{~cm}$ and a field angle of (a) $\theta=0$ rrad and (b) $\theta=32 \mu \mathrm{rad}$. 
Tables 6.2 and 6.3 give the smallest relative error obtained for each fitting function and for different orders.

\begin{tabular}{|r|c|c|}
\hline Fitting function & 30th Order & 60th Order \\
\hline Gaussian & \multicolumn{2}{|c|}{0.63} \\
Super-Gaussian & $\mathbf{0 . 2 2}$ & 0.22 \\
Polynomial & 22.3 & 22.3 \\
Chebyshev & 0.33 & $\mathbf{0 . 1 7}$ \\
\hline
\end{tabular}

Table 6.2: Relative MS error (\%) for fitting the on-axis PSF $(\theta=0 \mu \mathrm{rad})$.

\begin{tabular}{|r|c|c|}
\hline Fitting function & 30th Order & 60 th Order \\
\hline Gaussian & \multicolumn{2}{|c|}{2.68} \\
Super-Gaussian & 0.58 & 0.58 \\
Polynomial & 16.7 & 16.7 \\
Chebyshev & $\mathbf{0 . 1 9}$ & $\mathbf{0 . 0 1 2}$ \\
\hline
\end{tabular}

Table 6.3: Relative MS error (\%) for fitting an off-axis PSF, at $\theta=32 \mu \mathrm{rad}$.

In Tables 6.2 and 6.3, we can notice that the fit does not improve with the order in the case of a polynomial or super-Gaussian function. However, in the case of the Chebyshev polynomial, the error gets smaller when the order becomes greater. For this reason, we choose the Chebyshev polynomial function as a model for the cross-sections. Thus, the PSF given by Eq. 6.1 is modelized by $h_{\theta} \operatorname{MODEL}(\rho, \beta)$, which can be written

$$
h_{\theta \operatorname{MODEL}}(\rho, \beta)=\gamma(\beta) h_{\theta ; u_{\perp} M O D E L}(\rho)+(1-\gamma(\beta)) h_{\theta ; u} \operatorname{MODEL}(\rho),
$$

with

$$
h_{\theta, u_{\perp}} \operatorname{MODEL}\left(u_{\perp}\right)=\sum_{i=0}^{N} a_{i}(\theta) T_{i}\left(u_{\perp}\right)
$$

and

$$
h_{\theta, u} \operatorname{MODEL}(u)=\sum_{i=0}^{N} b_{i}(\theta) T_{i}(u) \text {, }
$$




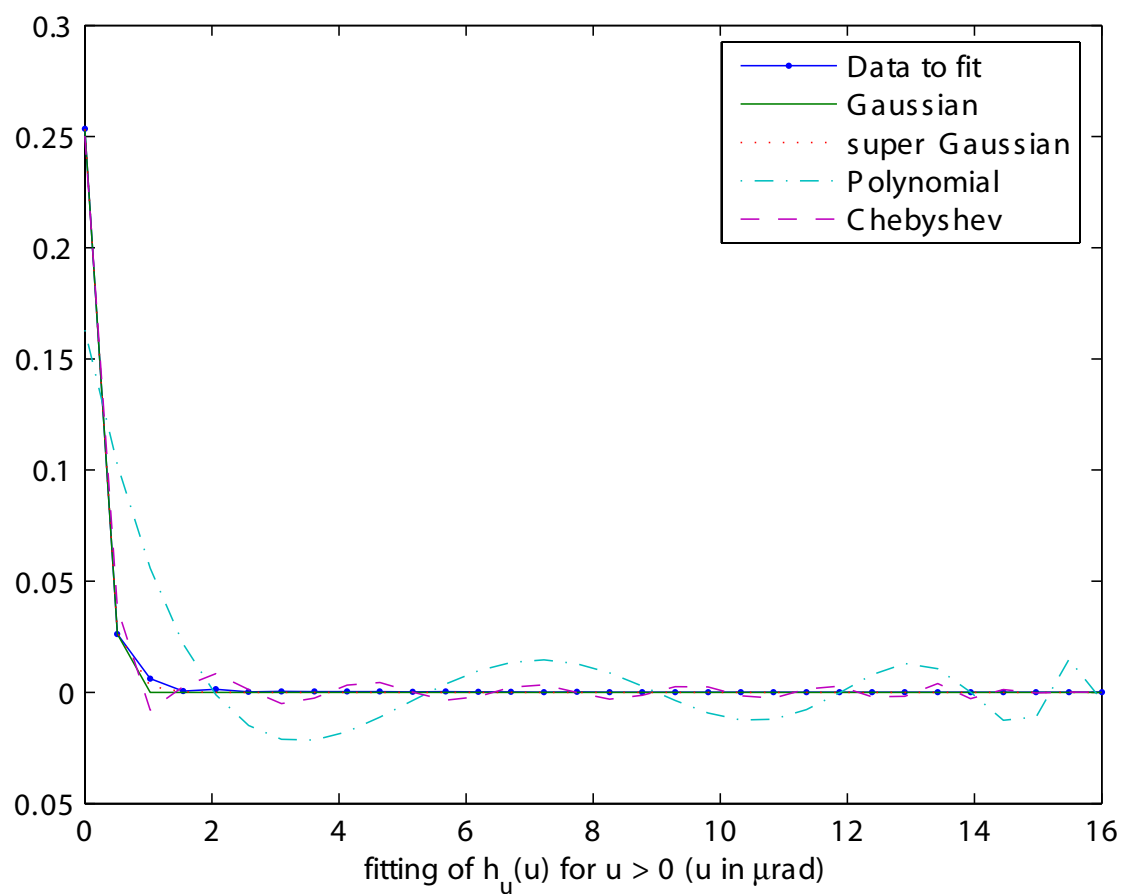

(a)

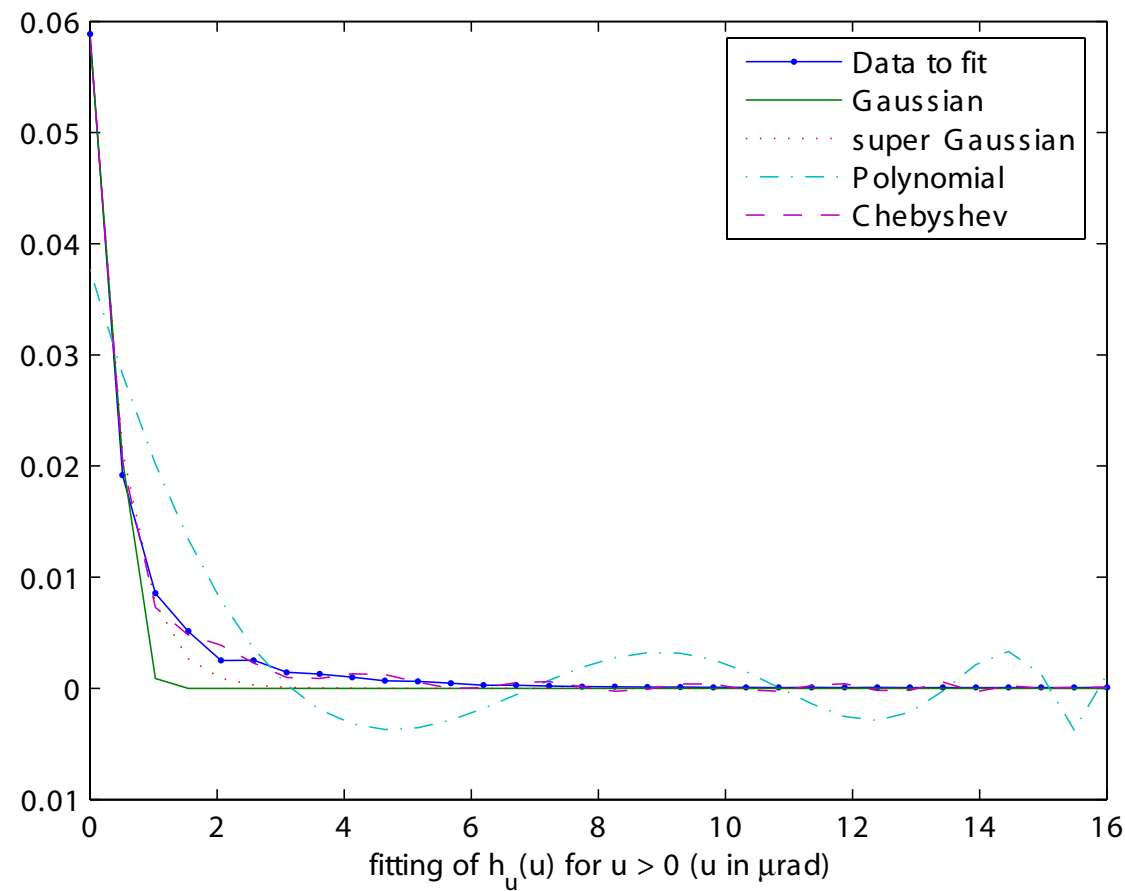

(b)

Figure 6.9: Fitting of the cross-section $h_{\theta ; u}(u)$ for $u \geq 0$ with different fitting functions of 30th order. The PSF is computed for a Fried parameter is $r_{0}=20 \mathrm{~cm}$ and a field angle of (a) $\theta=0 \mu \mathrm{rad}$ and (b) $\theta=32 \mu \mathrm{rad}$. 
where $N$ is the order of the Chebyshev polynomial function. Thus, we have established a parameterized model for the PSF. The parameters of the model are the $a_{i}$ 's and $b_{i}$ 's for $i \in[0, N]$.

\subsection{Fitting the model of the PSF to the simulated PSF}

\subsubsection{Fitting error}

In this section, we fit the model for the PSF established in section 6.5 to the PSF we simulated for $\theta \in \Theta_{\text {known. }}$. This way, we get the coefficients $a_{i}$ and $b_{i}$ for $\theta \in \Theta_{\text {known }}$. The fit minimizes the MS error metric $\epsilon_{\text {FITTING }}$ defined by

$$
\epsilon_{\text {FITTING }}^{2}=100 \times \frac{\sum_{u} \sum_{u_{\perp}}\left\|h_{\theta M O D E L}\left(u, u_{\perp}\right)-h_{\theta}\left(u, u_{\perp}\right)\right\|^{2}}{\sum_{u} \sum_{u_{\perp}}\left\|h_{\theta}\left(u, u_{\perp}\right)\right\|^{2}},
$$

for $\theta \in \Theta_{\text {known }}$. It is important to understand that $\epsilon_{\text {FITTING }}$ gives a measurement of a two-dimensional fit of the entire PSF, as opposed to $\epsilon_{M O D E L}$ (see Eq. 6.5) which gives a measurement of a one-dimensional fit of the cross-section. The optimization of the $a_{i}$ 's and the $b_{i}$ 's to minimize $\epsilon_{F I T T I N G}$ is implemented in MATLAB with the function fminunc.

Note: As we saw in section 6.5.4, the error $\epsilon_{\text {FITTING }}$ generally decreases as $N$ becomes greater. Thus, to obtain a desired fitting error, we simply need to increase the order $N$. In our simulations, we choose $N$ arbitrarily such that

$$
\epsilon_{F I T T I N G} \leq 1 \%
$$

Results concerning the fit of the model of the PSF to the simulated PSF are presented in Appendix A. Figure 1.1 is a plot of the fitting errors as a function of the field angle for different $r_{0}$. 
First, we can notice that the fitting error $\epsilon_{\text {FITTING }}$ is field angle dependent. The error $\epsilon_{\text {FITTING }}$ gets smaller when the field angle increases. This can be explained by the fact that the PSF gets broader and smoother as the field angle increases. Therefore, the PSF contains less high spatial frequencies, which is easier to fit with Chebyshev polynomials.

Second, we can note that if we the order $N$ equals $50, \epsilon_{\text {FITTING }}$ is always smaller than $1 \%$.

Figures 1.2 to 1.5 (Appendix A) present the two-dimensional plots of the simulated and parameterized PSF's for $\theta=0,16$, and $32 \mu$ radians, and for different $r_{0}$. First we notice that the parameterized PSF has the same general shape than the simulated PSF. Second, we also notice a ringing effect in some parameterized PSF's. For example, it is the case in Figure 1.4, for the parameterized PSF at $\theta=32 \mu$ radians. This effect is due to the oscillating nature of the Chebyshev polynomials. This is explained when looking at the definition of the Chebyshev polynomials in term of the cosine functions, as expressed in Eq. 6.8.

\subsubsection{Influence of the field angle on the parameters of the PSF model}

Figures 1.6 to 1.9 (Appendix A) present the first seven coefficients $a_{i}$ 's and $b_{i}$ 's of the PSF model as a function of the field angle $\theta$, for different $r_{0}$. Each coefficient $a_{i}$ or $b_{i}$ tends to zero when the field angle increases. This can be explained by the fact that the amplitude of the PSF decreases when the field angle increases.

The on-axis $\operatorname{PSF}(\theta=0)$ follows the rotational symmetry property. Thus, the two cross-sections of the PSF are identical and $a_{i}(\theta=0)=b_{i}(\theta=0)$, for $i \in[0 ; N]$. For $\theta>0$, the coefficients $a_{i}$ and $b_{i}$ are not identical, but they present similar features. We 
saw in section 6.3.2 that the PSF has a more elongated appearance along the $u$-axis than the $u_{\perp}$-axis. As a consequence, the cross-sections $h_{\theta ; u_{\perp}}\left(u_{\perp}\right)$ and $h_{\theta ; u}(u)$ have similarities, but for $\theta>0 h_{\theta ; u}(u)$ has is more elongated than $h_{\theta ; u_{\perp}}\left(u_{\perp}\right)$. This explains the fact that the $a_{i}$ 's and $b_{i}$ 's have the same features, with different amplitudes. Since the coefficients $a_{i}$ and $b_{i}$ correspond respectively to the cross-sections $h_{\theta ; u_{\perp}}\left(u_{\perp}\right)$ and $h_{\theta ; u}(u)$, the $a_{i}$ 's present smaller amplitudes than the $b_{i}$ 's.

We want to quantify the elongation of the PSF. Since the coefficients $b_{i}$ present the same features than the $a_{i}$ 's with smaller amplitudes, we define the elongation factor $\chi$ as the average of the ratio $b_{i} / a_{i}$. The elongation factor is field angle dependent and is given by

$$
\chi(\theta)=\frac{1}{N+1} \sum_{i} \frac{b_{i}}{a_{i}} .
$$

Figure 1.10 (Appendix A) is a plot of the elongation factor as a function of the field angle. We can note that overall $\chi$ decreases with the field angle. However, for large $\theta$ and small $r_{0}$, the elongation factor does not decrease anymore and even increases to values larger than 1 . This can be explained by the fact that for large $\theta$ and small $r_{0}$ the amplitude of the PSF becomes very small and noisy. Therefore, the fit with model of the PSF is less accurate and so are the coefficients $a_{i}$ and $b_{i}$.

\subsubsection{Influence of $r_{0}$ on the parameters of the PSF model}

Figure 6.10, and Figures 1.11 and 1.12 in Appendix A, present the first coefficients $a_{i}$ 's and $b_{i}$ 's of the PSF model as a function of $r_{0}$, for different $\theta$.

The first property to notice is that for a fixed field angle $\theta$, the plots of $a_{i}\left(r_{0}\right)$ and $b_{i}\left(r_{0}\right)$ have the same general features. As the turbulence strength decreases (larger $\left.r_{0}\right)$, the coefficients increase. This is explained by the fact that the PSF amplitude becomes larger for a less turbulent atmosphere. 
However, the coefficients do not increase at the same rate for different field angles. For the on-axis PSF, Figure 6.10 shows that for $r_{0}$ larger than $15 \mathrm{~cm}$, the coefficients $a_{i}$ and $b_{i}$ are almost constant. This means that the on-axis PSF is almost invariant with strength of the turbulence, which demonstrates that $\mathrm{AO}$ systems give their best results around the reference beacon. On the other hand, for larger field angles (see Figure 1.12 in Appendix A) the coefficients grow with $r_{0}$.

\subsubsection{Influence of the wavefront sensor noise on the para- meters of the PSF model}

In chapter 4, we established that the measurement error in a Shack-Hartman wavefront sensor (WFS) depends on several parameters. In particular it depends on the total average detected photon count per subaperture of the WFS, denoted $\bar{K}$. We now study the influence of $\bar{K}$ on the coefficients of the model $a_{i}$ and $b_{i}$. Figure 6.11, and Figures 1.13 and 1.14 in Appendix A, give plots of the $a_{i}$ 's and $b_{i}$ 's as a functions of $\bar{K}$, for different field angles. For each figure, the Fried parameter is $r_{0}=20 \mathrm{~cm}$.

In those figures, we can notice the coefficients $a_{i}$ and $b_{i}$ increase with $\bar{K}$. As the number of photon events per subaperture in the WFS increases, the WFS gets smaller and the PSF becomes more narrow and its amplitude increases. This explains the increase of the amplitude of the coefficients.

We can see that all the coefficients increase with a similar rate, except for coefficients $b_{i}$ 's at $\theta=32 \mu \mathrm{rad}$ (Figure 1.14 in Appendix A). The unexpected growth rate of the $b_{i}$ 's compared to other cases can be interpreted by a bad fit of the model of the PSF to the data. The bad fit of the model can be caused by photon noise in the PSF, but also by the finite number of iterations used in the Monte Carlo simulation (in our case 100 iterations are used), which makes the simulated data more noisy. Ideally, we could 

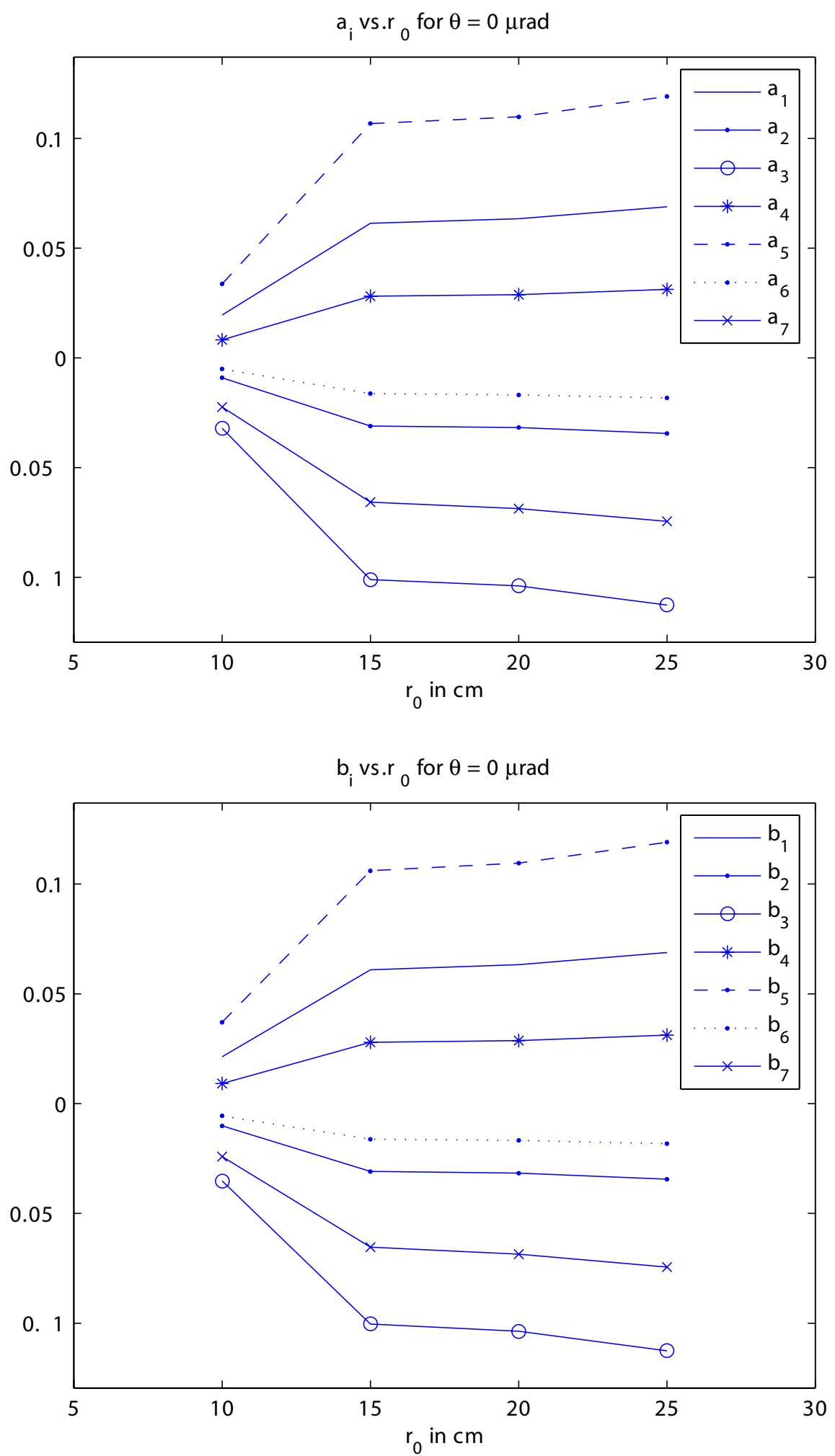

Figure 6.10: Coefficients $a_{i}$ 's and $b_{i}$ 's vs. $r_{0}$ for $\theta=0 \mu \mathrm{rad}$. 
reduce this source of noise by running the simulation for many more realizations of the random phase screens, but we are limited by the computational power of computers.

\subsection{PSF prediction by interpolation}

\subsubsection{Interpolation in term of the field angle}

Now that we know the coefficients $a_{i}$ and $b_{i}$ for each $\theta_{\text {known }} \in \Theta_{\text {known }}$, we interpolate them to predict the coefficients for $\theta_{\text {predict }} \in \Theta_{\text {predict }}$. In our simulations, we $\Theta_{\text {known }}=$ $[0,8,16,24,32] \mu$ radians and $\Theta_{\text {predict }}=[4,12,20,28] \mu$ radians. Several interpolation methods had been tested. The linear interpolation gave the best results. After we obtain the $a_{i}$ and $b_{i}$ by interpolation, we can predict the PSF for $\theta_{\text {predict }} \in \Theta_{\text {predict }}$.

Figures 1.15 to 1.18 (Appendix A) show the predicted and the simulated PSF for $\theta_{\text {predict }} \in \Theta_{\text {predict }}$, for different $r_{0}$. Figures 1.19 and 1.20 are cross-sections of the predicted PSF.

The simulated and predicted PSF's have the same general shapes. The crosssections show that each predicted PSF has the same aspect than the simulated PSF within a smaller angular separation. This is expected, since it is reasonable to anticipate that the PSF is a continuous function of the field angle.

\subsection{Results}

To measure the quality of the predicted PSF at a field angles $\theta_{\text {predict }} \in \Theta_{\text {predict }}$, we define the prediction error metric $\epsilon_{P R E D I C T}^{2}$ by

$$
\epsilon_{P R E D I C T}^{2}=\frac{\sum_{u} \sum_{u_{\perp}}\left\|h_{\theta P R E D I C T}\left(u, u_{\perp}\right)-h_{\theta}\left(u, u_{\perp}\right)\right\|^{2}}{\sum_{u} \sum_{u_{\perp}}\left\|h_{\theta}\left(u, u_{\perp}\right)\right\|^{2}},
$$




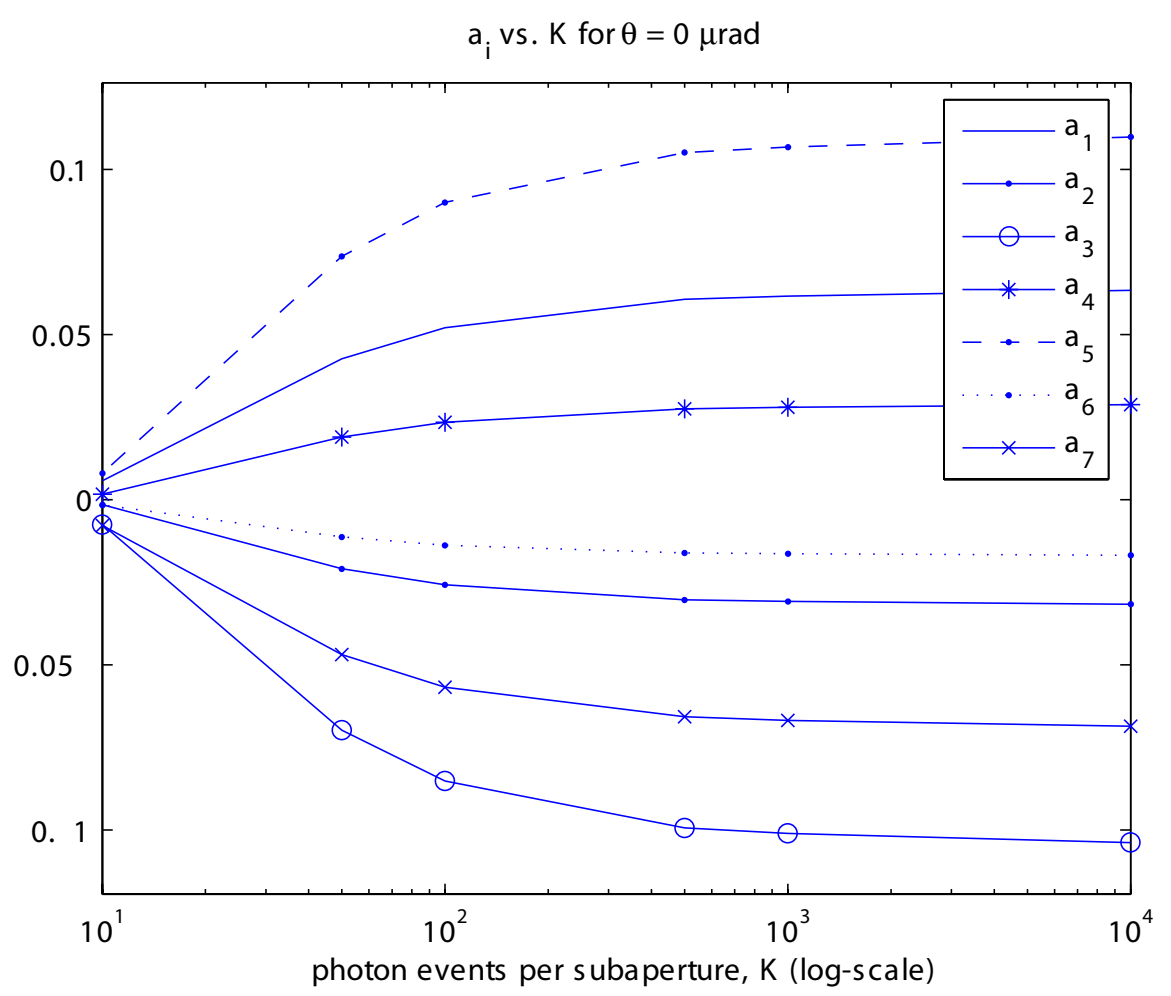

$b_{i}$ vs. $K$ for $\theta=0 \mu$ rad

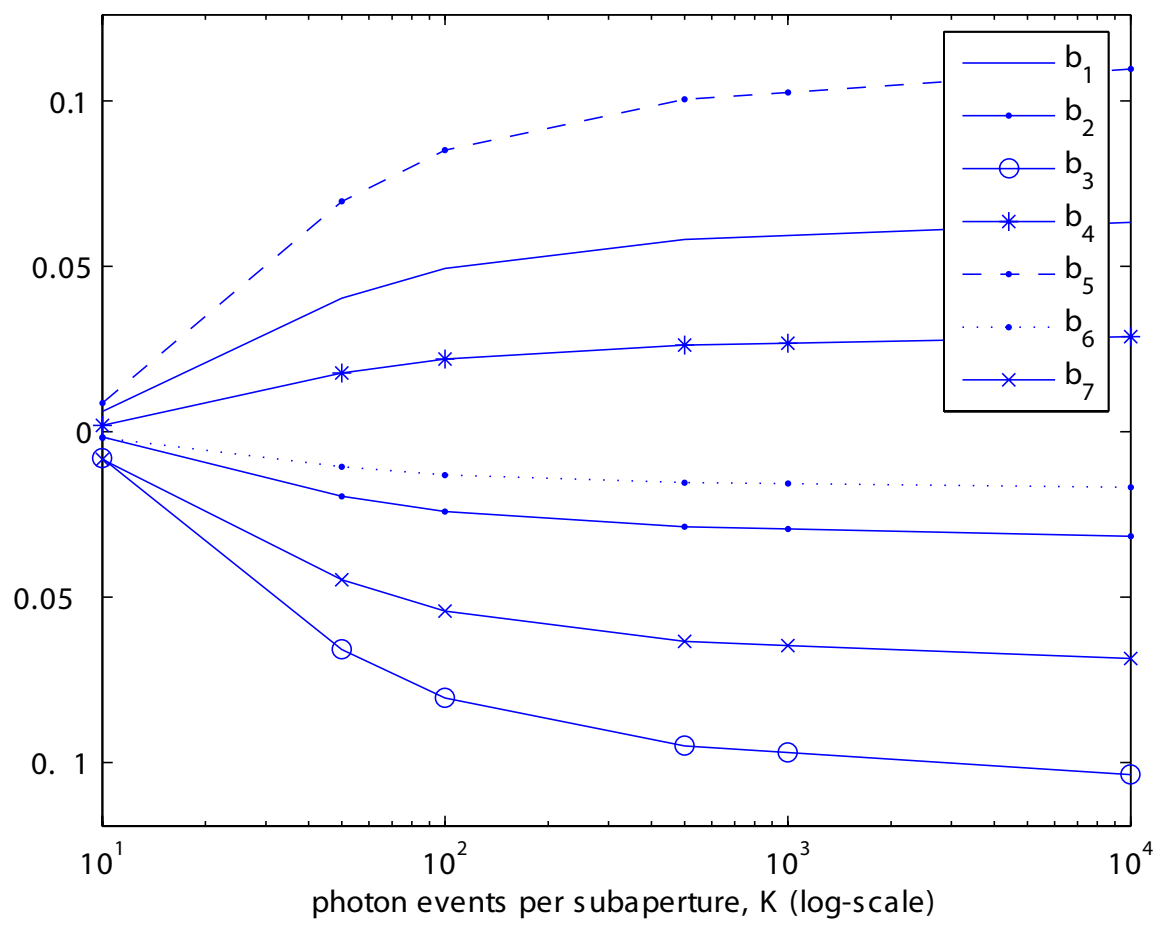

Figure 6.11: Coefficients $a_{i}$ 's and $b_{i}$ 's vs. $\bar{K}$ for $\theta=0 \mu r a d$. 


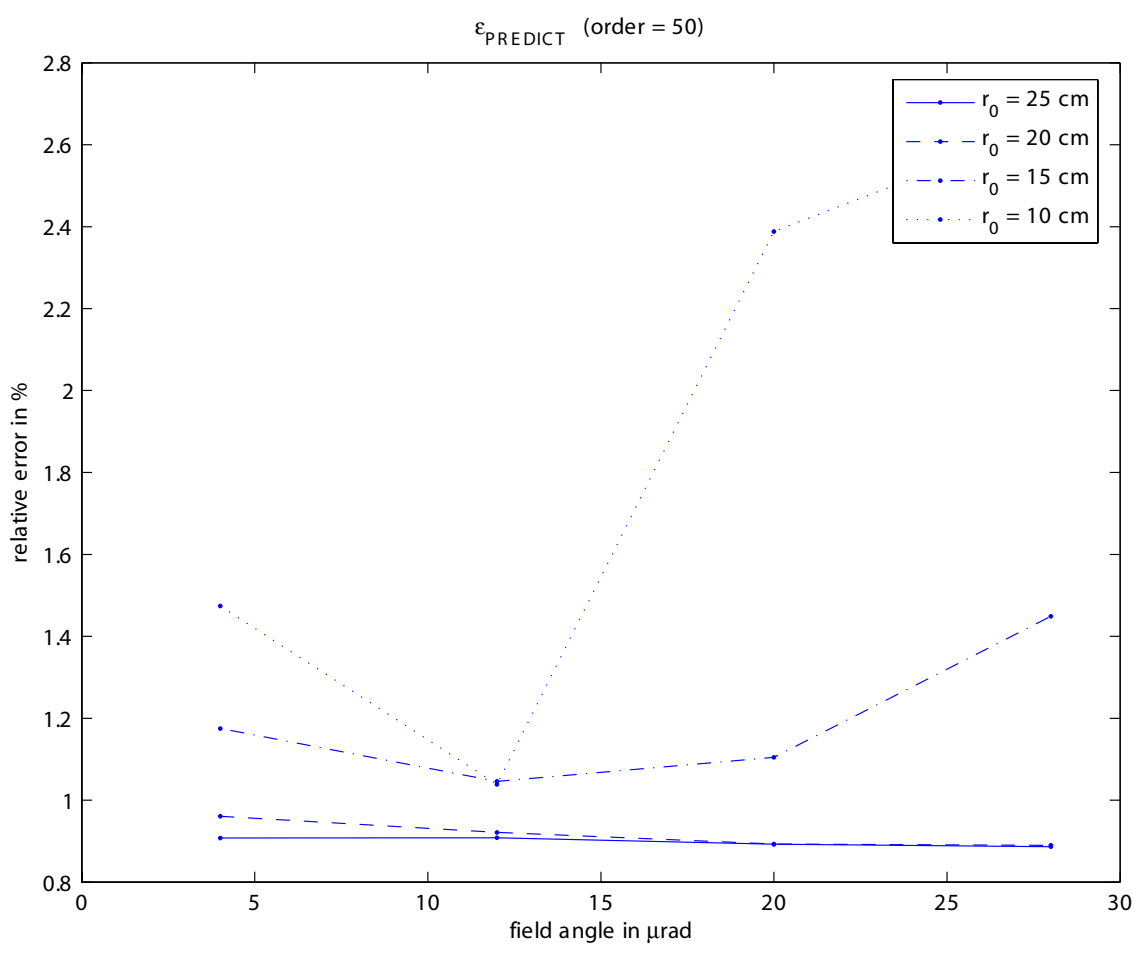

Figure 6.12: Prediction error $\epsilon_{P R E D I C T}$ vs. field angle.

for $\theta_{\text {predict }} \in \Theta_{\text {predict }}$. Figure 6.12 presents the prediction error as a function of the field angle, for each $r_{0}$. First, we can notice that the prediction error never exceeds $2.7 \%$, for any field angle or $r_{0}$. Although $\epsilon_{P R E D I C T}$ for $r_{0}=25$ and $20 \mathrm{~cm}$ slightly decreases with the field angle, the prediction error increases rapidly for $r_{0}=15 \mathrm{~cm}$ and to a larger extent for $r_{0}=10 \mathrm{~cm}$. This can be explained by the fact that for smaller $r_{0}$, the shape of the PSF (amplitude and width) changes at a faster rate (see section 6.3.3). Thus, the coefficients $a_{i}$ and $b_{i}$ are harder to predict for smaller $r_{0}$. To remedy this problem, a solution is to add information to the system by knowing more stars (and so PSF's) when $r_{0}$ is small. 


\subsection{Conclusion}

First, we introduced a model for the PSF based on the decomposition of its crosssections in a series of Chebyshev polynomials. The polynomials coefficients are the parameters of the PSF model.

Second, we presented a technique that allows us to predict the long-exposure AOcorrected PSF as a function of the field angle. The technique is based on the interpolation of the parameters of the model. The predicted PSF is compared to the simulated for different field angles and the relative MS error never exceeds $2.7 \%$. 


\section{CHAPTER 7}

\section{Reconstruction of anisoplanatic adaptive optics images}

\subsection{Introduction}

In this chapter, simulated anisoplanatic adaptive optics images are reconstructed using the knowledge of the predicted space-varying PSF as described in chapter 6. This chapter does not intend to give an extensive description of image restoration techniques, but it aims to validate our PSF prediction technique. Two widely used image restoration techniques are investigated: the Tikhonov regularization method and the expectation maximization algorithm. The deconvolution results using the space-varying predicted off-axis PSF are compared to deconvolution results using the space-invariant on-axis PSF.

This chapter is organized as follows. First, the approach used to deconvolve anisoplanatic images, based on block-processing, is presented. Second, the imaging model for each block, as well as the details of the block-processing technique are given. Two image restoration techniques are introduced and their reconstruction results are presented. The last section presents conclusions. 


\subsection{Approach}

The reconstruction method for anisoplanatic adaptive optics images consists of 4 steps:

- Step 1: Split the large field of view (FOV) image into small blocks in which turbulence-induced distortion is approximately constant. (Typically, the blocks are $\theta_{0} \times \theta_{0}$-sized),

- Step 2: Predict the local PSF (using the technique describe in chapter 6),

- Step 3: Deconvolve each block sequentially,

- Step 4: Reassemble the blocks to form the reconstructed image.

Steps 2 and 3 are repeated for each $\theta_{0}$-sized block of the image. Figure 7.1 gives a block diagram of the reconstruction method.

\subsection{Imaging model}

\subsubsection{Anisoplanatic conditions}

In the case of incoherent light, the model for the noise free intensity of an image $i\left(x_{i}, y_{i}\right)[14]$ is:

$$
i\left(x_{i}, y_{i}\right)=\iint h\left(x_{o}, y_{o} ; x_{i}, y_{i}\right) o\left(x_{o}, y_{o}\right) d x_{o} d y_{o},
$$

where $\left(x_{o}, y_{o}\right)$ and $\left(x_{i}, y_{i}\right)$ are the coordinates respectively in the object plane and the image plane. The object and image intensity distributions are respectively denoted $o\left(x_{o}, y_{o}\right)$ and $i\left(x_{i}, y_{i}\right)$, and $h\left(x_{o}, y_{o} ; x_{i}, y_{i}\right)$ is the space-varying PSF of the imaging system. 


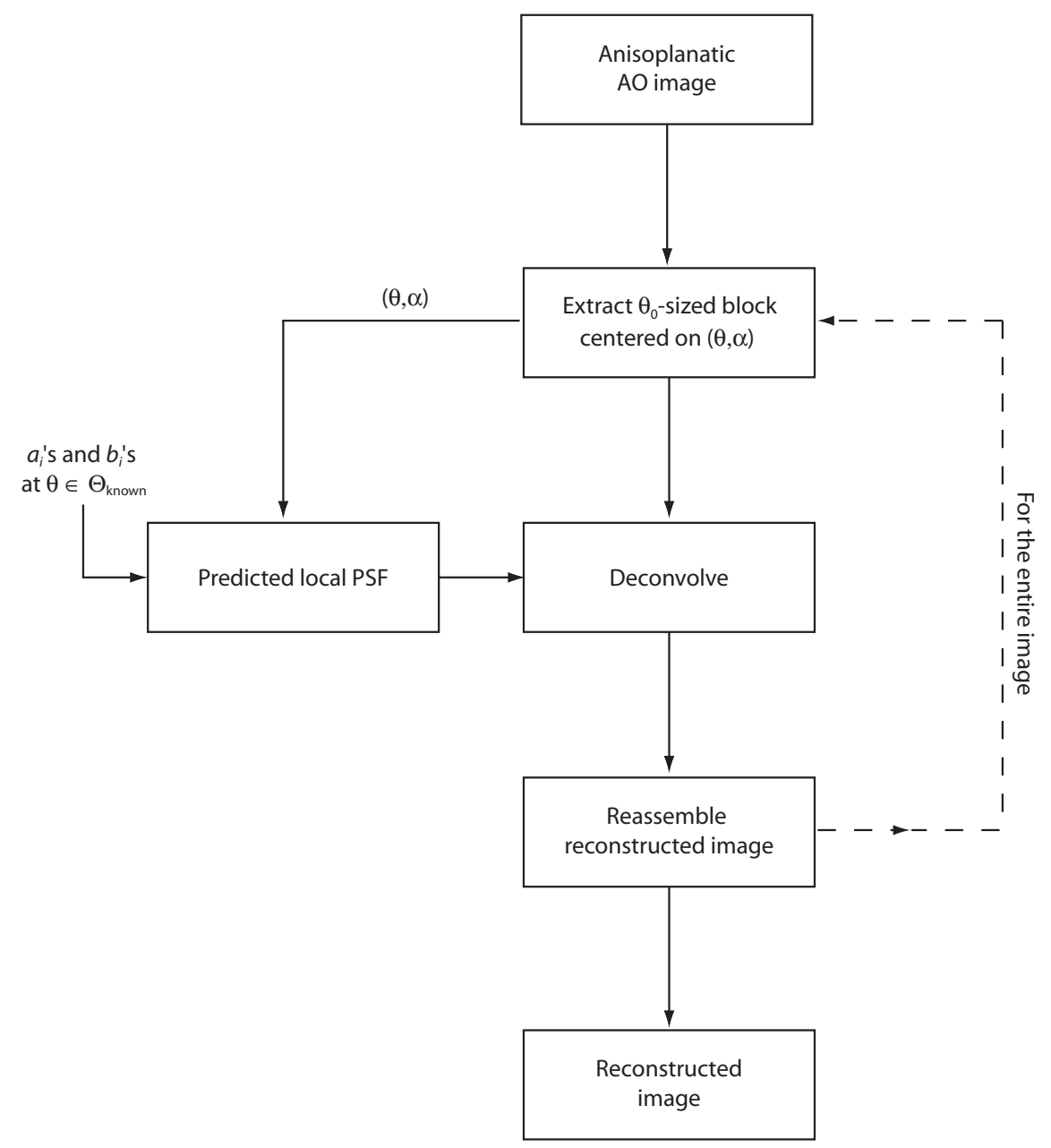

Figure 7.1: Block diagram of the block-processing method. 


\subsubsection{Isoplanatic conditions}

Under isoplanatic conditions, the turbulence-induced wavefront distortion is approximately constant over the entire image plane. The PSF is then space-invariant and can be denoted $h\left(x, y, x_{o}, y_{o}\right)=h\left(x_{o}-x ; y_{o}-y\right)$. The imaging model then becomes:

$$
i(x, y)=h(x, y) * o(x, y)
$$

where $*$ is the notation for the convolution operator. In the case of large FOV AOcorrected images, we are under anisoplanatic conditions. However, if we consider a small block of the image (typically of size $\theta_{0} \times \theta_{0}$ ), the turbulence-induced phase distortion is approximately constant and the imaging model given by Eq. 7.2 can be used.

\subsubsection{Noise}

Measured data are noisy. In addition to the photon noise (see section 2.4.4), we include additive noise to our model. A realistic imaging model [68] for a small block $\theta_{0}$-sized is

$$
i(x, y)=h(x, y) * o(x, y)+n(x, y)+n_{b}(x, y)
$$

where $n(x, y)$ denotes the light detector readout noise (see section 2.4.5), and $n_{b}(x, y)$ denotes the background noise. The readout noise is a Gaussian-distributed random variable accounting for the noise that is present in the readout electronic of light detectors $[16,17]$. The background noise is Poisson-distributed random variable accounting for the light arising from the background of the object of interest. The knowledge of $n(x, y)$ and $n_{b}(x, y)$ is limited to information of statistical nature: mean and variance. In this chapter, we simulate anisoplanatic images with no background noise. 


\subsubsection{Imaging model for a $\theta_{0}$-sized block}

The imaging model given by Eq. 7.3 can be written in discrete form:

$$
\mathbf{i}=\mathbf{H o}+\mathbf{n},
$$

where $\mathbf{i}, \mathbf{o}$, and $\mathbf{n}$ represent $N^{2}$-dimensional column vectors formed by staking the rows of the $N \times N$ discretized functions $i(x, y), o(x, y)$, and $n(x, y) . N$ is defined such that

$$
N \geq \frac{\theta_{0}}{\theta_{D A S}}
$$

where $\theta_{D A S}$ is the detector angular subtense. In our simulation, $N=64$ pixels. The matrix $\mathbf{H}$ is of dimension $N^{2} \times N^{2}$ and is called the impulse response matrix. It consists of $N^{2}$ partitions, each partition being of size $N \times N$ and ordered according to [69]

$$
\mathbf{H}=\left[\begin{array}{lllll}
\mathbf{H}_{0} & \mathbf{H}_{N-1} & \mathbf{H}_{N-2} & \cdots & \mathbf{H}_{1} \\
\mathbf{H}_{1} & \mathbf{H}_{0} & \mathbf{H}_{N-1} & \cdots & \mathbf{H}_{2} \\
\mathbf{H}_{2} & \mathbf{H}_{1} & \mathbf{H}_{0} & \cdots & \mathbf{H}_{3} \\
\vdots & \vdots & \vdots & \ddots & \vdots \\
\mathbf{H}_{N-1} & \mathbf{H}_{N-2} & \mathbf{H}_{N-3} & \cdots & \mathbf{H}_{0}
\end{array}\right]
$$

Each partition $\mathbf{H}_{j}$ is constructed from the $j$ th row of the PSF $h(x, y)$, as follows [69]

$$
\mathbf{H}_{j}=\left[\begin{array}{lllll}
h(j, 0) & h(j, N-1) & h(j, N-2) & \cdots & h(j, 1) \\
h(j, 1) & h(j, 0) & h(j, N-1) & \cdots & h(j, 2) \\
h(j, 2) & h(j, 1) & h(j, 0) & \cdots & h(j, 3) \\
\vdots & \vdots & \vdots & \ddots & \vdots \\
h(j, N-1) & h(j, N-2) & h(j, N-3) & \cdots & h(j, 0)
\end{array}\right] .
$$

Each partition $\mathbf{H}_{j}$ is a circulant matrix. The blocks $\mathbf{H}_{j}$ of $\mathbf{H}$ are subscripted in a circular manner. For this reason, the matrix $\mathbf{H}$ is called a block-circulant matrix. 


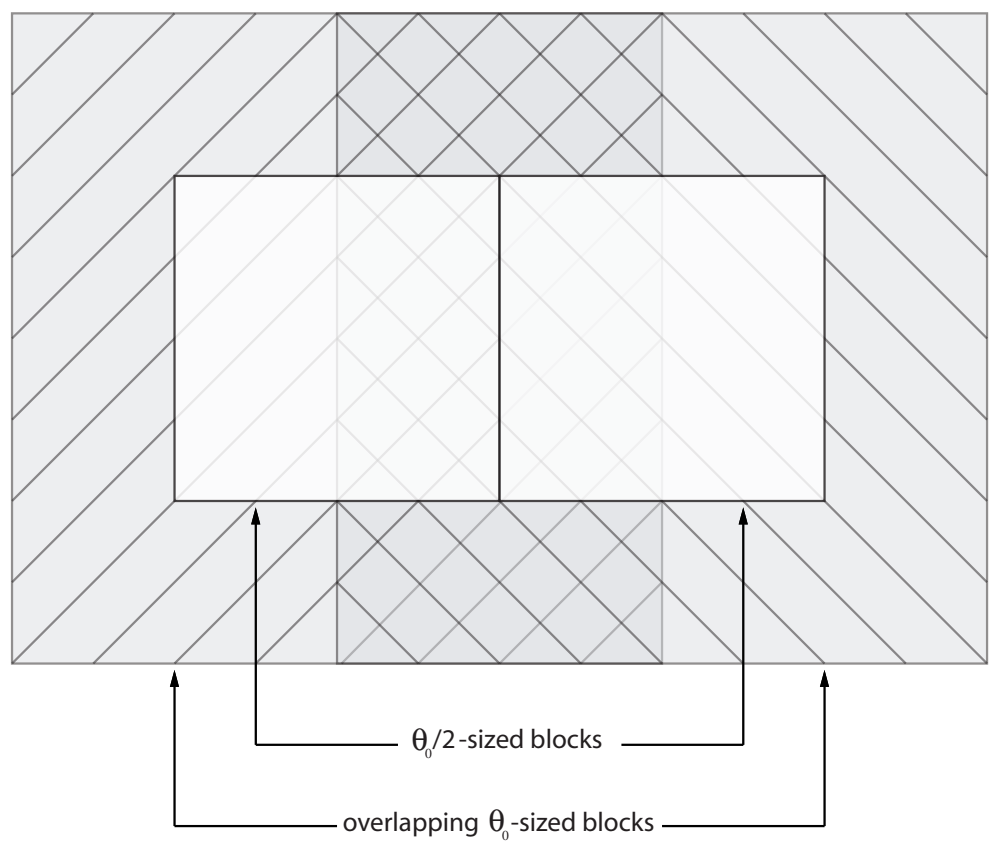

Figure 7.2: Block deconvolution of overlapping blocks.

\subsection{Block-processing}

The first step of the block processing method is to split the image in small blocks, of size approximately $\theta_{0} \times \theta_{0}$. The reason for splitting the image in small blocks is because in each block the PSF is approximately space-invariant and therefore, we can use the imaging model of section 7.3.4, which simplifies the deconvolution process.

In the solving of inverse problems, some undesired artifacts usually appear [70]. One of them is the boundary effect [71], which gives bad reconstruction results at the borders of the image. To avoid this problem, we consider an image block of size $N \times N$ pixels, reconstruct it, and then keep only the central $N / 2 \times N / 2$ pixels block, using a rectangular window. This way, the boundary effect does not interfere with deconvolution results. Thus, when block-deconvolving an image, we have to consider overlapping blocks. Figure 7.2 shows how overlapping blocks are extracted from the image to reconstruct. 


\subsection{Image reconstruction}

\subsubsection{Introduction}

We now consider the problem of reconstructing each $\theta_{0}$-sized image block (Step 1 of the image restoration method described in section 7.4) using the predicted local PSF as detailed in chapter 6 . The image is reconstructed by blocks using two widely used methods for image restoration.

\subsubsection{Inverse problem}

Our inverse problem was stated in section 7.3.4, where $\mathbf{o}$ is the unknown. Inverse problems are typically ill posed $[70,72,73,74,75]$, which means that small changes in the input can cause large changes in the output. As a consequence, the solution obtained is often unstable, due to noise amplification. To avoid this inconvenience during the inverse process, we need to regularize the problem. The Tikhonov regularization method (also known as Wiener filtering) is commonly used to solve ill-posed inverse problems. The approach used in Tikhonov regularization is to introduce some additional information by enforcing a smoothness criterion about the solution. The Tikhonov regularization is studied into details in section 7.5.3. Another commonly used method to solve inverse problems is the expectation maximization (EM) algorithm $[76,77]$. The EM algorithm is a statistical method and is presented into details in section 7.5.4.

\subsubsection{Method 1: Tikhonov regularization}

The Tikhonov regularization minimizes the quantity $\|\mathbf{i}-\mathbf{H} \tilde{\mathbf{o}}\|^{2}$, in the presence of a smoothness constraint on the estimate of the object $\mathbf{o}$, denoted $\tilde{\mathbf{o}}$. This constraint is 
controlled through the parameter $\alpha$. The goal of the Tikhonov regularization is to minimize the quantity

$$
J(\tilde{\mathbf{o}})=\|\mathbf{i}-\mathbf{H} \tilde{\mathbf{o}}\|^{2}+\alpha\|\mathbf{M} \tilde{\mathbf{o}}\|^{2},
$$

where $\gamma=1 / \alpha$ is called the Lagrange multiplier, which controls the smothness of $\tilde{\mathbf{o}}$. Different choices of the matrix $M$ yield different solutions for $\tilde{\mathbf{o}}$ [69]. The matrix $M$ can chosen as the matrix taking the gradient or the Laplacian of the solution [78]. In the Tikhonov approach, $M$ is chosen as the identity matrix. The parameter $\alpha$ is to be chosen. For a small $\alpha$, the reconstructed image $\tilde{\mathbf{o}}$ is sharper. However, for the limit case $\alpha=0$, we have a direct inverse problem, in which the noise amplification problem arises. For large values of $\alpha$, the reconstructed image is less noisy but smoother and less accurate. A tradeoff has to be found between an accurate and a noisy result.

Minimizing Eq. 7.8 is equivalent to solve the following linear system

$$
\left(\mathbf{H}^{T} \mathbf{H}+\alpha \mathbf{M}^{T} \mathbf{M}\right) \tilde{\mathbf{o}}=\mathbf{H}^{T} \mathbf{i} .
$$

Thus, using the Tikhonov regularization $(\mathbf{M}=\mathbf{I})$, the estimate of $\mathbf{o}$ for a fixed $\alpha$ is given by

$$
\tilde{\mathbf{o}}=\left(\mathbf{H}^{T} \mathbf{H}+\alpha \mathbf{I}\right)^{-1} \mathbf{H}^{T} \mathbf{i}
$$

\subsubsection{Method 2: Expectation maximization (EM) algorithm}

The EM technique has been widely used in the literature $[76,77,79,80,81,82,83]$. In this method, the PSF is assumed to be perfectly known. The PSF is normalized such that

$$
\sum_{i=1}^{N^{2}} h_{i}=1,
$$

where $h_{i}$ is the $i^{\text {th }}$ element of the $N^{2}$-dimensional column vector $\mathbf{h}$ formed by staking the rows of the $N \times N$ PSF $h(x, y)$. Thus, the normalized PSF can be regarded as a probability density function (PDF). In the case of a Poisson noise distribution, the 
EM algorithm can be shown as a maximum-likelihood (ML) solution. However, in the case of non-Poisson noise, the EM algorithm does not give a ML solution, but is still useful for image reconstruction. If $\mathbf{o}$ and $\mathbf{i}$ denote respectively the object and the image intensity, and $\mathbf{H}$ the impulse response matrix, the EM algorithm is given by the recursive formula

$$
\tilde{\mathbf{o}}_{n}^{k}=\tilde{\mathbf{o}}_{n}^{k-1}\left(\frac{1}{\sum_{m} \mathbf{H}_{m n}}\right) \sum_{m}\left(\mathbf{H}_{m n} \cdot \frac{\mathbf{i}_{m}}{\sum_{l} \mathbf{H}_{m l} \tilde{\mathbf{o}}_{l}^{k-1}}\right),
$$

for $i \in\left[1 ; N^{2}\right]$, and where $\mathbf{H}_{i j}$ is the $i^{\text {th }}$ element of $j^{t h}$ column of the matrix $\mathbf{H}$. The element $(i, j)$ of the matrix $\mathbf{H}$ can be interpreted as the probability that a photon arising from the $i$ th pixel location in the object plane falls onto the ${ }^{t h} j$ pixel location in the image plane.

For the first iterations of the algorithm, only the low spatial frequencies of the object are reconstructed. The higher spatial frequencies are reconstructed for a larger number of iterations. If we compute too many iterations of the algorithm, the restored image can sometimes have a speckled appearance. These speckles do not represent any real structure in the image, but are artifacts due to the noise amplification problem. Therefore, we have to find a tradeoff between the accuracy of the reconstructed object and the noise amplification problem [84].

\subsection{Results}

\subsubsection{Introduction}

The two restoration methods were applied to a simulated anisoplanatic image. The image considered is a simulated stellar field formed by computing the image of point sources of different amplitudes (between 0.5 and 1) randomly distributed in the object plane. Figure 7.3 shows the simulated star field image with no photon noise or additive 

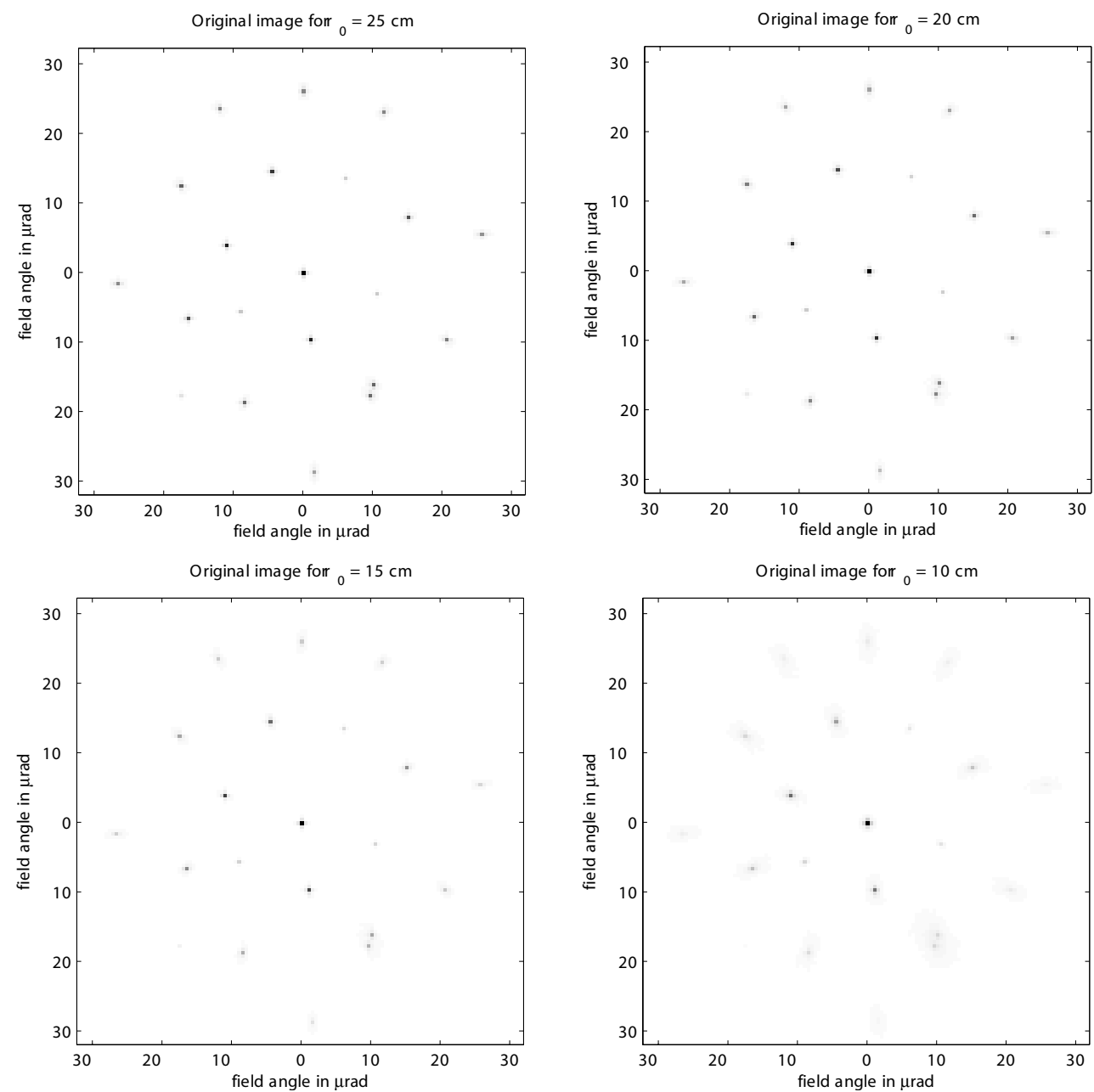

Figure 7.3: Simulated images for different $r_{0}$ 's.

noise for different $r_{0}$. In the figure, we can clearly notice the effect of anisoplanatism in the case $r_{0}=10$ and $15 \mathrm{~cm}$ : the stars with the largest angular separation from the reference beacon become very hard to distinguish.

The images are deconvolved using the predicted local PSF, and, for comparison, with the space invariant PSF (on-axis PSF). The results using both variant and invariant PSF's are compared to the original object using a MS error metric. Finally, the influence of the signal-to-noise ratio (SNR) on the results is studied. 


\subsubsection{Error metric}

The quality of the reconstructed image is measured by computing the relative MS error between the reconstructed object and the object. The error metric is defined by

$$
\epsilon_{\text {RECONS }}^{2}=\frac{\sum_{x} \sum_{y}\|\tilde{o}(x, y)-o(x, y)\|^{2}}{\sum_{x} \sum_{y}\|o(x, y)\|^{2}} .
$$

This quantity gives a measurement of the quality of the results averaged over the entire reconstructed image.

\subsubsection{Results for the Tikhonov regularization}

Figure 2.1 and Figure 2.2 (Appendix B) give the reconstruction error $\epsilon_{R E C O N S}$ as a function of the Tikhonov regularization parameter $\alpha$ for different $r_{0}$. From those figures, we can make different remarks.

- The first thing to notice is that the reconstruction error using the predicted local PSF is overall smaller than the reconstruction error using the space-invariant on-axis PSF, whatever the value of the Fried parameter $r_{0}$.

- The best results in term of MS error are obtained for larger $r_{0}$. For large $r_{0}$, the isoplanatic angle is larger and the PSF varies more slowly as the field angle increases. This makes the prediction of the PSF by interpolation more accurate and therefore gives better reconstruction results.

- We define $\alpha_{\min }$ such that

$$
\epsilon_{R E C O N S}^{2}\left(\alpha_{m i n}\right)=\min \left\{\epsilon_{R E C O N S}^{2}\right\}
$$

For $\alpha_{\min }=0$, the deconvolution is equivalent to direct inversion. From Figure 2.1 and Figure 2.2, we can see that for large $r_{0}$, we have $\alpha_{\min }=0$. For smaller $r_{0}$, we have $\alpha_{\min }>0$. We saw in section 6.3.3 that the amplitude of the PSF decreases. 
As a consequence, the additive noise component of the signal is relatively larger amplitude compared to the data component of the image (see definition of the SNR in Eq. 2.62). Therefore, the inverse problem is not as well-posed as for large $r_{0}$ and the problem needs to be regularized. For this reason, we have $\alpha_{\min }>0$.

Figure 7.4 gives the reconstruction result for $\mathrm{SNR}=10000$ and $r_{0}=15 \mathrm{~cm}$. In Appendix $\mathrm{B}$, reconstructed images for different SNR and different $r_{0}$ are given by Figures 2.7 to 2.18. In each case, the images are deconvolved for a smoothness coefficent $\alpha=\alpha_{\min }$.

\subsubsection{Results for the EM algorithm}

Figure 7.5 gives the reconstruction result for $\mathrm{SNR}=10000$ and $r_{0}=15 \mathrm{~cm}$. In Appendix B, reconstructed images for different SNR and different $r_{0}$ are given Figures 2.19 to 2.30. In each case, the algorithm is iterated until $\epsilon_{R E C O N S}^{2}$ reaches its minimum.

Figure 2.3 and Figure 2.4 (Appendix B) give the reconstruction error $\epsilon_{R E C O N S}$ as a function of the number of iterations of the EM algorithm for different $r_{0}$. Several aspects are to be noticed.

- The first thing to notice is that, like for the Tikhonov regularization, the reconstruction error is smaller when using the space-varying predicted PSF than the on-axis PSF.

- As the seing conditions degrade (small $r_{0}$ ), the reconstruction error increases, for the same reasons than explained in section 7.6.3.

- The reconstruction error usually reaches its minimum after few iterations. For example, for $r_{0}=25 \mathrm{~cm}, \epsilon_{R E C O N S}^{2}$ is minimized for 12 to 18 iterations. However, for smaller $r_{0}, \epsilon_{R E C O N S}^{2}$ takes more iterations to reach its minimum. 

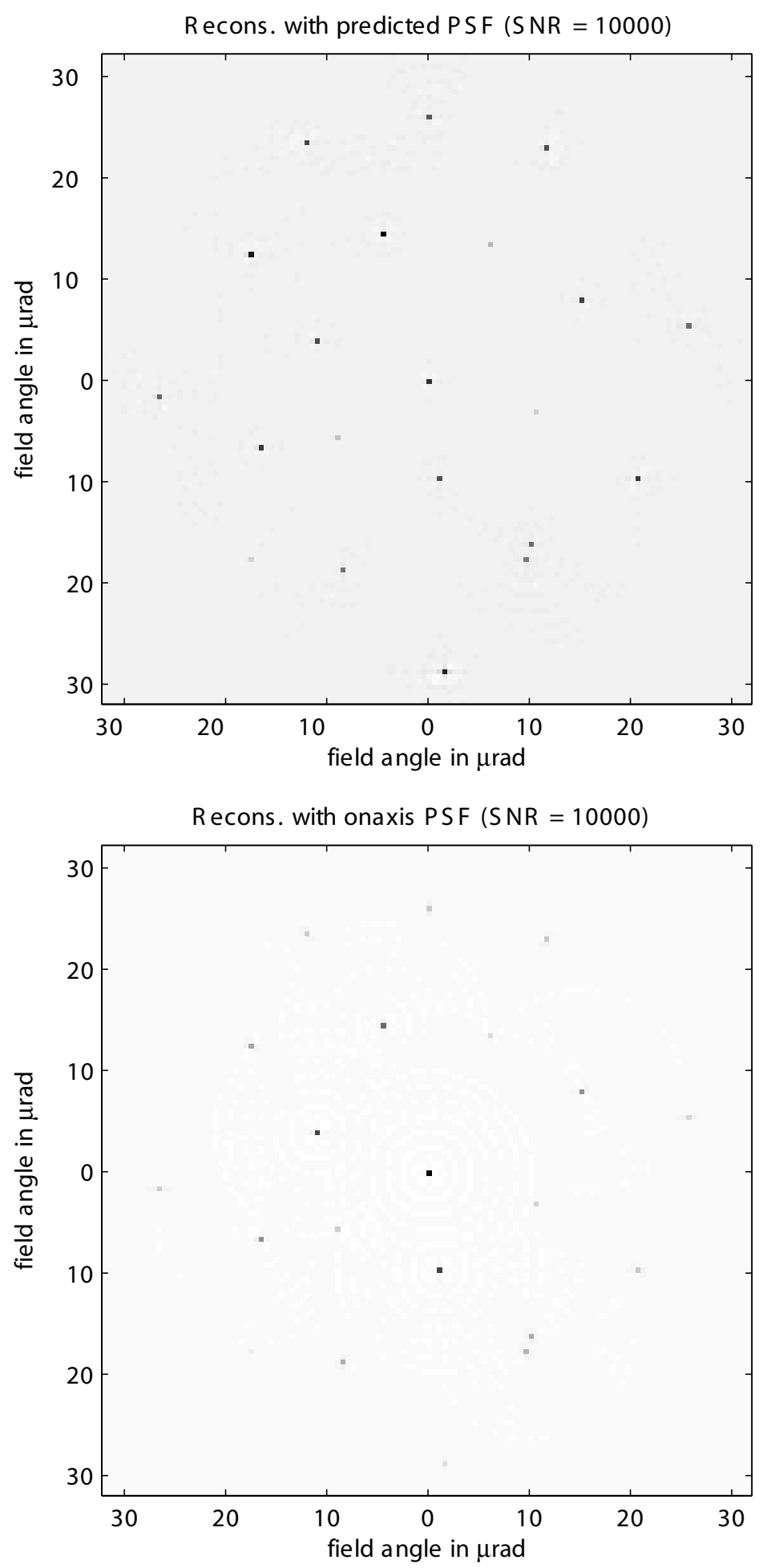

Figure 7.4: Reconstructed images using the Tikhonov regularization with the predicted $P S F$ and the on-axis PSF for $S N R=10000$ and $r_{0}=15 \mathrm{~cm}$. 

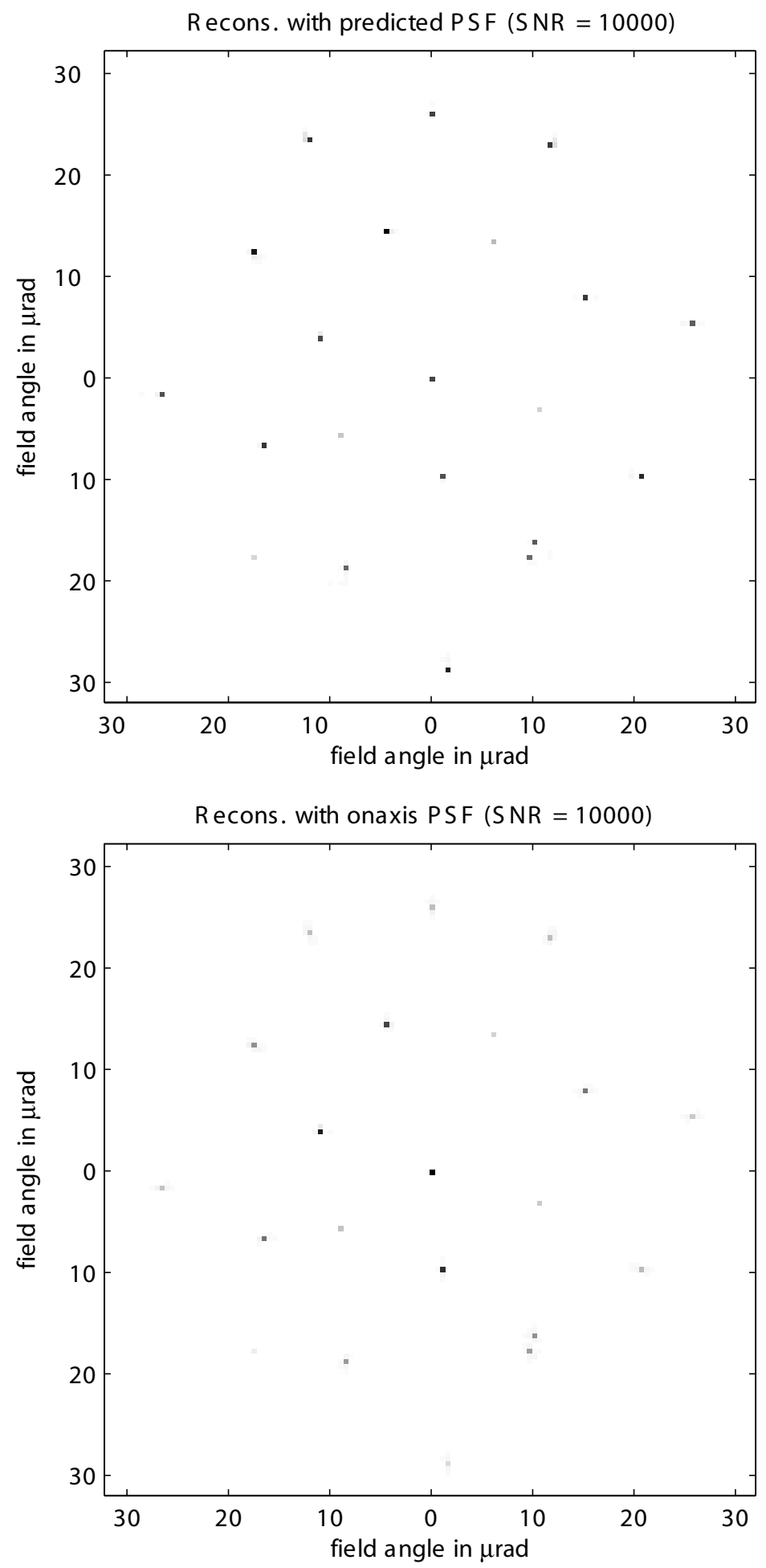

Figure 7.5: Reconstructed images using the EM algorithm with the predicted PSF and the on-axis PSF for $S N R=10000$ and $r_{0}=15 \mathrm{~cm}$. 


\subsubsection{Influence of the SNR}

The SNR gives a measure of the relative strength of the image and the random component of an image. It is a way to quantify the effect of noise on an image. Figure 2.5 and Figure 2.6 (Appendix B) give respectively the reconstruction error as a function of the SNR in the case of the Tikhonov regularization method and the EM algorithm method. Whatever the reconstruction method, the quality of the reconstruction increases as the SNR increases. However, for a SNR greater than 100 the results do not improve. The reconstruction error does not decreases at the same rate for both methods: at low SNR, $\epsilon_{R E C O N S}$ decreases faster when using the EM algorithm. For example, for a SNR of 10, the EM algorithm gives a reconstruction error of $39 \%$ in the worst case, as opposed to $83 \%$ when using the Tikhonov regularization. For high SNR, the Tikhonov regularization gives better results though, as low as $\epsilon_{R E C O N S}=4 \%$.

\subsubsection{Comparison space-invariant/space-varying PSF decon- volution}

When reconstructing isoplanatic images, the PSF used is space-invariant. Typically, the PSF consider will be the on-axis PSF. We now want to compare the deconvolution results when using the on-axis PSF with the deconvolution results when using the spacevarying predicted PSF. We define the improvement factor $\xi$ which gives a measurement of the gain from the first technique to the second one, in term of the MS reconstruction error. The improvement factor $\xi$ is defined by

$$
\xi=\frac{\epsilon_{o n-a x i s}^{2}-\epsilon_{o f f-a x i s}^{2}}{\epsilon_{o n-a x i s}^{2}}
$$

where $\left.\epsilon_{R E C O N S}^{2}\right|_{O n-a x i s P S F}$ and $\left.\epsilon_{R E C O N S}^{2}\right|_{\text {PredictedPSF }}$ denote the reconstruction error $\epsilon_{R E C O N S}$ when deconvolving respectively with the on-axis and the predicted off-axis PSF. 
Tables 7.1 to 7.3 give the improvement factor $\xi$ for different SNR. Several remarks are to be made.

- We can first notice that in all tables, the improvement factor $\xi$ is positive, which means that the reconstruction results were effectively improved, in term of MS error.

- The best improvement factor is obtained with the Tikhonov regularization for high SNR $\left(\mathrm{SNR}=10^{4}\right)$. In this case, the improvement factor is greater than $80 \%$. However, for lower SNR, the EM algorithm gives better results.

\subsection{Conclusion}

We presented a block-processing method for deconvolution of large FOV AO-corrected images, based on the knowledge of the PSF as a function of the field angle. Two reconstruction techniques had been studied: the Tikhonov regularization and the EM algorithm. The performance of both reconstruction methods had been estimated in term of MS error between the reconstructed image and the object. The reconstruction results were presented and the both techniques were showed an improvement of the original image.

Furthermore, in both cases, the predicted space-varying PSF gave better reconstruction error than the on-axis PSF (as much as 84.8\%), which validates the method for prediction of the PSF as a function of the field angle, presented in chapter 6 .

The influence of the Fried parameter as well as the SNR on results had been studied in both reconstruction techniques.

In both cases, the performance decreases as the Fried parameter gets smaller. However, we obtained better results with the EM algorithm for bad seeing conditions (small 
$\left.r_{0}\right)$.

In both cases, the performance decreases as the SNR gets smaller. The Tikhonov regularization gave the best reconstruction results for high SNR. However, when the SNR is low (smaller than 10), the EM algorithm gave much smaller reconstruction errors. 


\begin{tabular}{|c||c|c|}
\hline \multicolumn{1}{|c||}{} & \multicolumn{2}{c|}{ Method } \\
$r_{0}(\mathrm{~cm})$ & Tikhonov Regularization & EM algorithm \\
\hline \hline 25 & $\mathbf{8 2 . 9} \%$ & $71.4 \%$ \\
20 & $\mathbf{8 4 . 8} \%$ & $78.3 \%$ \\
15 & $\mathbf{8 3 . 1} \%$ & $80.5 \%$ \\
10 & $34.6 \%$ & $\mathbf{7 3 . 8} \%$ \\
\hline
\end{tabular}

Table 7.1: Improvement factor $\xi$ for $S N R=10^{4}$.

\begin{tabular}{|c||c|c|}
\hline \multicolumn{1}{|c||}{} & \multicolumn{2}{c|}{ Method } \\
$r_{0}(\mathrm{~cm})$ & Tikhonov Regularization & EM algorithm \\
\hline \hline 25 & $45.9 \%$ & $\mathbf{6 7 . 0} \%$ \\
20 & $49.2 \%$ & $\mathbf{7 4 . 3} \%$ \\
15 & $46.0 \%$ & $\mathbf{7 3 . 0} \%$ \\
10 & $17.3 \%$ & $\mathbf{6 6 . 0} \%$ \\
\hline
\end{tabular}

Table 7.2: Improvement factor $\xi$ for $S N R=1$.

\begin{tabular}{|c||c|c|}
\hline \multicolumn{1}{|c||}{} & \multicolumn{2}{c|}{ Method } \\
$r_{0}(\mathrm{~cm})$ & Tikhonov Regularization & EM algorithm \\
\hline \hline 25 & $25.1 \%$ & $\mathbf{6 5 . 4} \%$ \\
20 & $31.8 \%$ & $\mathbf{7 0 . 1} \%$ \\
15 & $28.9 \%$ & $\mathbf{7 2 . 0} \%$ \\
10 & $7.2 \%$ & $\mathbf{6 4 . 2} \%$ \\
\hline
\end{tabular}

Table 7.3: Improvement factor $\xi$ for $S N R=0.5$. 


\section{CHAPTER 8}

\section{Experiment}

\subsection{Introduction}

In this chapter, we describe the results of an experiment designed to validate the results obtained through simulations in chapter 7 . The experiment was designed to measure anisoplanatic adaptive optics images, using the US Air Force Advanced Electro-Optical System (AEOS), atop Mt. Haleakala on the island of Maui, HI. The images obtained were reconstructed using the technique introduced in chapter 7 .

In section 8.2, the experimental setup is described as well as the data obtained. In section 8.3 , the data pre-processing method is given. In section 8.4 , the data are reconstructed using the image restoration technique presented in the previous chapter, and the results are presented in section 8.5. Section 8.6 is the conclusion.

\subsection{Data}

The data were measured at the Maui Space Surveillance System (MSSS) using the 3.67-meter AEOS telescope [85]. AO-corrected images of binary stars with different angular separations and magnitudes had been measured. Two series of measurements had been organized. The first one occurred from October $24^{\text {th }}$ to October $28^{\text {th }}, 2004$. 
The second one occurred from February $14^{\text {th }}$ to February $17^{\text {th }}$, 2005. A total of 28 binary stars were observed. For each binary star, we denote star A the brightest star, and star B the dimmest one. Star A is always used as the reference beacon for the AO system. Table 8.1 gives their name, angular separation, magnitudes $\left(m_{A}\right.$ and $\left.m_{B}\right)$, exposure time and Fried parameter during measurements. The field of view for all images is $51.2 \times 51.2 \mu$ radians. We denote $N_{f}$ the number of frames taken for a binary star. For each binary star, we have $N_{f}=100$ frames. Figure 8.1 gives examples of raw data.

\subsection{Data pre-processing}

For each binary star, we have $N_{f}$ frames. We pre-process these frames in order to obtain a single image for each binary star. The first step is to shift spatially in order to have the peak value of the brightest star at the center of the image plane. This step

will simplify later on the deconvolution process. Each pre-processed frame $i_{p r e}^{k}(x, y)$ is given by

$$
i_{p r e}^{k}(x, y)=i^{k}\left(x-x_{\max }, y-y_{\max }\right),
$$

where $i^{k}(x, y)$ denotes the $k^{\text {th }}$ measured frame, and $\left(x_{\max }, y_{\max }\right)$ denotes the spatial coordinates of the peak value in the image. The second step of the pre-processing is to average all the frames and to subtract the background noise. For each binary star, the pre-processed image $i_{\text {pre }}(x, y)$ is given by

$$
i_{\text {pre }}(x, y)=\frac{1}{N_{f}}\left(\sum_{k=1}^{N_{f}} i_{\text {pre }}^{k}(x, y)\right)-n_{\text {back }}(x, y),
$$

where $N_{f}$ denotes the number of frames, and $n_{\text {back }}(x, y)$ denotes the background noise. To obtain the background noise, we measure a dark section of the sky and compute the average over the entire image, and over each frame. Figure 8.2 gives examples of pre-processed images of the binary stars. 

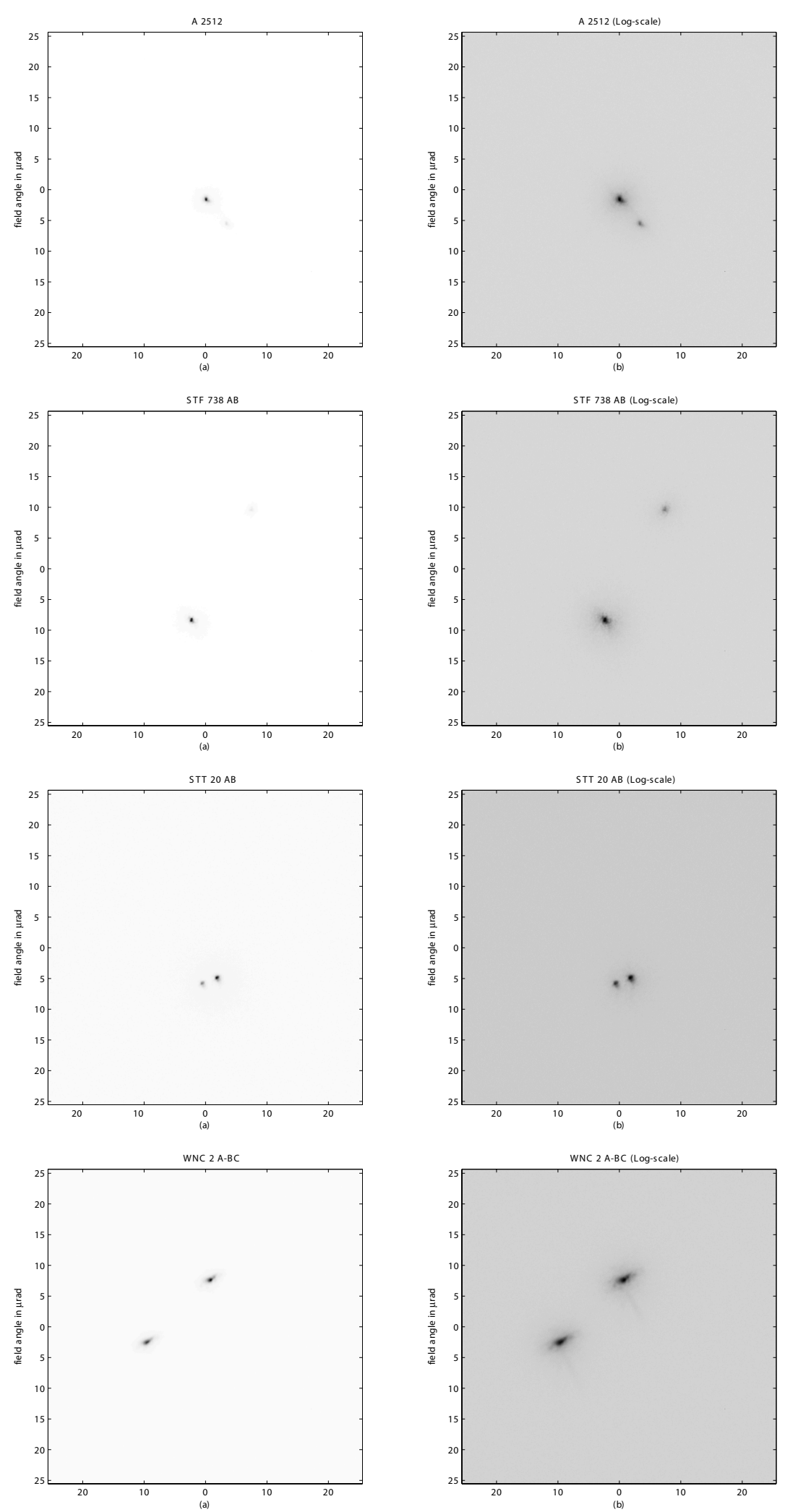

Figure 8.1: Examples of raw data. 


\begin{tabular}{|c||c|c|c|c|c|}
\hline Star name & Sep. $(\mu \mathrm{rad})$ & $m_{A}$ & $m_{B}$ & Exp. $(\mathrm{ms})$ & $r_{0}(\mathrm{~cm})$ \\
\hline A 2512 & 5.1 & 7.3 & 9.5 & 1000 & 8.1 \\
A 3010 & 4.8 & 5.8 & 5.8 & 250 & 6.0 \\
BU 311 & 2.2 & 6.7 & 7.1 & 1500 & 8.0 \\
BU 396 & 7.3 & 6.1 & 8.6 & 100 & 31.0 \\
BU 535 & 4.8 & 3.9 & 6.7 & 120 & 22.4 \\
BU 1052 & 2.7 & 6.7 & 8.2 & 2000 & 8.5 \\
DA 3 & 4.2 & 7.3 & 8.5 & 100 & 10 \\
DA 4 & 5.9 & 4.6 & 7.5 & 100 & 5.5 \\
DA 5 Aa-B & 8.3 & 3.6 & 4.9 & 100 & 8.7 \\
HDS 509Aa & 3.5 & 5.8 & 7.9 & 150 & 9.6 \\
HJ 3375 & 21.8 & 6.6 & 8.5 & 2000 & 19.7 \\
HJ 3589 & 24.2 & 6.6 & 9.3 & 250 & 19.6 \\
HJ 3752 AB & 16.3 & 5.4 & 6.6 & 2000 & 4.1 \\
STF 268 & 14.5 & 6.7 & 8.5 & 1000 & 23.0 \\
STF 311 AB & 17.0 & 5.3 & 7.9 & 250 & 15.4 \\
STF 535 & 5.3 & 6.9 & 8.3 & 1000 & 22.4 \\
STF 636 & 17.4 & 7.1 & 8.5 & 100 & 3.5 \\
STF 661 & 10.1 & 4.4 & 6.8 & 100 & 7.9 \\
STF 708 & 13.0 & 7.7 & 8.9 & 3000 & 12.1 \\
STF 712 AB & 15.1 & 6.7 & 8.6 & 600 & 10.3 \\
STF 716 AB & 22.4 & 5.8 & 6.7 & 3000 & 4.7 \\
STF 734 AB & 7.5 & 6.7 & 8.2 & 300 & 9.9 \\
STF 738 AB & 20.5 & 3.5 & 5.5 & 300 & 9.5 \\
STF 742 & 19.4 & 7.1 & 7.5 & 5000 & 18.9 \\
STF 736 & 12.1 & 7.5 & 8.6 & 1000 & 21.0 \\
STT 09 AB & 9.7 & 6.9 & 9.7 & 175 & 21.3 \\
STT 515 AB & 2.4 & 4.6 & 5.6 & 250 & 23.8 \\
WNC 2A-BC & 14.5 & 6.9 & 10.0 & 300 & 9.9 \\
\hline
\end{tabular}

Table 8.1: Binary stars measured at the AEOS. 

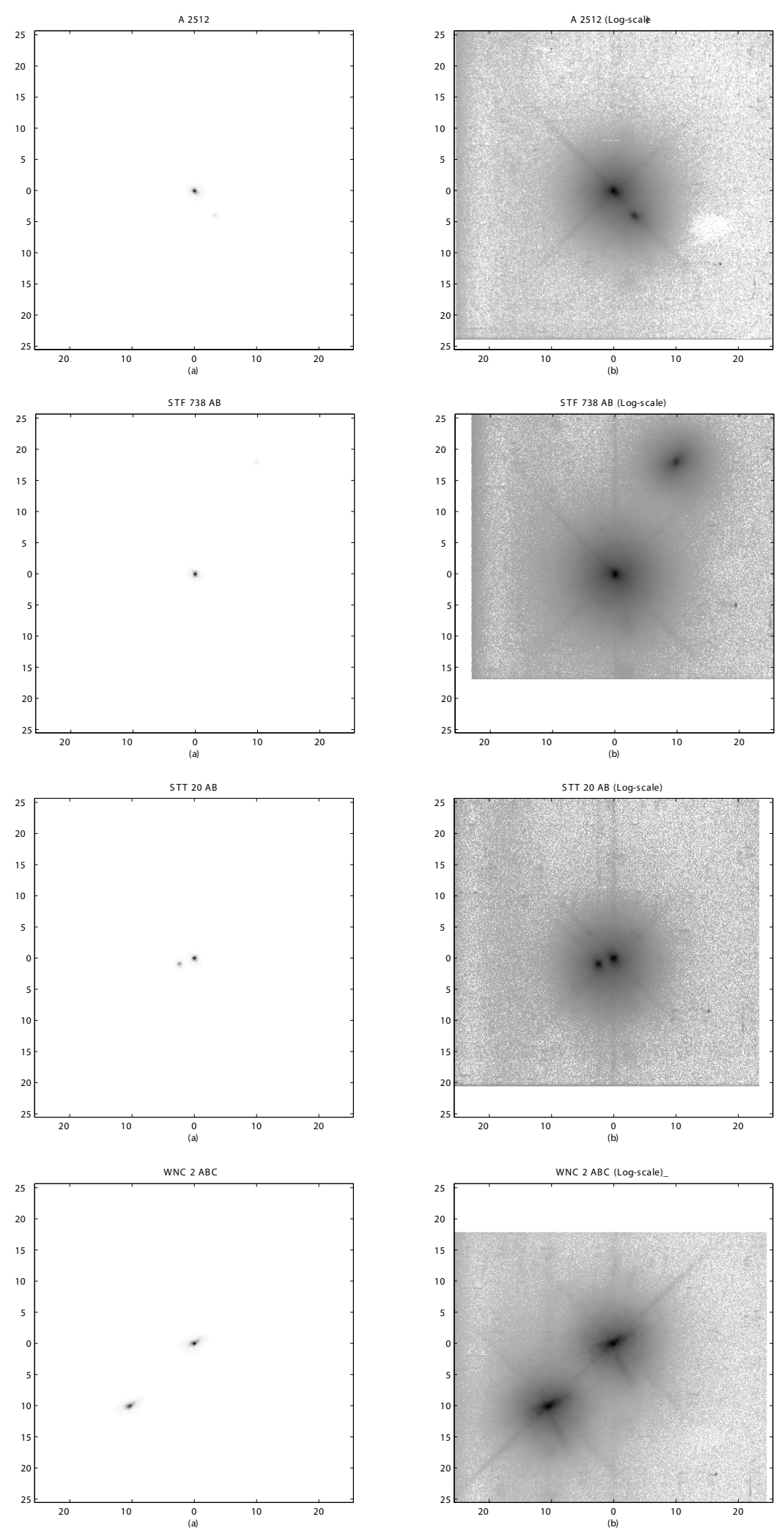

Figure 8.2: Pre-processed images of binary stars. 


\subsection{Observation of anisoplanatic effects on images}

\subsubsection{Width of the PSF}

We now want to observe the effects of anisoplanatism on AO-corrected images, as discussed in chapter 6. Each star (the object and not the image of the star) has an angular size much smaller than the angular resolution of the imaging system, therefore each star can be considered as a point source. Thus, the image of a star can be considered as a non-normalized PSF.

In section 6.3.2, we saw that anisoplanatism affects AO-corrected images by making the PSF broader, as the field angle with the reference beacon increases. Figure 8.3 shows the measured cross-sections of the on-axis PSF (reference beacon) and the off-axis PSF for four different stars. The PSF's are normalized such that the peak value equals to one. For each binary star, the angular separation $\theta$ between the two stars and the Fried parameter $r_{0}$ are different: (a) STF $738 \mathrm{AB}: \theta=20.5 \mu \mathrm{rad}\left(r_{0}=9.5 \mathrm{~cm}\right)$, (b) HJ 3752 AB: $\theta=16.3 \mu \mathrm{rad}\left(r_{0}=4.1 \mathrm{~cm}\right),(\mathrm{c})$ A 2512: $\theta=5.1 \mu \mathrm{rad}\left(r_{0}=8.1 \mathrm{~cm}\right)$, and $(\mathrm{d}) \mathrm{BU}$ 311: $\theta=2.2 \mu \mathrm{rad}\left(r_{0}=8 \mathrm{~cm}\right)$.

We can notice that for each binary star, as expected, the off-axis PSF is broader than the on-axis PSF. The larger the angular separation $\theta$ is, the broader the PSF becomes. Also, the Fried parameter is different for each binary star image (See Table 8.1). As $r_{0}$ gets smaller, the PSF becomes broader.

\subsubsection{Encircled energy}

The difference of magnitude between the two binary stars is related to their intensity by

$$
I_{B}=2.512^{\left(m_{A}-m_{B}\right)} I_{A}
$$



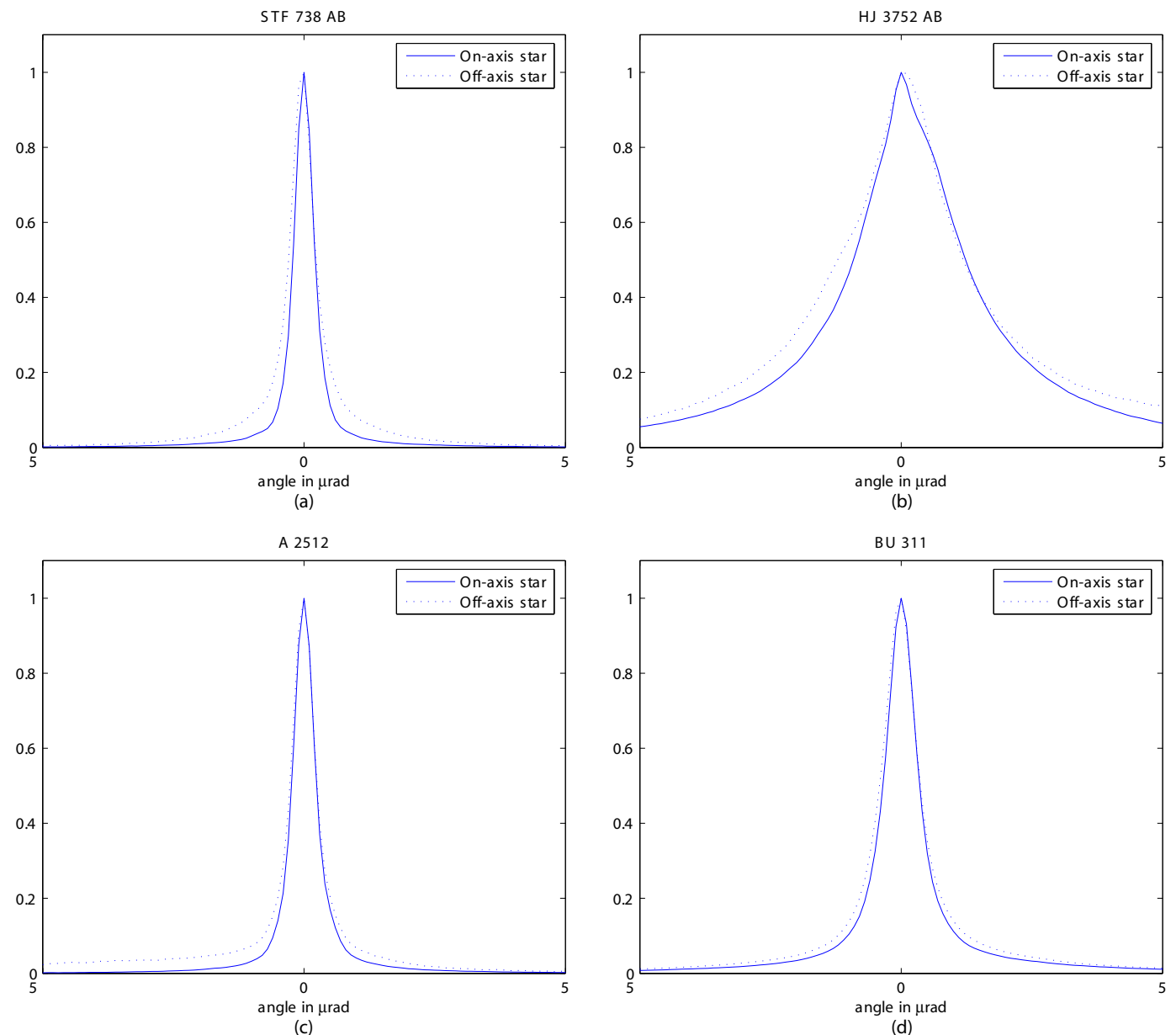

Figure 8.3: Cross-sections of different binary stars. 
where $m_{A}$ and $m_{B}$ denote the magnitudes of the stars, and $I_{A}$ and $I_{B}$ denote their intensities, or apparent brightness. Table 8.2 gives the normalized intensity for each star computed from Eq. 8.3, as well as the intensity measured from the data. Each intensity is normalized with respect to the intensity $I_{A}$. The intensity of each star is measured by computing the encircled energy in a disk of a given diameter, centered on the star. The diameter of the circle varies between $2 \mu \mathrm{rad}$ and $10 \mu \mathrm{rad}$ depending on the extent of the image of each star. In the case of the binary star HJ $3752 \mathrm{AB}$, the Fried parameter is small $\left(r_{0}=4.1 \mathrm{~cm}\right)$. As a consequence, the PSF is very broad (See Figure 8.3 (b)) and therefore the intensity distributions of both stars are overlaping. Thus the encircled energy for each star can not be measured.

\begin{tabular}{|c||c|c||c|c|}
\hline \multicolumn{1}{|c||}{ Star } & \multicolumn{2}{c||}{ Computed intensity } & \multicolumn{3}{c|}{ Measured intensity } \\
& $I_{A}$ & $I_{B}$ & $I_{\text {A meas. }}$ & $I_{\text {B meas. }}$ \\
\hline STF 738 AB & 1 & 0.1675 & 1 & 0.1643 \\
HJ 3752 AB & 1 & 0.3499 & 1 & PSF too broad to measure \\
A 2512 & 1 & 0.1355 & 1 & 0.1353 \\
BU 311 & 1 & 0.6792 & 1 & 0.7425 \\
\hline
\end{tabular}

Table 8.2: Computed and measured intensities for four binary stars.

We can notice that the measured intensity and the computed intensity are very similar. Anisoplanatism has the following effect on the AO-corrected PSF: it broadens the PSF and decreases its peak value as the field angle increases. However, it does not change the total energy coming from each star.

\subsubsection{Peak value of the PSF}

For different binary stars, we look at the peak value of the PSF at various field angles. We assume the peak values of two stars $\mathrm{A}$ and $\mathrm{B}$, denoted $K_{A}$ and $K_{B}$, to be related 
by Eq. 8.3. Table 8.3 gives the normalized peak values of the PSF measured from the data, and computed from Eq. 8.3. Each peak value is normalized by the peak value of the on-axis PSF.

\begin{tabular}{|c||c|c|c|c|c|}
\hline \multirow{2}{*}{ Star } & \multirow{2}{*}{$K_{A}$} & \multicolumn{2}{|c|}{$K_{B}$} & Difference & Angular sep. \\
& & th. & meas. & th. / meas. & \\
\hline STF 738 AB & 1 & 0.1675 & 0.070 & $58.2 \%$ & $20.5 \mu \mathrm{rad}$ \\
HJ 3752 AB & 1 & 0.3499 & 0.123 & $64.8 \%$ & $16.3 \mu \mathrm{rad}$ \\
A 2512 & 1 & 0.1355 & 0.112 & $17.3 \%$ & $5.1 \mu \mathrm{rad}$ \\
BU 311 & 1 & 0.6792 & 0.775 & $12.4 \%$ & $2.2 \mu \mathrm{rad}$ \\
\hline
\end{tabular}

Table 8.3: Computed and measured normalized peak values of binary stars.

We can notice that for every star the measured peak of the PSF is smaller than the peak expected through Eq. 8.3 (with the exception of the star BU 311). The amplitude of the PSF decreasing as the field angle increases can be explained by anisoplanatism.

\subsubsection{Problem}

In section 8.4 , we saw that the effect of anisoplanatism can be observed on some of the AO-corrected images. However, the effect could not be observed on all the binary stars measured. This can be explained by the following reasons:

1. The isoplanatic angle is larger than the angular separation between the two binary stars. For example, this is the case of the binary star BU 1052. The two stars are separated by an angle of $2.7 \mu \mathrm{rad}$ and the isoplanatic angle computed from Eq. 4.38 is approximately $16 \mu \mathrm{rad}$.

2. Ideally, the wavefront sensor should have a very narrow FOV in order to receive light only from the reference star. However, in practice the FOV of the wavefront 
sensor is large enough to detect light coming from both binary stars, which affects the centroid location estimate. Therefore, both stars are used as a reference beacon and the AO system does not provide an optimal correction for either of them.

3. The difference in brightness between the two stars plays an important role in the performance of the WFS. If both stars have similar magnitudes, the WFS receives the same amount of light from each star, and not only one of them is used for reference. On the other hand, if star A is much brighter than star B, the WFS receives most of the light from one star, which can be considered of the reference star. In this case, the image of the dimmest star, star B, is affected by anisoplanatism.

\subsection{Deconvolution results}

The images of binary stars are recontructed using the technique presented in chapter 7 and the model for the off-axis PSF presented in chapter 6. The quality of the reconstructed images is measured by computing the MS error between the reconstructed image and the object. The MS error metric was defined in section 7.6.2. Each star is modeled in the object plane as a point source with an amplitude equal to the intensity of the star. The reconstruction error for different stars is given in Table 8.4. First, we can notice that the MS error gets smaller when we reconstruct the image, whether we reconstruct using the model for the off-axis PSF or the on-axis PSF. Second, the reconstruction using the space-varying PSF gives better results than the deconvolution using the on-axis PSF. For example, in the case of the binary star STF 738 AB, we obtain a reconstruction error of $39.3 \%$ using the predicted PSF, as opposed to a $87.8 \%$ using the on-axis PSF. However, for binary stars with a small angular separation, aniso- 
planatism does not have a significant effect. Therefore, the reconstruction results using the predicted PSF or the on-axis PSF are very similar. It is the case of the binary stars BU 311 for which we obtain a $\epsilon_{\text {recons }}$ of $91.2 \%$ compared to a $\epsilon_{\text {recons on-axis }}$ of $91.5 \%$.

\begin{tabular}{|c||c|c|c|}
\hline Star & $\epsilon_{\text {img }}$ & $\epsilon_{\text {recons }}$ & $\epsilon_{\text {recons on-axis }}$ \\
\hline STF 738 AB & $97.2 \%$ & $\mathbf{3 9 . 3} \%$ & $87.8 \%$ \\
STF 611 & $96.3 \%$ & $\mathbf{7 5 . 2} \%$ & $83.4 \%$ \\
A 2512 & $96.0 \%$ & $\mathbf{8 1 . 9} \%$ & $83.9 \%$ \\
BU 311 & $97.8 \%$ & $\mathbf{9 1 . 2} \%$ & $91.5 \%$ \\
HJ 3752 AB & $99.0 \%$ & $\mathbf{9 6 . 1} \%$ & $97.5 \%$ \\
\hline
\end{tabular}

Table 8.4: Reconstruction errors.

Figures 8.4 and 8.5 show the original image and the reconstructed image for binary stars STF 378 AB and STF 611. Figure 8.6 shows the cross-sections of the star and the reconstructed star through the off-axis star.

\subsection{Conclusion}

The binary stars measured at the AEOS facilities had been pre-processed and the effect of anisoplanatism had been observed on some of the data. However, some of the binary stars have an angular separation too small for anisoplanatism to be observed. Also, even though some of the binary stars have an angular separation much larger than the isoplanatic angle, anisoplanatism still could not be observed. This is can be explained by the fact that the wavefront sensor has a FOV larger than the angular separation between the two stars. Therefore, the wavefront sensor receives light from both stars, instead of one reference star.

In conclusion, the effect of anisoplanatism on measured data can be observed the best on binary stars that respect the following conditions: first, the angular separation 

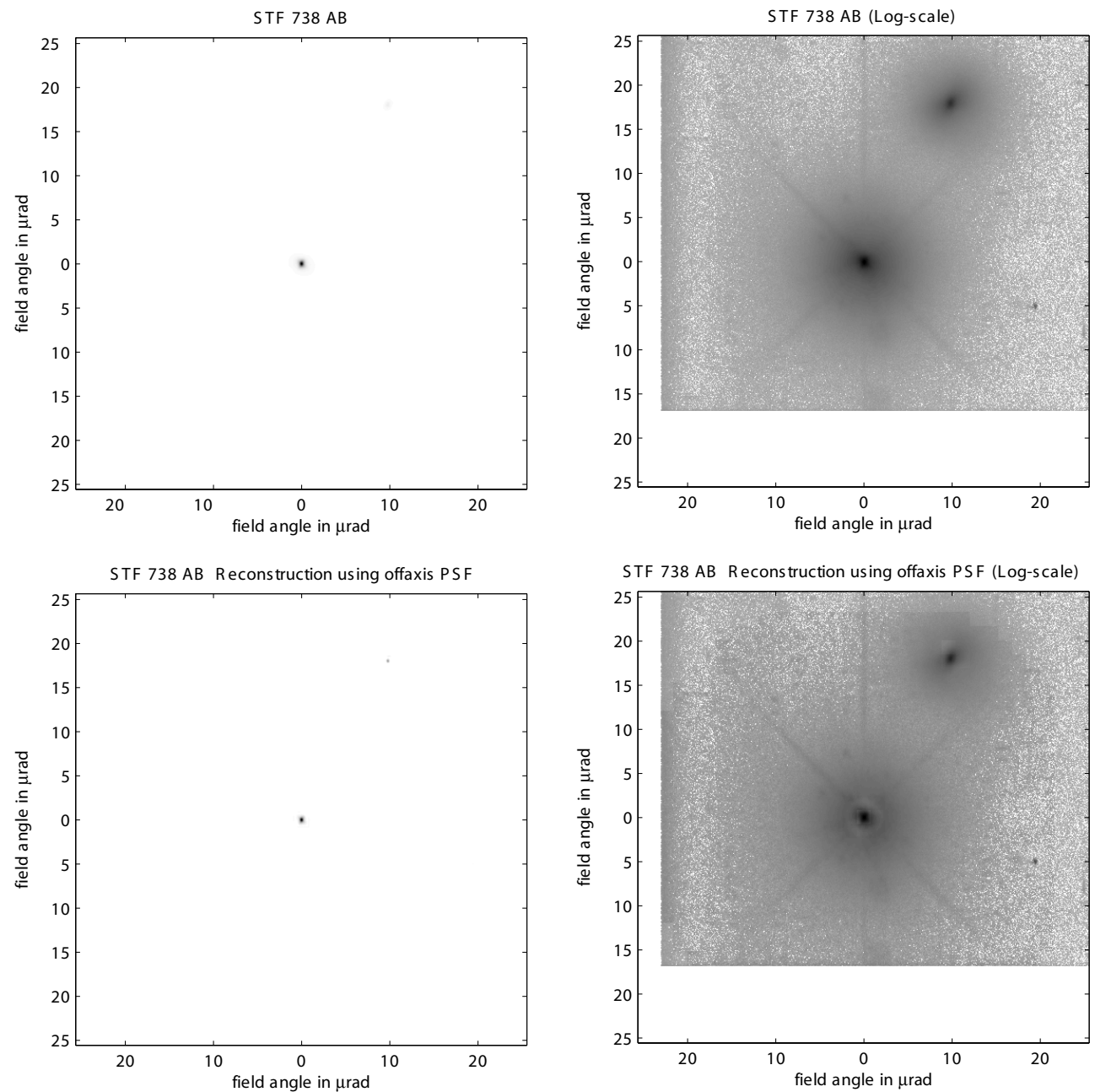

Figure 8.4: Measured and reconstructed image for binary star STF 738 AB. 

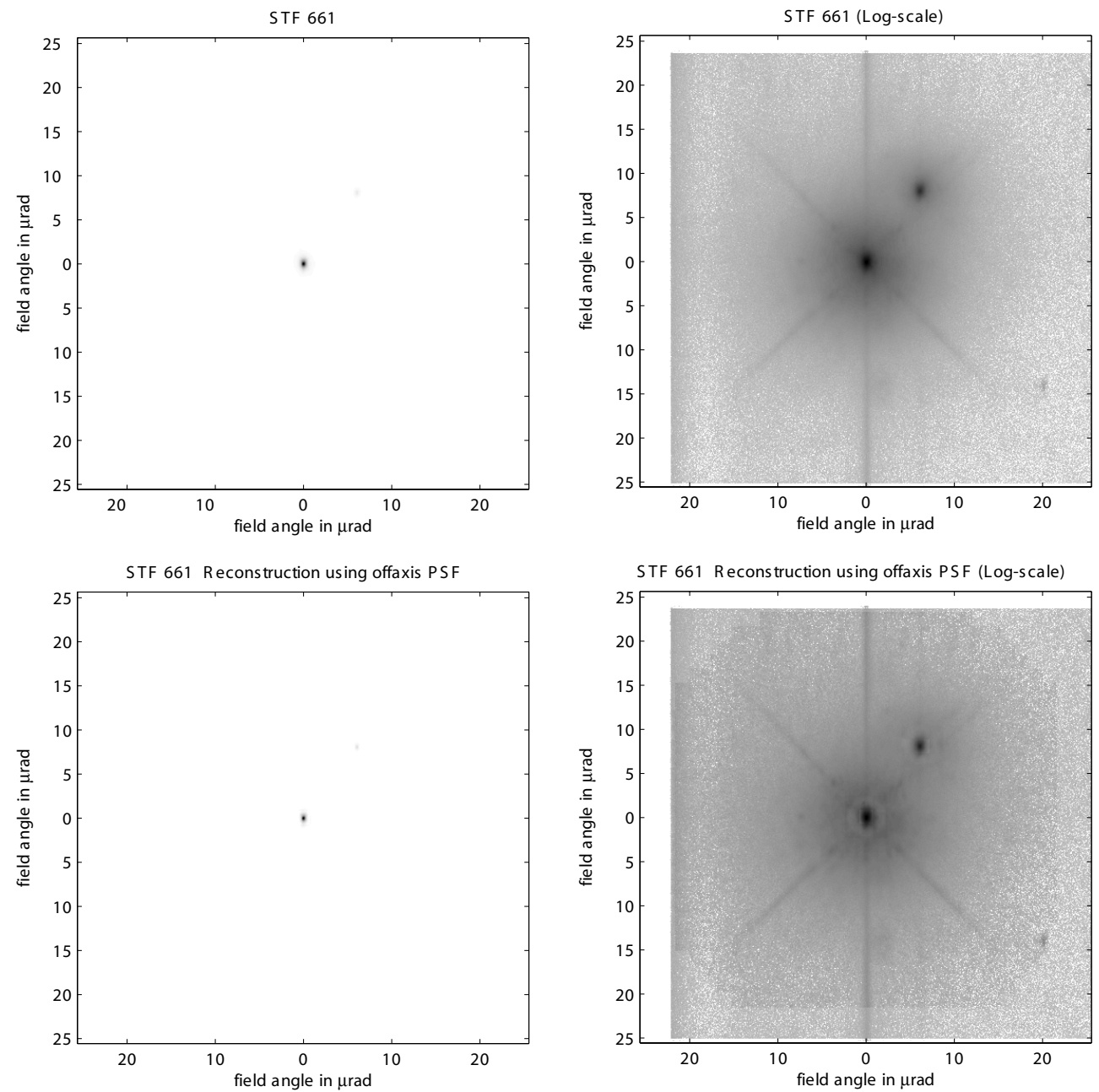

Figure 8.5: Measured and reconstructed image for binary star STF 611. 


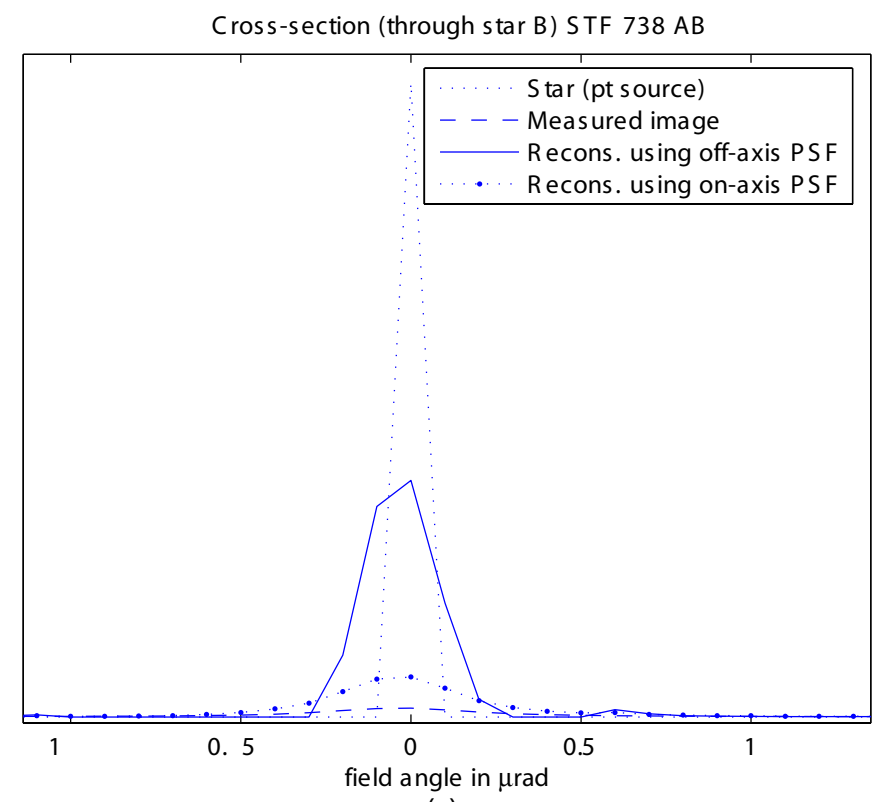

(a)

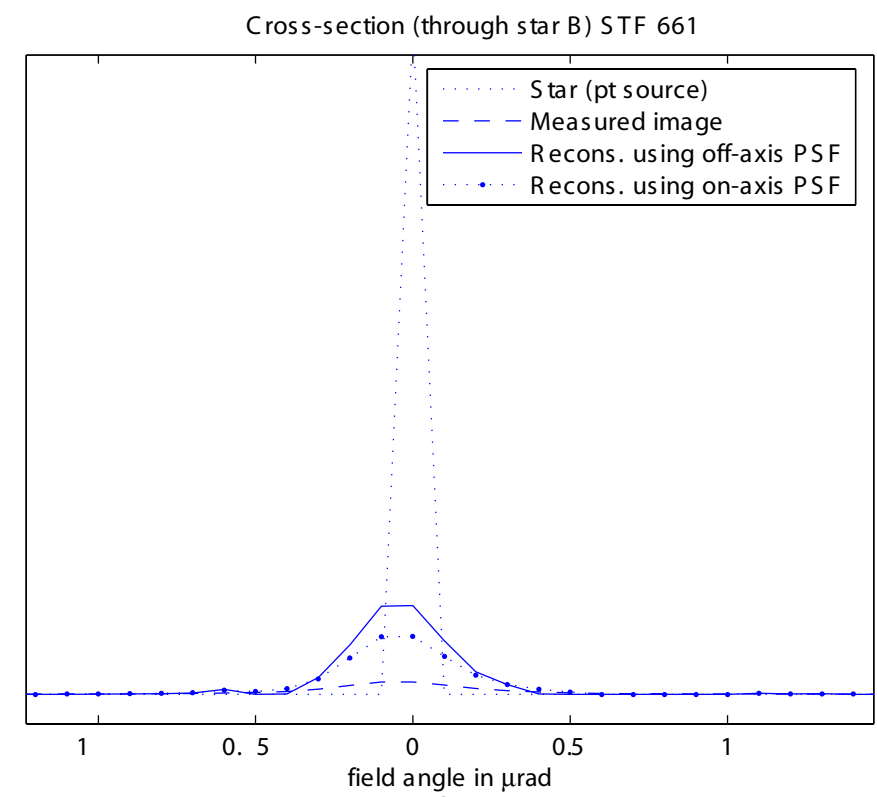

(b)

Figure 8.6: Cross-sections of the measured and reconstructed stars: (a) STF 738 AB, and (b) STF 611. 
needs to be larger than the isoplanatic angle approximately. Second, the star used as a reference needs to be much brighter than the secondary one.

The measured images had been reconstructed with the technique described in chapter 7. The deconvolution results show an improvement of the MS error between the reconstructed image and the model for the star. Also, the deconvolution using the predicted PSF gives better results than the deconvolution using the on-axis PSF. 


\section{CHAPTER 9}

\section{Conclusion}

\subsection{Summary of results}

In this dissertation, we presented a method to reconstruct anisoplanatic adaptive optics images. We now summarize the results of the dissertation.

After posing the problem of imaging using adaptive optics under anisoplanatic conditions, we gave a background on wave optics. The science of imaging through turbulence was then presented, as well as models for atmospheric turbulence. AO systems were described in detail, and models for each of their components were given. My research begins in chapter 5 where I described the wave propagation simulation I used. The simulation included a model for the atmosphere as well as for the AO imaging system. The simulation allowed us to obtain long exposure PSF as well as LE intensity images of a stellar field through the atmosphere. The effect of anisoplanatism could be observed on the simulated images. To reconstruct those LE AO-corrected images, the knowledge of the space-varying PSF is essential.

\section{Prediction of the off-axis point spread function}

In chapter 6, a method for prediction of the LE AO-corrected off-axis PSF as a continuous function of the field angle was presented. A model for the PSF was introduced, 
allowing to parameterize the PSF with a small number of coefficients. The methods shows a prediction error varying from $0.9 \%$ to $2.7 \%$ depending on the field angle considered and the seeing conditions.

\section{Reconstruction of anisoplanatic adaptive optics images}

The method for prediction of the off-axis PSF presented in chapter 6 is validated in chapter 7. In chapter 7, the predicted PSF was used for the deconvolution of anisoplanatic images. The reconstruction technique is applied to simulated images, as well as experimental data. The deconvolution results using the predicted off-axis PSF are compared to the deconvolution results using the on-axis PSF, which would be the type of deconvolution used under isoplanatic conditions. The reconstruction results were presented and the technique showed an improvement of the original image, for simulated and experimental data.

\section{Results for simulated images}

In the case of simulated anisoplanatic star field images, two commonly used image restoration techniques were considered: the Tikhonov regularization and the EM algorithm. The predicted PSF was showed to give MS errors between the reconstructed image and the object $7.2 \%$ to $84.8 \%$ smaller than the on-axis PSF.

The influence of the SNR was also studied. Our study shows that a SNR larger than 100 and good seeing conditions $\left(r_{0}\right.$ larger $20 \mathrm{~cm}$ ), the inverse problem does not need regularization (Tikhonov coefficient $\alpha=0$ ). However, under different conditions, a regularization of the inverse problem is necessary.

The performance of the reconstruction decreases as the seeing conditions degrade or as the SNR gets smaller. Both reconstruction techniques behave differently depending on 
the seeing conditions and the SNR. The best result ( $84.8 \%$ of improvement from the on-axis PSF deconvolution) is obtained using the Tikhonov regularization, with a SNR of $10^{4}$ and a Fried parameter of $25 \mathrm{~cm}$. The EM algorithm gives better results than the Tikohonov regularization for a low SNR (smaller than 100). For example, for a SNR of 0.1 and a Fried parameter of $10 \mathrm{~cm}$, we obtain an improvement factor of $64.2 \%$ using the EM algorithm, as opposed to $7.2 \%$ using the Tikhonov regularization.

\section{Results for experimental data}

Binary stars were measured at the AEOS facilities. In some case, anisoplanatism could not be observed in the images. It was explained by the fact that the wavefront sensor has a FOV larger than the angular separation between the two stars. Therefore, the wavefront sensor receives light from both stars, instead of one reference star. The effect of anisoplanatism on measured data can be observed the best on binary stars that respect the following conditions: first, the angular separation needs to be larger than the isoplanatic angle approximately. Second, the star used as a reference needs to be much brighter than the secondary one.

The images obtained were reconstructed using the technique presented in chapter 6 . The reconstruction results using the predicted PSF technique shows an improvement of the MS error between the reconstructed image and the object up to $55.2 \%$.

\subsection{Future work}

\section{Extension of the method: interpolation in term of $r_{0}$}

We saw in chapter 6 that the PSF depends on the field angle $\theta$, but it also depends on the Fried parameter $r_{0}$. Therefore the coefficients $a_{i}$ 's and $b_{i}$ 's of the parameterized 
model for the PSF depend on $\theta$ and $r_{0}$ as well. For a fixed $\theta$, the $a_{i}$ 's and $b_{i}$ 's are functions of $r_{0}$ and can be denoted respectively $a_{i}\left(r_{0}\right)$ and $b_{i}\left(r_{0}\right)$. The previous method of interpolation of the $a_{i}(\theta)$ and $b_{i}(\theta)$ in term of the field angle $\theta$ can then be extended to the interpolation of the $a_{i}\left(r_{0}\right)$ and $b_{i}\left(r_{0}\right)$ in term of $r_{0}$.

Our goal in this dissertation is to restore anisoplanatic AO-corrected images. Therefore, the knowledge of the PSF as a function of the field angle represents an interest. However, the prediction of the PSF as a function of the Fried parameter $r_{0}$ seems interesting and could be the object of further research. 


\section{APPENDIX A}

Results: Prediction of the PSF 


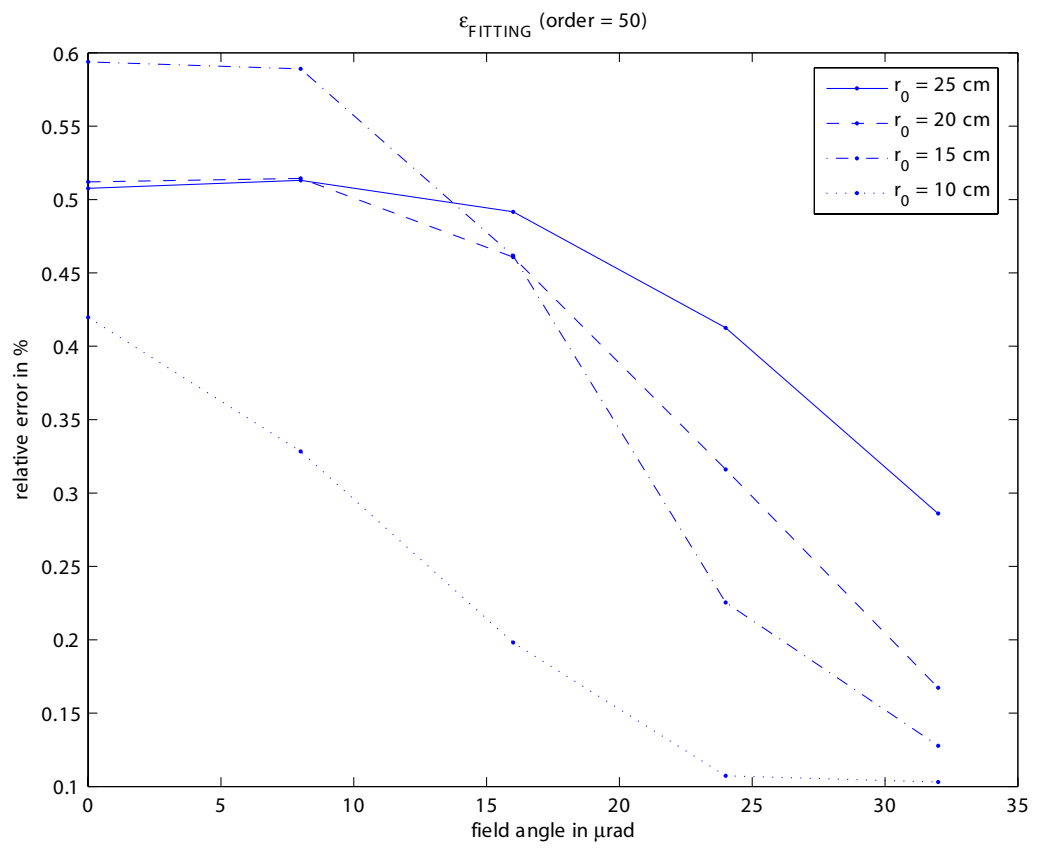

Figure 1.1: Fitting error $\epsilon_{\text {FITTING }}$ vs. field angle. 

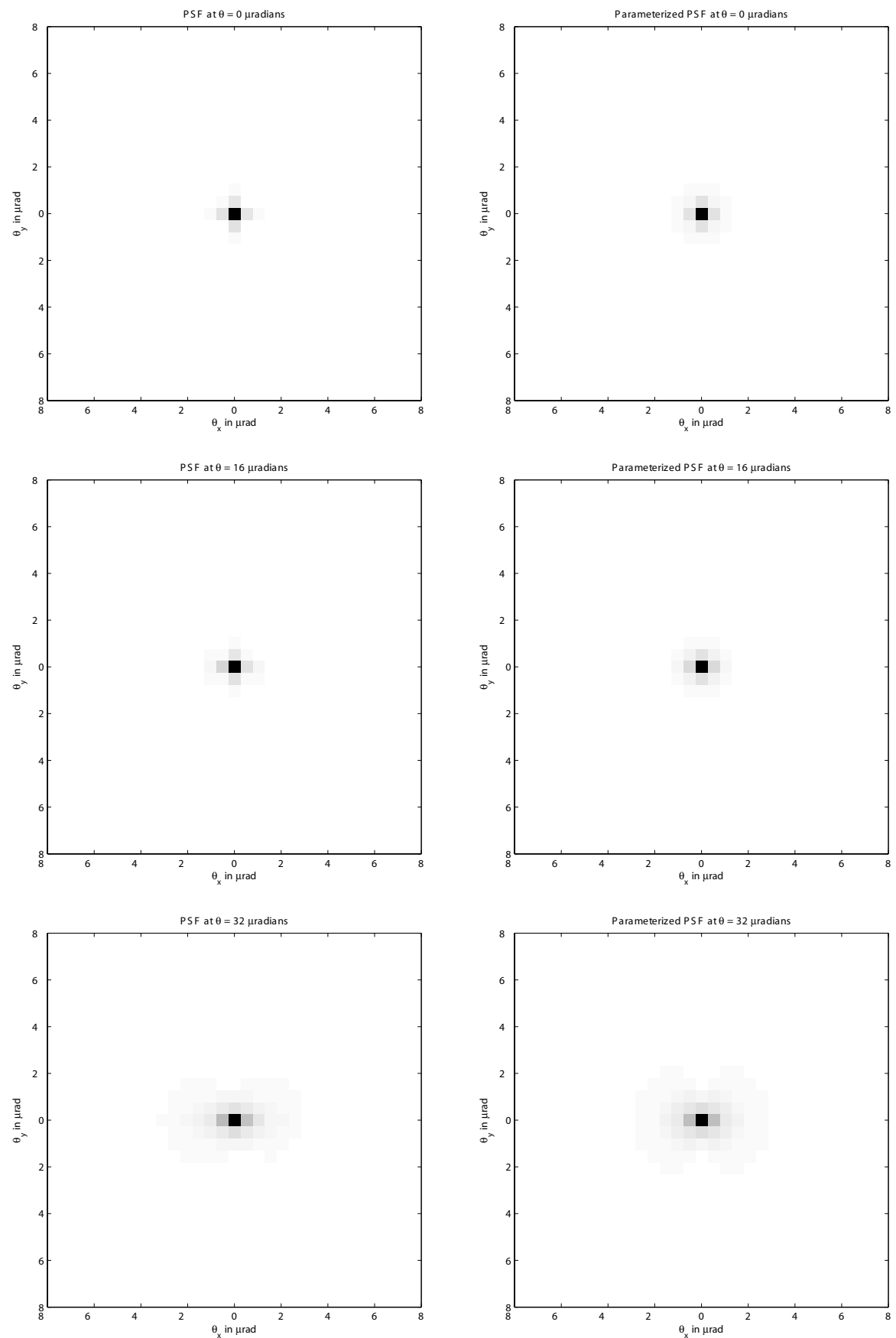

Figure 1.2: Parameterized and simulated PSF's for $\theta=0,16$, and 32 $\mu$ radians, and $r_{0}=25 \mathrm{~cm}$. 

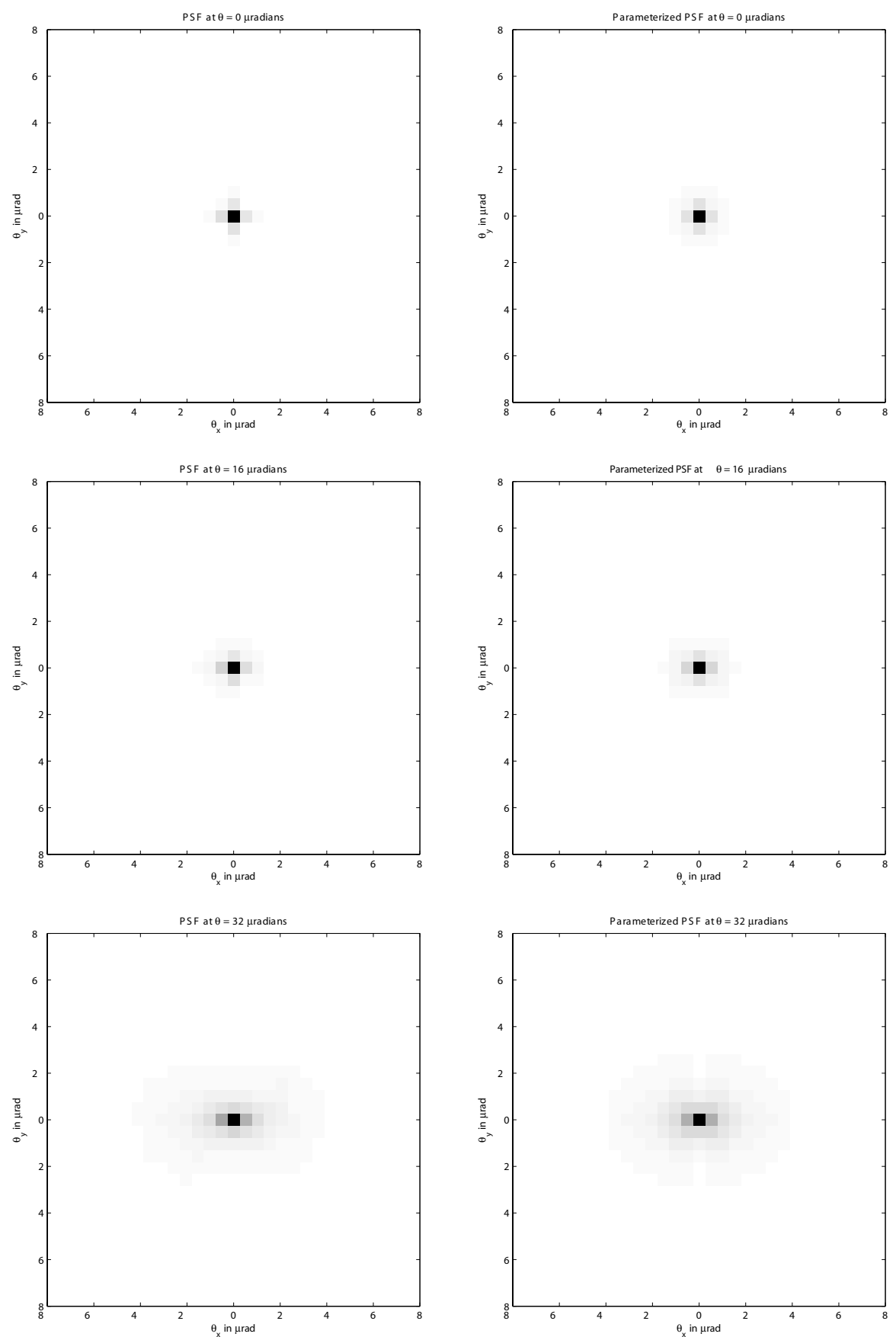

Figure 1.3: Parameterized and simulated PSF's for $\theta=0,16$, and 32 rradians, and $r_{0}=20 \mathrm{~cm}$. 

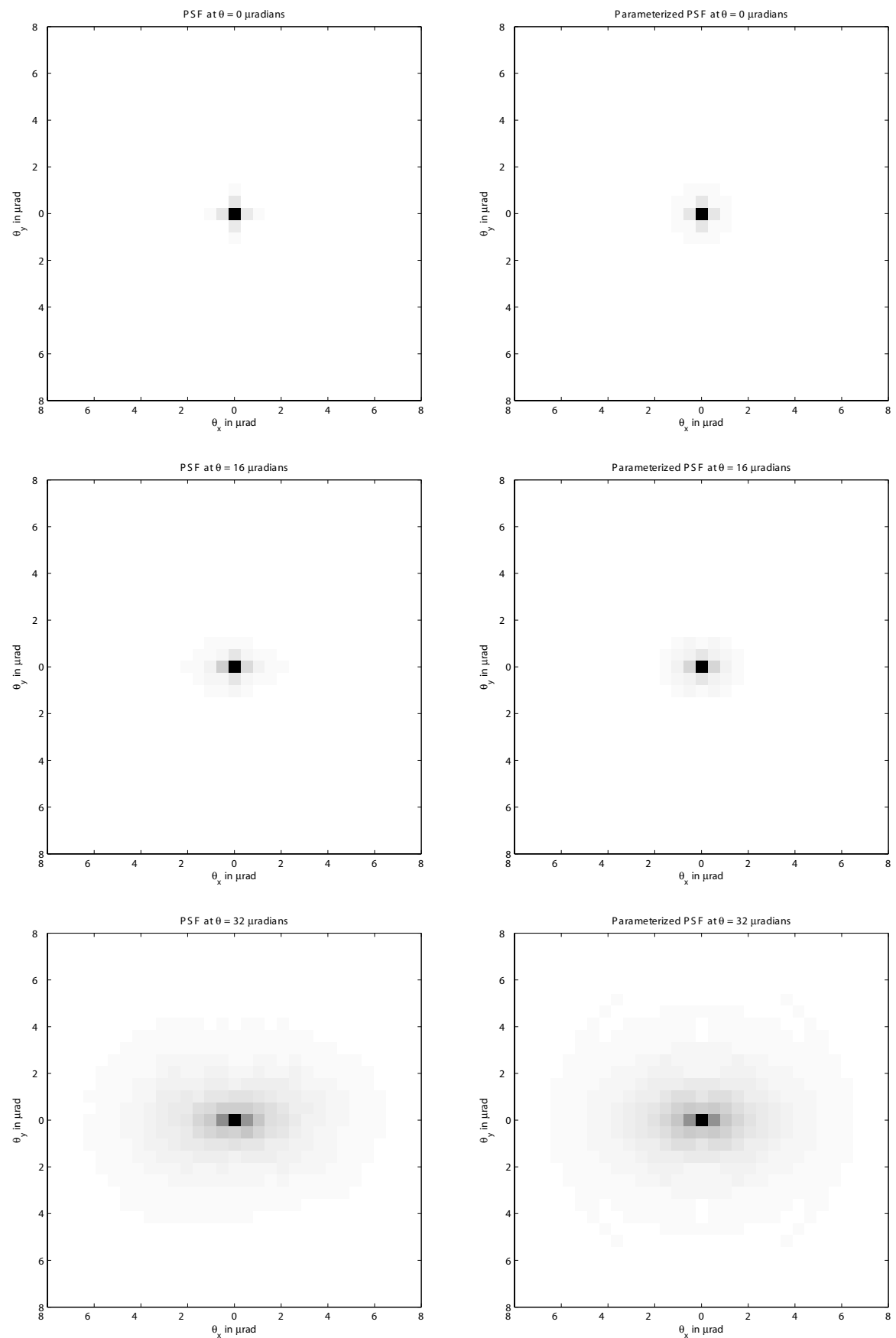

Figure 1.4: Parameterized and simulated PSF's for $\theta=0,16$, and 32 $\mu$ radians, and $r_{0}=15 \mathrm{~cm}$. 

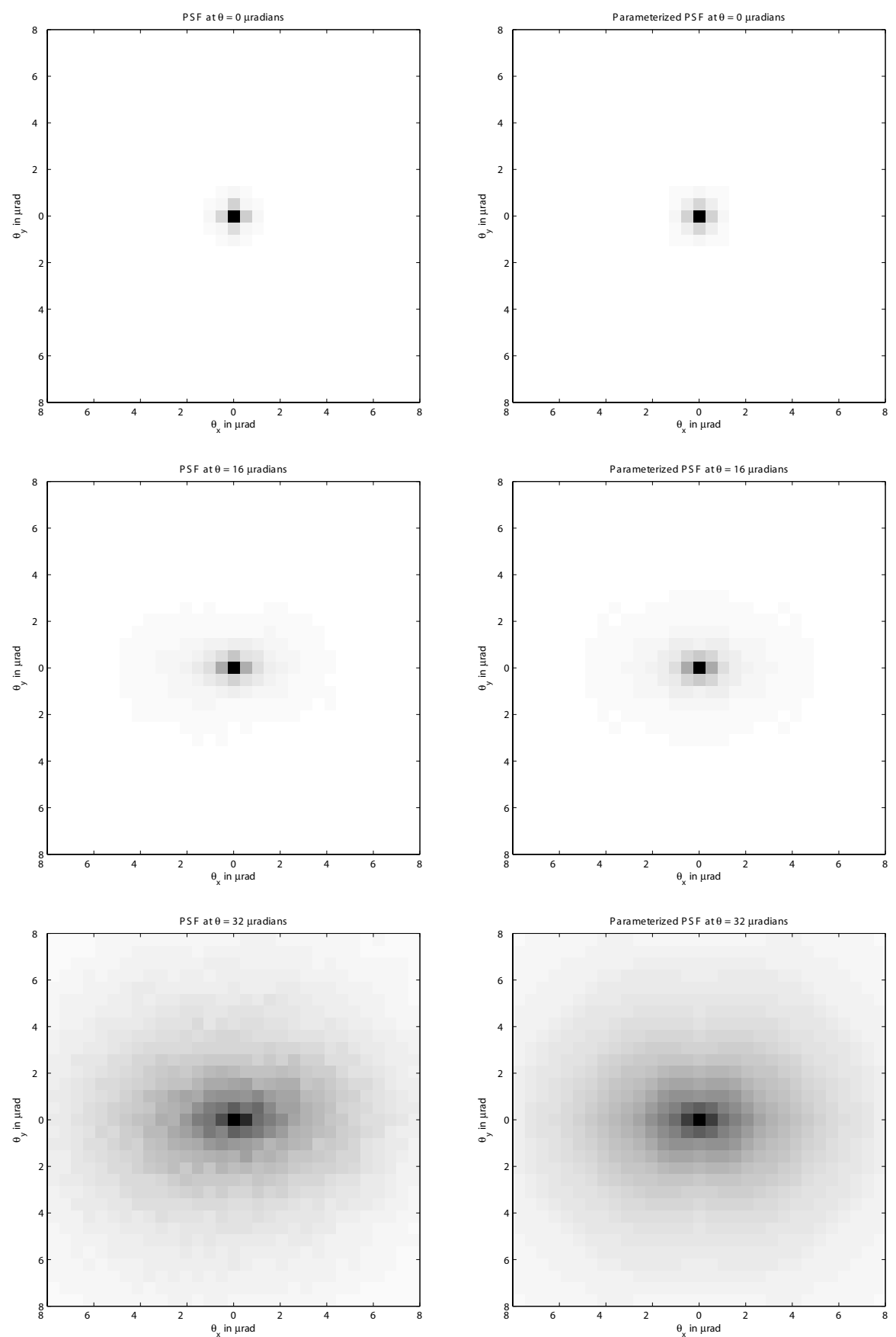

Figure 1.5: Parameterized and simulated PSF's for $\theta=0,16$, and 32 $\mu$ radians, and $r_{0}=10 \mathrm{~cm}$. 

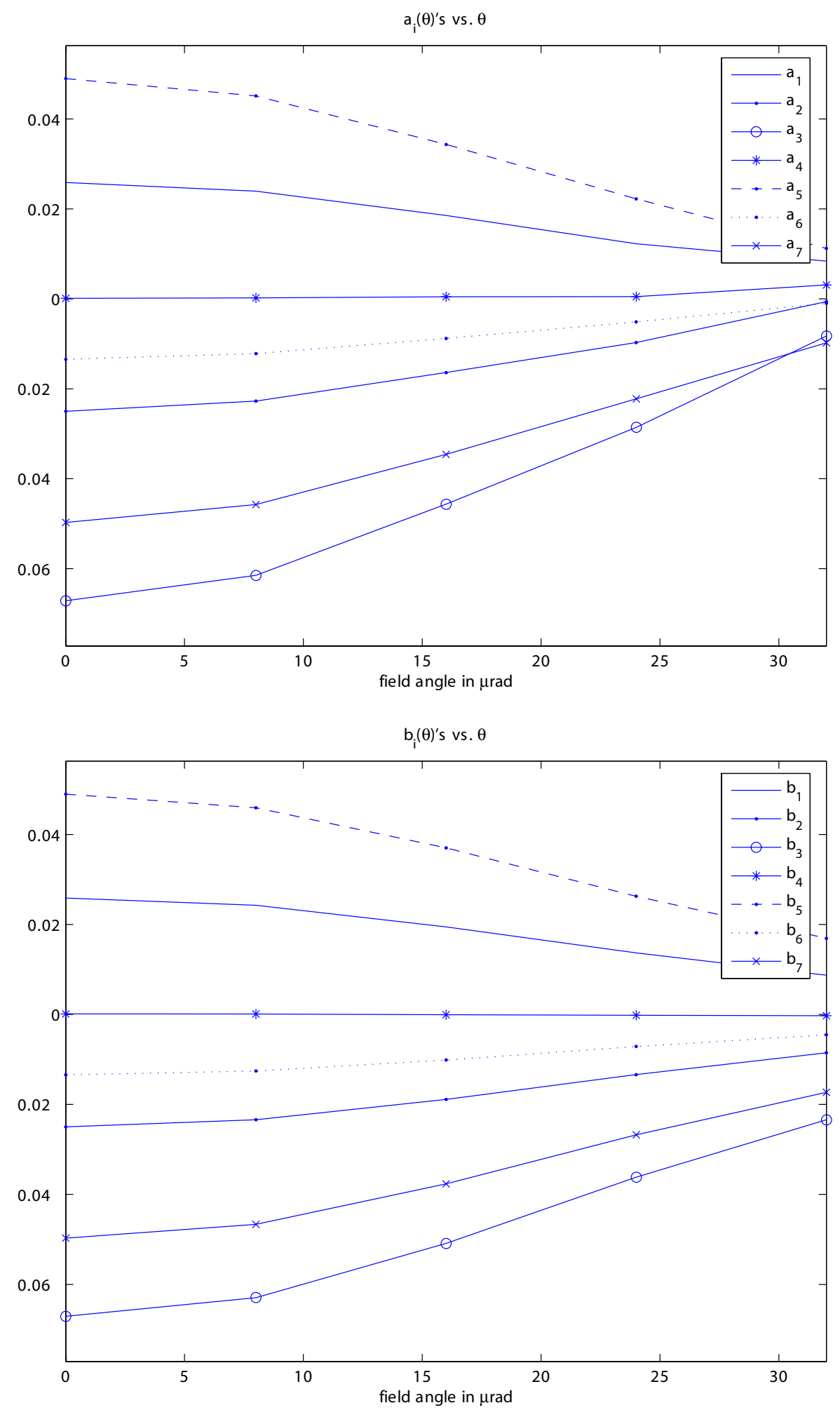

Figure 1.6: Coefficients $a_{i}$ 's and $b_{i}$ 's vs. field angle for $r_{0}=25 \mathrm{~cm}$. 

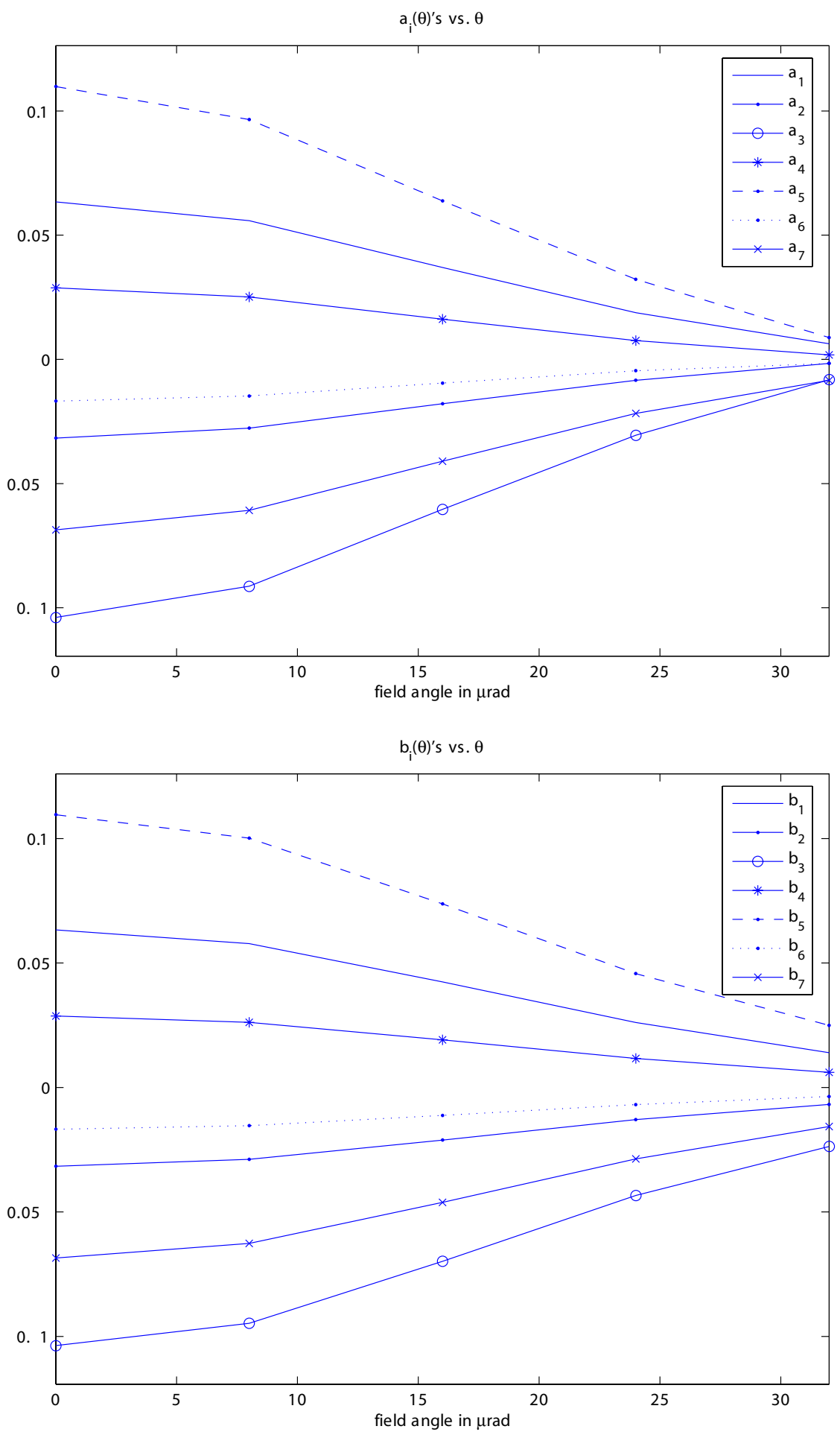

Figure 1.7: Coefficients $a_{i}$ 's and $b_{i}$ 's vs. field angle for $r_{0}=20 \mathrm{~cm}$. 

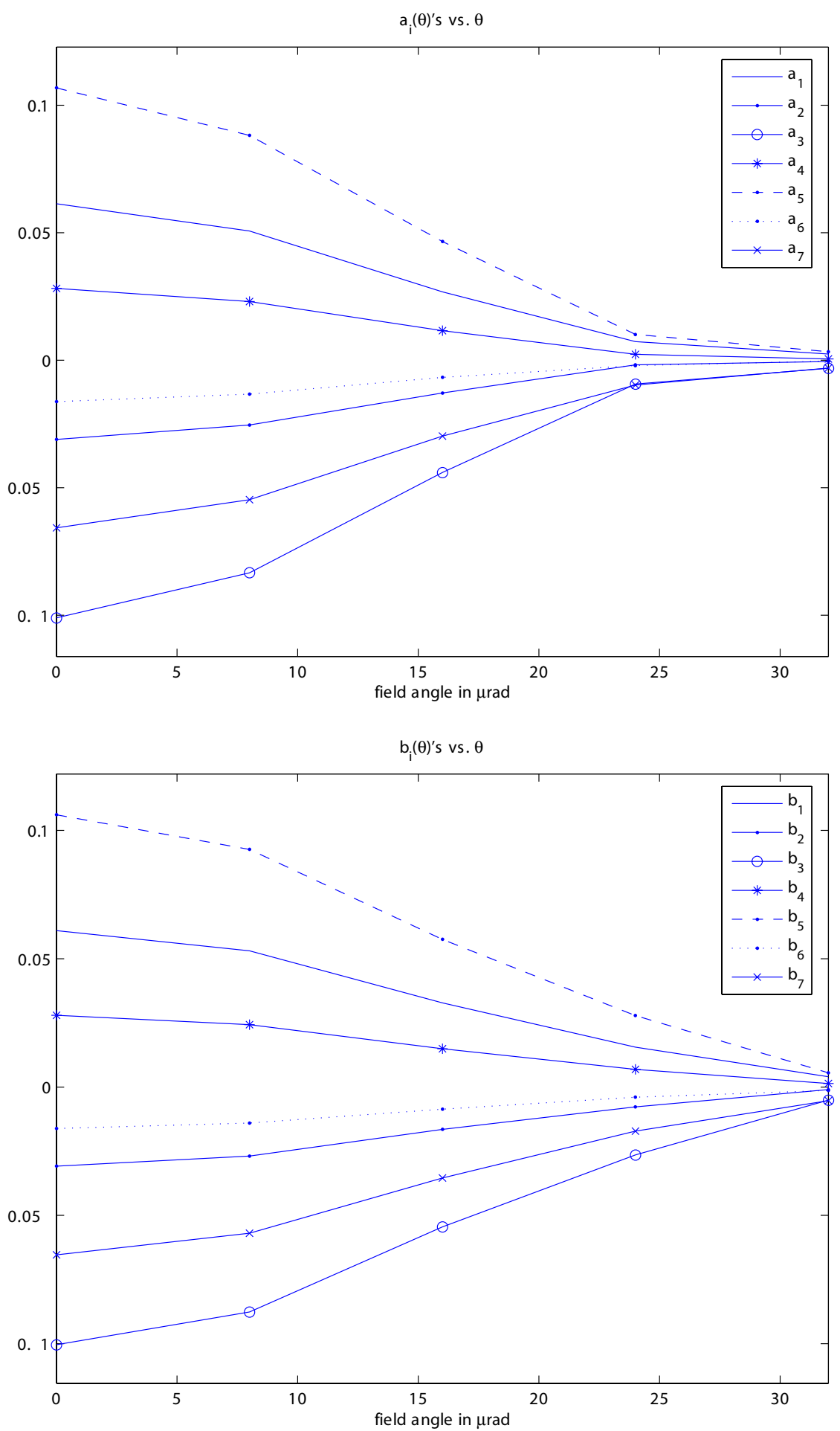

Figure 1.8: Coefficients $a_{i}$ 's and $b_{i}$ 's vs. field angle for $r_{0}=15 \mathrm{~cm}$. 

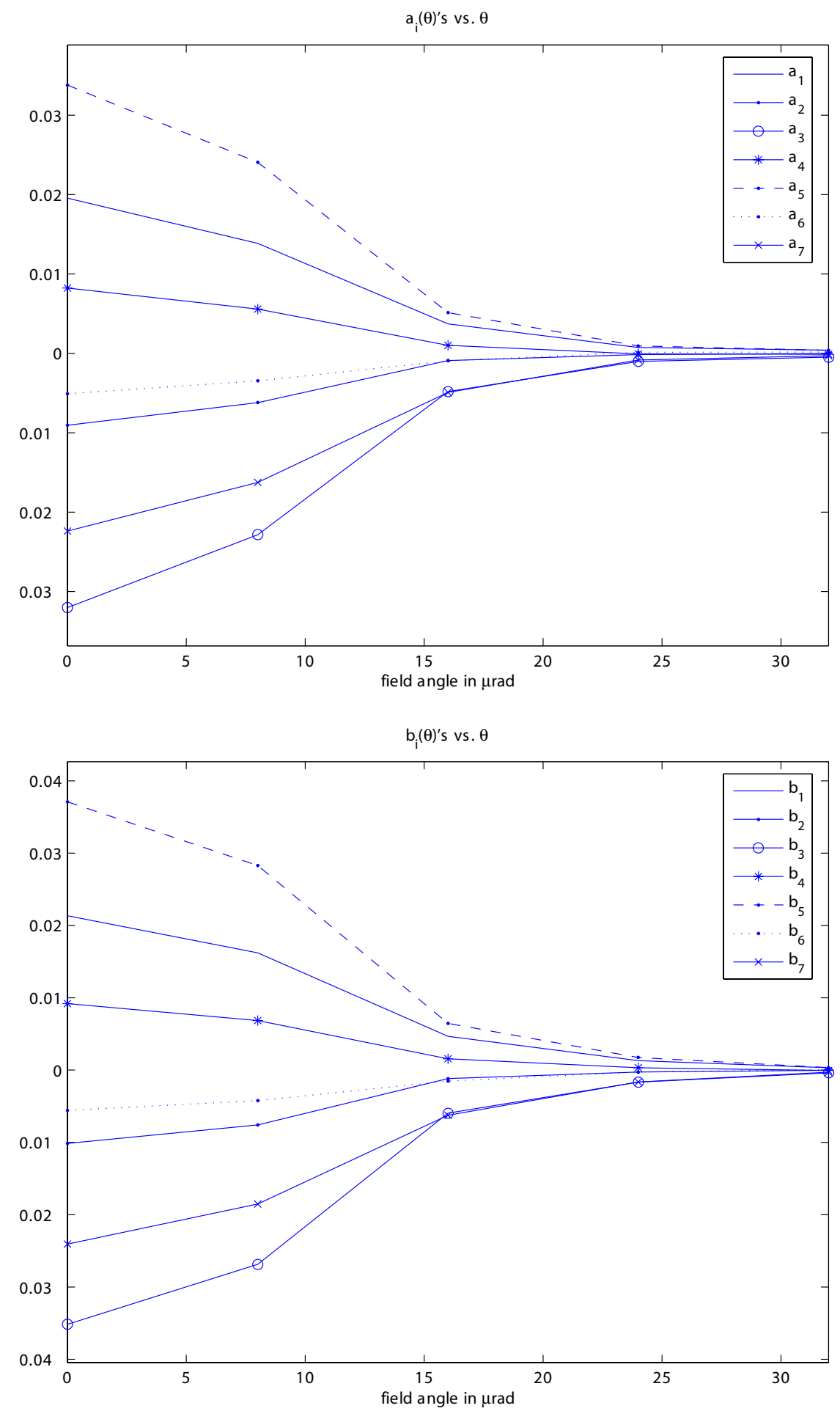

Figure 1.9: Coefficients $a_{i}$ 's and $b_{i}$ 's vs. field angle for $r_{0}=10 \mathrm{~cm}$. 


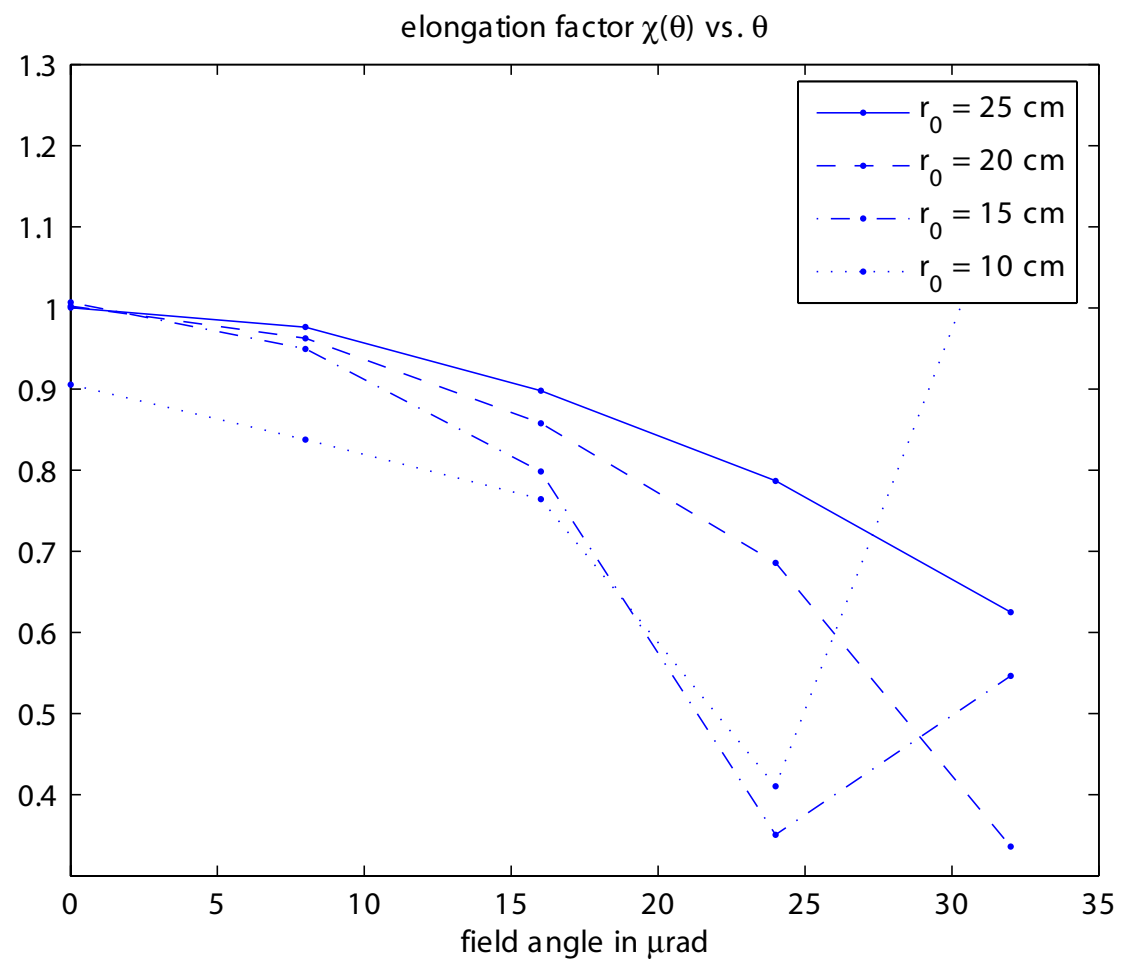

Figure 1.10: Elongation factor $\chi(\theta)$ vs. $\theta$ for different $r_{0}$. 

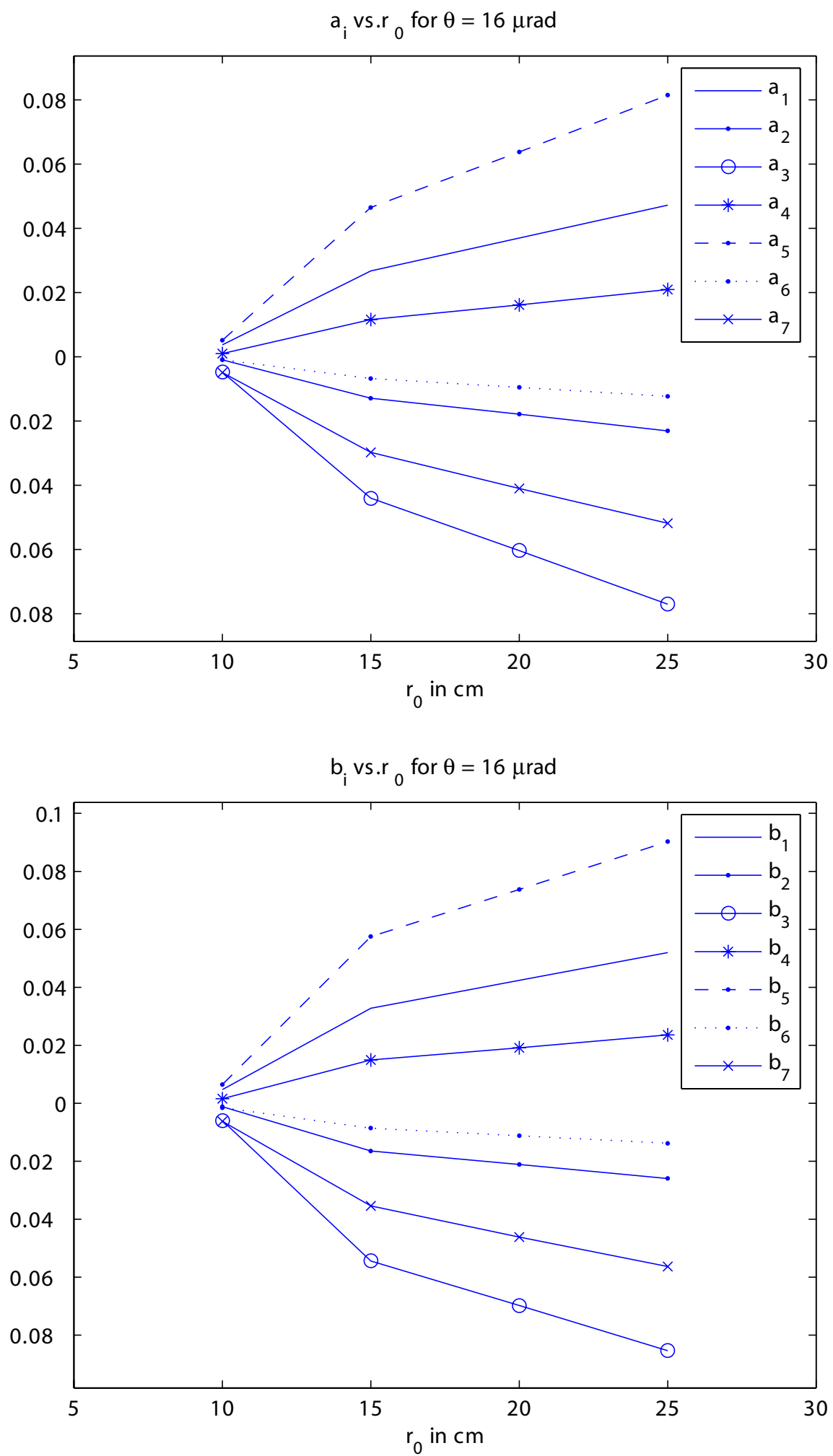

Figure 1.11: Coefficients $a_{i}$ 's and $b_{i}$ 's vs. $r_{0}$ for $\theta=16 \mu \mathrm{rad}$. 

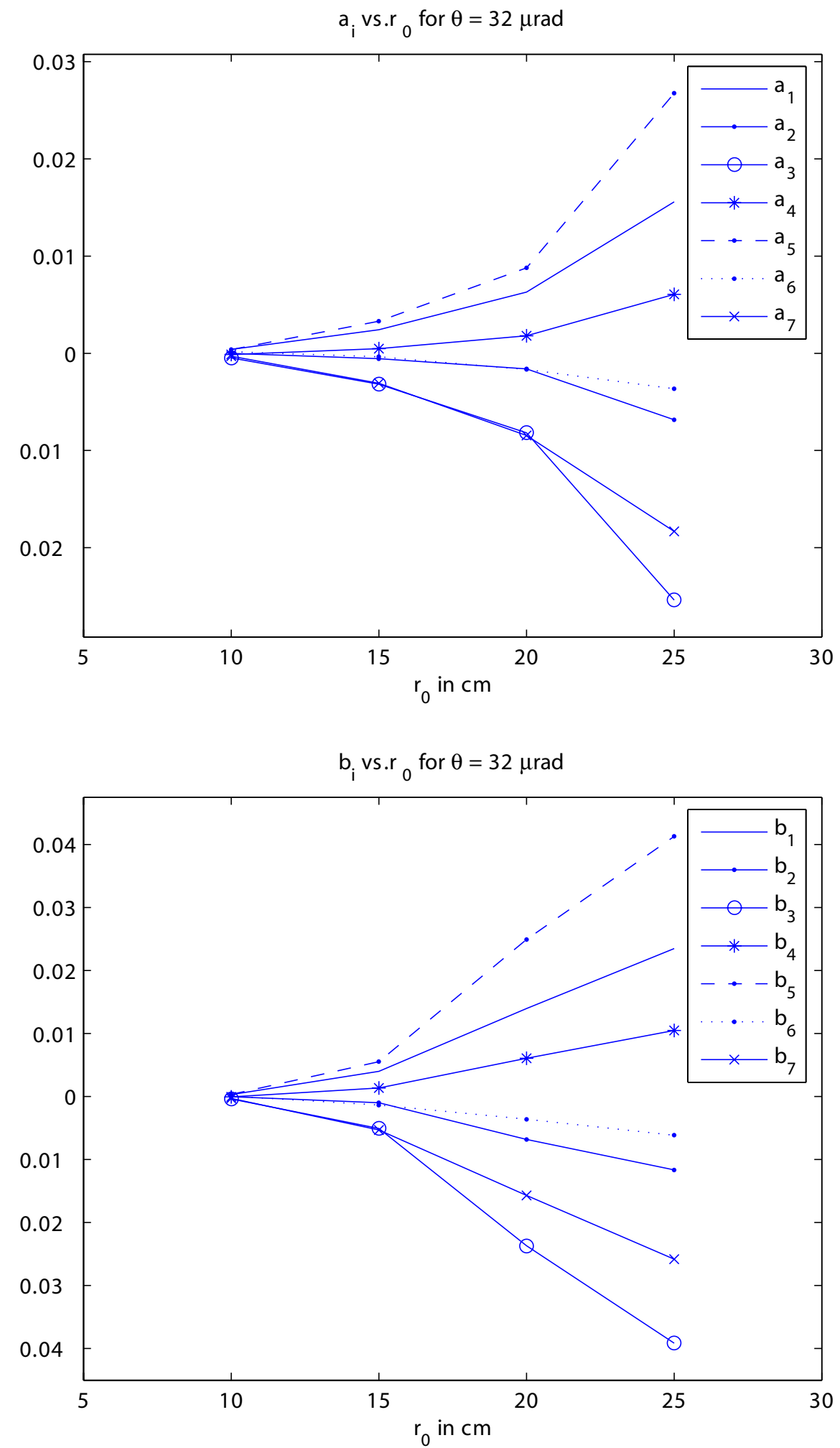

Figure 1.12: Coefficients $a_{i}$ 's and $b_{i}$ 's vs. $r_{0}$ for $\theta=32 \mu \mathrm{rad}$. 


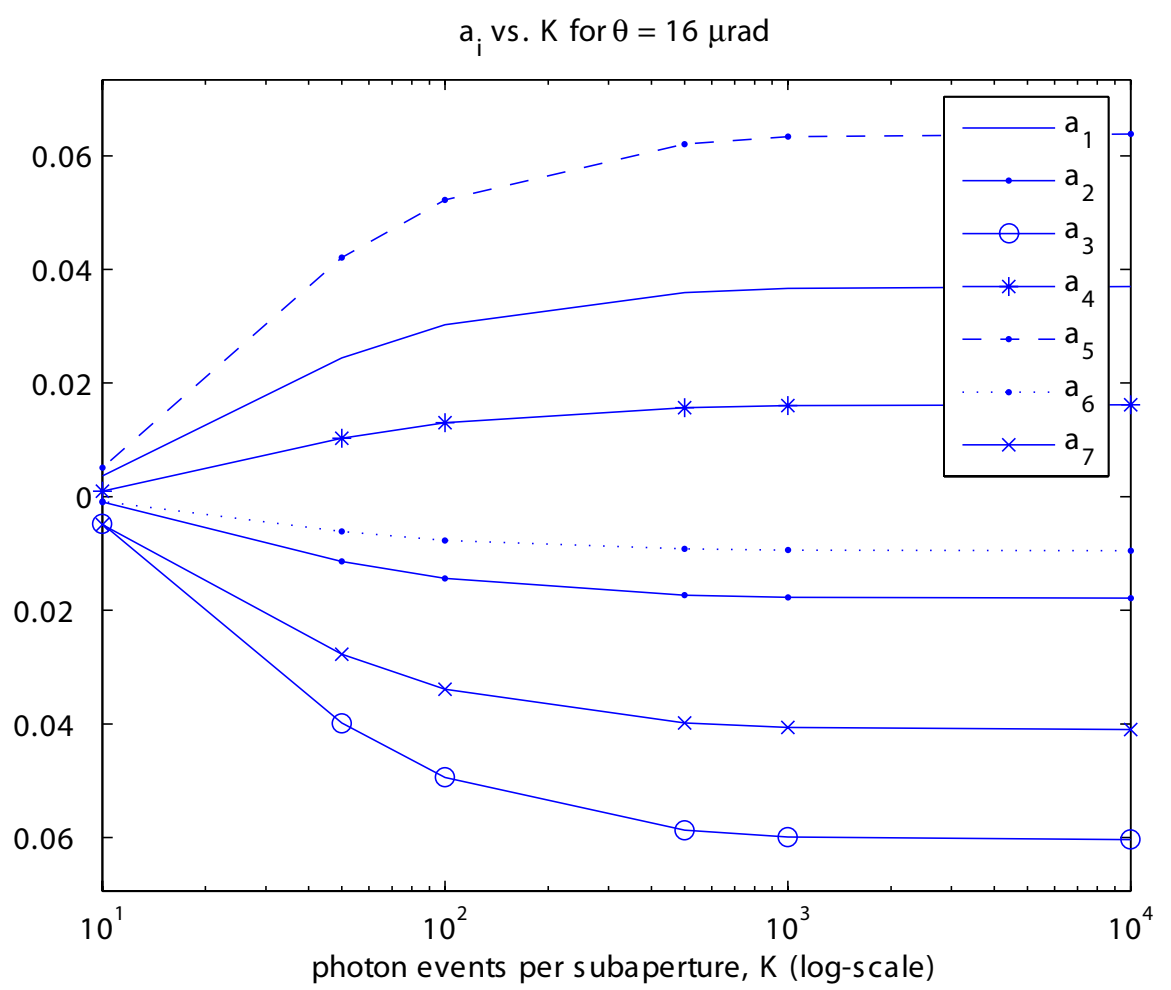

$b_{i}$ vs. $K$ for $\theta=16 \mu \mathrm{rad}$

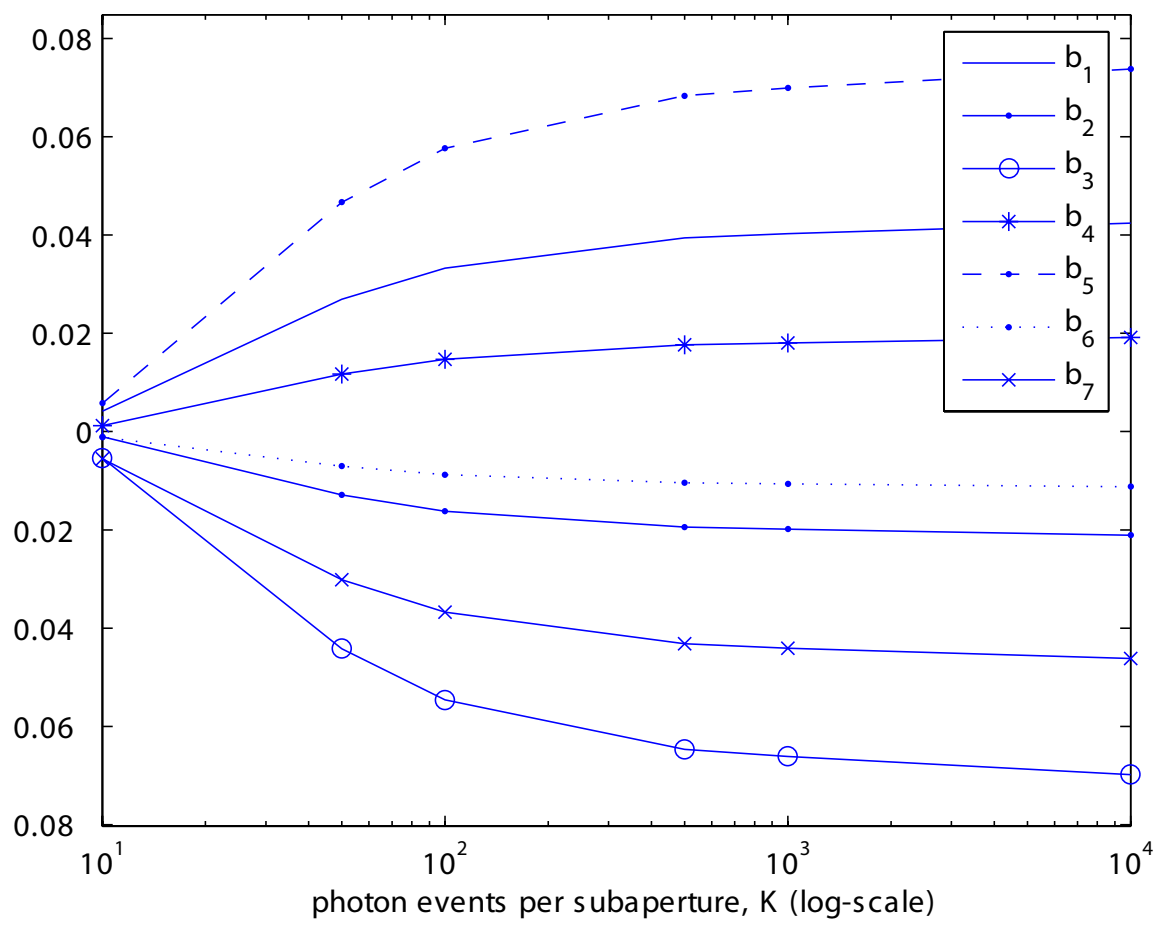

Figure 1.13: Coefficients $a_{i}$ 's and $b_{i}$ 's vs. $\bar{K}$ for $\theta=16 \mu$ rad. 

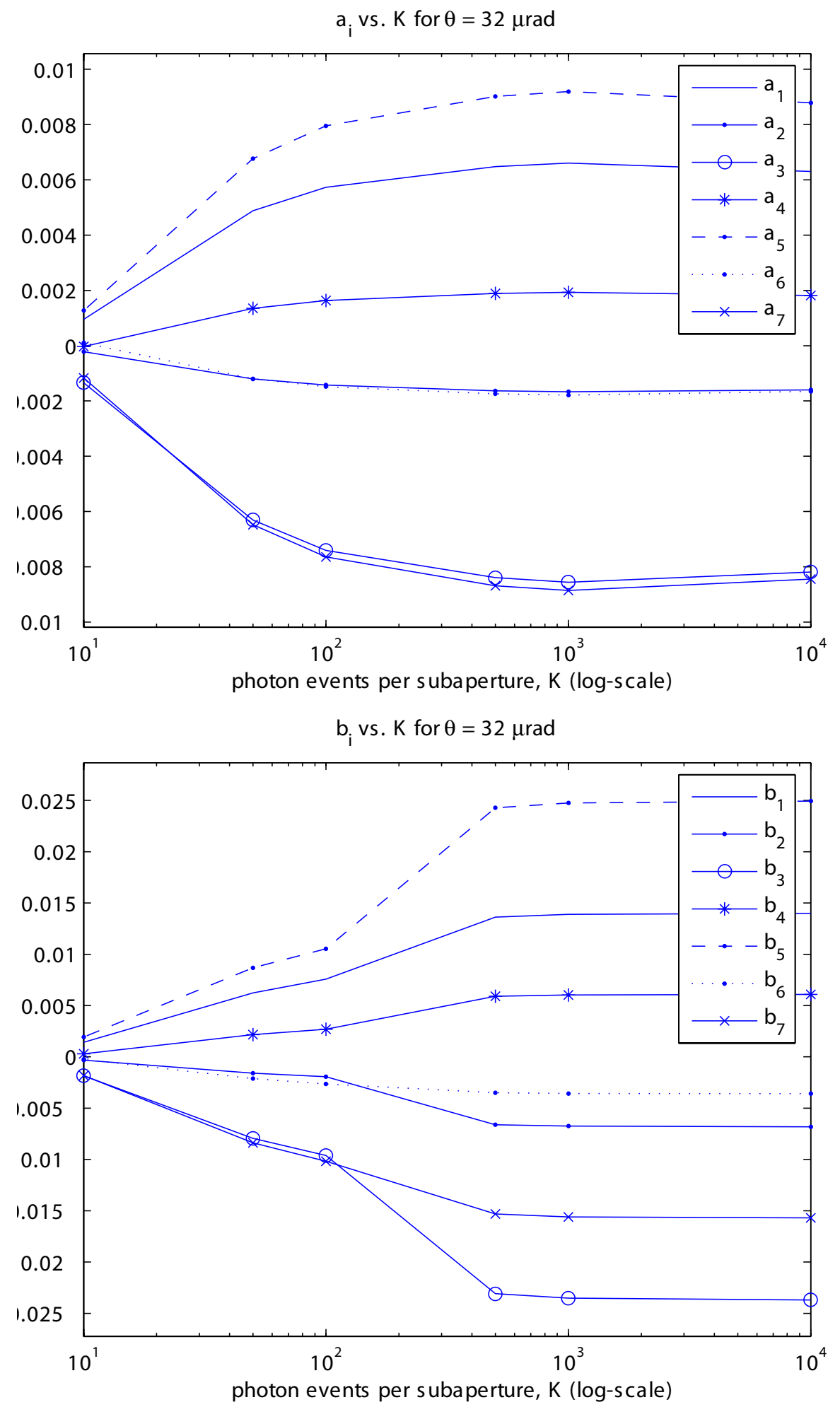

Figure 1.14: Coefficients $a_{i}$ 's and $b_{i}$ 's vs. $\bar{K}$ for $\theta=32 \mu$ rad. 

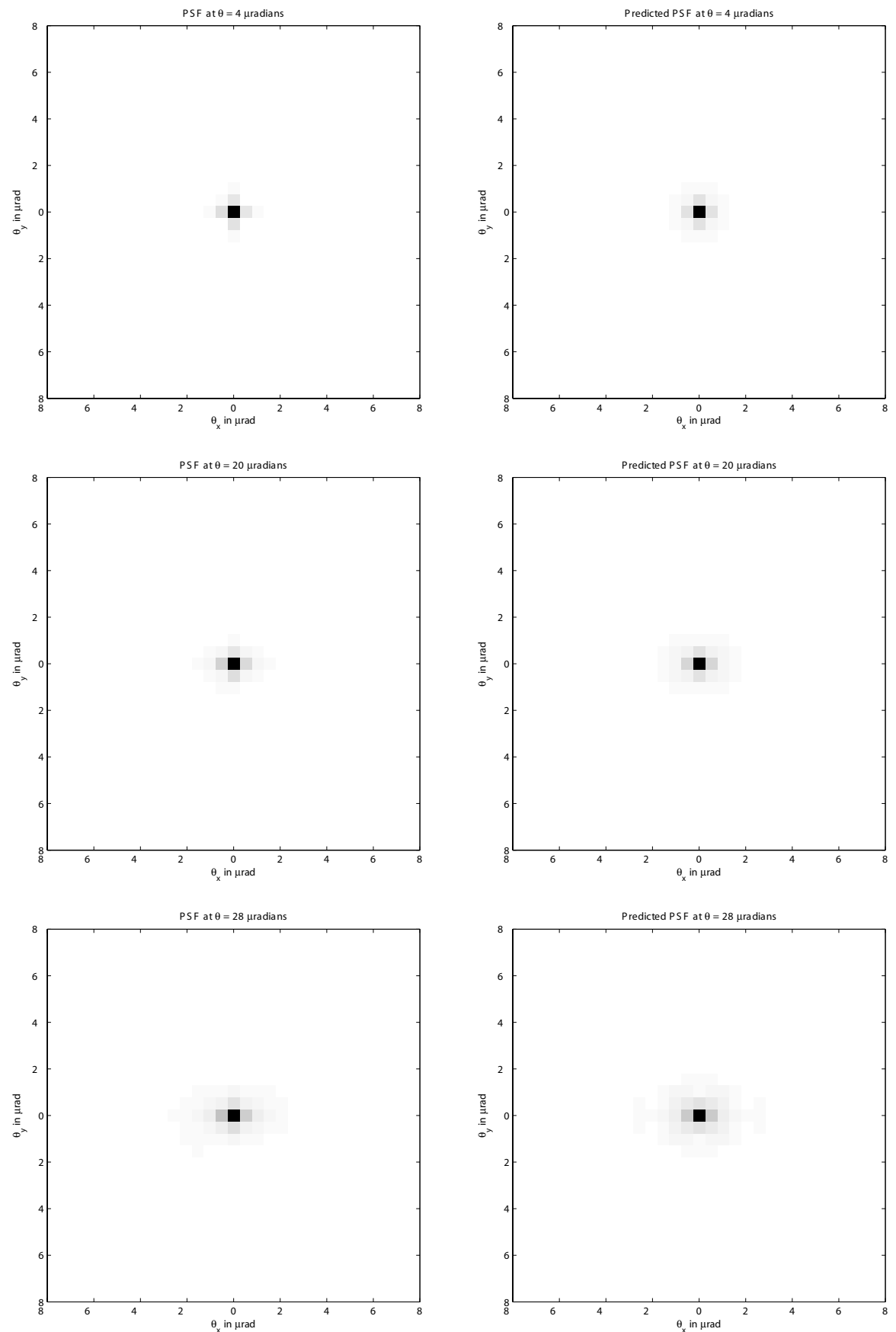

Figure 1.15: Predicted and simulated PSF's for $\theta=4$, 20, and 28 uradians, and $r_{0}=$ $25 \mathrm{~cm}$. 

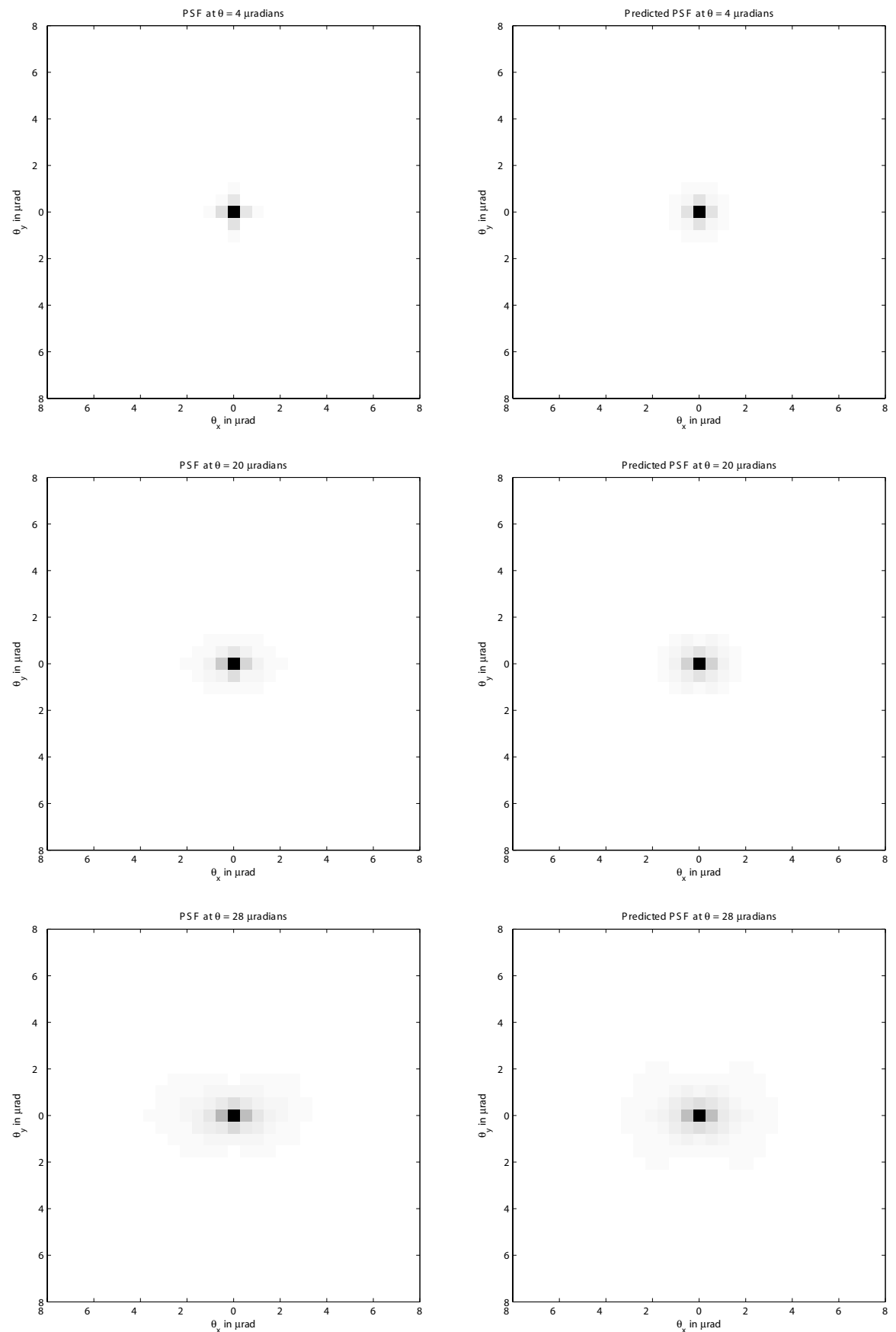

Figure 1.16: Predicted and simulated PSF's for $\theta=4$, 20, and 28 pradians, and $r_{0}=$ $20 \mathrm{~cm}$. 

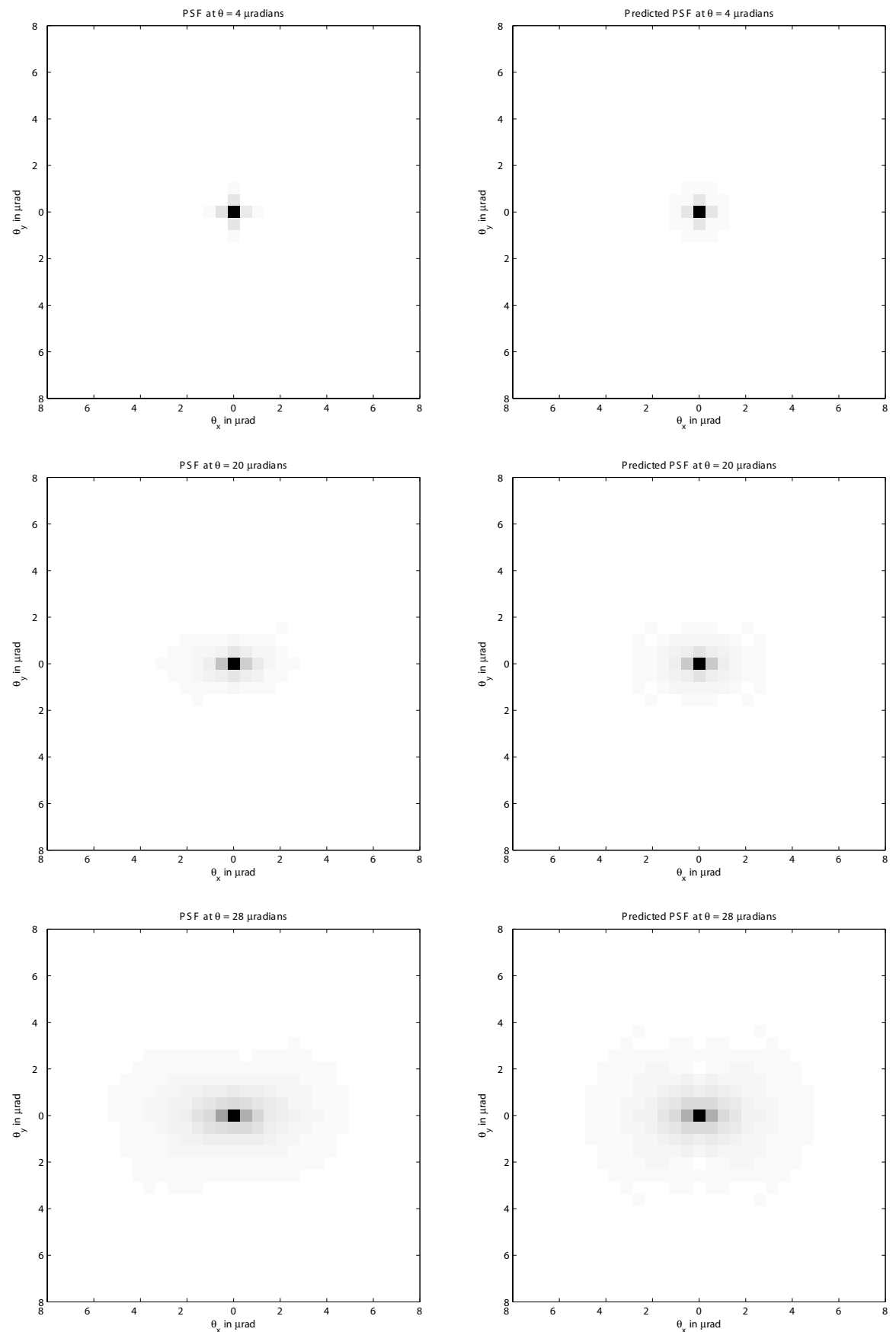

Figure 1.17: Predicted and simulated PSF's for $\theta=4$, 20, and 28 uradians, and $r_{0}=$ $15 \mathrm{~cm}$. 

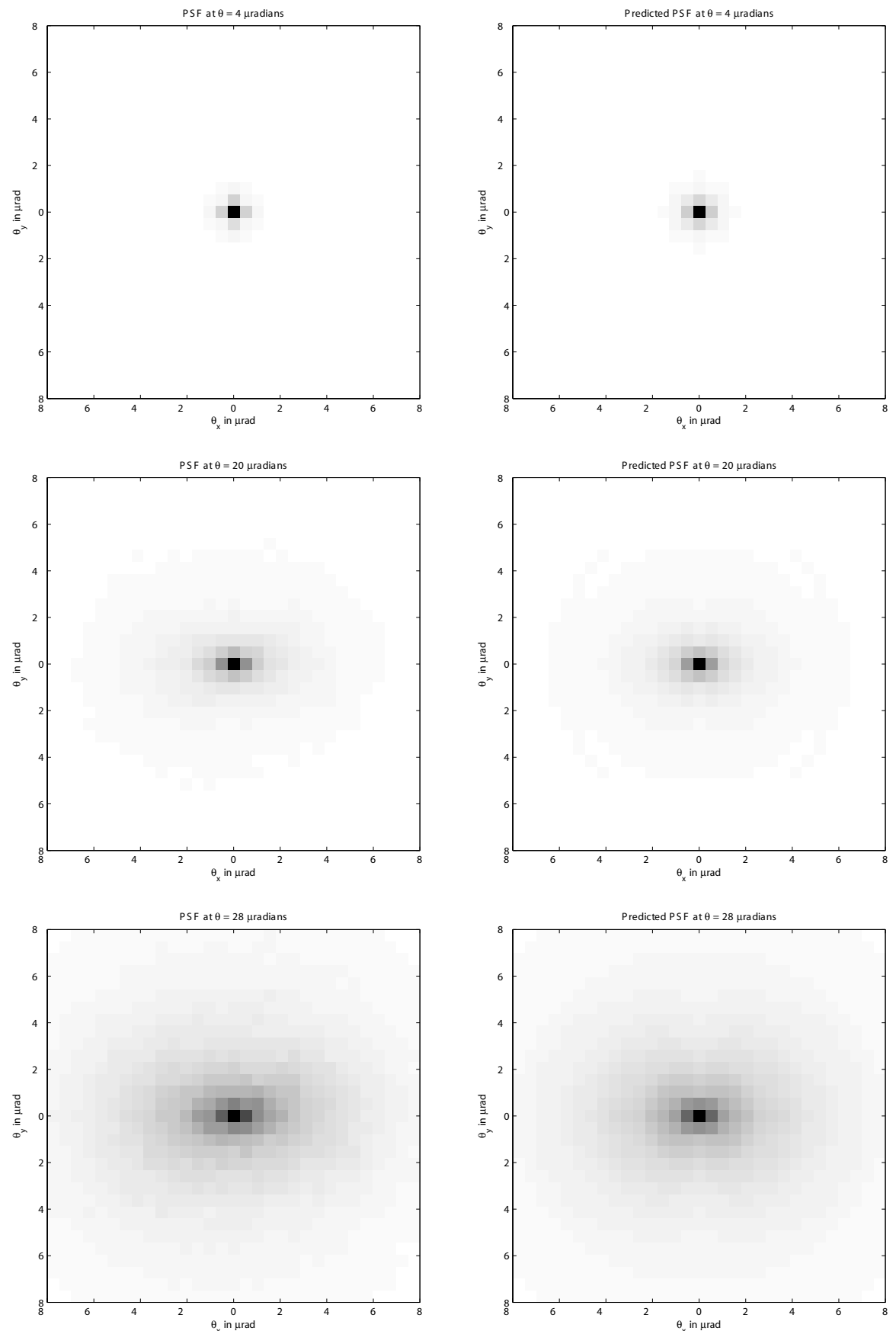

Figure 1.18: Predicted and simulated PSF's for $\theta=4$, 20, and 28 pradians, and $r_{0}=$ $10 \mathrm{~cm}$. 


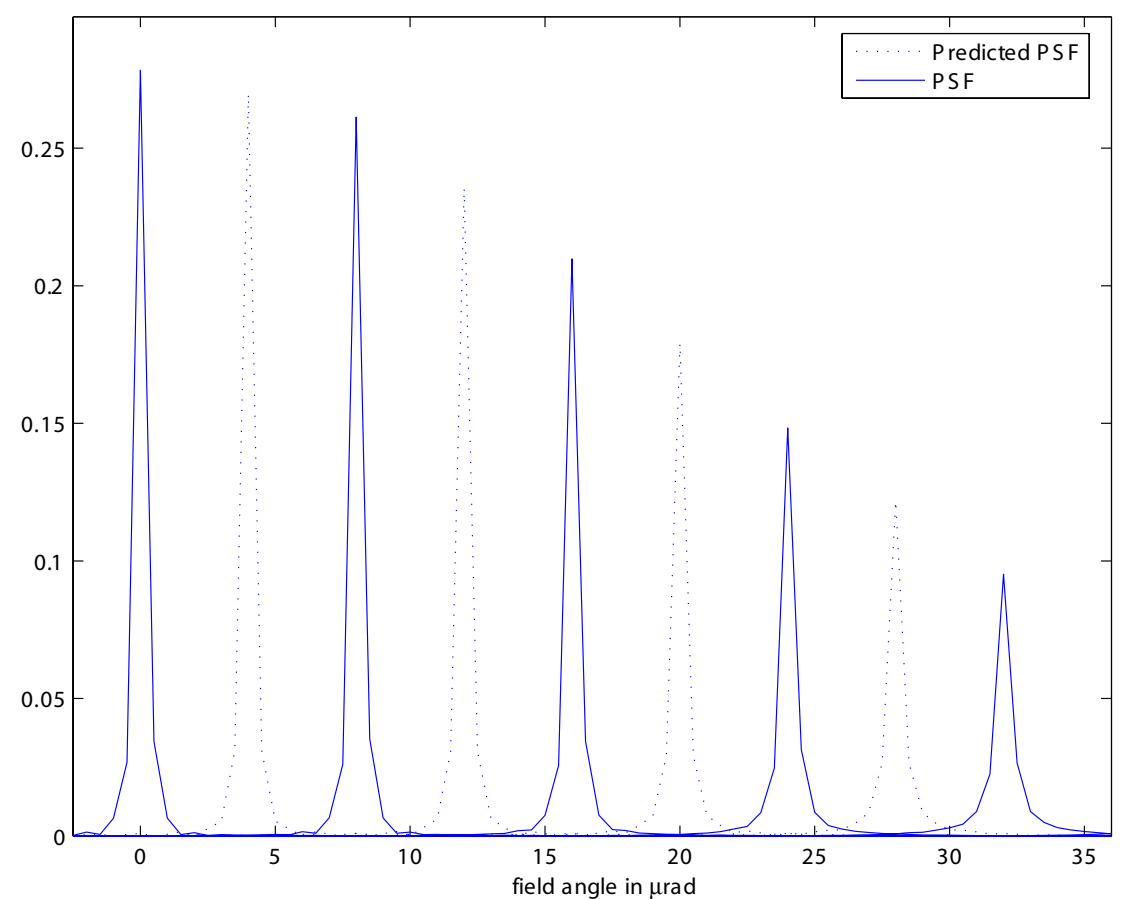

(a)

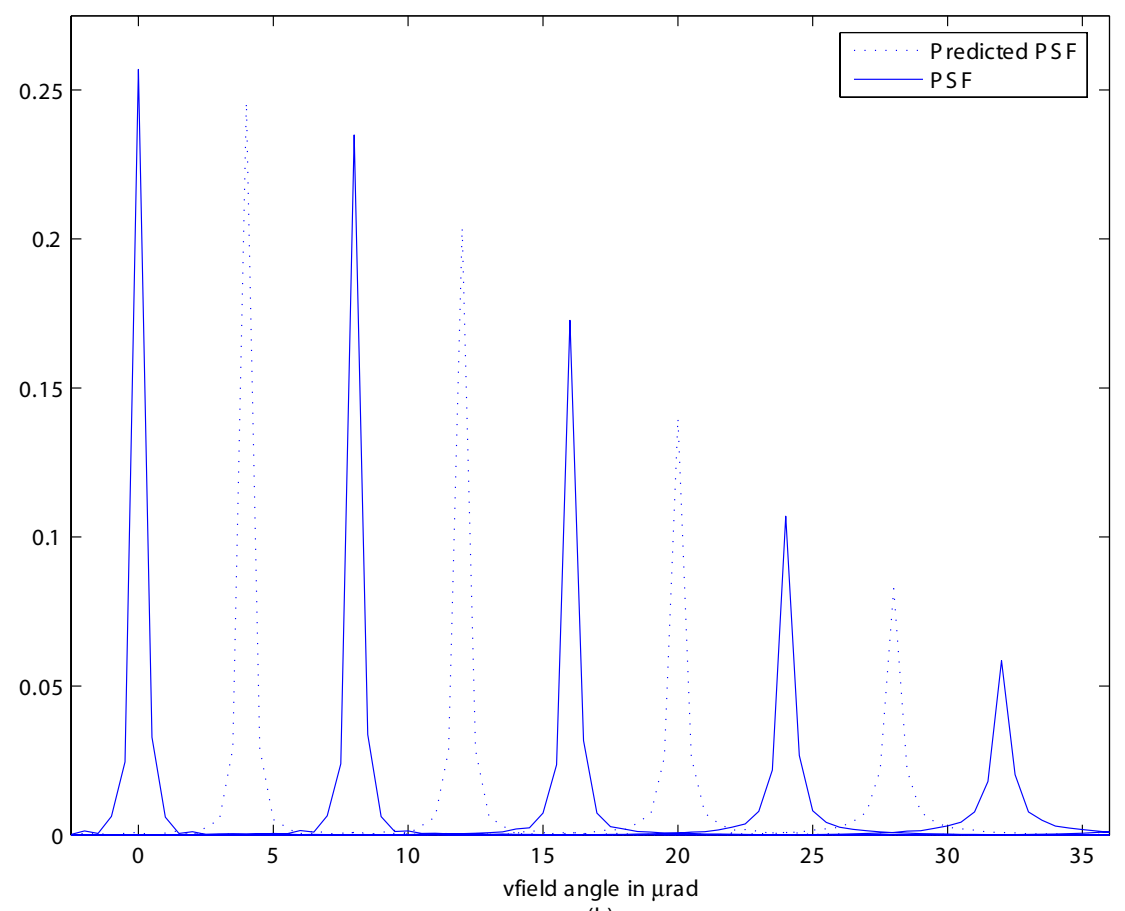

(b)

Figure 1.19: Cross-section of the simulated PSF at $\theta_{\text {known }}=0,8,16,24$ and $32 \mu \mathrm{rad}$ and the predicted PSF at $\theta_{\text {predict }}=4,12,20$ and $28 \mu \mathrm{rad}$, for (a) $r_{0}=25$ and $(b) r_{0}$ $=20 \mathrm{~cm}$. 


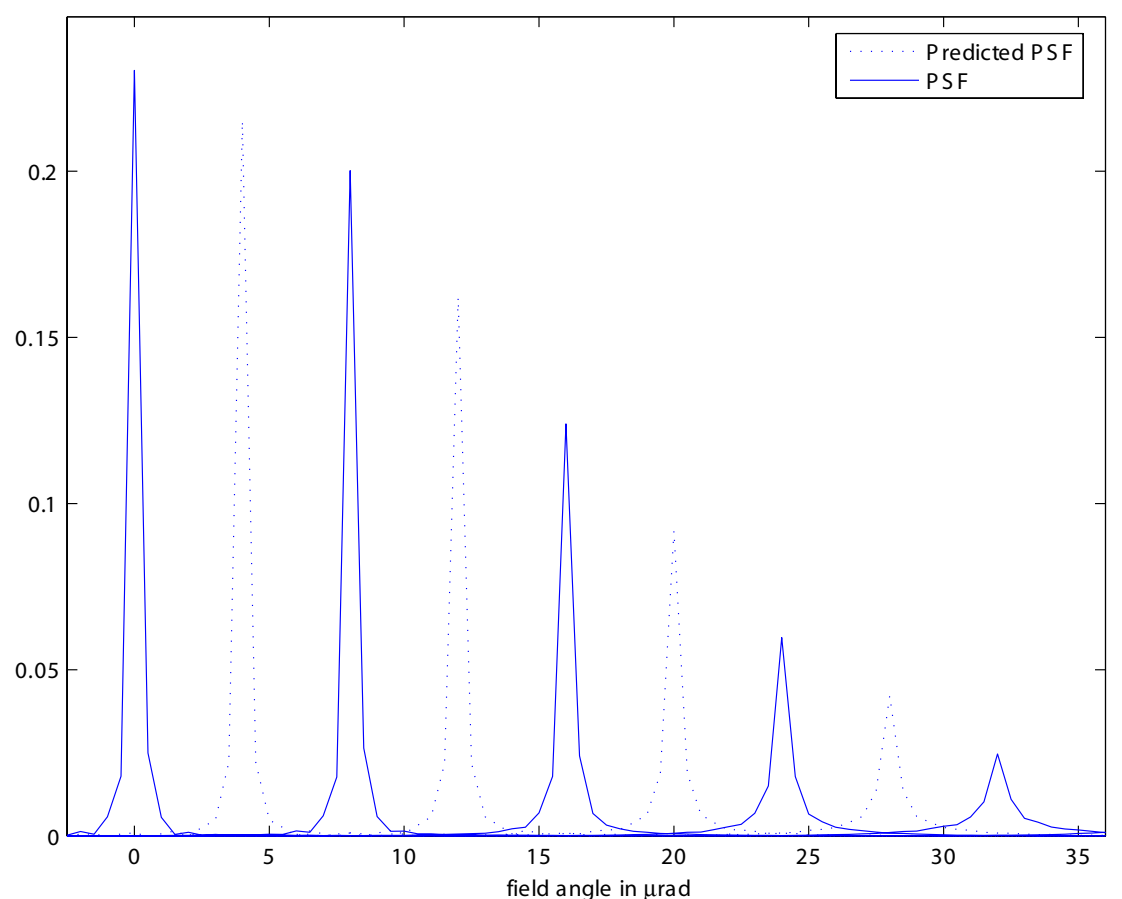

(a)

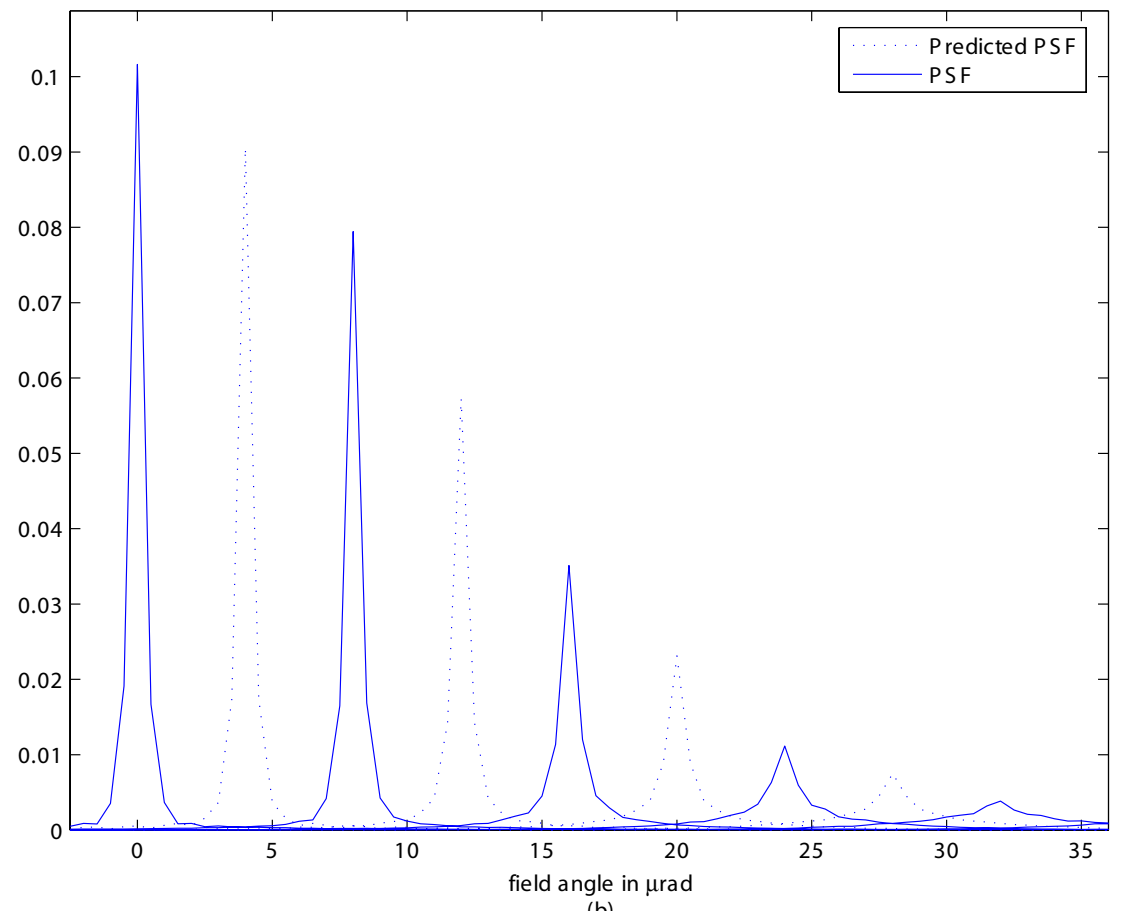

(b)

Figure 1.20: Cross-section of the simulated PSF at $\theta_{\text {known }}=0,8,16$, 24 and 32 $\mu$ rad and the predicted PSF at $\theta_{\text {predict }}=4,12,20$ and $28 \mu \mathrm{rad}$, for (a) $r_{0}=15$ and (b) $r_{0}$ $=10 \mathrm{~cm}$. 


\section{APPENDIX B}

Results: Reconstruction of anisoplanatic adaptive optics images 

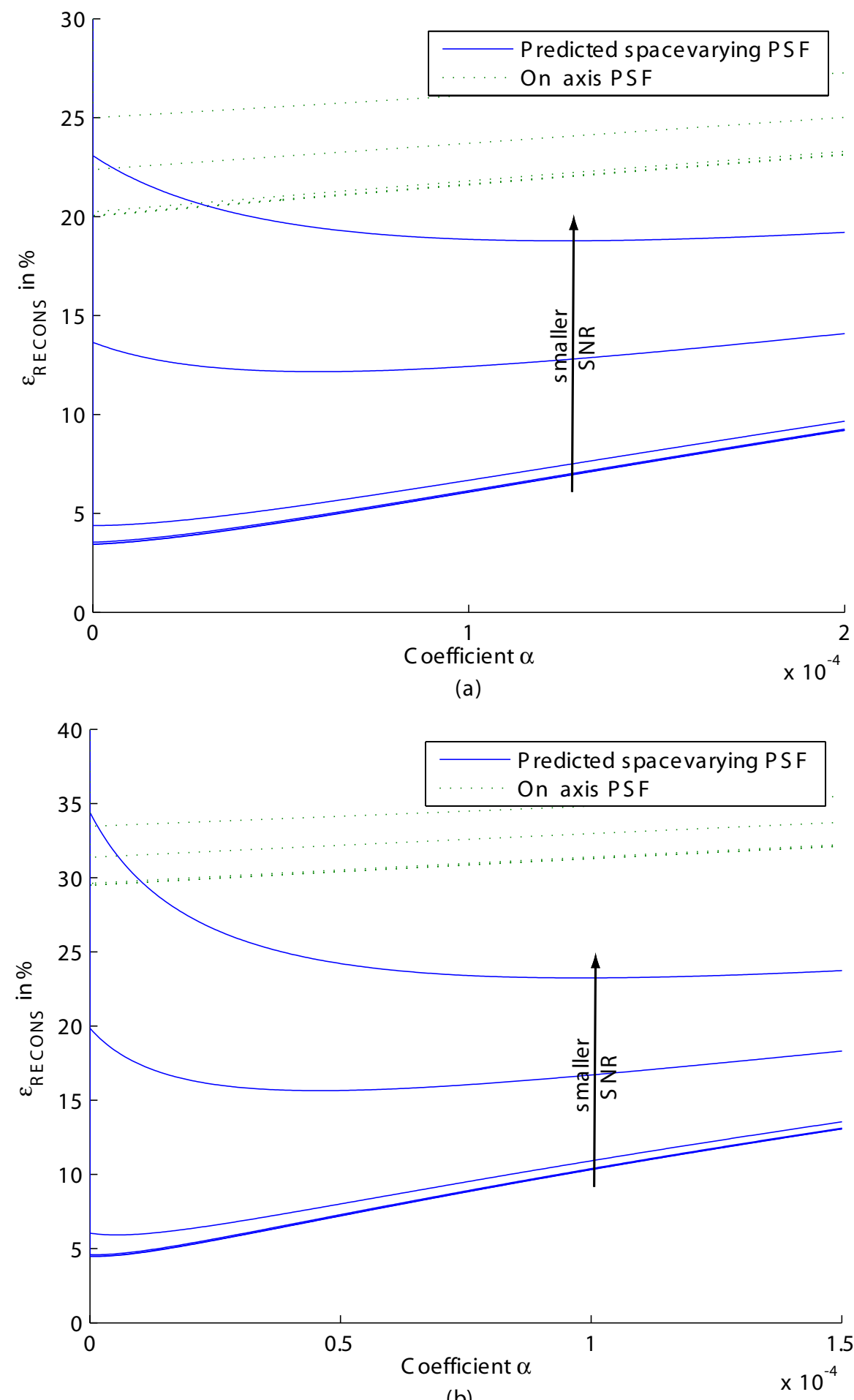

Figure 2.1: Reconstruction error $\epsilon_{R E C O N S}$ vs. coefficient $\alpha$ for (a) $r_{0}=25 \mathrm{~cm}$ and (b) $r_{0}=20 \mathrm{~cm}$. 


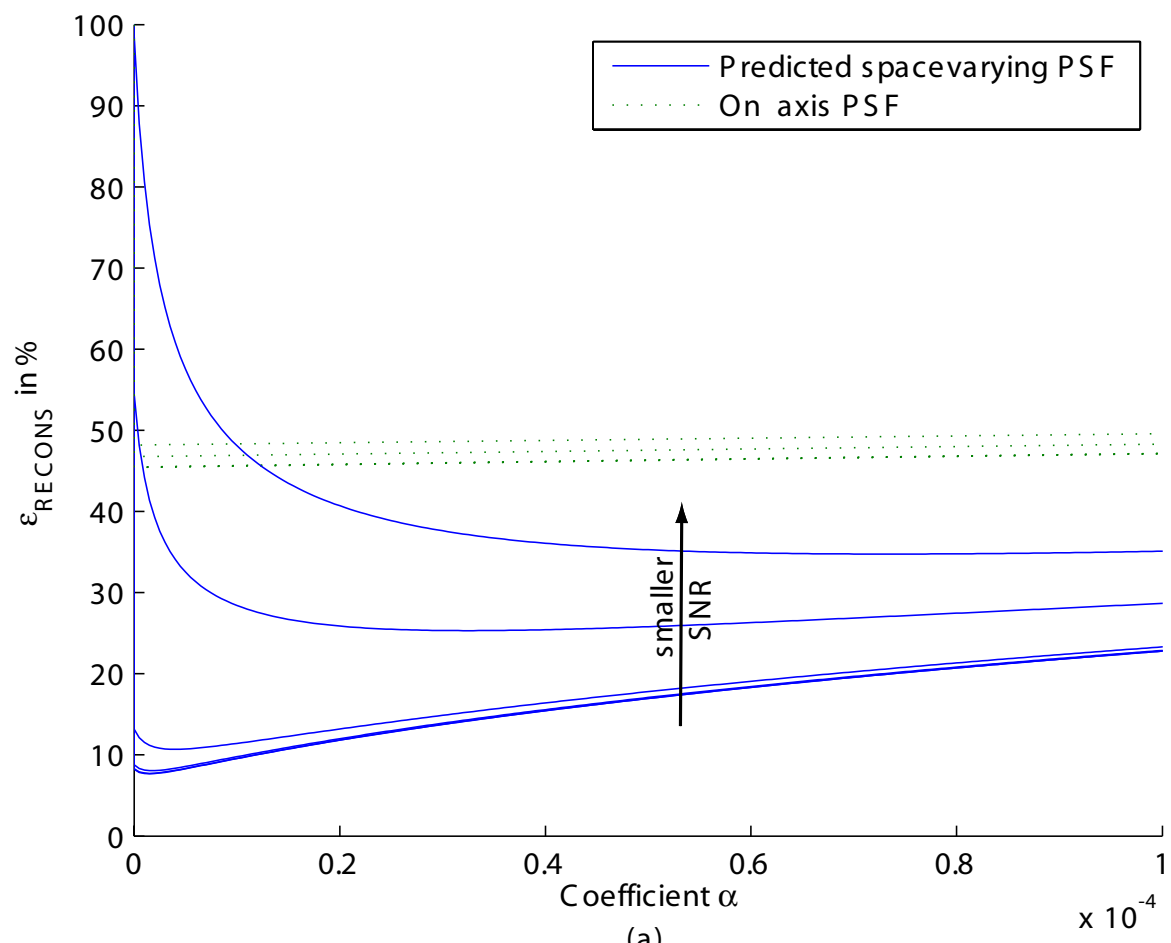

(a)

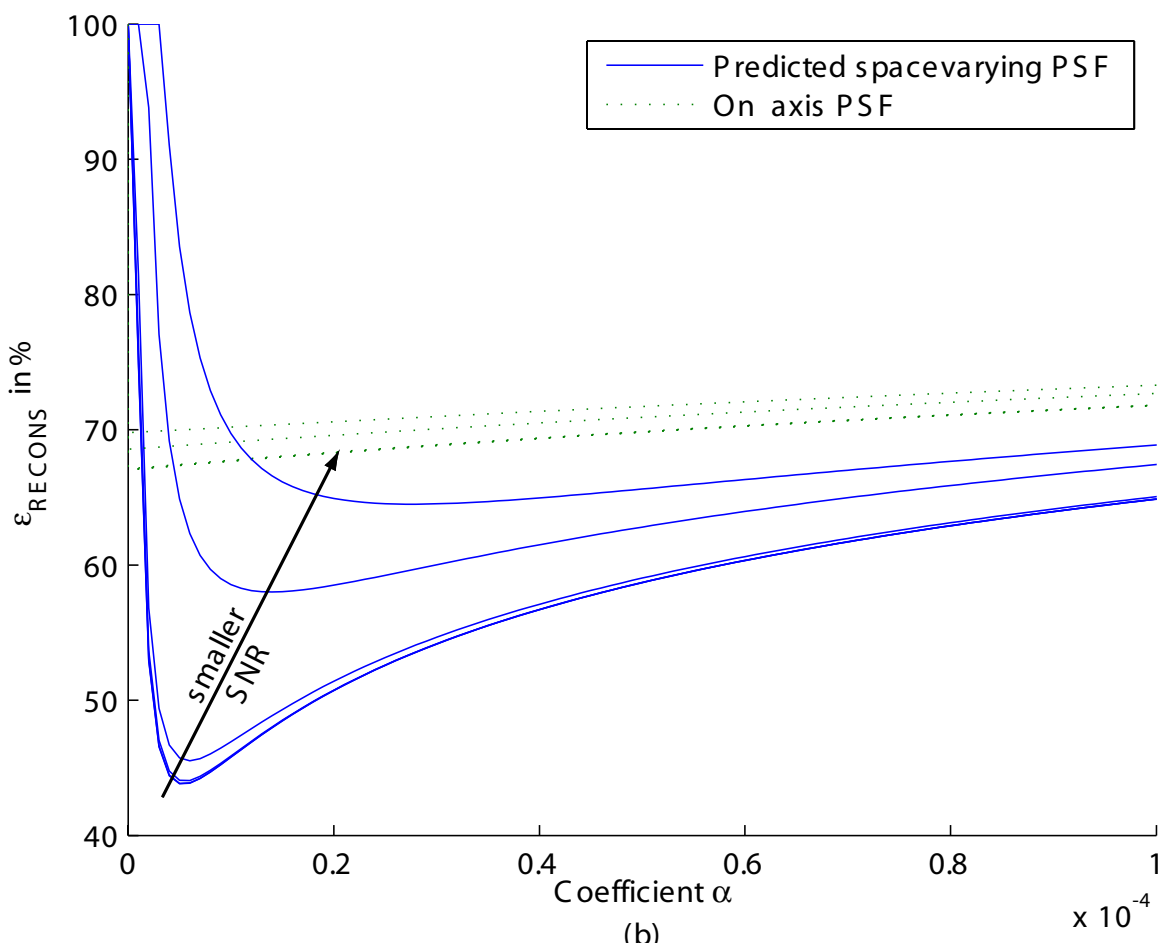

Figure 2.2: Reconstruction error $\epsilon_{R E C O N S}$ vs. coefficient $\alpha$ for (a) $r_{0}=15 \mathrm{~cm}$ and (b) $r_{0}=10 \mathrm{~cm}$. 


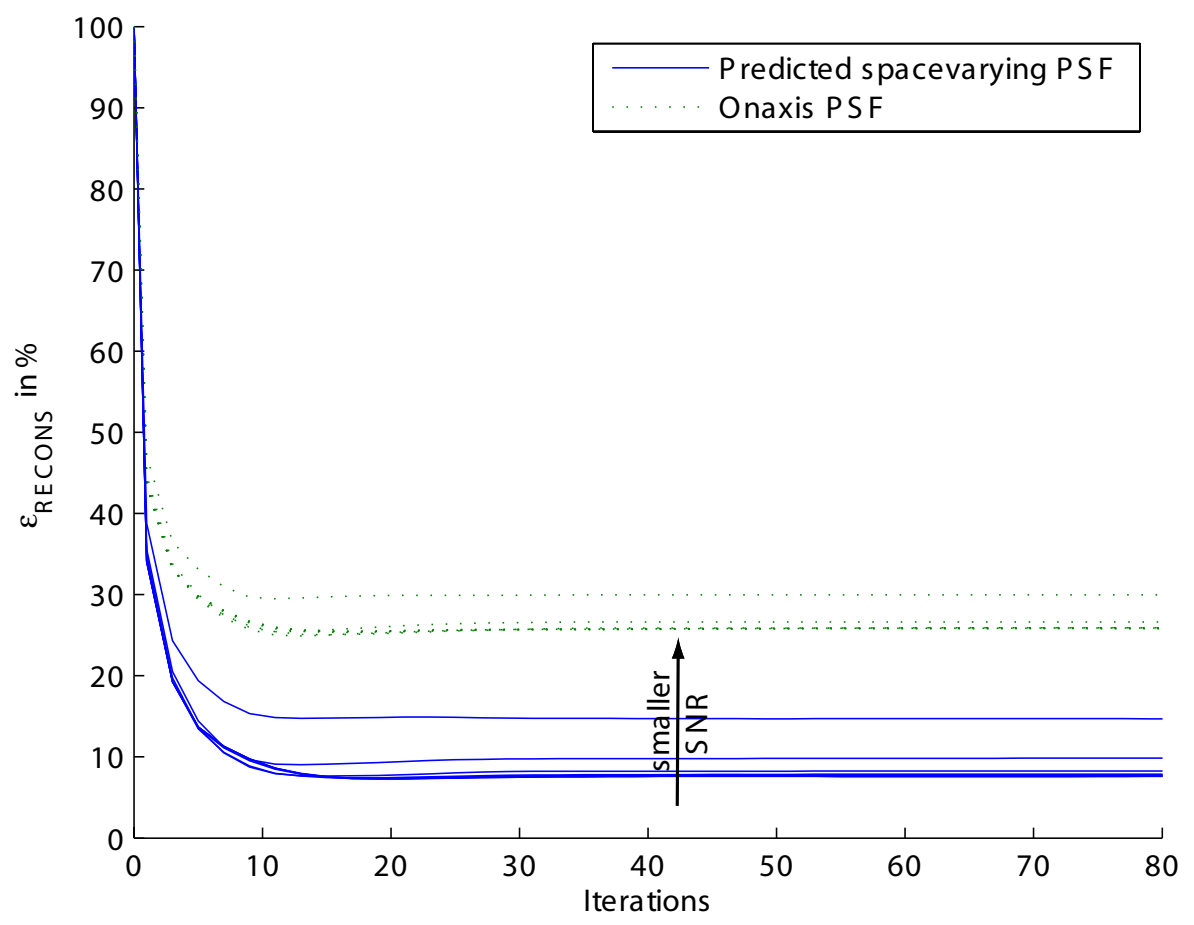

(a)

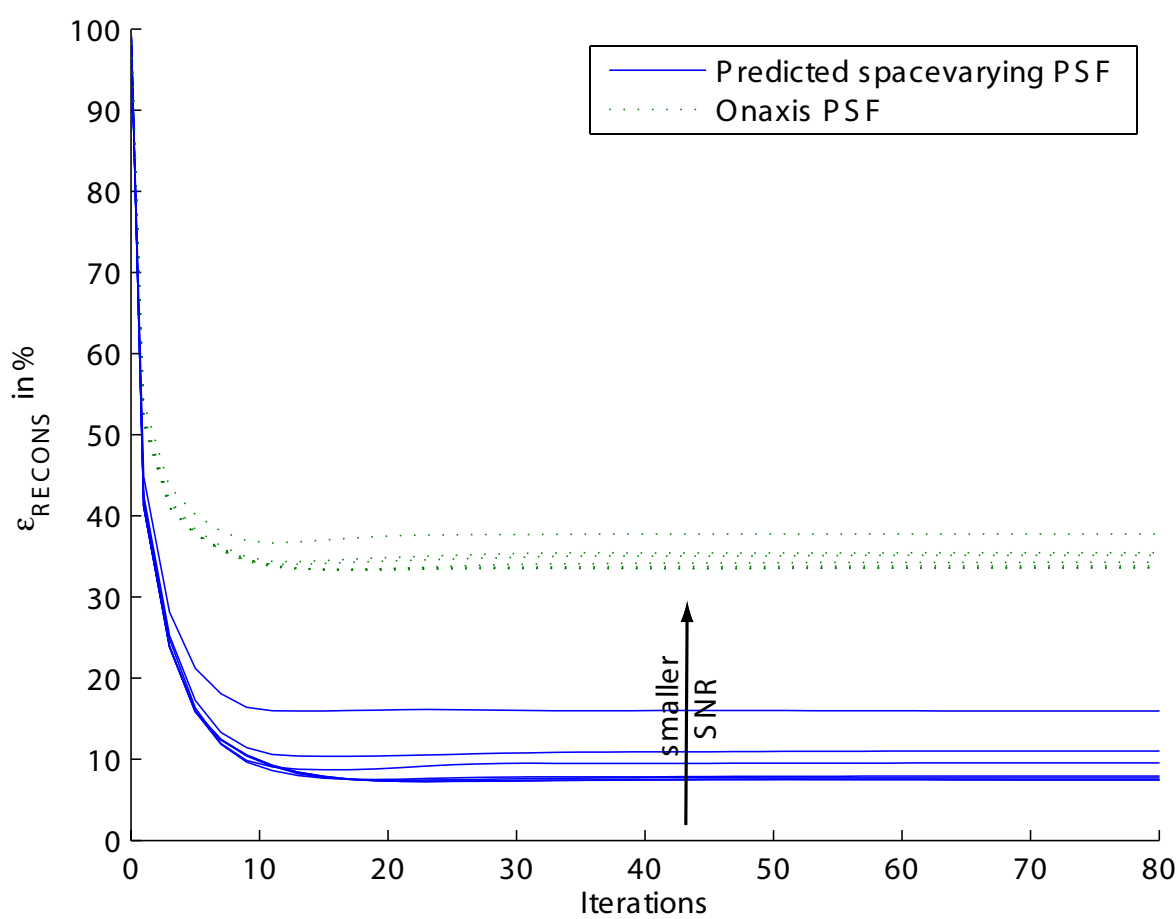

(b)

Figure 2.3: Reconstruction error $\epsilon_{\text {RECONS }}$ vs. iterations for (a) $r_{0}=25 \mathrm{~cm}$ and (b) $r_{0}=20 \mathrm{~cm}$. 


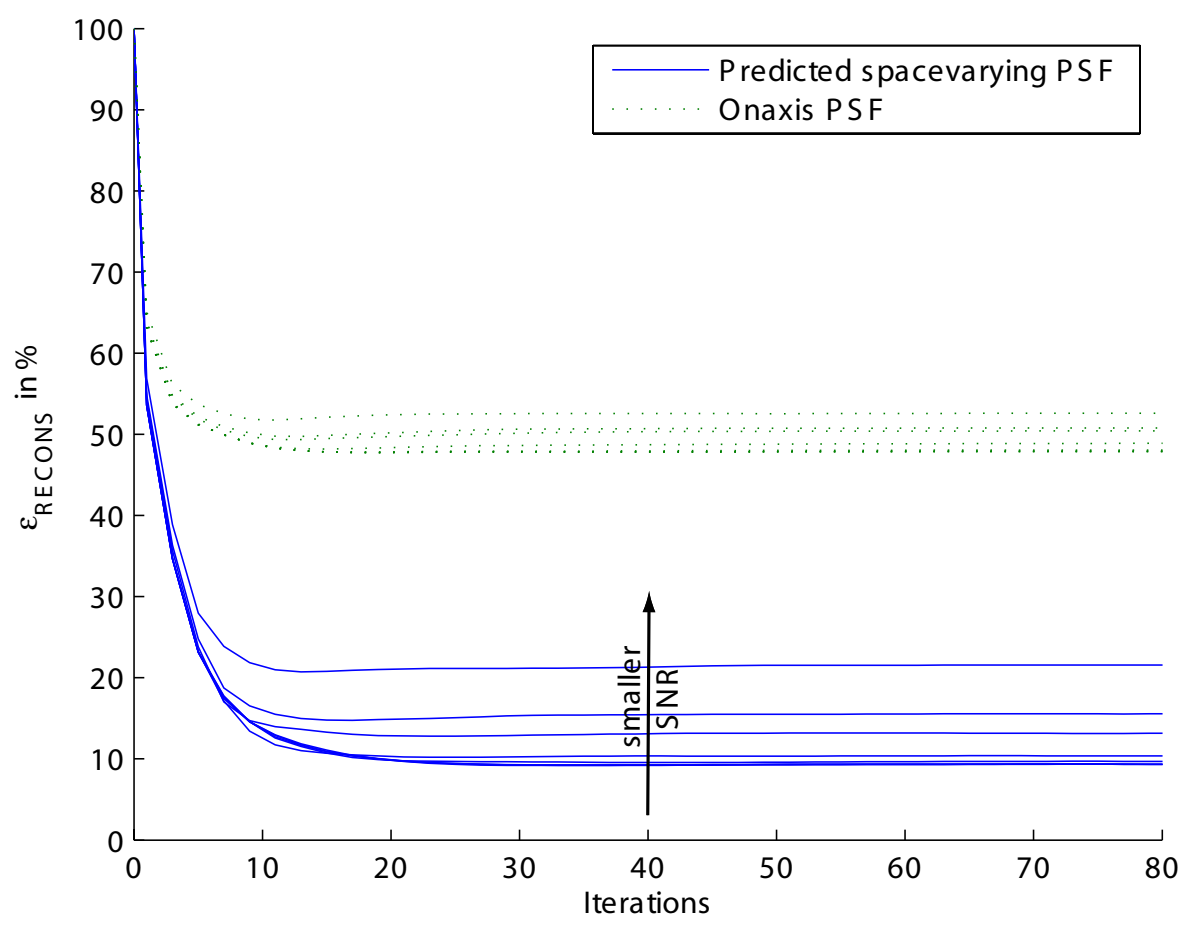

(a)

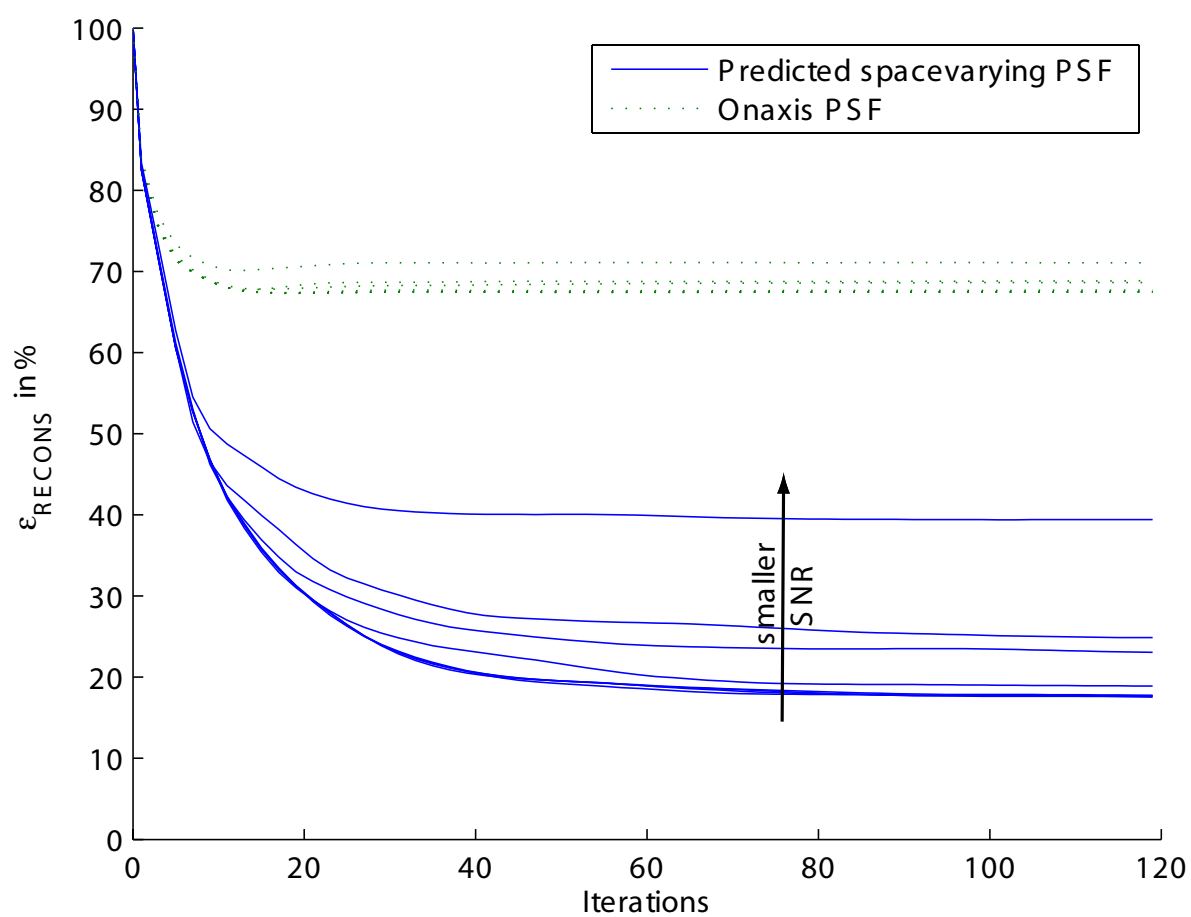

(b)

Figure 2.4: Reconstruction error $\epsilon_{R E C O N S}$ vs. iterations for (a) $r_{0}=15 \mathrm{~cm}$ and (b) $r_{0}=10 \mathrm{~cm}$. 


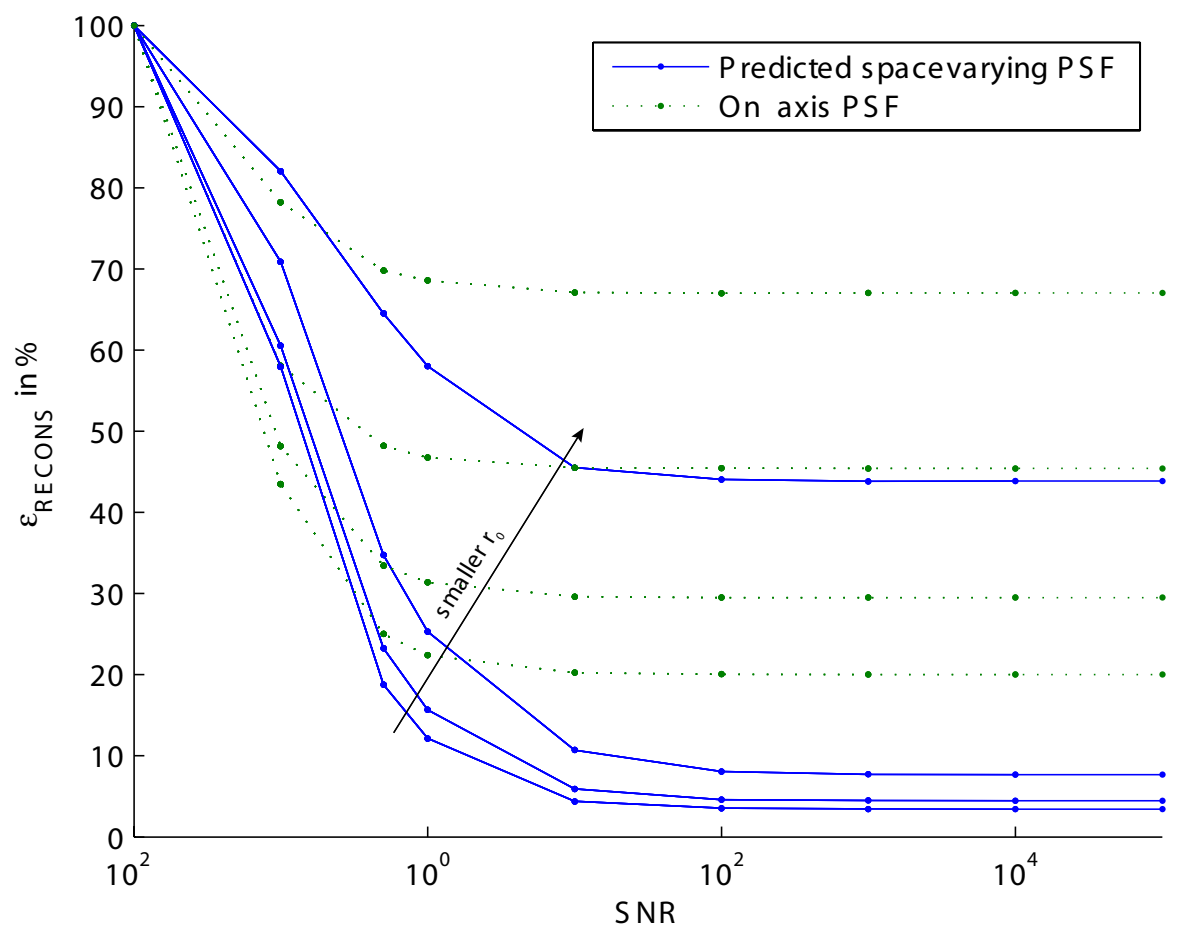

Figure 2.5: Reconstruction error $\epsilon_{R E C O N S}$ vs. SNR for the Tikhonov regularization and for different $r_{0}$. 


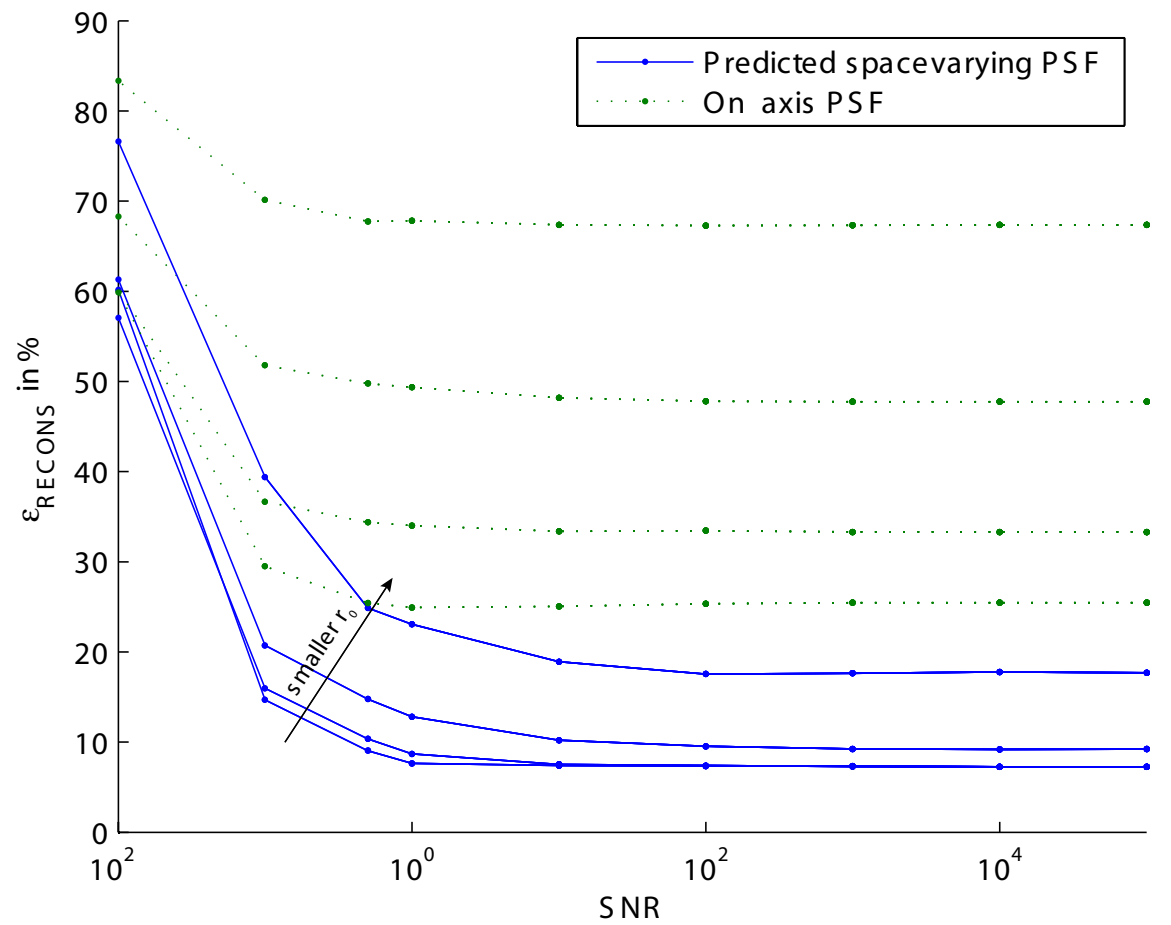

Figure 2.6: Reconstruction error $\epsilon_{R E C O N S}$ vs. SNR for the EM algorithm and for different $r_{0}$. 

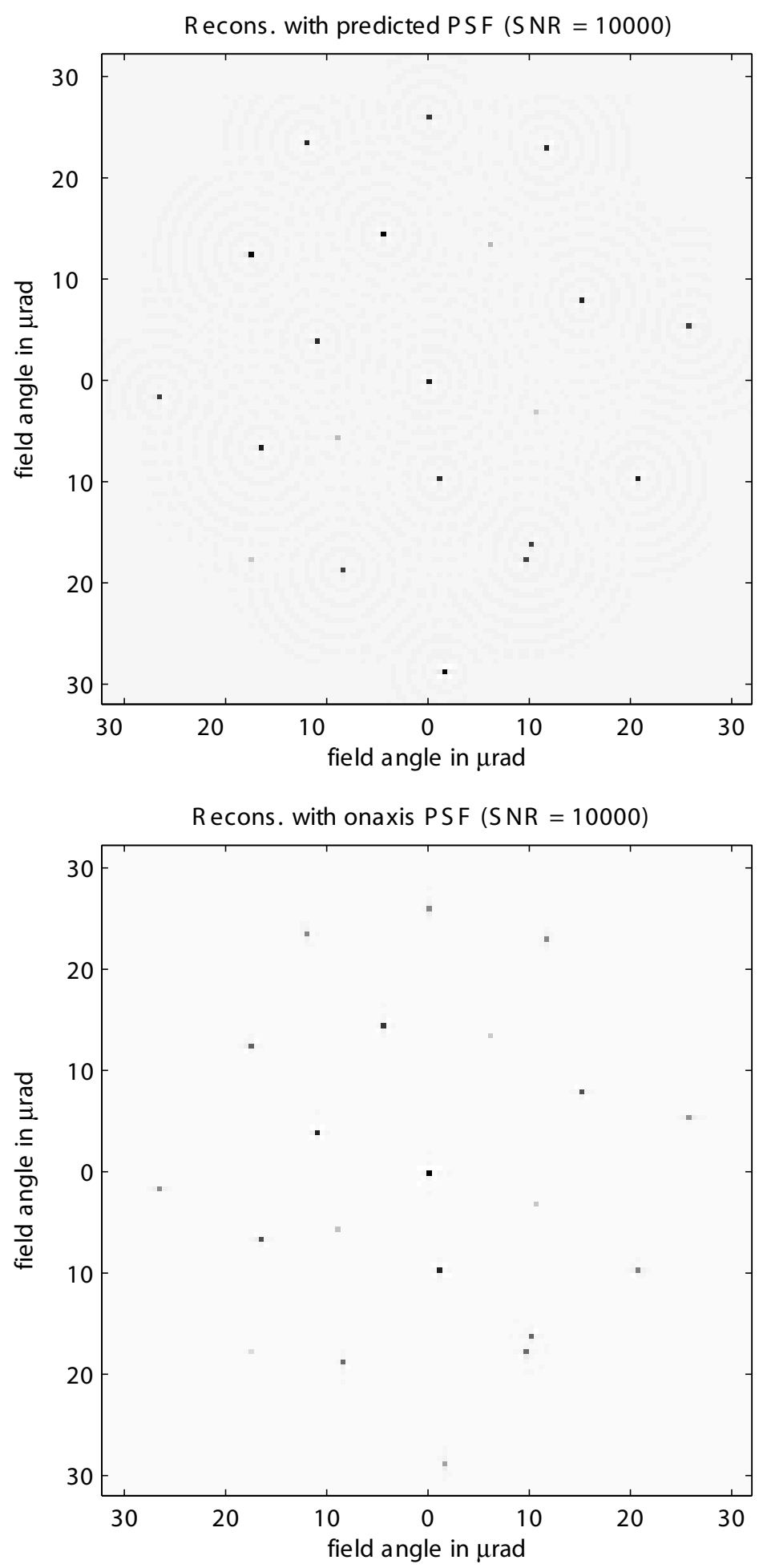

Figure 2.7: Reconstructed images using the Tikhonov regularization with the predicted $P S F$ and the on-axis PSF for $S N R=10000$ and $r_{0}=25 \mathrm{~cm}$. 

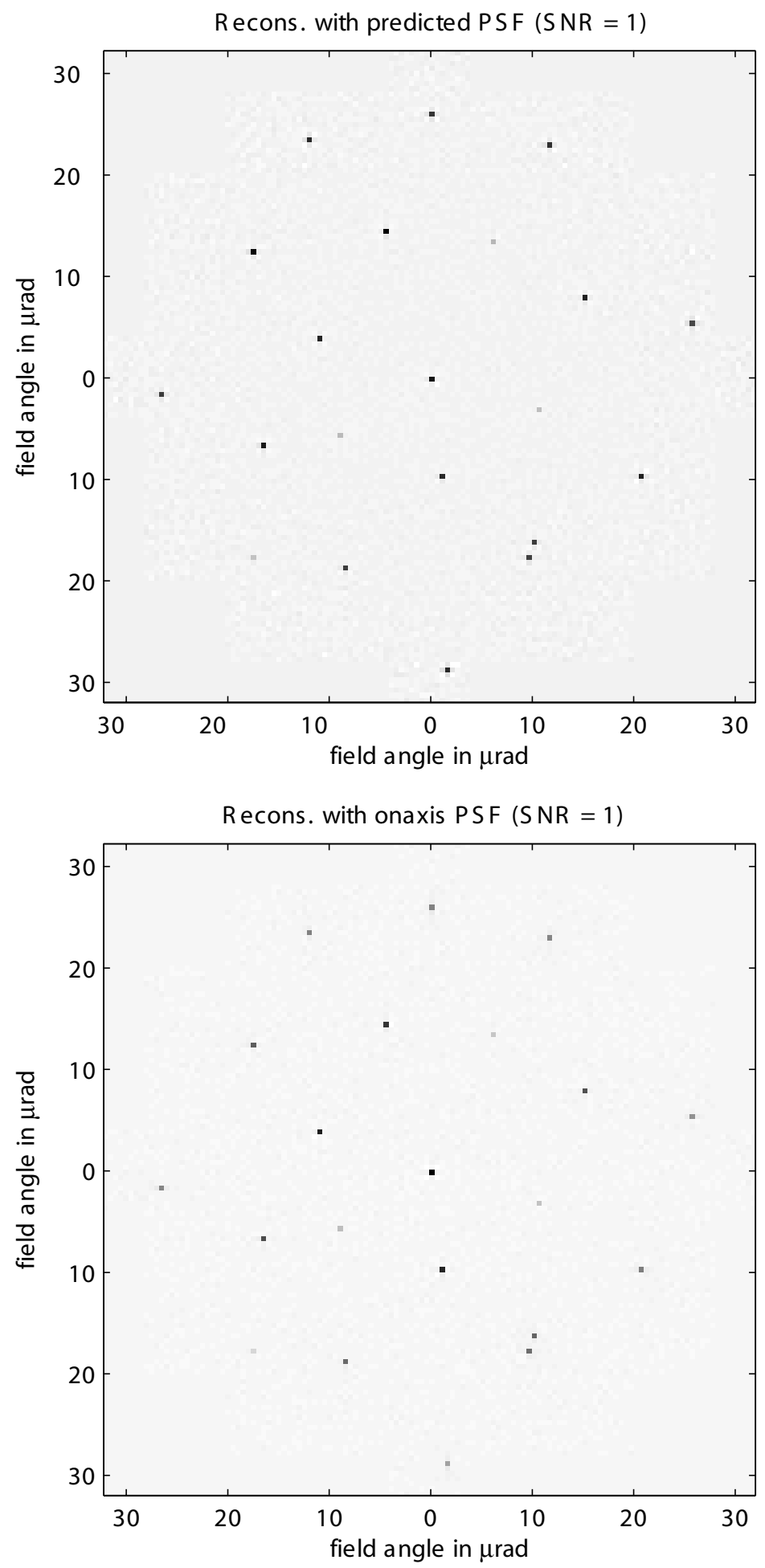

Figure 2.8: Reconstructed images using the Tikhonov regularization with the predicted $P S F$ and the on-axis PSF for $S N R=1$ and $r_{0}=25 \mathrm{~cm}$. 

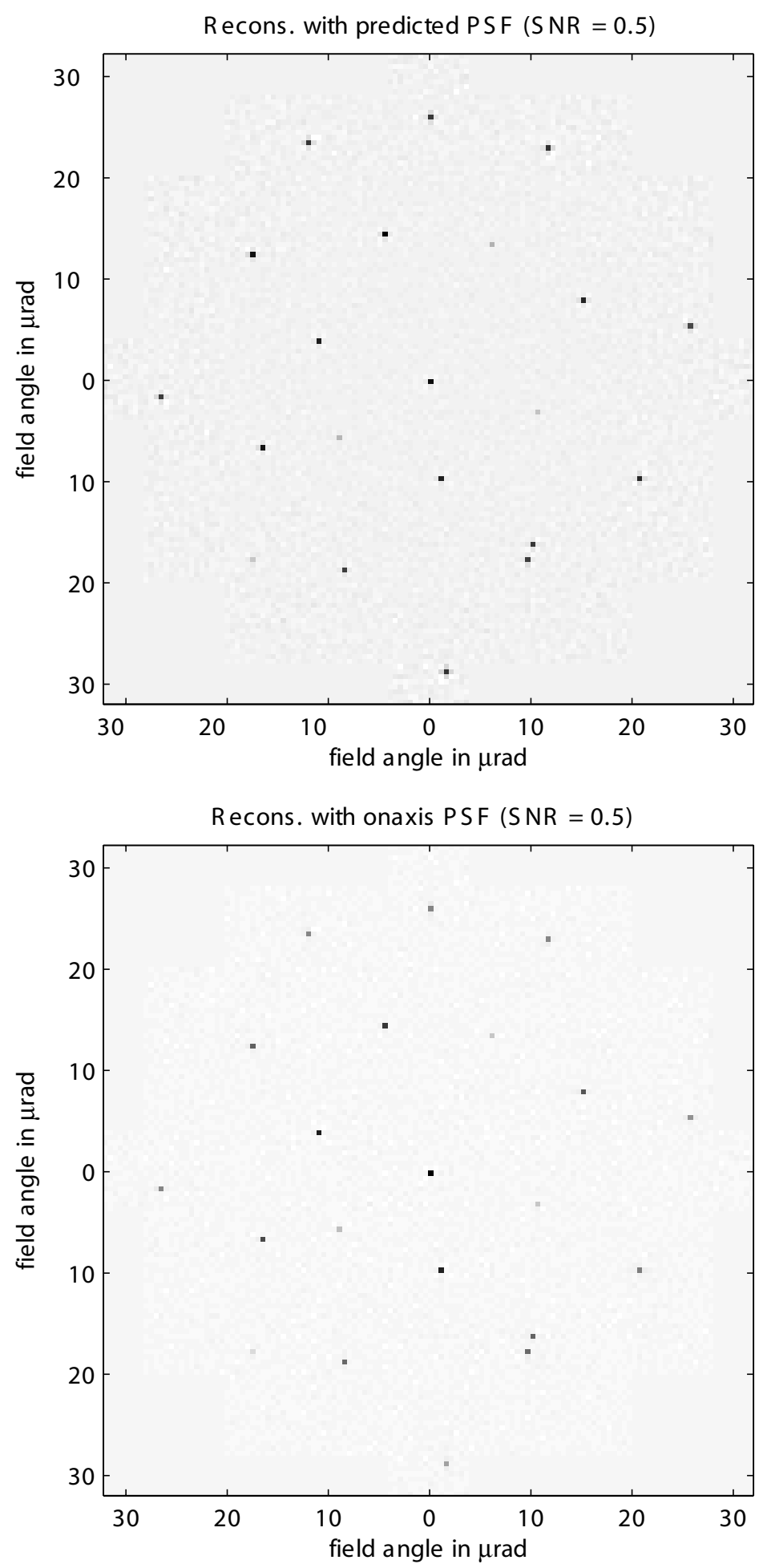

Figure 2.9: Reconstructed images using the Tikhonov regularization with the predicted $P S F$ and the on-axis PSF for $S N R=0.5$ and $r_{0}=25 \mathrm{~cm}$. 

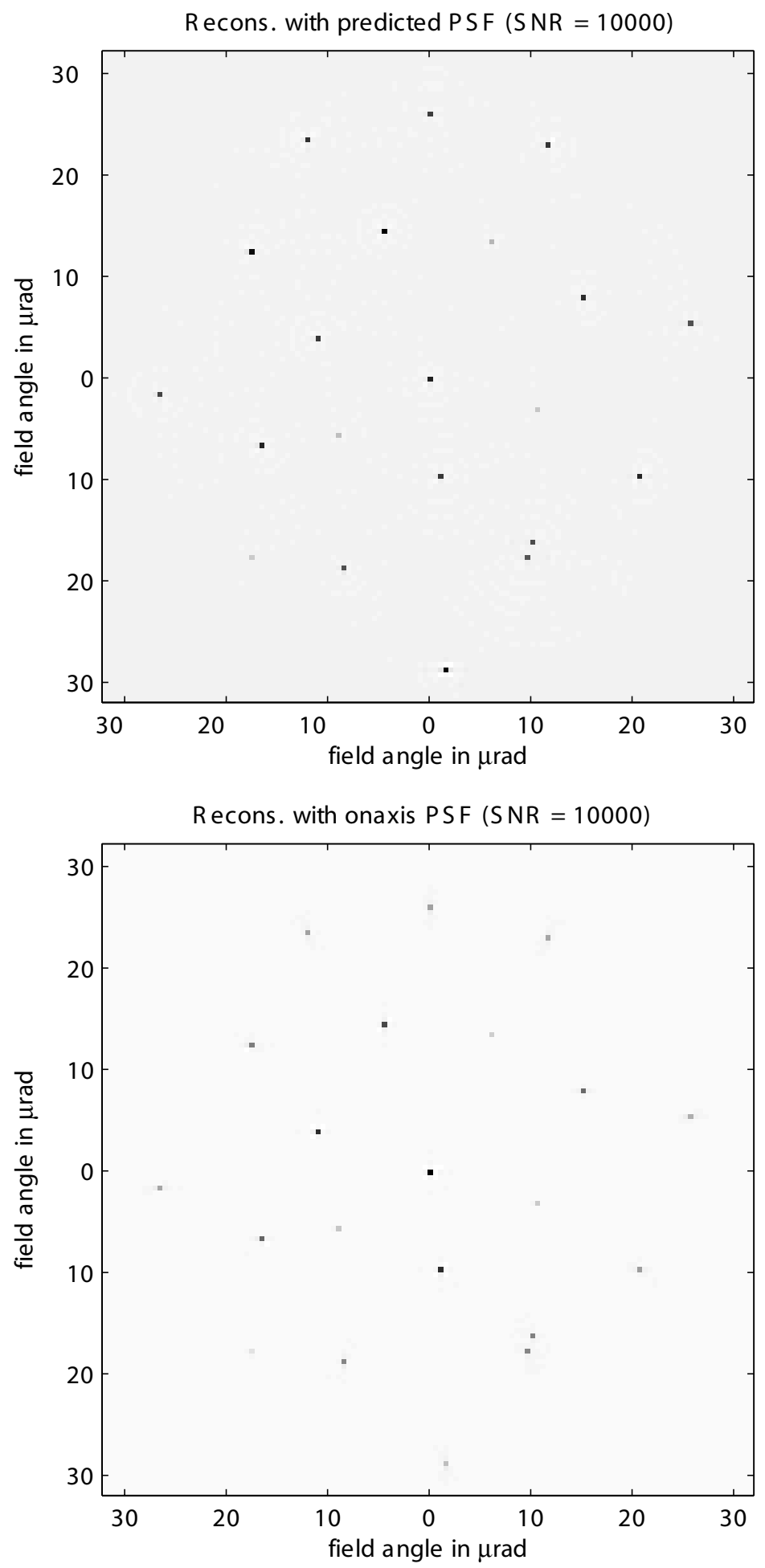

Figure 2.10: Reconstructed images using the Tikhonov regularization with the predicted $P S F$ and the on-axis PSF for $S N R=10000$ and $r_{0}=20 \mathrm{~cm}$. 

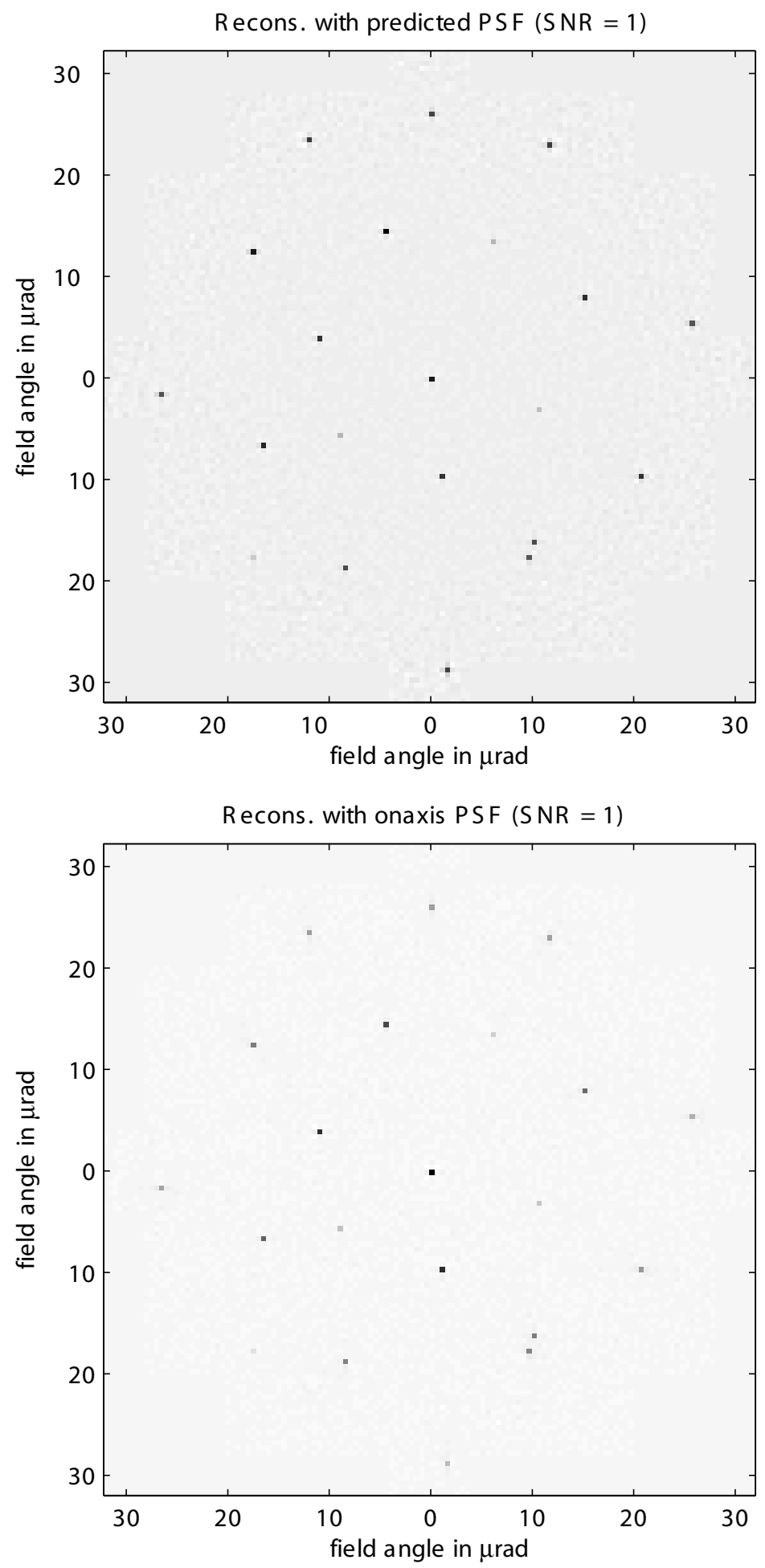

Figure 2.11: Reconstructed images using the Tikhonov regularization with the predicted $P S F$ and the on-axis PSF for $S N R=1$ and $r_{0}=20 \mathrm{~cm}$. 

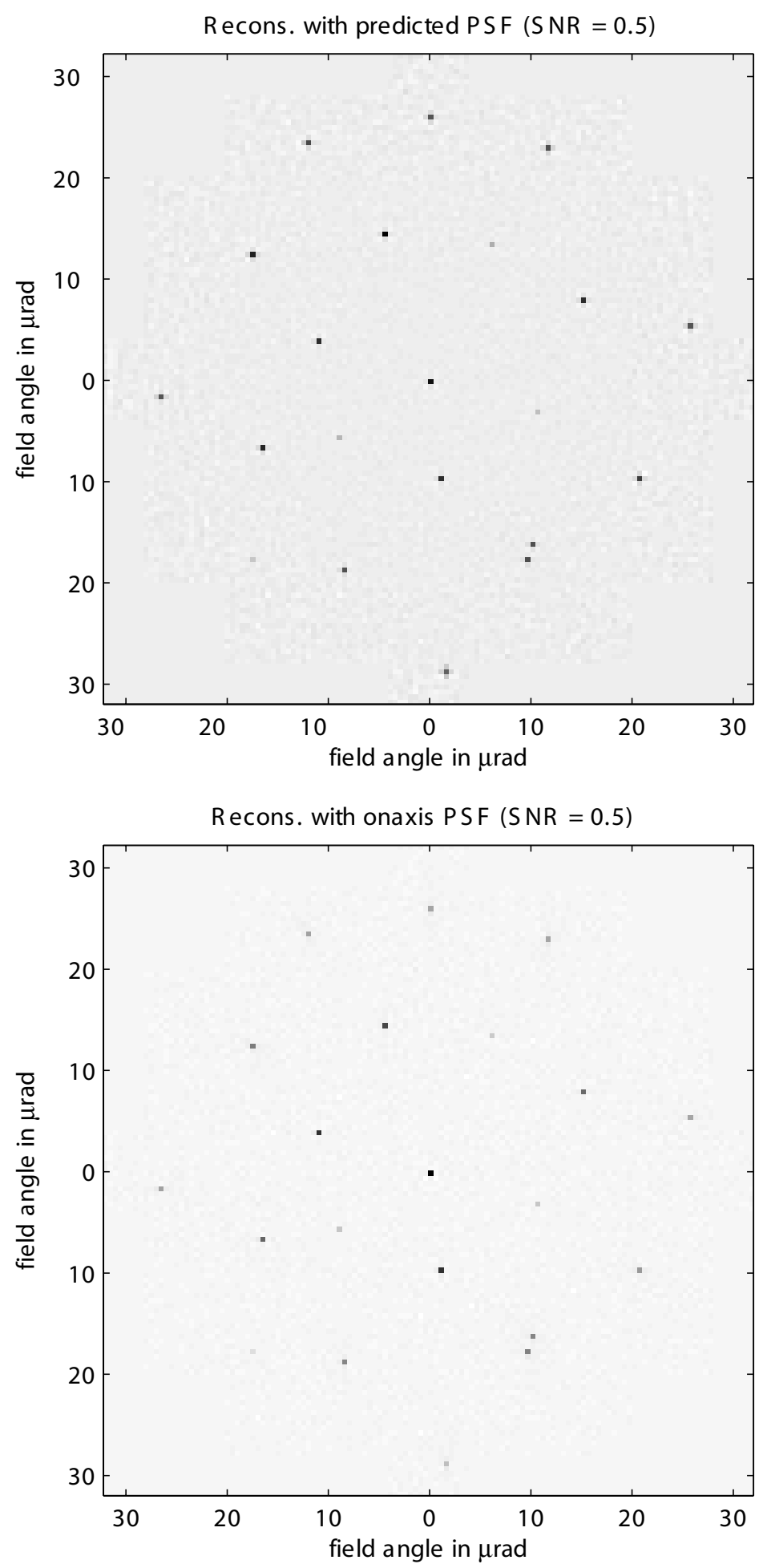

Figure 2.12: Reconstructed images using the Tikhonov regularization with the predicted $P S F$ and the on-axis PSF for $S N R=0.5$ and $r_{0}=20 \mathrm{~cm}$. 

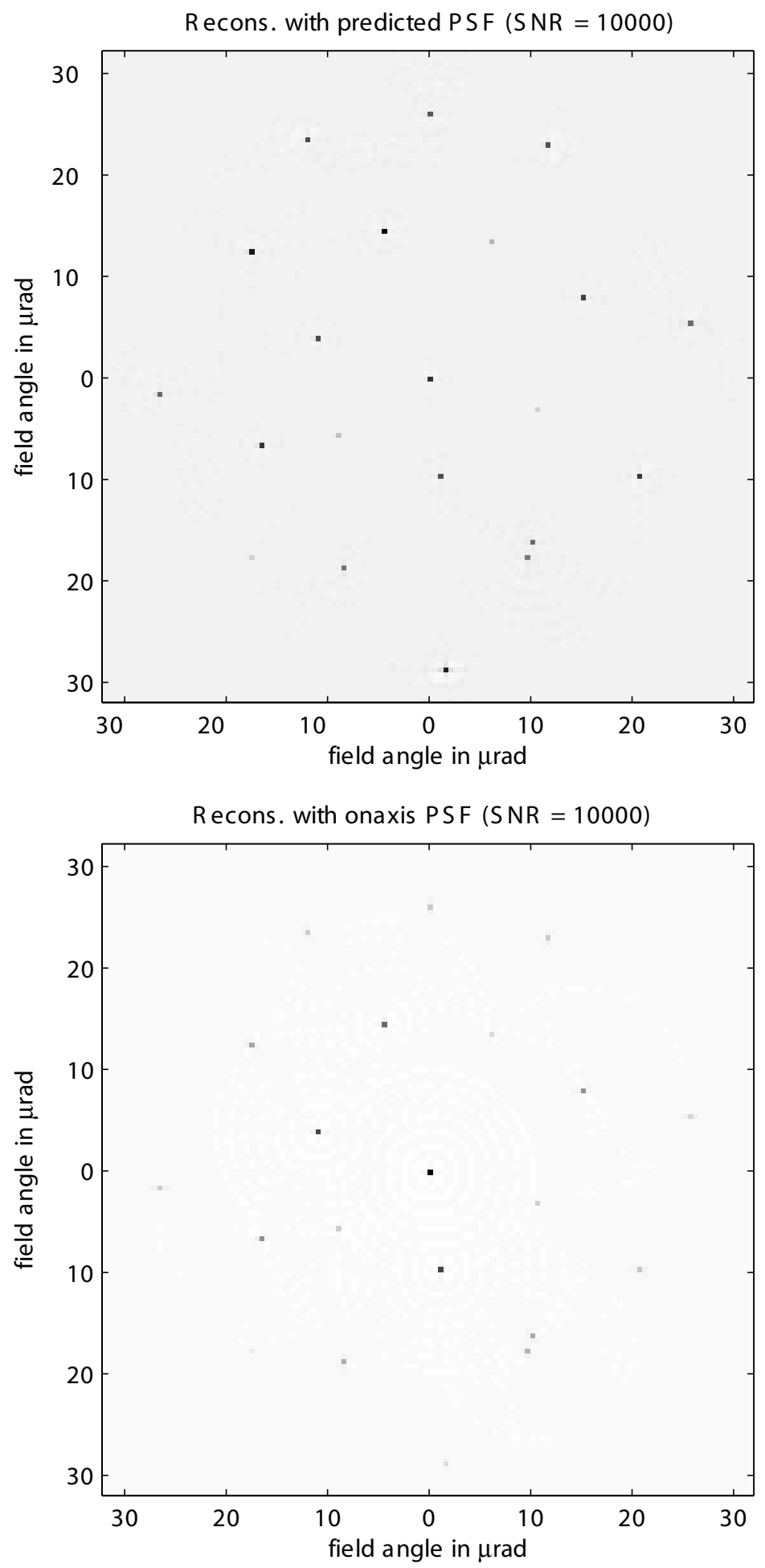

Figure 2.13: Reconstructed images using the Tikhonov regularization with the predicted $P S F$ and the on-axis PSF for $S N R=10000$ and $r_{0}=15 \mathrm{~cm}$. 

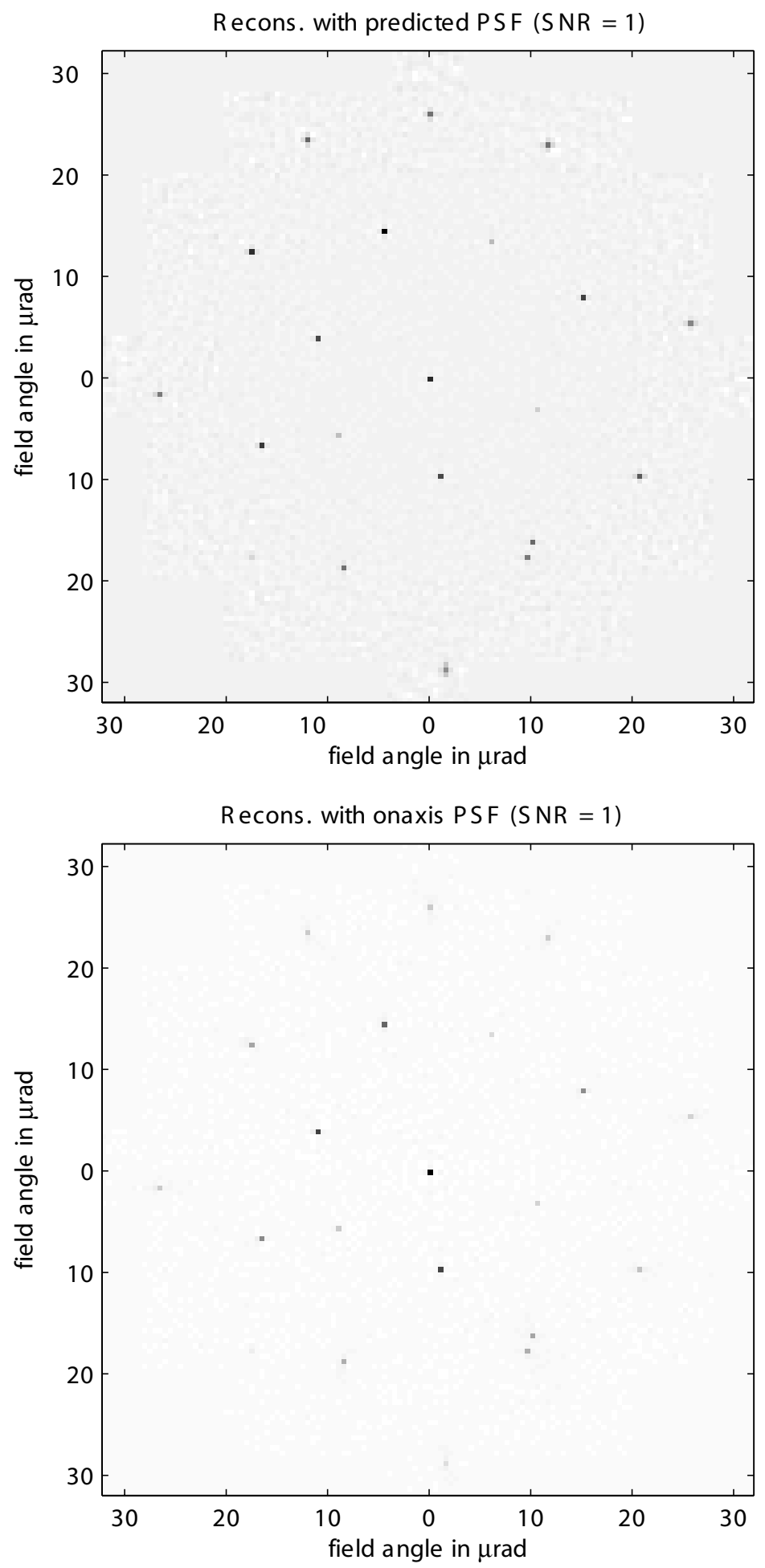

Figure 2.14: Reconstructed images using the Tikhonov regularization with the predicted $P S F$ and the on-axis PSF for $S N R=1$ and $r_{0}=15 \mathrm{~cm}$. 

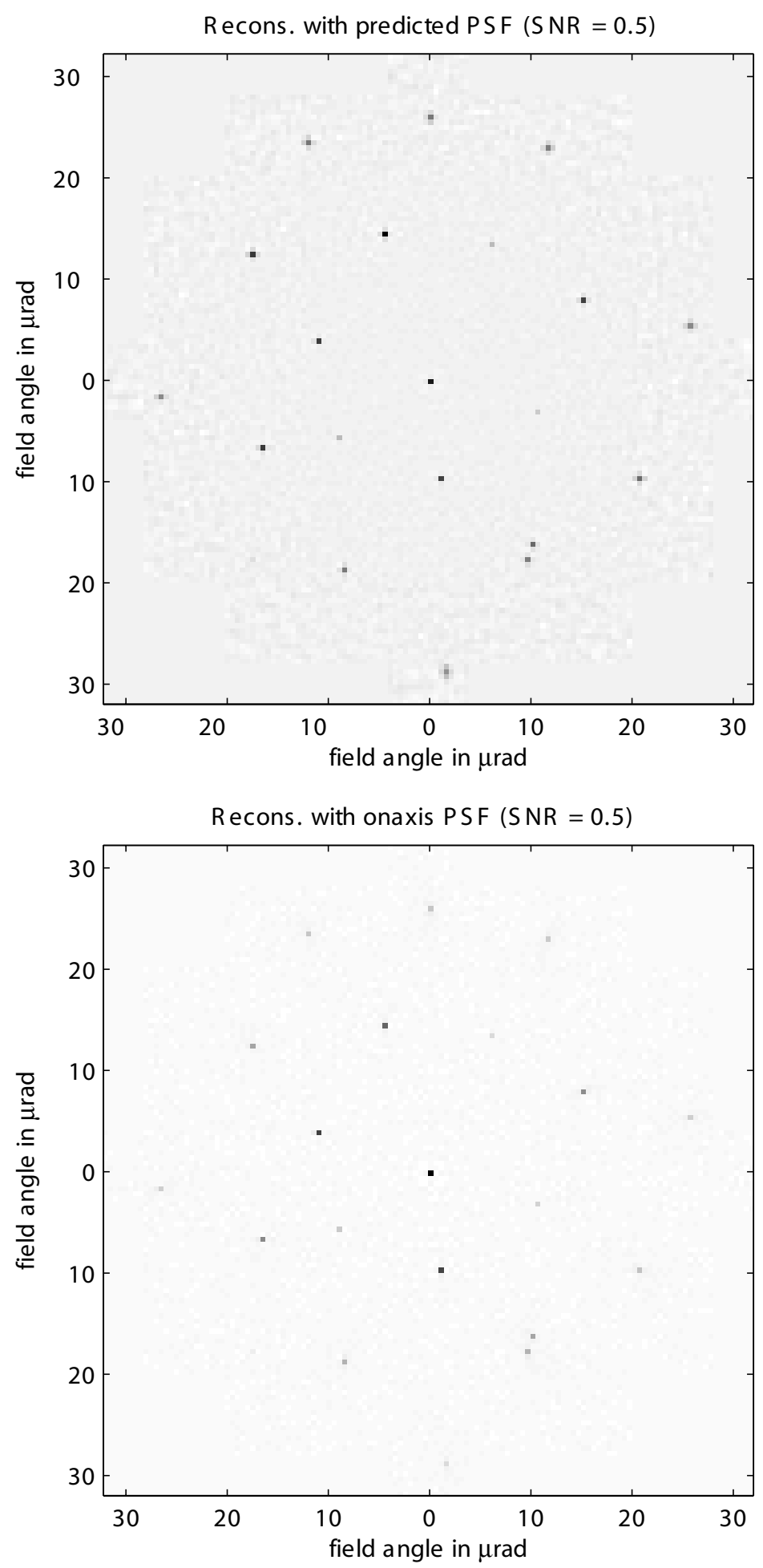

Figure 2.15: Reconstructed images using the Tikhonov regularization with the predicted $P S F$ and the on-axis PSF for $S N R=0.5$ and $r_{0}=15 \mathrm{~cm}$. 

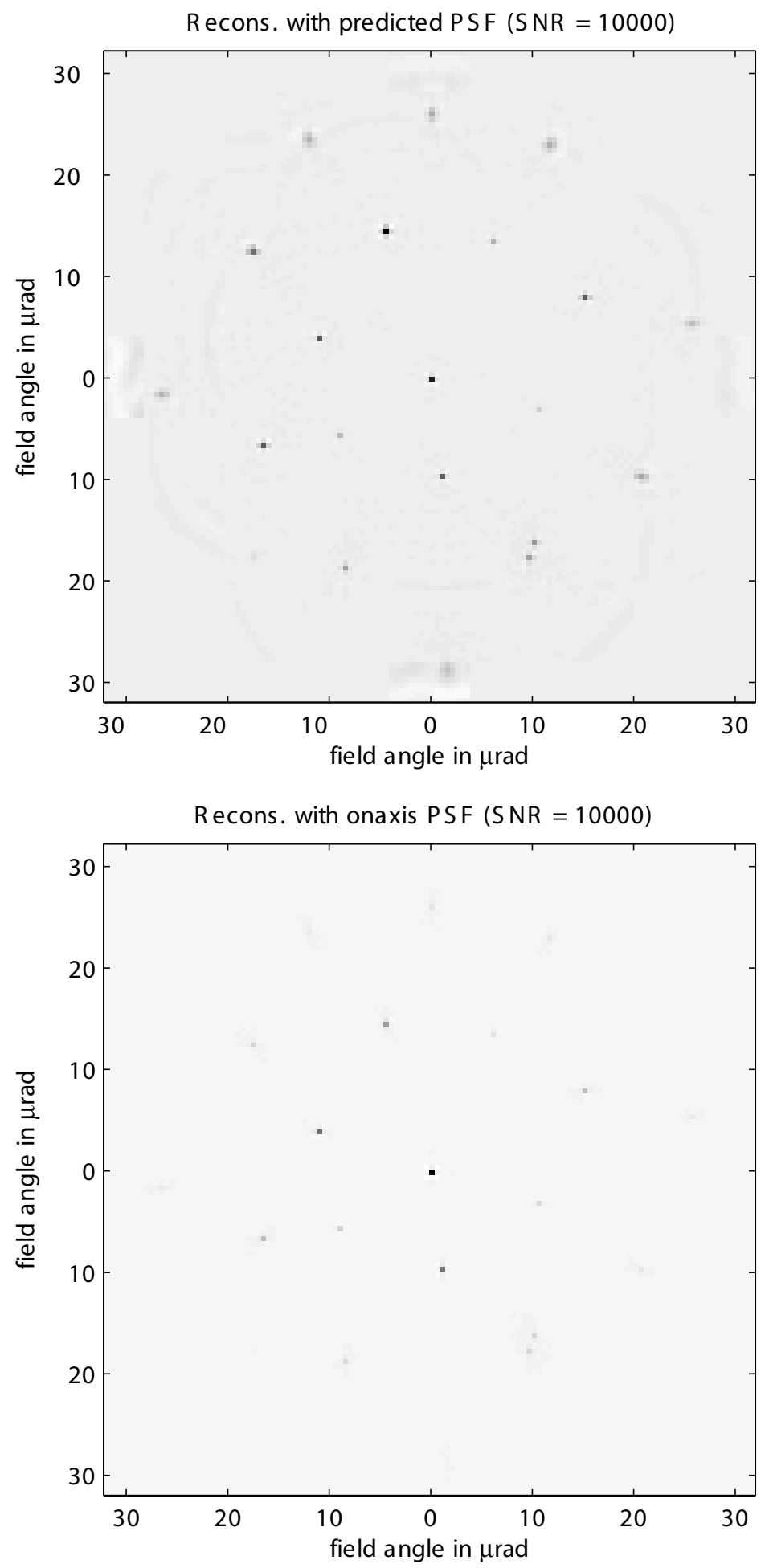

Figure 2.16: Reconstructed images using the Tikhonov regularization with the predicted $P S F$ and the on-axis PSF for $S N R=10000$ and $r_{0}=10 \mathrm{~cm}$. 

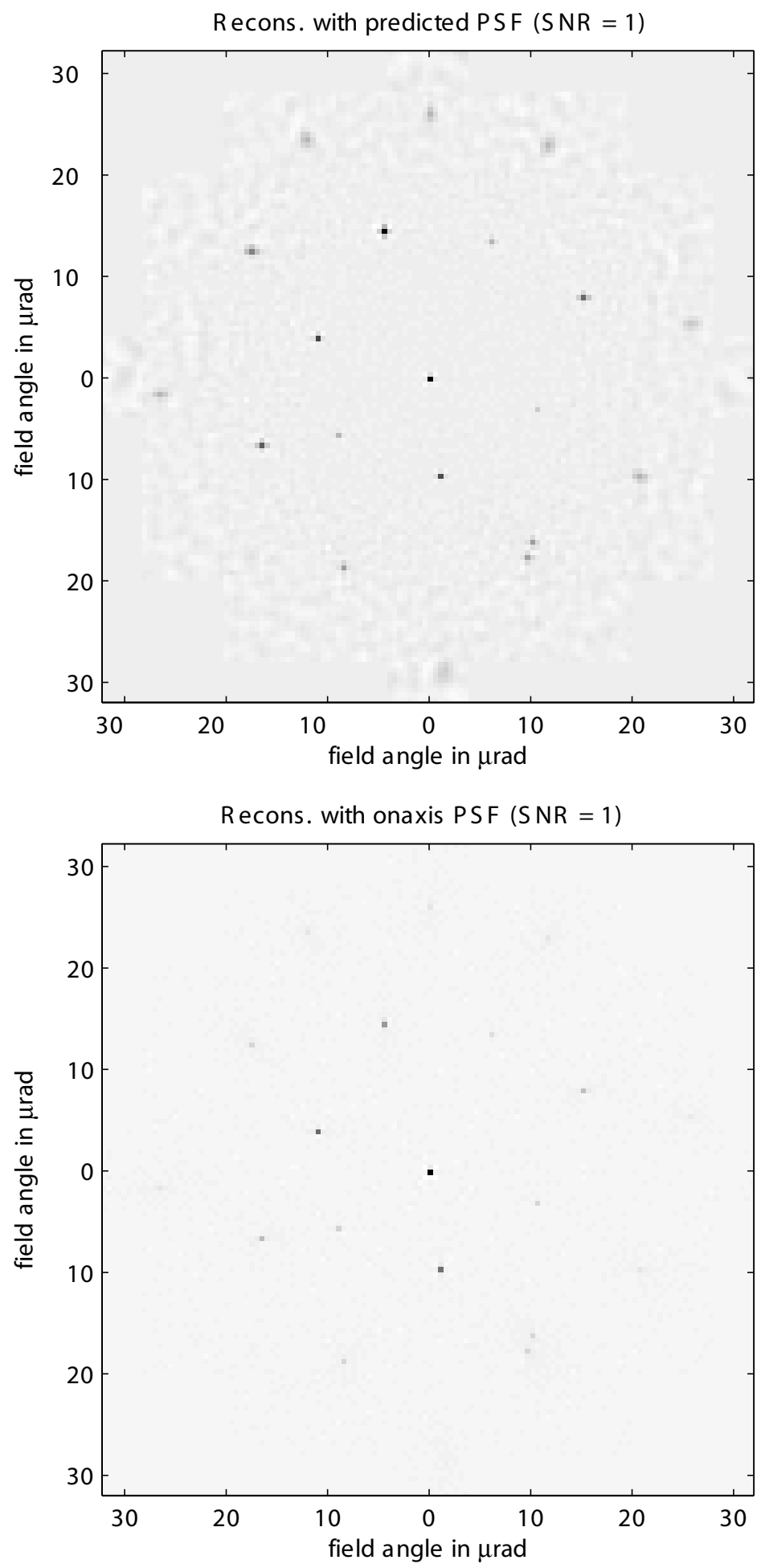

Figure 2.17: Reconstructed images using the Tikhonov regularization with the predicted $P S F$ and the on-axis PSF for $S N R=1$ and $r_{0}=10 \mathrm{~cm}$. 

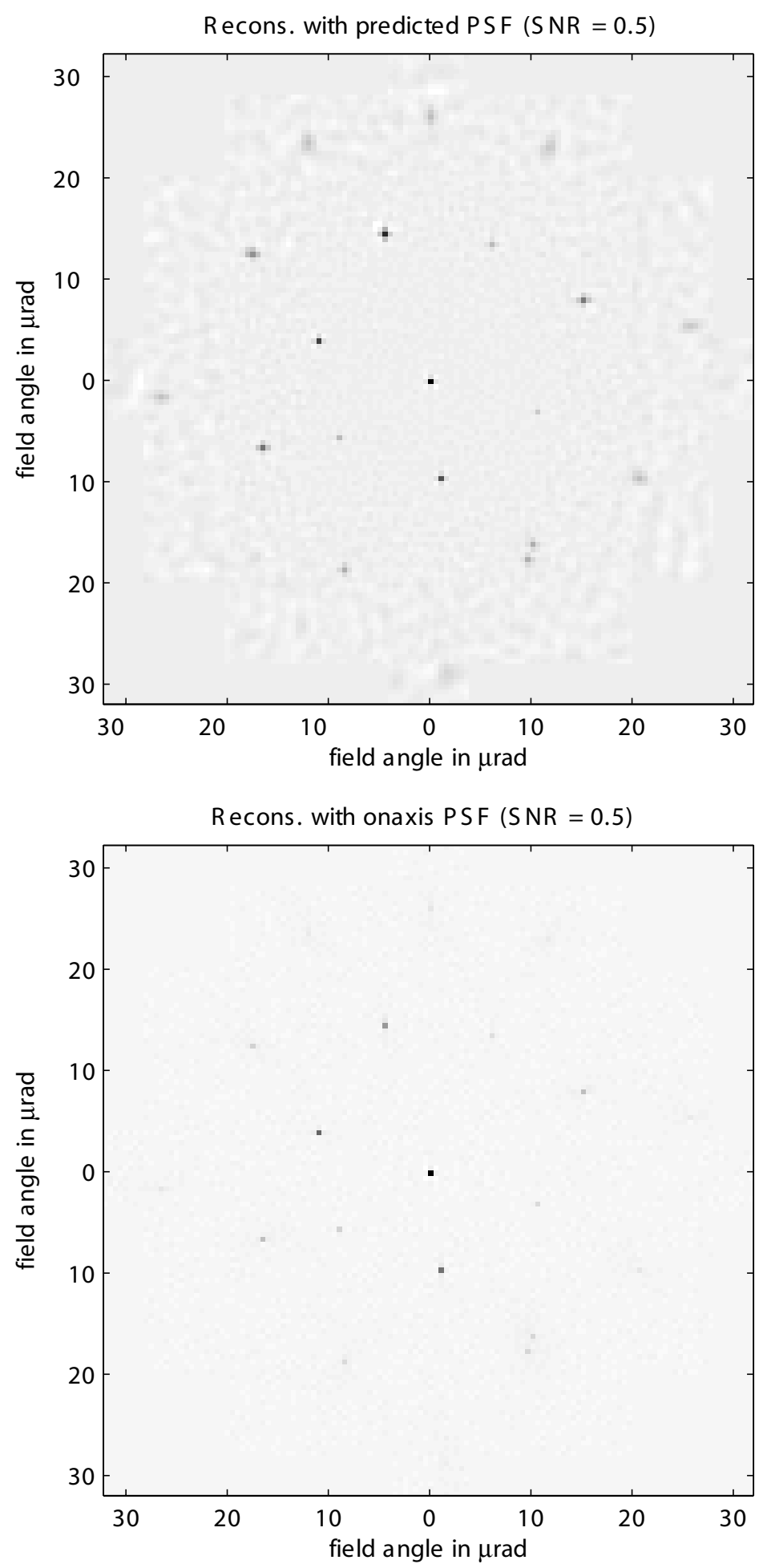

Figure 2.18: Reconstructed images using the Tikhonov regularization with the predicted $P S F$ and the on-axis PSF for $S N R=0.5$ and $r_{0}=10 \mathrm{~cm}$. 

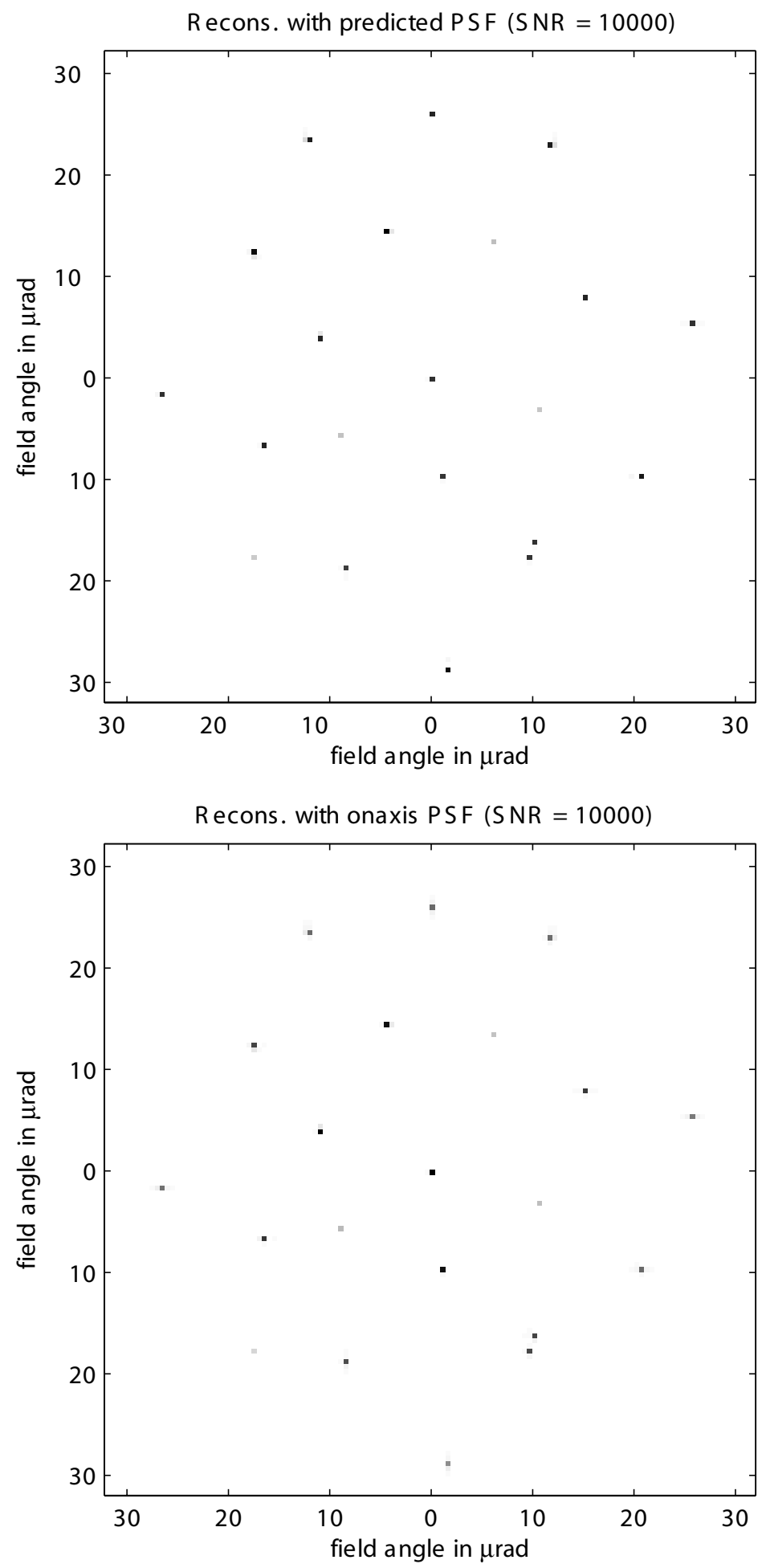

Figure 2.19: Reconstructed images using the EM algorithm with the predicted PSF and the on-axis PSF for $S N R=10000$ and $r_{0}=25 \mathrm{~cm}$. 

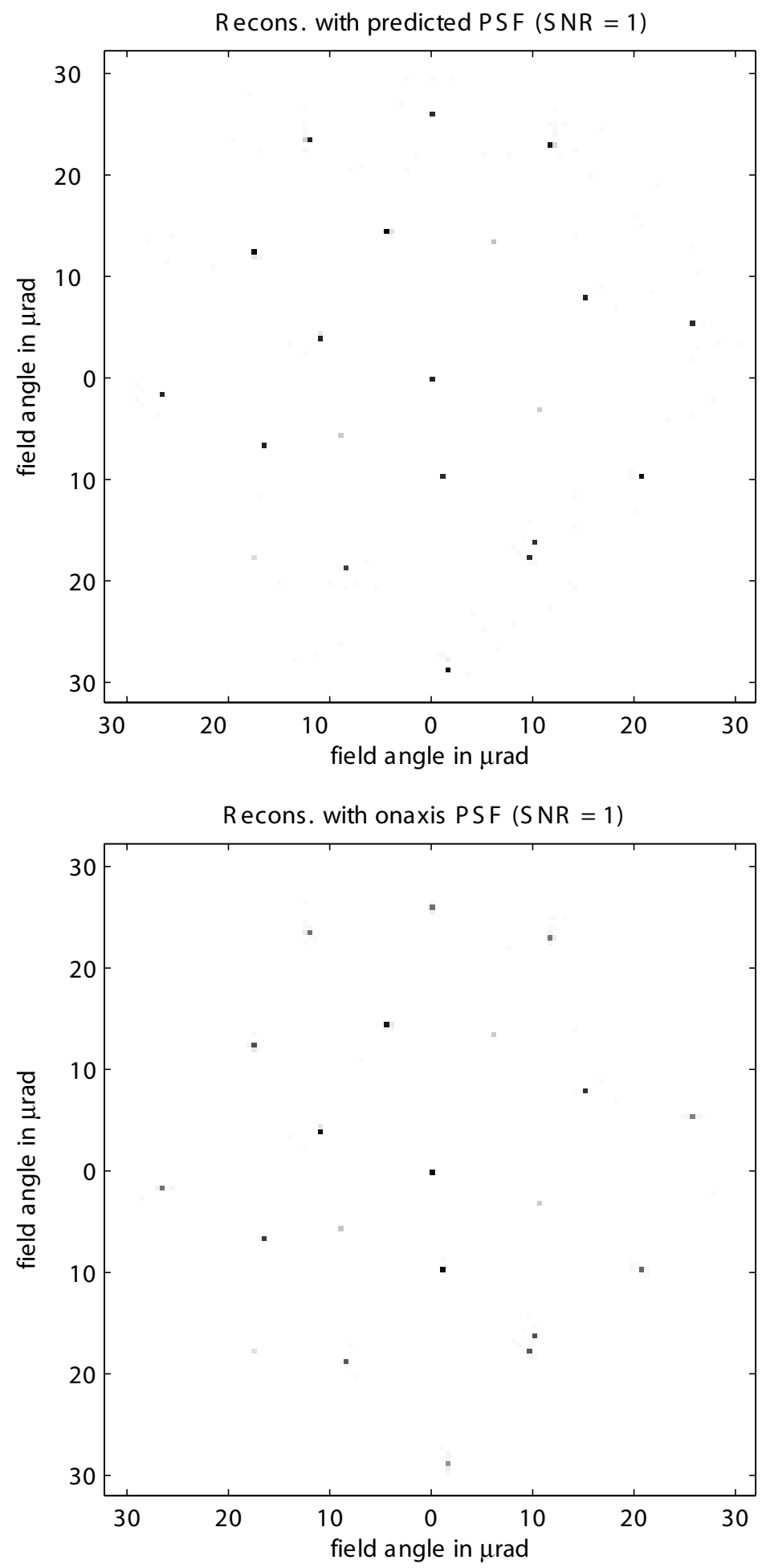

Figure 2.20: Reconstructed images using the EM algorithm with the predicted PSF and the on-axis $P S F$ for $S N R=1$ and $r_{0}=25 \mathrm{~cm}$. 

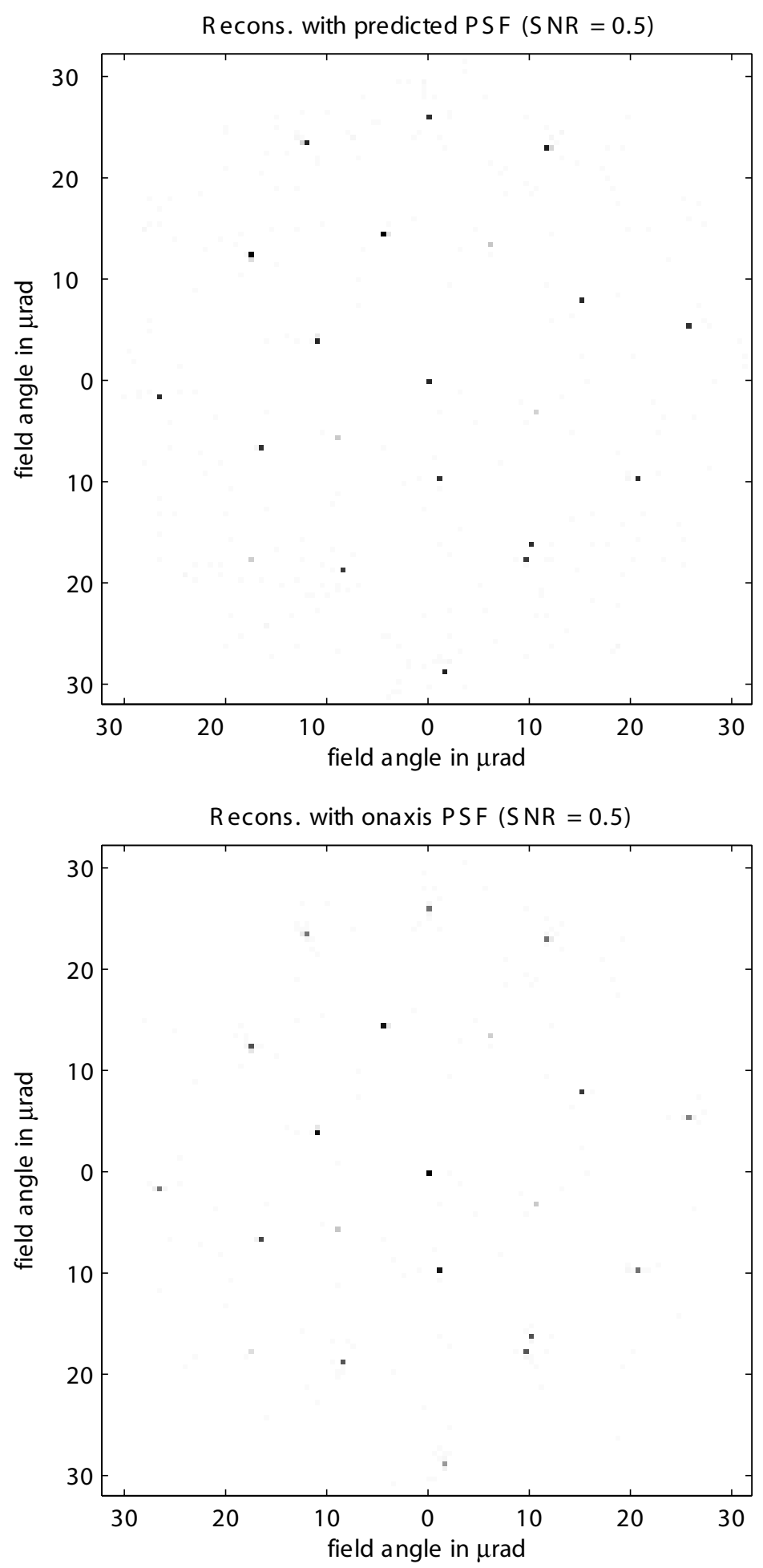

Figure 2.21: Reconstructed images using the EM algorithm with the predicted PSF and the on-axis PSF for $S N R=0.5$ and $r_{0}=25 \mathrm{~cm}$. 

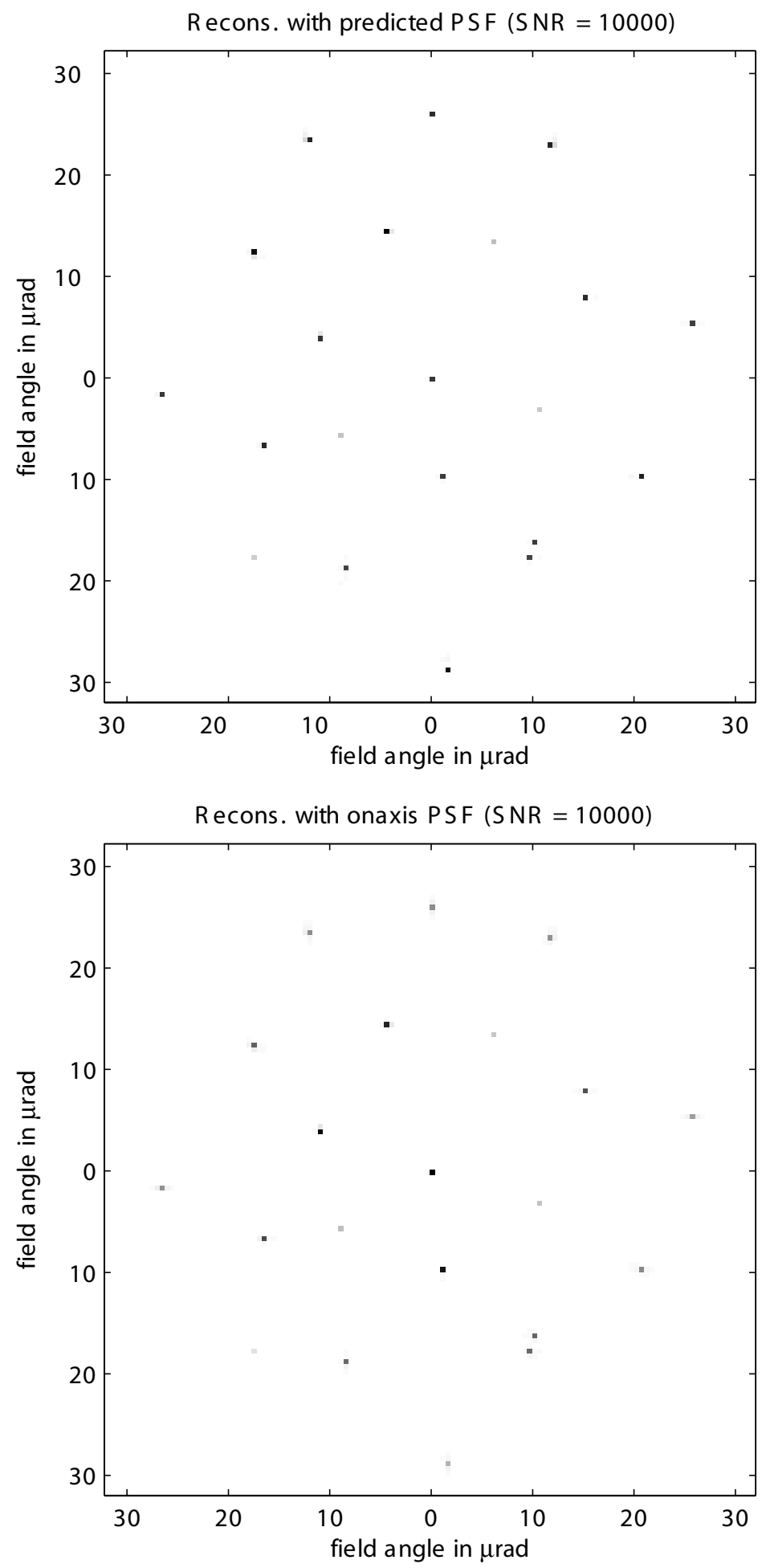

Figure 2.22: Reconstructed images using the EM algorithm with the predicted PSF and the on-axis PSF for $S N R=10000$ and $r_{0}=20 \mathrm{~cm}$. 

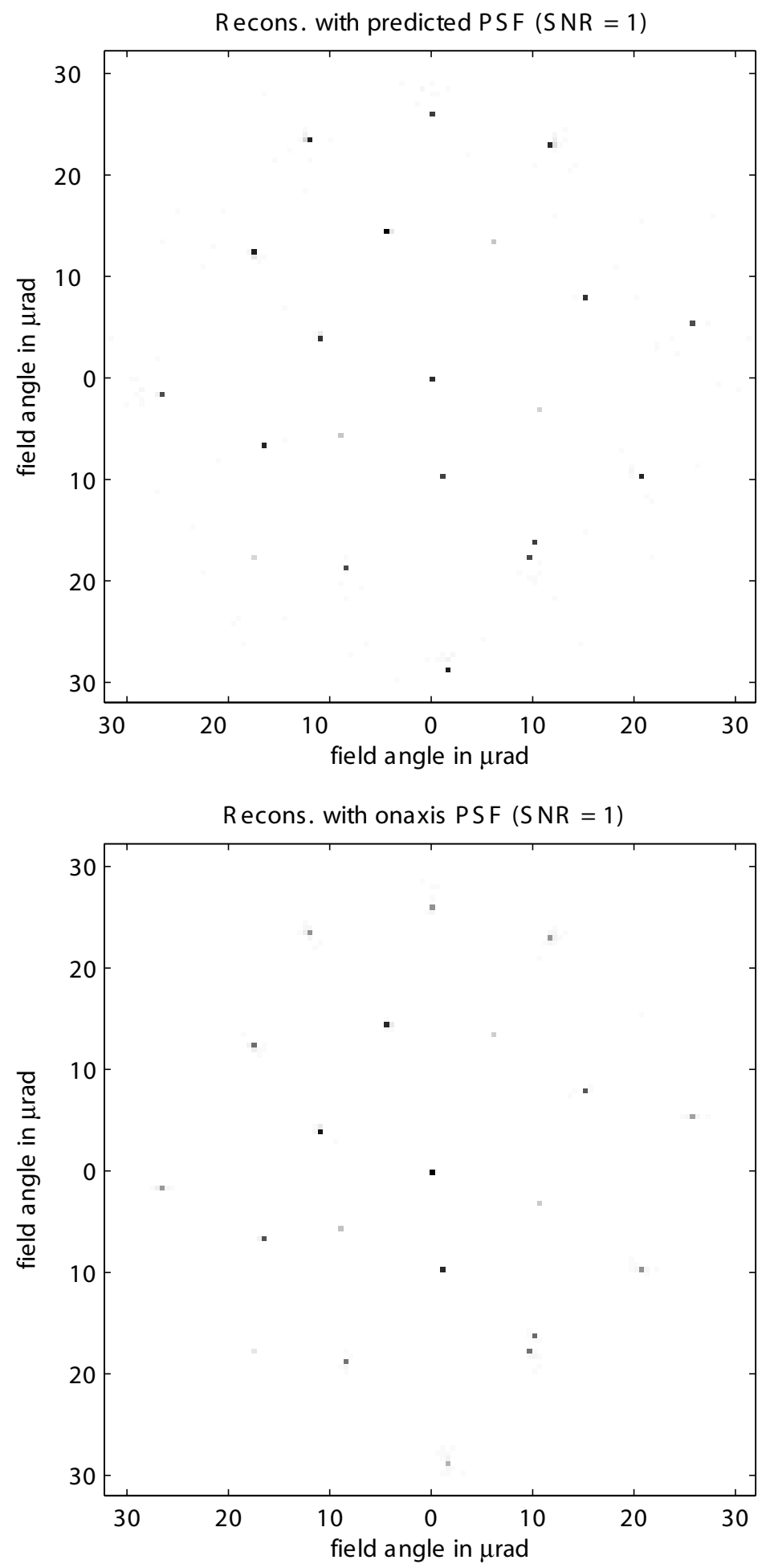

Figure 2.23: Reconstructed images using the EM algorithm with the predicted PSF and the on-axis $P S F$ for $S N R=1$ and $r_{0}=20 \mathrm{~cm}$. 

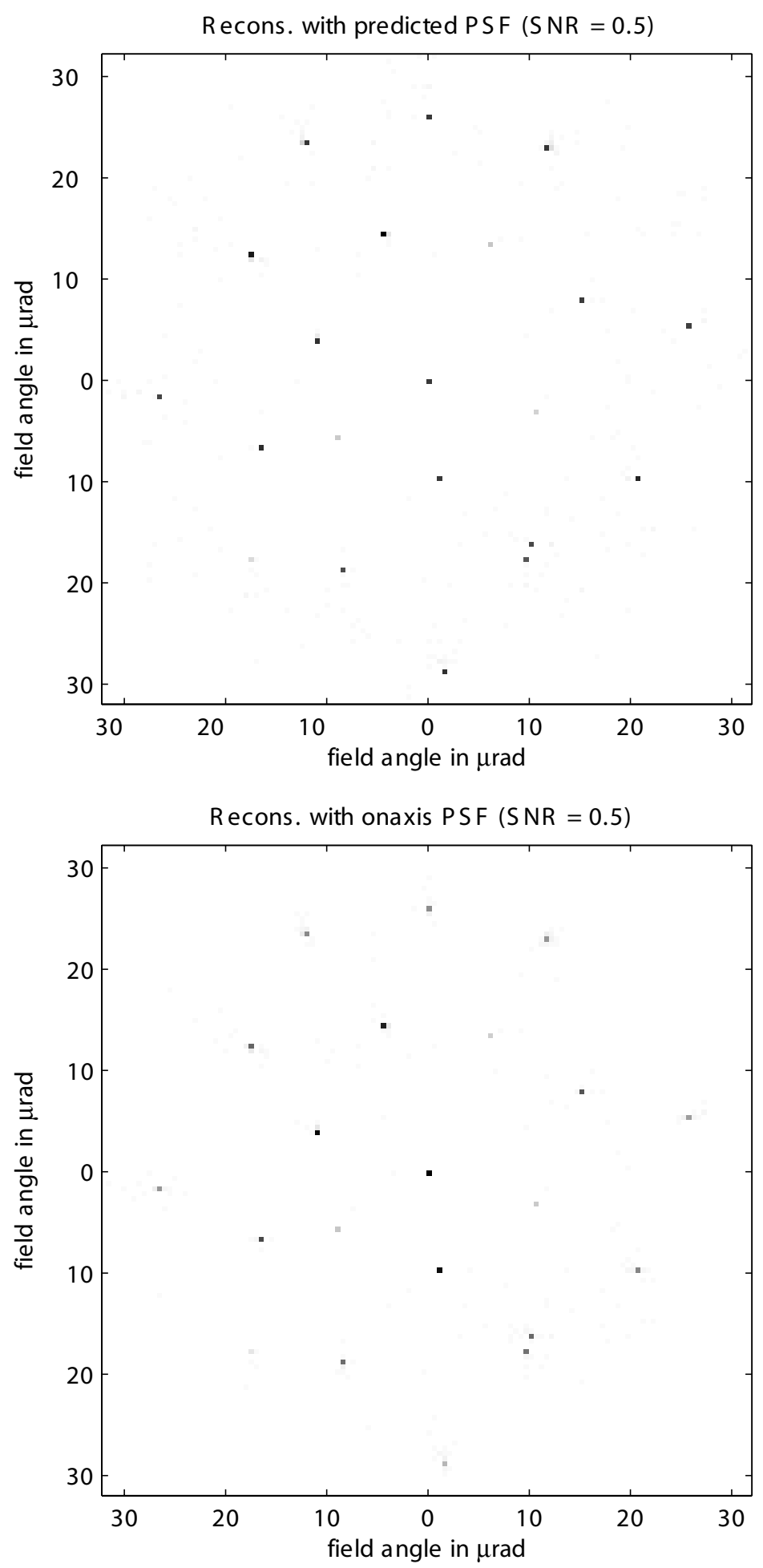

Figure 2.24: Reconstructed images using the EM algorithm with the predicted PSF and the on-axis PSF for $S N R=0.5$ and $r_{0}=20 \mathrm{~cm}$. 

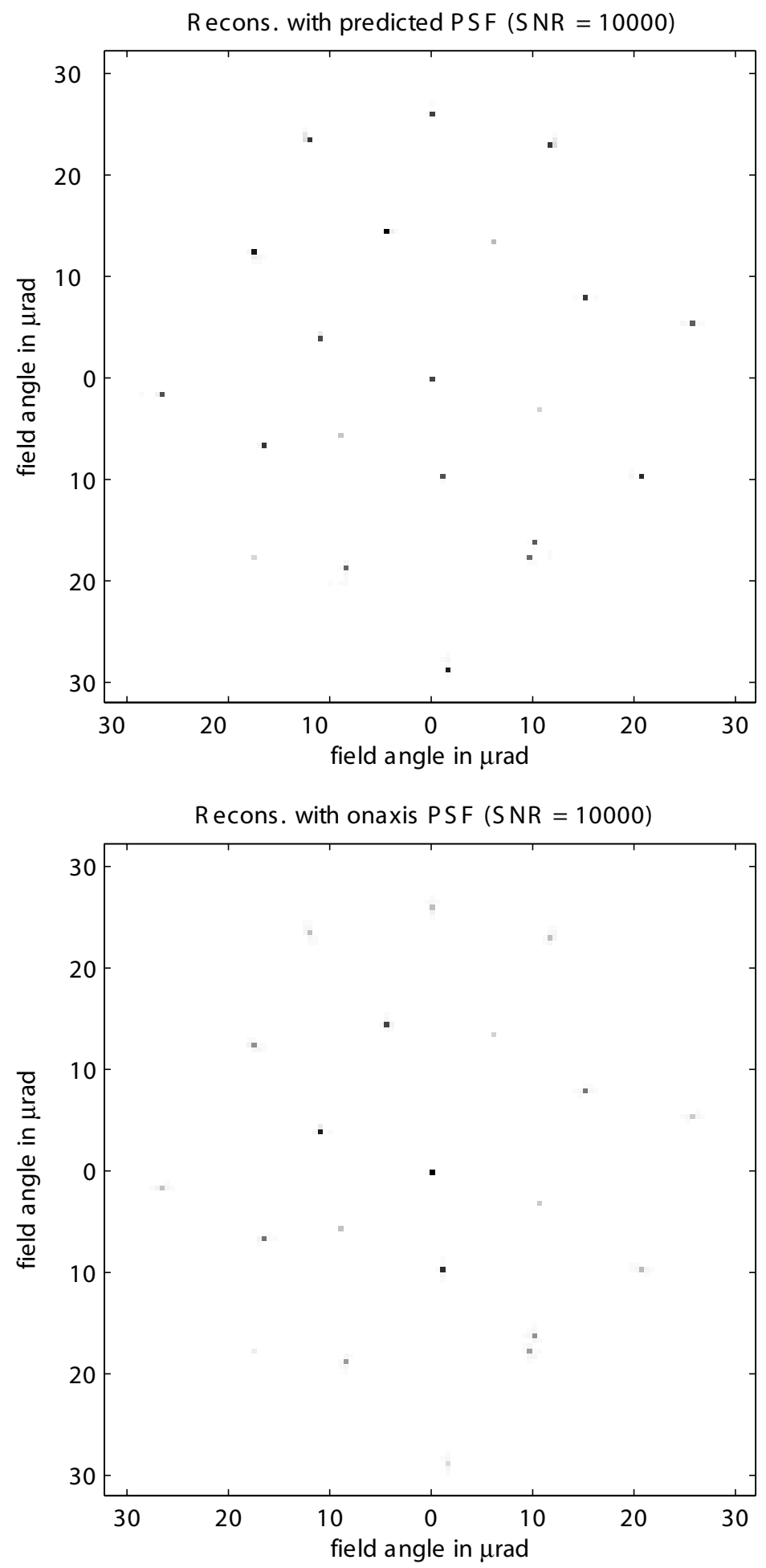

Figure 2.25: Reconstructed images using the EM algorithm with the predicted PSF and the on-axis PSF for $S N R=10000$ and $r_{0}=15 \mathrm{~cm}$. 

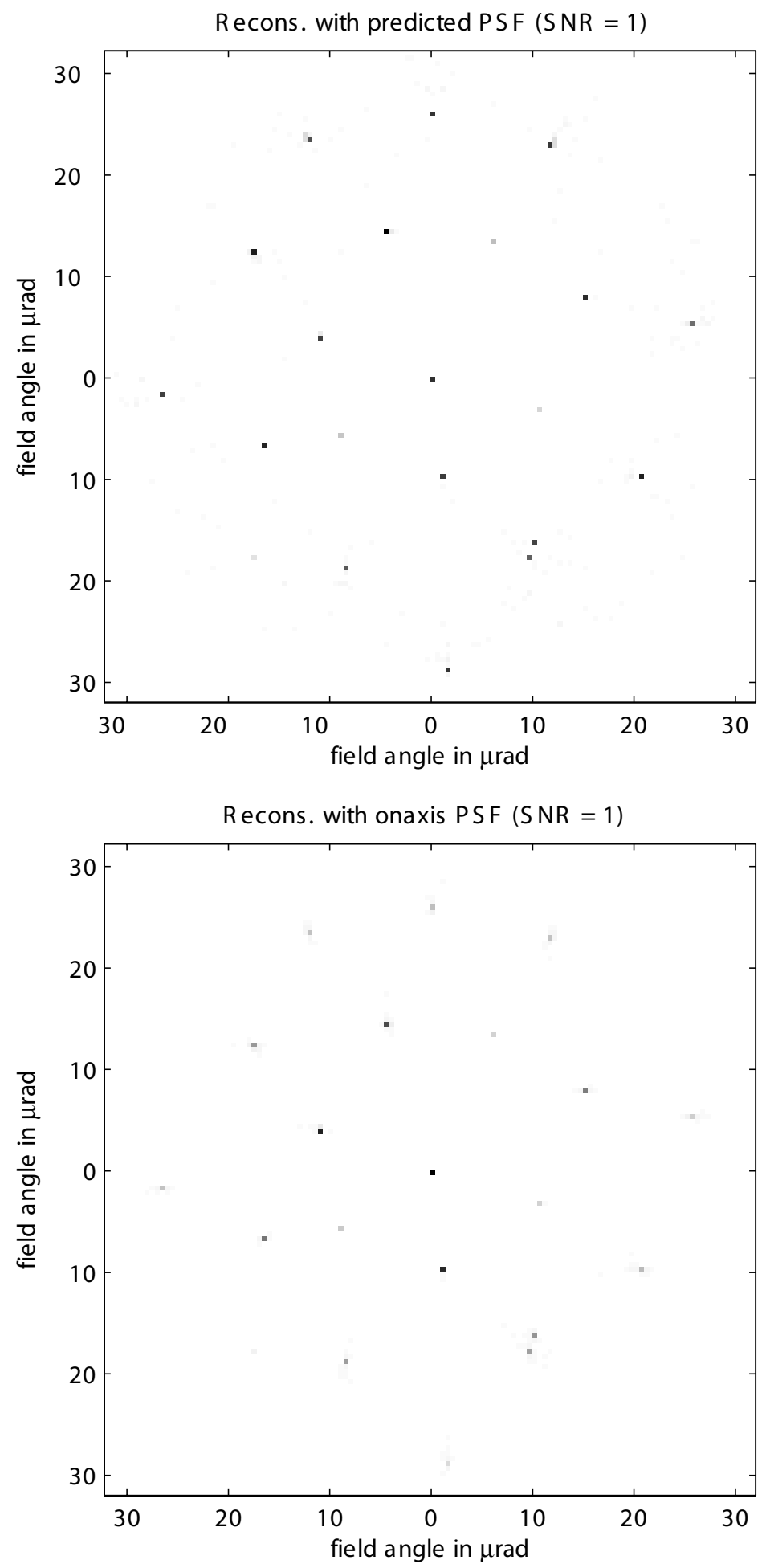

Figure 2.26: Reconstructed images using the EM algorithm with the predicted PSF and the on-axis PSF for $S N R=1$ and $r_{0}=15 \mathrm{~cm}$. 

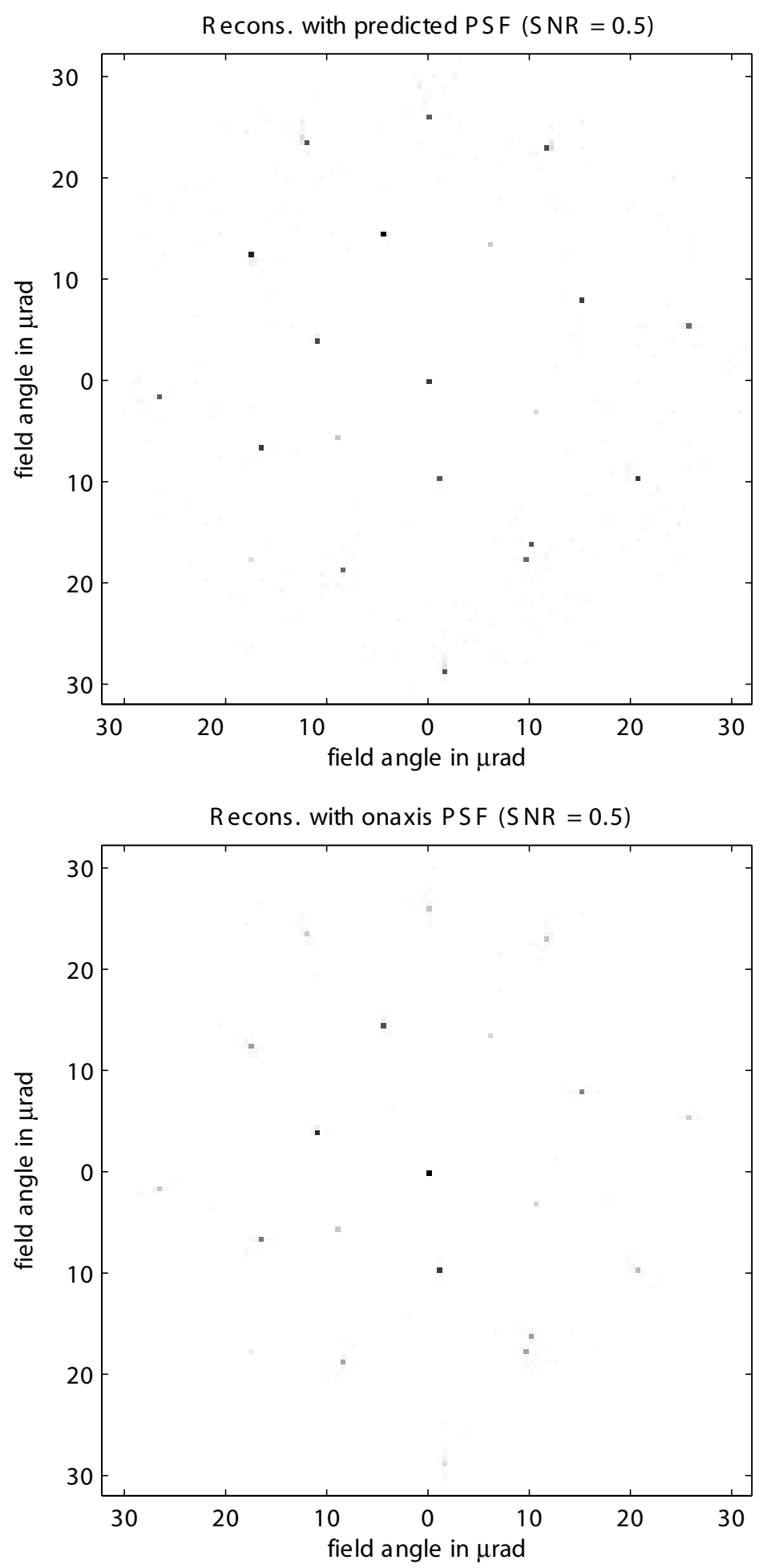

Figure 2.27: Reconstructed images using the EM algorithm with the predicted PSF and the on-axis PSF for $S N R=0.5$ and $r_{0}=15 \mathrm{~cm}$. 

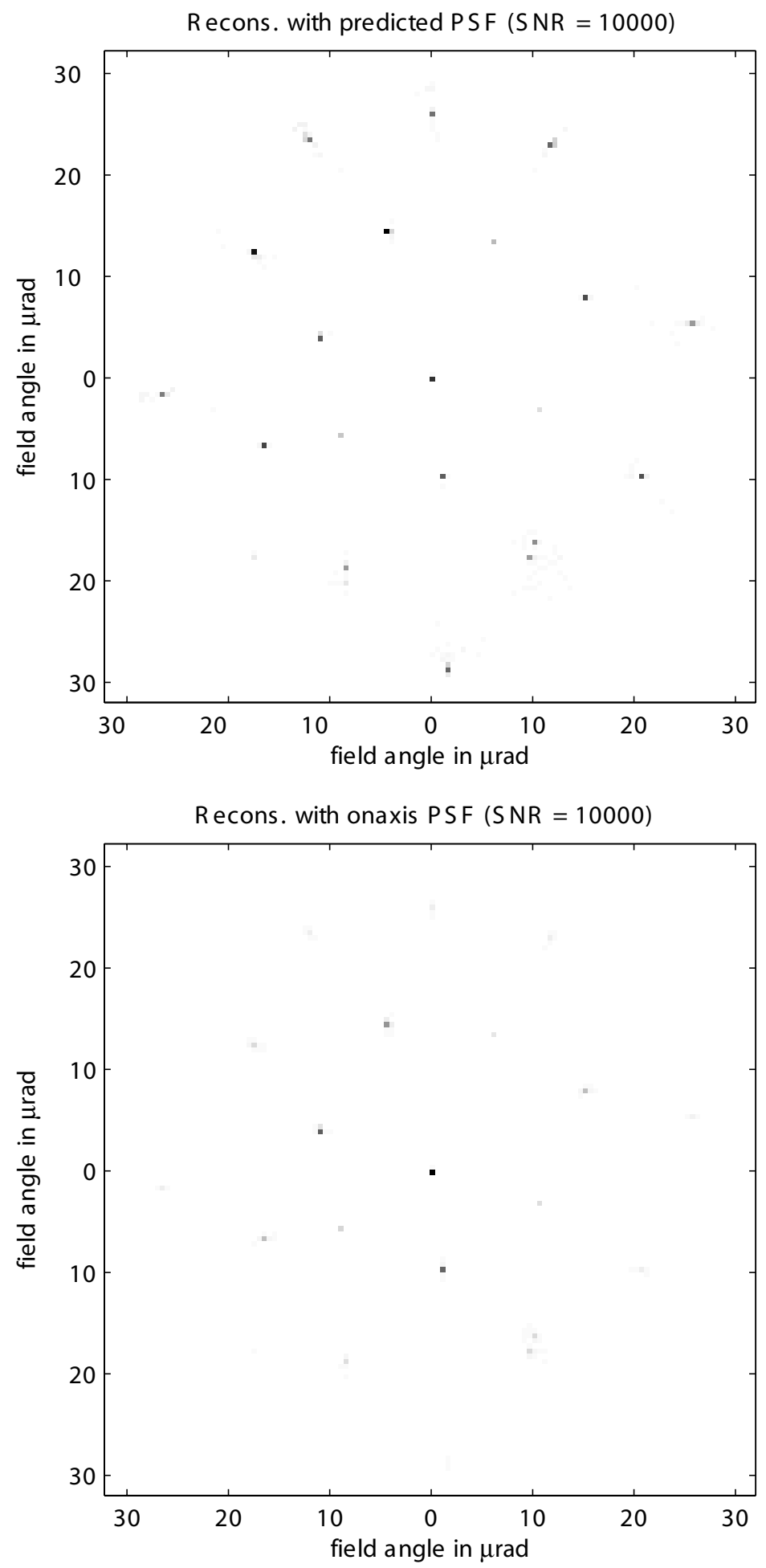

Figure 2.28: Reconstructed images using the EM algorithm with the predicted PSF and the on-axis PSF for $S N R=10000$ and $r_{0}=10 \mathrm{~cm}$. 

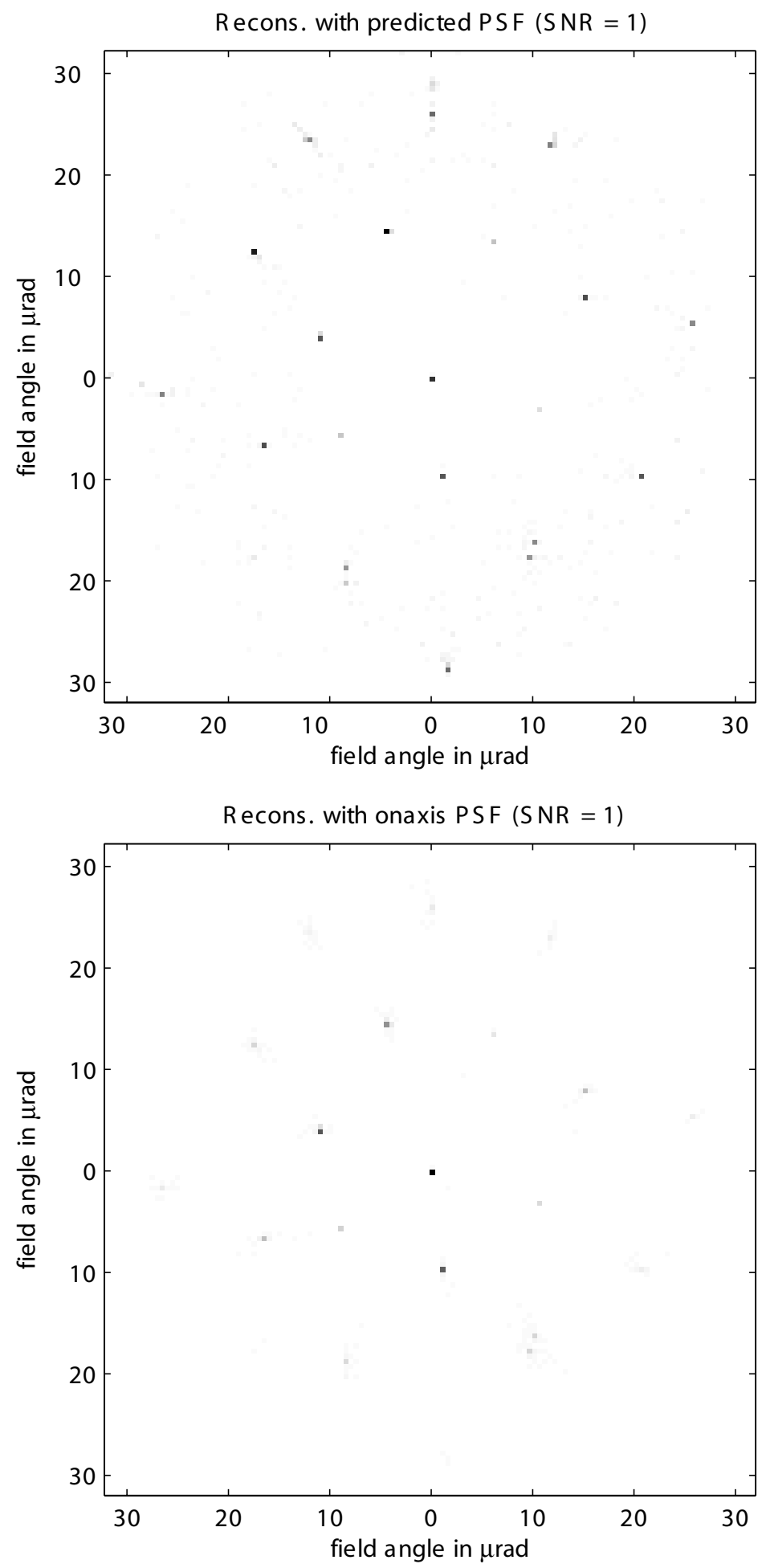

Figure 2.29: Reconstructed images using the EM algorithm with the predicted PSF and the on-axis $P S F$ for $S N R=1$ and $r_{0}=10 \mathrm{~cm}$. 

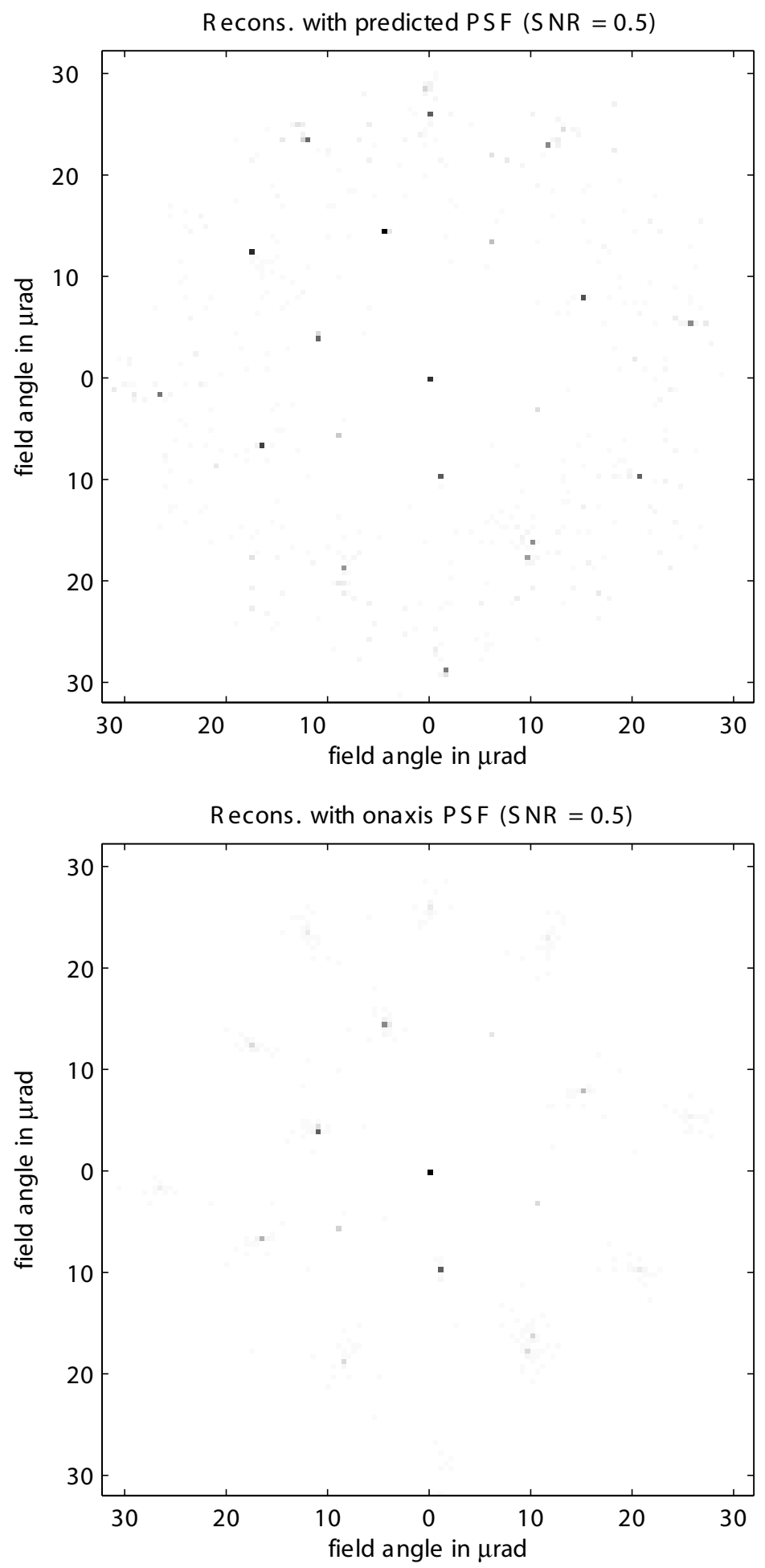

Figure 2.30: Reconstructed images using the EM algorithm with the predicted PSF and the on-axis PSF for $S N R=0.5$ and $r_{0}=10 \mathrm{~cm}$. 


\section{REFERENCES}

[1] European Southern Observatory. www.eso.org.

[2] M. C. Roggemann, B. M. Welsh, and R. Q. Fugate. Improving the resolution of ground-based telescopes. Reviews of Modern Physics, 69:437-505, 1997.

[3] Center for adaptive optics. An NSF science and technology center. http://cfao. ucolick.org.

[4] David L. Fried. Topical issue on adaptive optics. J. Opt. Soc. Am., 67:269-422, 1977.

[5] K. Tyson. Principles of Adaptive Optics. Academic Press, 2nd edition, 1997.

[6] J. P. Gaffard and Corinne Boyer. Adaptive optics for optimization of image resolution. Applied Optics, 26:3772-3777, 1987.

[7] Peter Ninenson and Richard Barakat. Partial atmospheric correction with adaptive optics. J. Opt. Soc. Am., 4:2249-2253, 1987.

[8] Robert Smithson and Michal L. Peri. Partial correction of astronomical images with active mirrors. J. Opt. Soc. Am. A, 6:92-97, 1989.

[9] R. R. Parenti and R. J. Sasiella. Laser-guid-star systems for astronomical applications. J. Opt. Soc. Am., 11:288-309, 1994.

[10] David L. Fried. Anisoplanatism in adaptive optics. J. Opt. Soc. Am., 72(1):52-61, January 1982.

[11] Byron M. Welsh and Chester S. Gardner. Effects of turbulence-induced anisoplanatism on the imaging performance of adaptive-astronomical telescopes using laser guide stars. J. Opt. Soc. Am. A, 8(1):69-80, January 1991.

[12] George C. Valley. Isoplanatic degradation of tilt correction and short-term imaging systems. Applied Optics, 19:574-577, 1980.

[13] A. Sommerfeld. Optics. In Lectures on Theoretical Physics, volume 4. Academic Press, New York, 1954.

[14] J. W. Goodman. Introduction To Fourier Optics. McGraw-Hill Science, 2nd edition, 1996.

[15] M. C. Roggemann and B. Welsh. Imaging through turbulence. CRC Press, Boca Raton, Florida, 1996. 
[16] D. L. Snyder and A. M. Hammoud. Image recovery from data acquired with a charge-coupled-device. J. Opt. Soc. Am. A, 10:1014-1023, 1993.

[17] D. L. Snyder, C. W. Helstrom, A. D. Lanterman, M. Faisal, and R. L. White. Compensation for readout noise in ccd images. J. Opt. Soc. Am. A, 12:272-283, 1995.

[18] Isaak Newton. Opticks. Dover, New York, 4th edition, 1979.

[19] H. W. Babcock. The possibility of compensating astronomical seeing. Publ. Astron. Soc. Pac., 165:229, 1953.

[20] A. N. Kolmogorov. The local structure of turbulence in imcompressible viscous fluids for very large reynolds'numbers. In Turbulence, Classic Papers on Statistical Theory, pages 151-155. Wiley-Interscience, New York, s. k. friedlander and 1. topper edition, 1961.

[21] F. Roddier. The effects of atmospheric turbulence inoptical astronomy. In Progress in Optics, volume XIX. North-Holland, New York, e. wolf edition, 1981.

[22] A. N. Kolmogorov. A rafinement of previous hypotheses concerning the local structure of turbulence in a viscous inhomogeneous fluid at high reynolds number. J. Fluid Mech., 13:82-85, 1962.

[23] A. Ishimaru. Wave Propagation and Scattering in Random Media, volume 2. Academic Press, New York, 1978.

[24] Hufnagel R.E. Technical digest series. J. Opt. Soc. Am., 1974.

[25] D. P. Greenwood. Bandwidth specifications for adaptive optics systems. J. Opt. Soc. Am., 67:390-392, 1977.

[26] J. W. Goodman. Statistical Optics. John Wiley and Sons, 1985.

[27] Michael Bass, E. W. van Stryland, D. R. Williams, and W. L. Wolfe. Handbook of Optics. McGraw-Hill, New York, 2 edition, 1995.

[28] R. F. Lutomirski and H. T. Yura. Wave structure function and mutual coherence function of an optical wave in a turbulent atmosphere. J. Opt. Soc. Am., 61:482487, 1971.

[29] J. M. Martin and S. M. Flatte. Simulation of point-source scintillation through three-dimentional random media. J. Opt. Soc. Am. A, 7:838-847, 1990. 
[30] W. A. Coles, J. P. Filice, R. G. Frehlich, and M. Yadlowsky. Simulation of wave propagation in three-dimentional random media. Applied Optics, 34:2089-2101, 1995.

[31] S. E. Troxel, B. M. Welsch, and M. C. Roggemann. Off-axis optical transfer function calculations in an adaptive-optics system by means of a diffraction calculation for weak index fluctuations. J. Opt. Soc. Am. A, 11:2100-2111, 1994.

[32] J. M. Martin and S. M. Flatte. Intensity images and statistics from numerical simulation of wave propagation in 3-d random media. Applied Optics, 27:2111$2126,1988$.

[33] A. Labeyrie. Attainment of diffraction-limited resolution on large telescope by fourier analyses speckle pattern in stars images. Astron. Astrophys., 6:85, 1970.

[34] Dustin C. Johnston and Byron M. Welsh. Analysis of multiconjugate adaptive optics. J. Opt. Soc. Am., 11(1):394-408, January 1994.

[35] Mette Owner-Petersen and Alexander Goncharov. Multiconjugate adaptive optics for large telescopes: analytical control of the mirror shapes. J. Opt. Soc. Am., 19(3):537-548, March 2002.

[36] G. A. Tyler and D. L. Fried. Image position error associated with a quadrant detector. J. Opt. Soc. Am., pages 804-808, 1982.

[37] B. M. Welsh, B. L. Ellerbroek, M. C. Roggemann, and T. L. Pinnington. Fundamental performance limitations and comparison of the hartmann and shearing interferometer wavefront sensors. Applied Optics, 34:4186-4195, 1995.

[38] H. W. Babcock, B. H. Rule, and J. S. Fassero. An improved automatic guider. Publ. Astron. Soc. Pac., 68(402), 1956.

[39] Scot S. Olivier and Donald T. Gavel. Tip-tilt compensation for astronomical imaging. J. Opt. Soc. Am. A, 11:368, 1994.

[40] G. A. Tyler. Bandwidth considerations for tracking through turbulence. J. Opt. Soc. Am. A, 11:358-367, 1994.

[41] E. N. Ribak. Deformable mirrors. In Adaptive Optics for Astronomy, volume C423 of NATO Advanced Study Institute Series, pages 149-161. Kluwer Academic Publishers, d. alloin and j.-m. mariotti edition, 1994.

[42] D. S. Acton and R. C. Smithson. Solar imaging with a segmented adaptive mirror. Applied Optics, 31:3161-3169, 1992. 
[43] M. A. Ealey and J. A. Wellman. Deformable mirrors: design fundamentals, key performance specifications, and parametric trades. Proc. SPIE on Active and Adaptive Optical Components, 1543:36-51, 1991.

[44] B. Hulburd and D. Sandler. Segmented mirrors for atmospheric compensation. Opt. Eng., 29:1186-1190, 1990.

[45] R. H. Hudgin. Wavefront compensation error due to finite corrector-element size. J. Opt. Soc. Am., 67:393-395, 1977.

[46] E. P. Wallner. Optimal wave front correction using slope measurements. J. Opt. Soc. Am., 73:1771-1776, 1983.

[47] B. M. Welsch and C. S. Gardner. Performance analysis of adaptive optics systems using laser guide stars and slope sensors. J. Opt. Soc. Am. A, 6:1913-1923, 1989.

[48] J. W. Hardy. Adaptive optics: a new technology for the control of light. Proc. IEEE, 66:651, 1978.

[49] K. E. Moore and G. N. Lawrence. Zonal model of adaptive model. Applied Optics, 29:4622-4628, 1990.

[50] V. I. Tatarskii. Wave Propagation in a Turbulent Medium. New York: Dover Publications, 1967.

[51] J. L. Melsa and D. L. Cohn. Decision and Estimation Theory. New York: McGraw-Hill Book company, 1978.

[52] S. M. Kay. Fundamentals of statistical signal processing: Estimation theory. Prentice Hall, Englewood Cliffs, NJ, 1993.

[53] R. K. Tyson. Adaptive optics system performance approximations for atmospheric turbulence correction. Opt. Eng., 29:1165-1173, 1990.

[54] M. C. Roggemann. Optical performance of fully and partially compensated adaptive optics systems using least-squares and minimum variance phase reconstruction. Comp. and Elec. Eng., 18:451-466, 1989.

[55] M. Born and E. Wolf. Principles of Optics. Pergamon, Oxford, 5th edition, 1975.

[56] K. Binder and D. W. Heermann. Monte Carlo simulation in statistical physics. Springer, 3rd edition, 1997.

[57] WaveTrain. Mza associates corporation, www.mza.com.

[58] Matlab. The mathworks, inc., www.mathworks.com. 
[59] AOTools. the optical sciences company, www.tosc.com.

[60] A. Papoulis. Probability, random variables, and stochastic processes. McGrawHill, New York, 3rd edition, 1991.

[61] G. Strang. Linear algebra and its applications. Academic Press, New York, 2nd edition, 1980.

[62] W. Press, B. Flannery, S. Teukolsky, and W. Vetterling. Numerical recipes - The art of scientific computing. Cambridge University Press, Cambridge, UK, 1986.

[63] A. J. Lambert and D. Fraser. Linear system approach to simulation of optical diffraction. Applied Optics, 37:7933-7939, 1998.

[64] M. C. Roggemann and D. J. Lee. Two-deformable-mirror concept for correcting scintillation effects in laser beam projection through the turbulent atmosphere. Applied Optics, 37:4577-4585, 1998.

[65] R. Kizito. Image sharpness metric-based deformable mirror control for beam projection system. PhD thesis, Michigan Technological University, 1400 Townsend Dr., Houghton, 49931, 2004.

[66] J. Christou, E. Steinbring, S. Faber, D. Gavel, J. Patience, and E. Gates. Anisoplanatism within the isoplanatic patch. Am. Astron. Soc., 34:1257, 2002.

[67] E. Steinbring, S. M. Faber, S. Hinkley, B. A. Macintosh, D. Gavel, E. L. Gates, Julian C. Christou, M. Le Louarn, L. M. Raschke, Scott A. Severson, F. Rigaut, David Crampton, J. P. Lloyd, and James R. Graham. Characterizing the adaptive optics off-axis point-spread function. i. a semiempirical method for use in natural guide star observations. Astron. Soc. of the Pac., 114:1267-1280, 2002.

[68] K. Vogel. Computational methods for inverse problems. SIAM, 2002.

[69] R. C. Gonzales and R. E. Woods. Digital image processing. Addison-Wesley Publisher, 1992.

[70] A. N. Tikhonov, A. V. Goncharsky, V. Stepanov, and A. G. Yagola. Numerical methods for the solution of ill-posed problems. Kluwer Academic Publisher, 1995.

[71] M. Bertero and P. Boccaci. A simple method for the reduction of boundary effects in the richardson-lucy approach to image deconvolution. Astron. Astrophys., 2005.

[72] Curtis R. Vogel and Mary E. Oman. Fast, robust total variation-based reconstruction of noisy, blurred images. IEEE Transactions on Image Processing, 7(6):813824, June 1998. 
[73] M. Bertero, C. De Mol, and G. A. Viano. Restoration of optical objects using regularization. Optics letters, 3(2):51-53, August 1978.

[74] M. Piana and M. Bertero. Regularized deconvolution of multiple images of the same object. J. Opt. Soc. Am. A, 13(7):1516-1523, July 1996.

[75] Martin Hanke. Surveys on solution methods for inverse problems, pages 35-52. D. Colton et al., springer-verlag edition, 2000.

[76] W. H. Richardson. Bayesian-based iterative method of image restoration. J. Opt. Soc. Am., 62(1):55-59, January 1972.

[77] L. B. Lucy. An iteration technique for the rectification of observed distributions. Astron. J., 79:745-754, 1974.

[78] D. L. Phillips. A technique for the numerical solution of certain integral equations of the first kind. J. Assoc. Comp. Mach., 9:84-97, 1962.

[79] A. Dempster, N. Laird, and D. Rubin. Maximum likelihood from incomplete data via the em algorithm. J. of the Royal Stat. Soc., 39(1):1-38, 1977.

[80] A. Abu-Naser, N. P. Galatsanos, and M. N. Wernick. Object recognition based on impulse restoration with use of the expectation-maximization algorithm. J. Opt. Soc. Am. A, 15(9):2327-2340, 1998.

[81] T.J. Holmes. Expectation-maximization restoration of band-limited, truncated point-process intensities with application in microscopy. J. Opt. Soc. Am. A, 6(7):1006-1014, 1989.

[82] D. A. Fish, A. M. Brinicombe, and E. R. Pike. Blind deconvolution by means of the richardsonlucy algorithm. J. Opt. Soc. Am. A, 12(1):58-65, 1995.

[83] Sudhakar Prasad. Statistical-information-based performance criteria for richardsonlucy image deblurring. J. Opt. Soc. Am. A, 19(7):1286-1296, 2002.

[84] Geoffrey McLachlan and Thriyambakam Krishnan. The EM algorithm and extensions. John Wiley and Sons, New York, 1996.

[85] Maui Space Surveillance System (MSSS). www.maui.afmc.af .mil. 